
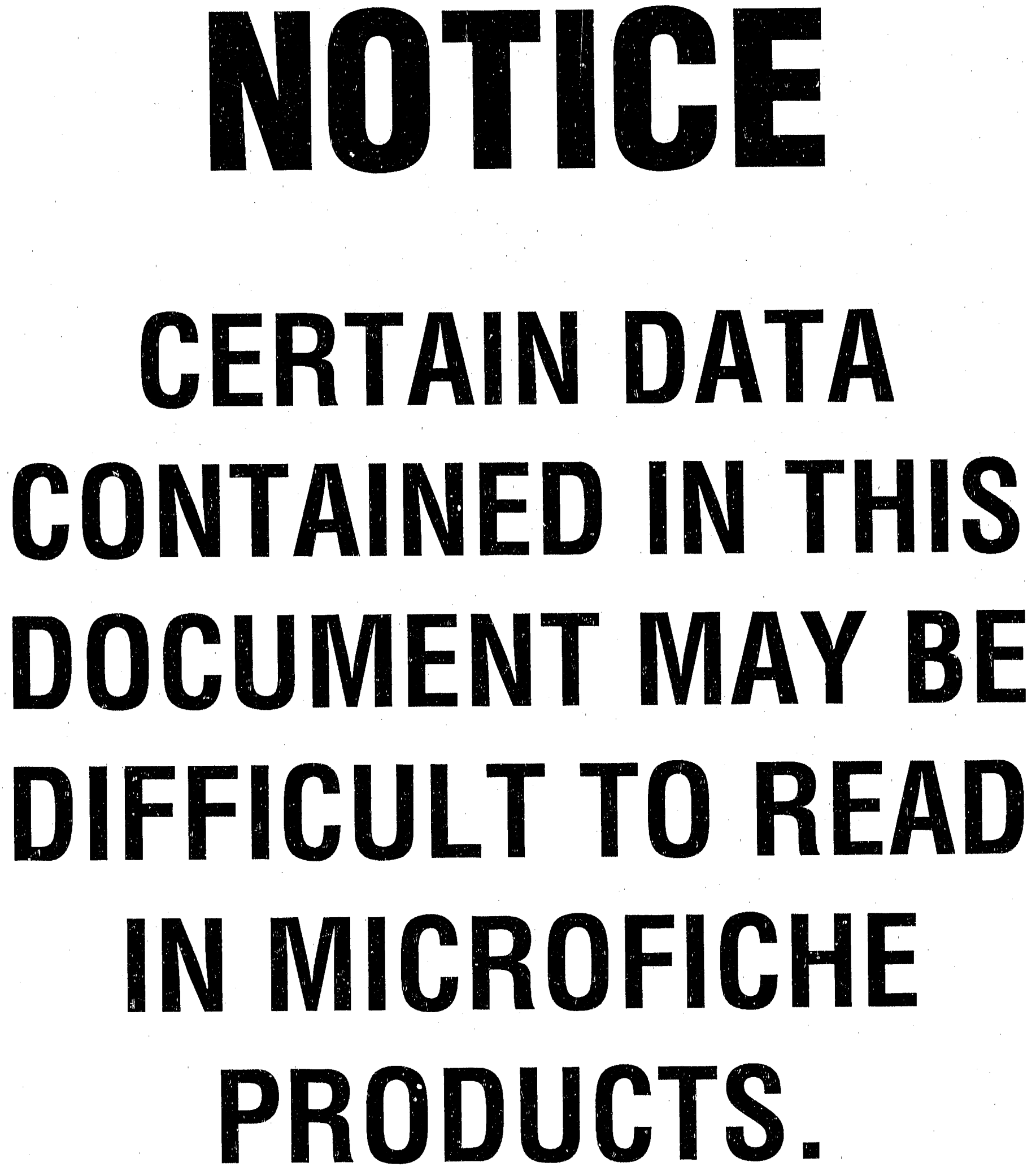

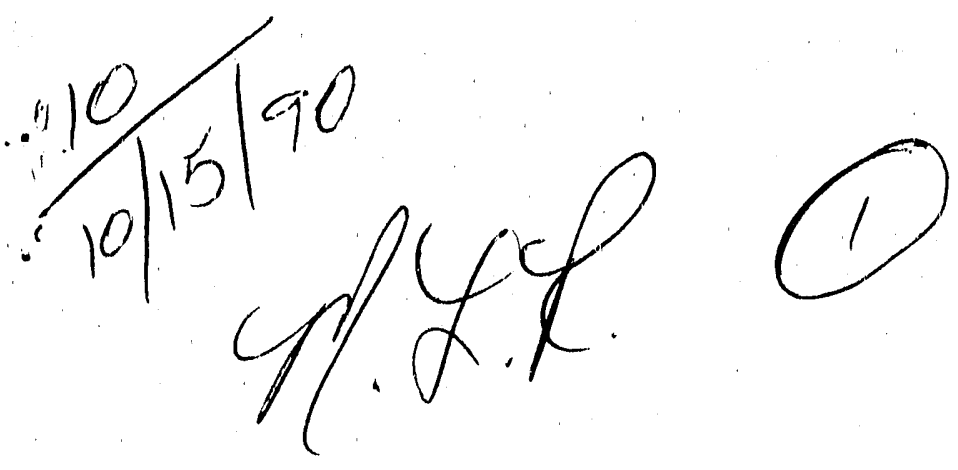

\title{
INGRID by Example A Pictorial Tutorial
}

\author{
Robert Rainsberger
}

November, 1988
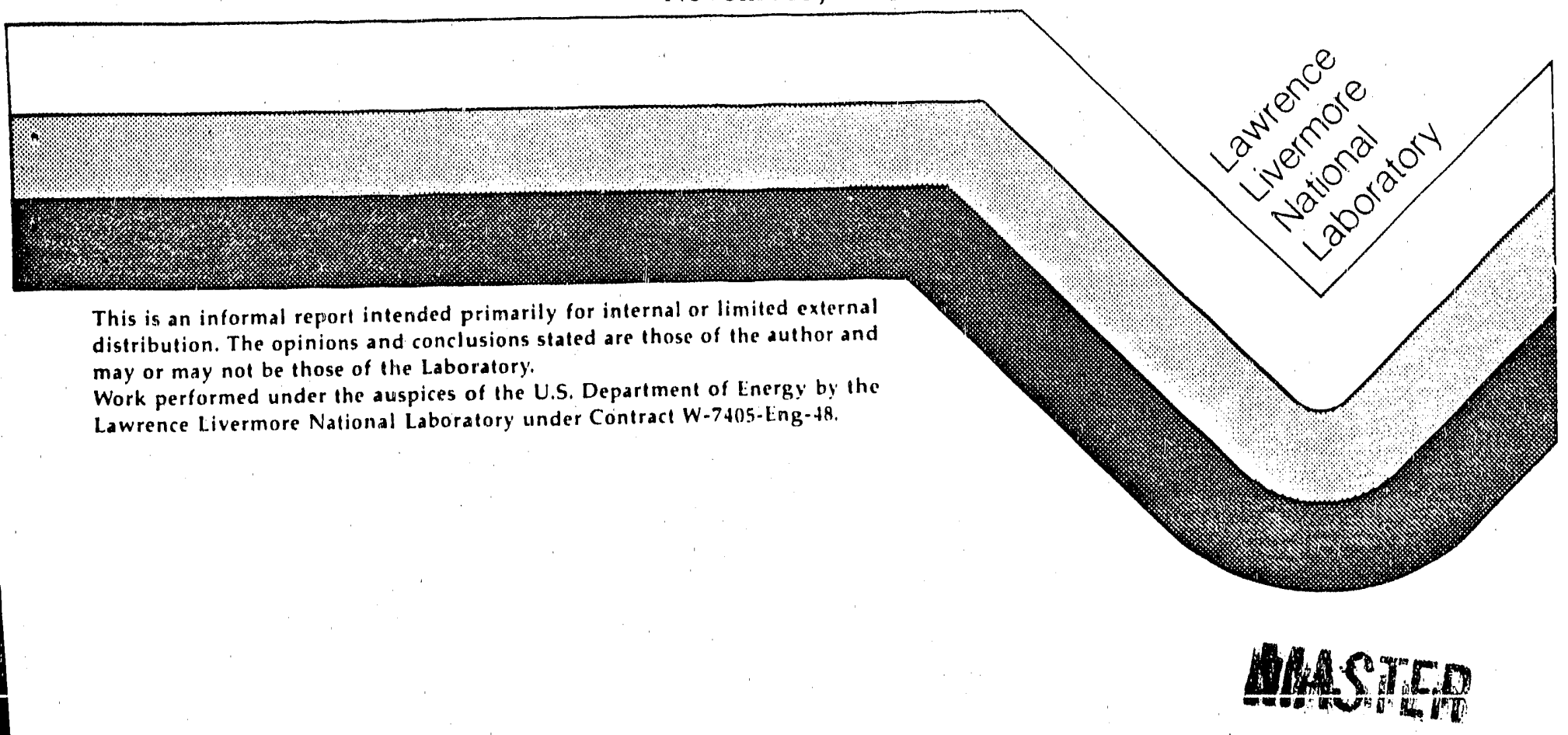


\title{
DISCLAIMER
}

This document was prepared as an account of work oponsored by an agency of the Unitsd States Covernment. Nother the United Stales Government nor the Univeroify of California nor any of their employees, makes any warranty, express or implied, or aseumes any lebal liability or responsibillty for the accuracy, completeneas, or usefulness of any information, apparafus, product, or process disclosed, or represents that its use would not infringe privately owned rights. Reference herein to any opecific commercial products, procets, or eetrice by trade name, trademark, manufactures, of otherwise, does not necessarily conotitute or impiy lis endoreement, recommendation, or favoring by the Uniled States Covernment or the University of California. The view's and opinions of authors expressed herein do not necesurily state or reflect those of the United Staten Covemment of the Lniversity of California, and whall not be used for advertising or product endonement purposes.

\author{
Mnnied in the Uniled Slales of Americe \\ Availathle from \\ Nalional Terhnical Information Survice \\ U.S Department of Commetce \\ 3285 I'on Royal Rosd \\ Spnnglield, VA 22161
}

\section{Papercopy Prices}

A02

$\mathrm{A03}$

A04

A05

A06

A0\%

A08

A09
$001-050$

051-100

101-200

$201-300$

$301-400$

$401-500$

501-600

601 


\section{INGRID BY EXAMPLE A PICTORIAL TUTORIAL*}

The following examples of INGRID input files and results are from various sources including Doug Stillman. In many cases, they are the same as in the examples section of the INGRID manual by Doug Stillman. I have made some modest improvements so that they are all complete input files to be run with the latest version of INGRID. These files have been attached to the front end of the INGRID source code as comments.

I wish to invite all INGRID user's to submit any favorite input files to be included in this tutorial. Such an input file should not be too large, and the object being generated should have a natural appeal to INGRID users. Feel free to make suggestions.

This tutorial will soon replace the examples section of the manual. The entire manual will be replaced a section at a time. I chose to do this section first, since it could give the most benefit in the shortest amount of time. As soon as I make INGRID interactive, I will then rewrite the section on standard parts.

\footnotetext{
"Worked performed under the auspices of the U.S. Department of Energy by the Lawrence Livermore National Laboratory under contract number W-7405-Eng-48.
} 


\section{INGR I D}

Golden Gate Bridge Finite Element Model by Robert Rainsberger Reference: The Golden Gate Bridge Final Report by R. Strauss
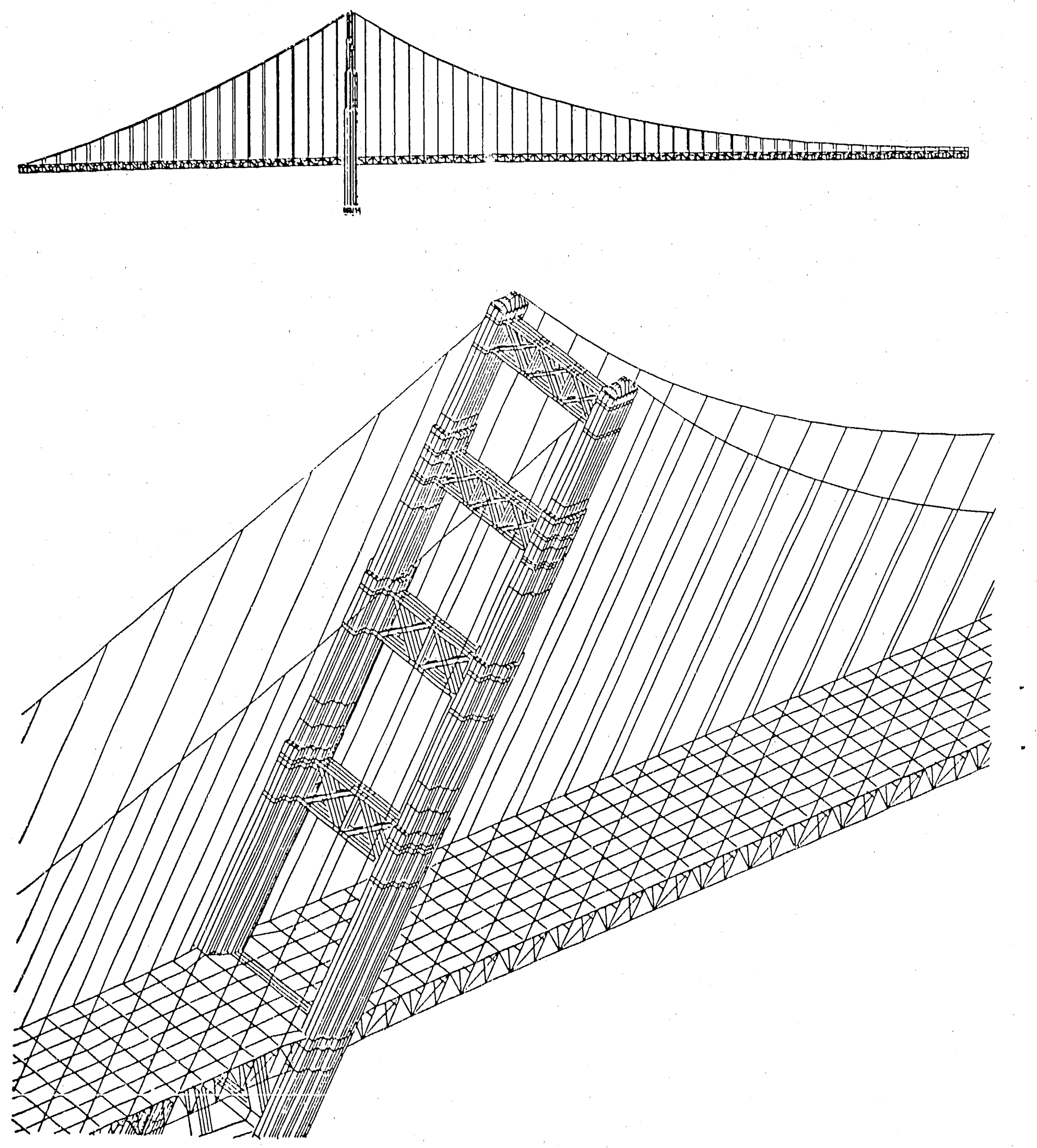


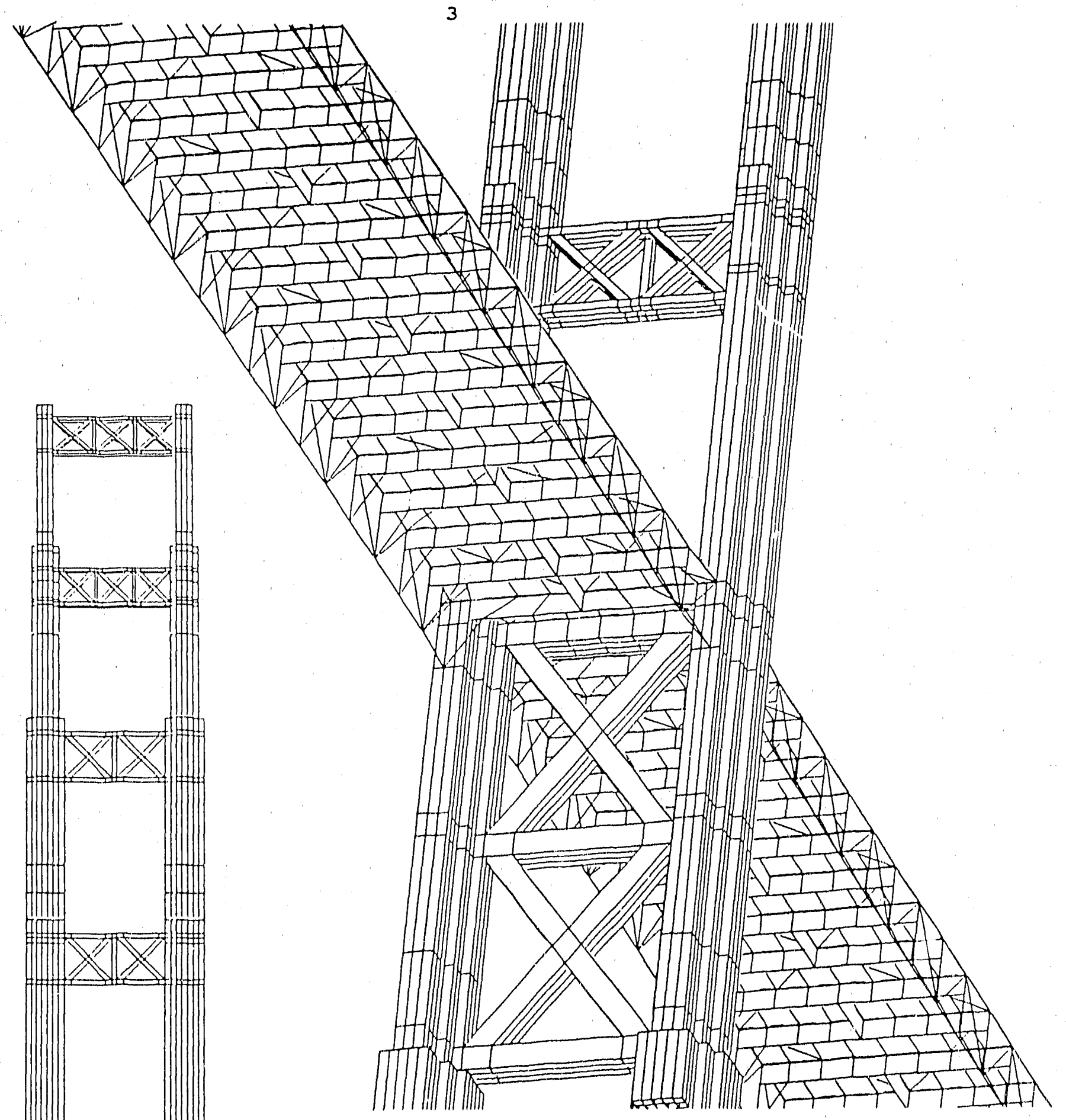


imple cube from a standard part with one element n3d

\section{tart}

$2: 12: 12$;

21212

nd

, nd

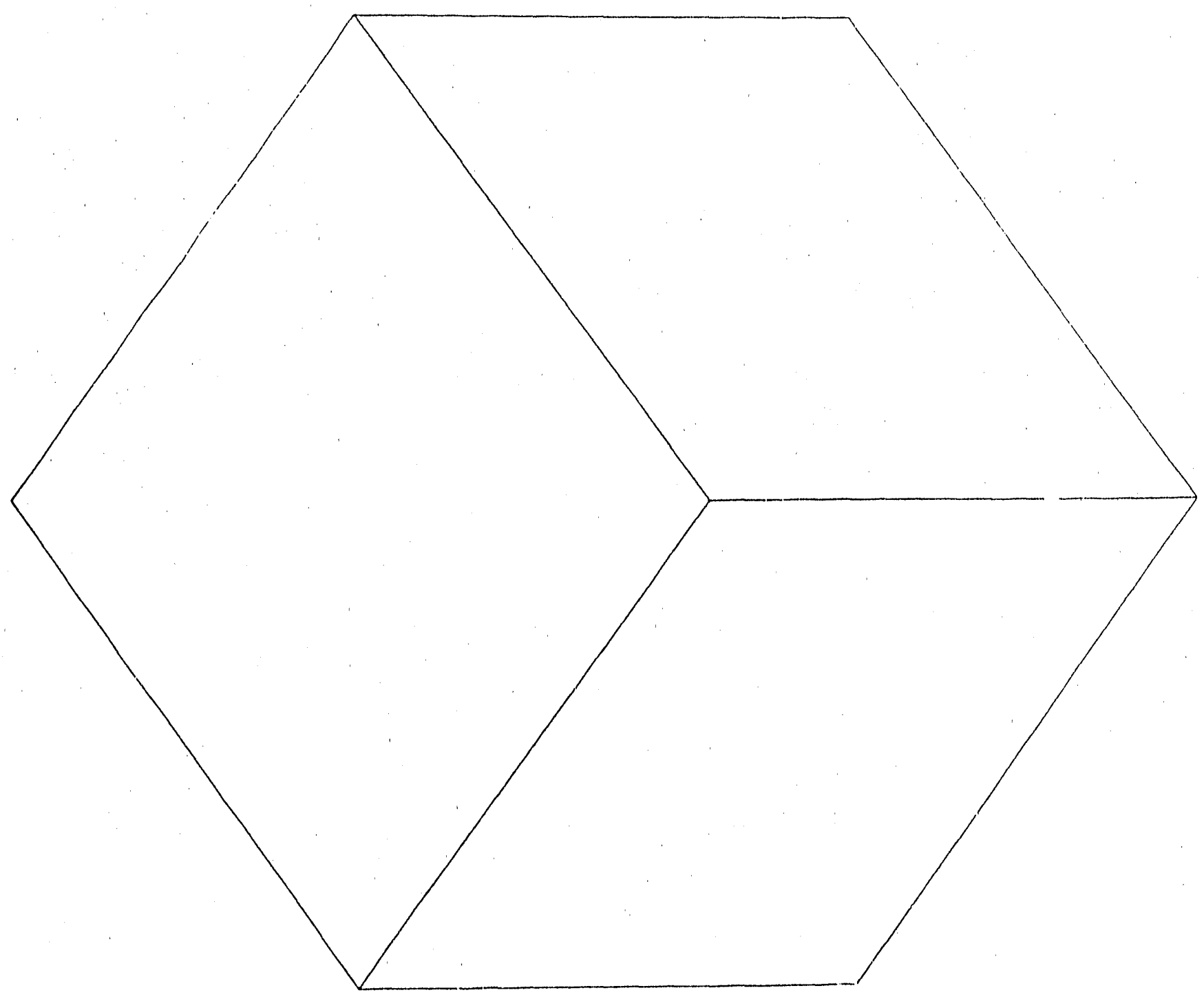


simple cube from a standard part with sections deleted dn $3 d$

start $16 \begin{array}{llllllllllll} & 6 & 16 & 21: 1 & 6 & 11 & 16 & 21: 1 & 6 & 11 & 16 & 21:\end{array}$

$\begin{array}{lllllllllllllllll}1 & 2 & 4 & 8 & 16 & 1 & 2 & 4 & 8 & 16 & 1 & 2 & 4 & 8 & 16\end{array}$

di $12045: 12045: 12045$ :

end end

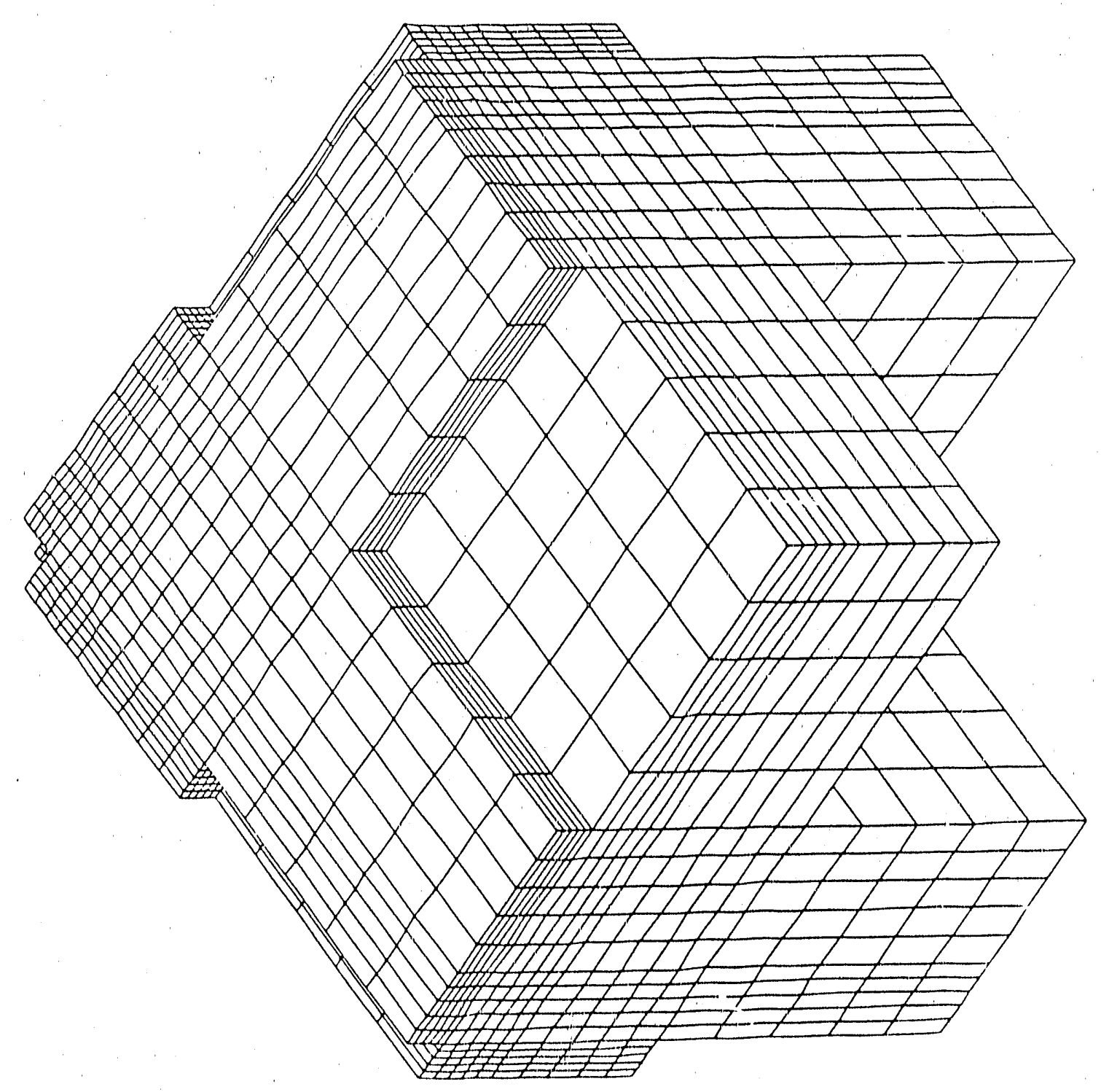




$$
\begin{aligned}
& \text { simple example of a solid cylinder } \\
& \text { dna } 3 d \text {. } \\
& \text { start } \\
& 14: 137: 110: \\
& 120360114 \\
& \text { cyl } 1 \text {. } \\
& \text { end } \\
& \text { end }
\end{aligned}
$$

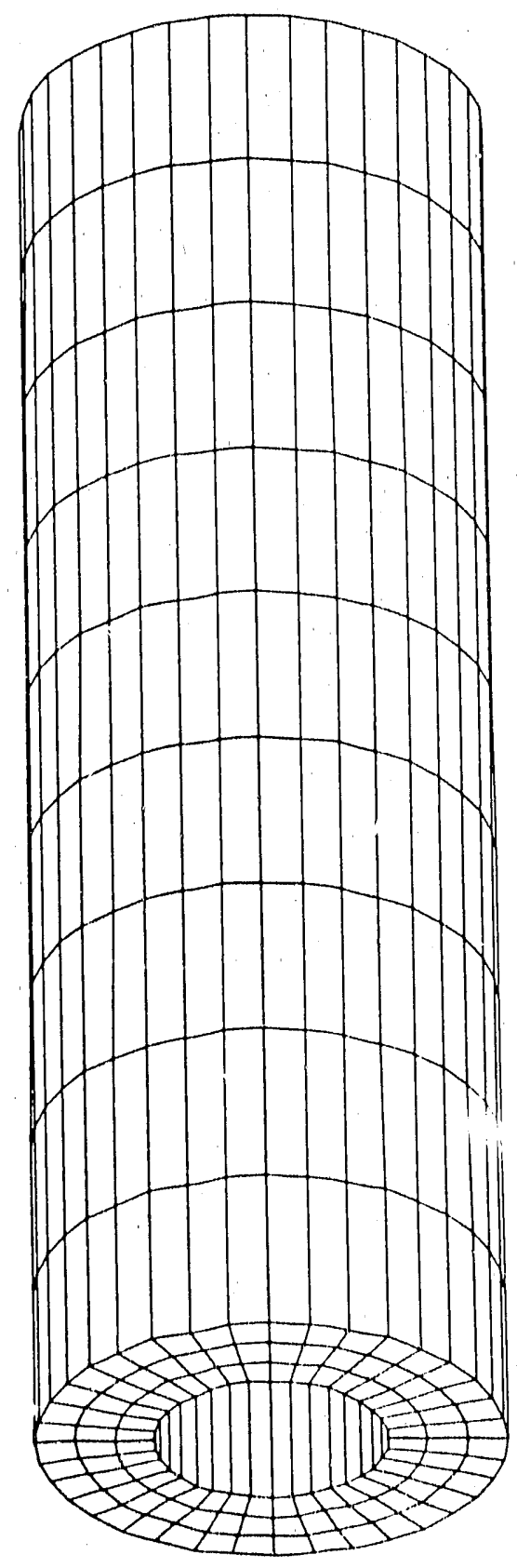


$\therefore \quad$ telescoping cylinders

dn3d

- start

$1234456: 1 \quad 37: 14471013$ 16:

1234560360123456

cyli

d 2005300

d. 3004406

d 4003500

$\begin{array}{lllllll} & 5 & 0 & 2 & 6 & 0 & 6\end{array}$

end

end

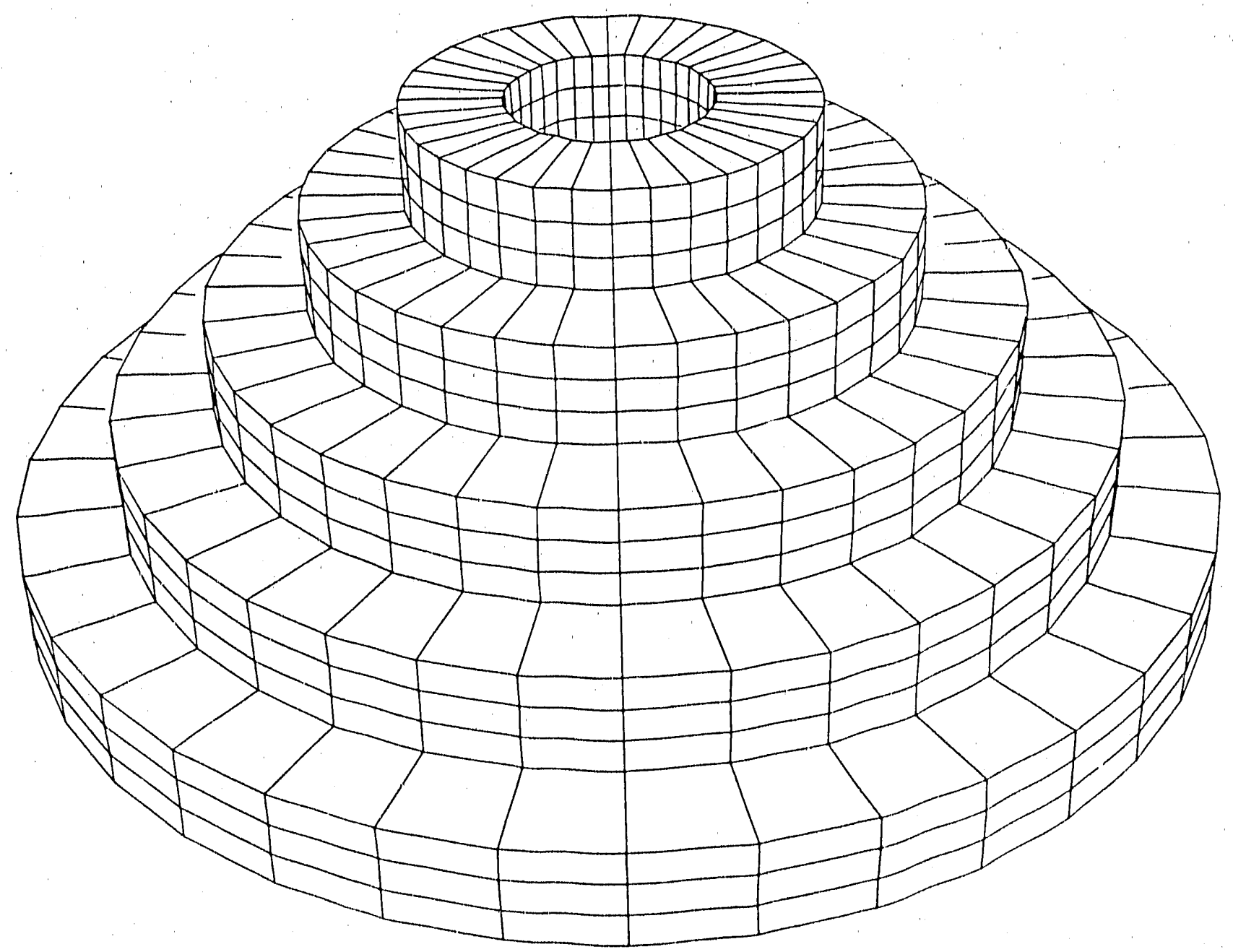


simple solid sphere section dn3d

start

1 4:1 37:1 21:

$\begin{array}{llllll}1.820 & 0 & 180 & -80 & 80\end{array}$

sphe

end

end

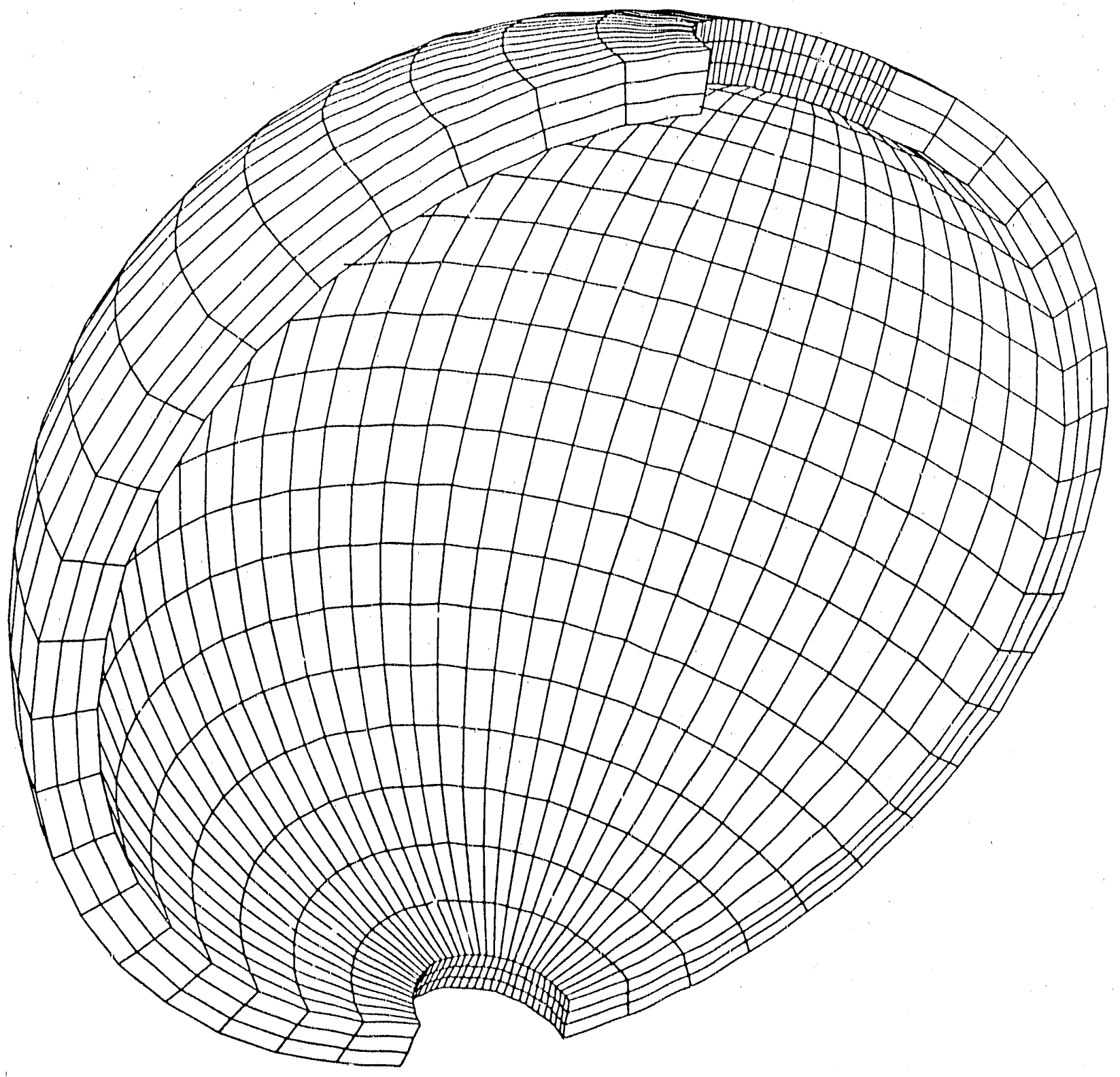


stair stepped spheres dn $3 d$

start

$\begin{array}{llllllllllllllllll}1 & 3 & 5 & 7 & 9 & 1 & 1 & : & 1 & 37 & : & 4 & 7 & 10 & 13 & 15 & :\end{array}$

$\begin{array}{lllllllllllllllll}1 & 1.1 & 1.2 & 1.3 & 1.4 & 1.5 & 0 & 180 & 0 & 15 & 30 & 45 & 60 & 75\end{array}$ sphe

d 20101302

d 300101400

d $4 \begin{array}{llllll}4 & 0 & 1 & 5 & 0 & 4\end{array}$

d 50010600

Ict 1 rXy:

Irep 0.1 ;

end

end

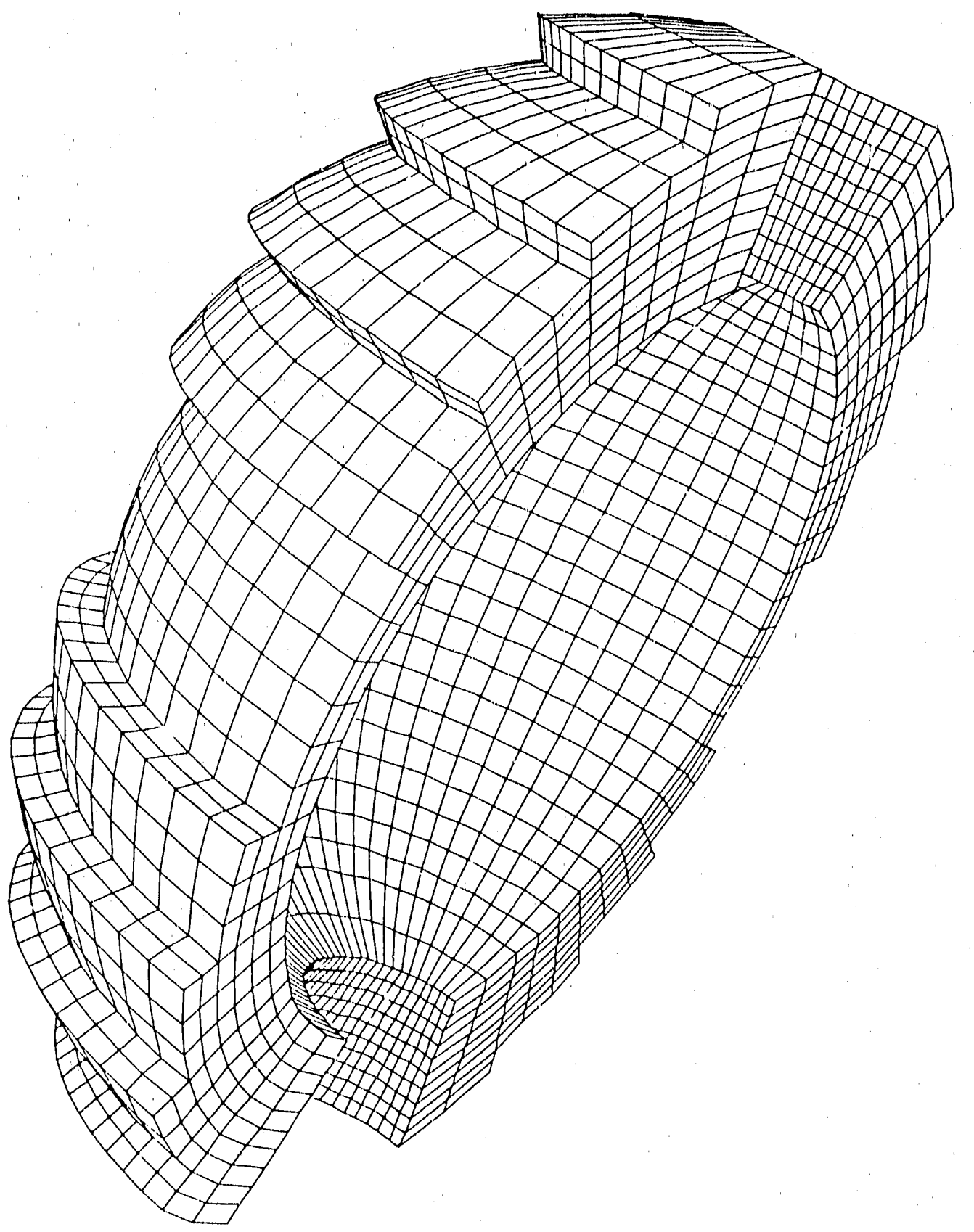


simple circular plate dn3d

start

$15: 15:-1$;

$\begin{array}{lllrllllll}-1 & 1 & -1 & 1 & 0 & & & & \\ 0 & 1 & 1 & 0 & 2 & 2 & 0 & 3 & 1.0\end{array}$

end

end

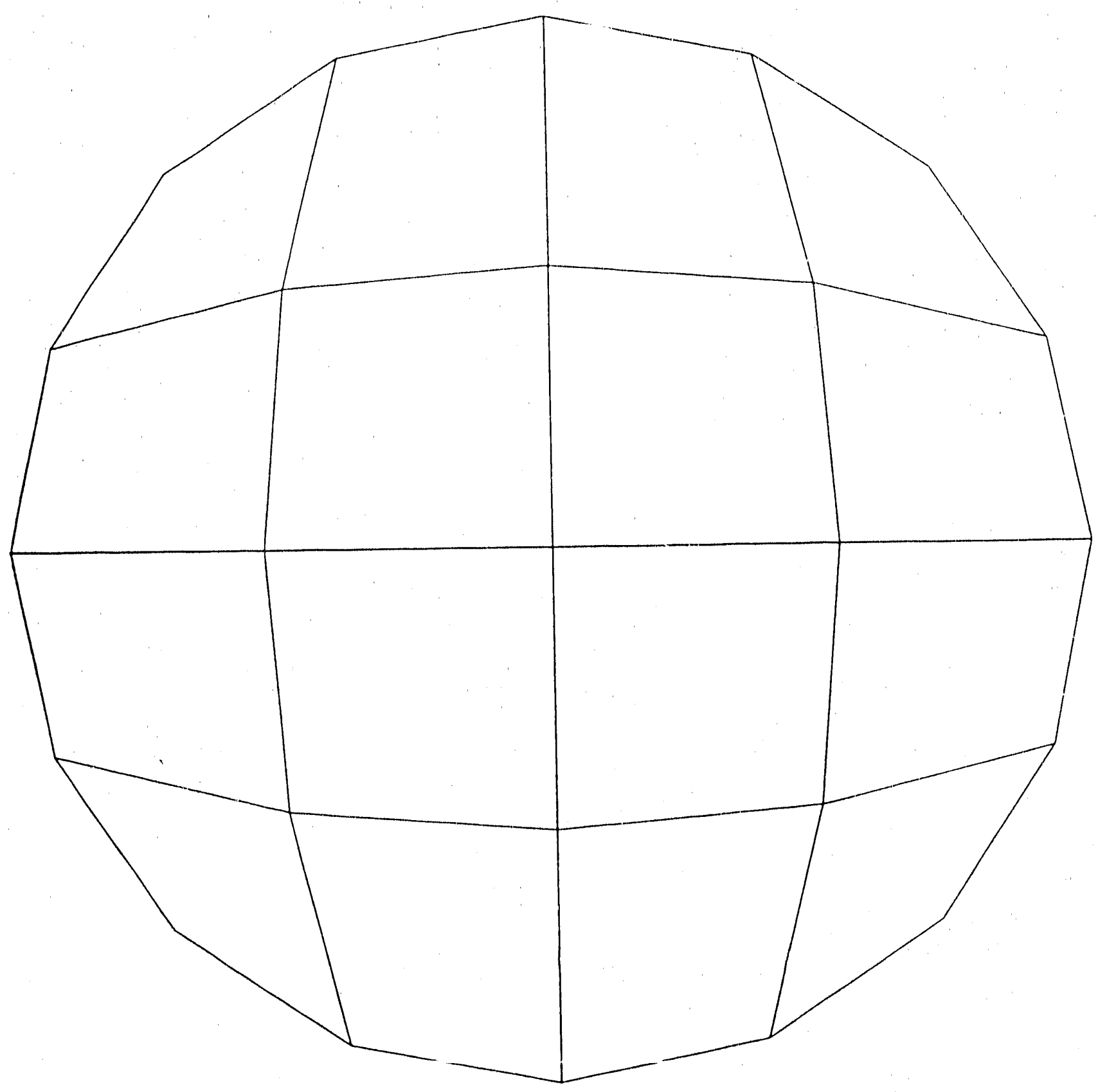


nicely zoned circular plate-solution \#1 dn $3 d$

\section{start}

$137 \quad 9: 137 \quad 3 \quad 9:-1$ :

$-.5-.5 .5 .5-.5-.5 .5 .50$

d 1110220

d 1300240

d 310.420

d $330044 \quad 40$

$\begin{array}{lllllllll}a & 1 & 1 & 0 & 4 & 4 & 0 & 3 & 1\end{array}$

end

end

nicely zoned circular. plate-solution \#2 din $3 d$

start

137 9:1 377 9:-1:

$-.5-.5 .5 .5-.5-.5 .5 .50$

di $120 \quad 34: 12034:-1 ;$

$\begin{array}{lllllllll}a & 1 & 1 & 0 & 4 & 4 & 0 & 3 & 1\end{array}$

end

end

nicely zoned circular plate-solution \#3 dn3d.

start

$1379: 137$ 9:-1:

$-.5-.5 .5 .5-.5-.5 .5 .50$

d $110220+12+j 2+i-2$

$\begin{array}{lllllllll}a & 1 & 1 & 0 & 4 & 4 & 0 & 3 & 1\end{array}$

end

end

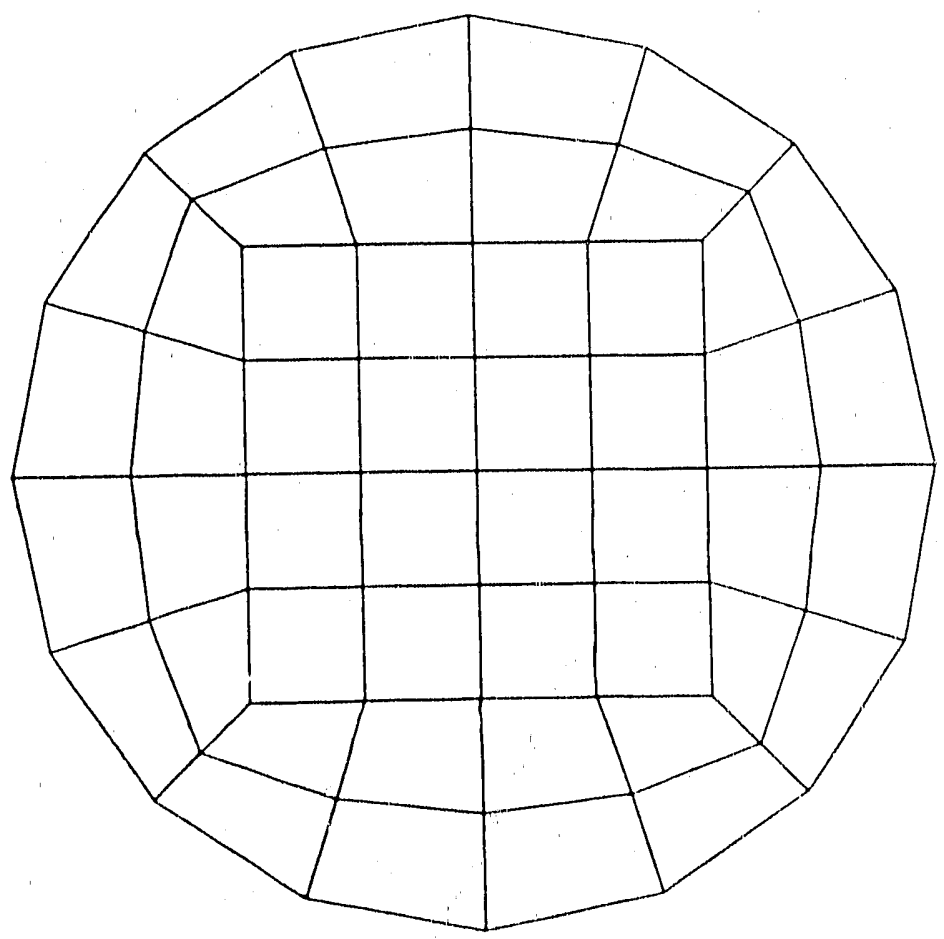


nalf circular plate - solution \#1 dn $3 d$

start

$13579: 1357$ 9: -1 :

$-.5-.50 .5 .5-.5-.50 .5 .50$

di $12045 ; 12045:-1$ :

a. 11100505001

d 1000300

end

end

half circular plate - solution \#2 dn3d

start

$135: 13.7 \quad 9:-1$;

$0.5 \quad .5-.5-.5 \quad .5 .50$

d. $23: 12034 ;-1$ :

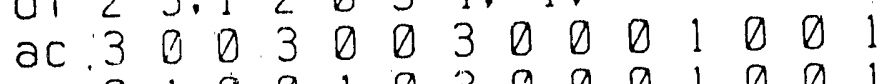
$\begin{array}{lllllllllllllll}a c & 0 & 1 & 0 & 0 & 1 & 0 & 3 & 0 & 0 & 0 & 1 & 0 & 0 & 1 \\ \text { ac } & 0 & 4 & 0 & 0 & 4 & 0 & 3 & 0 & 0 & 0 & 1 & 0 & 0 & 1\end{array}$ end end

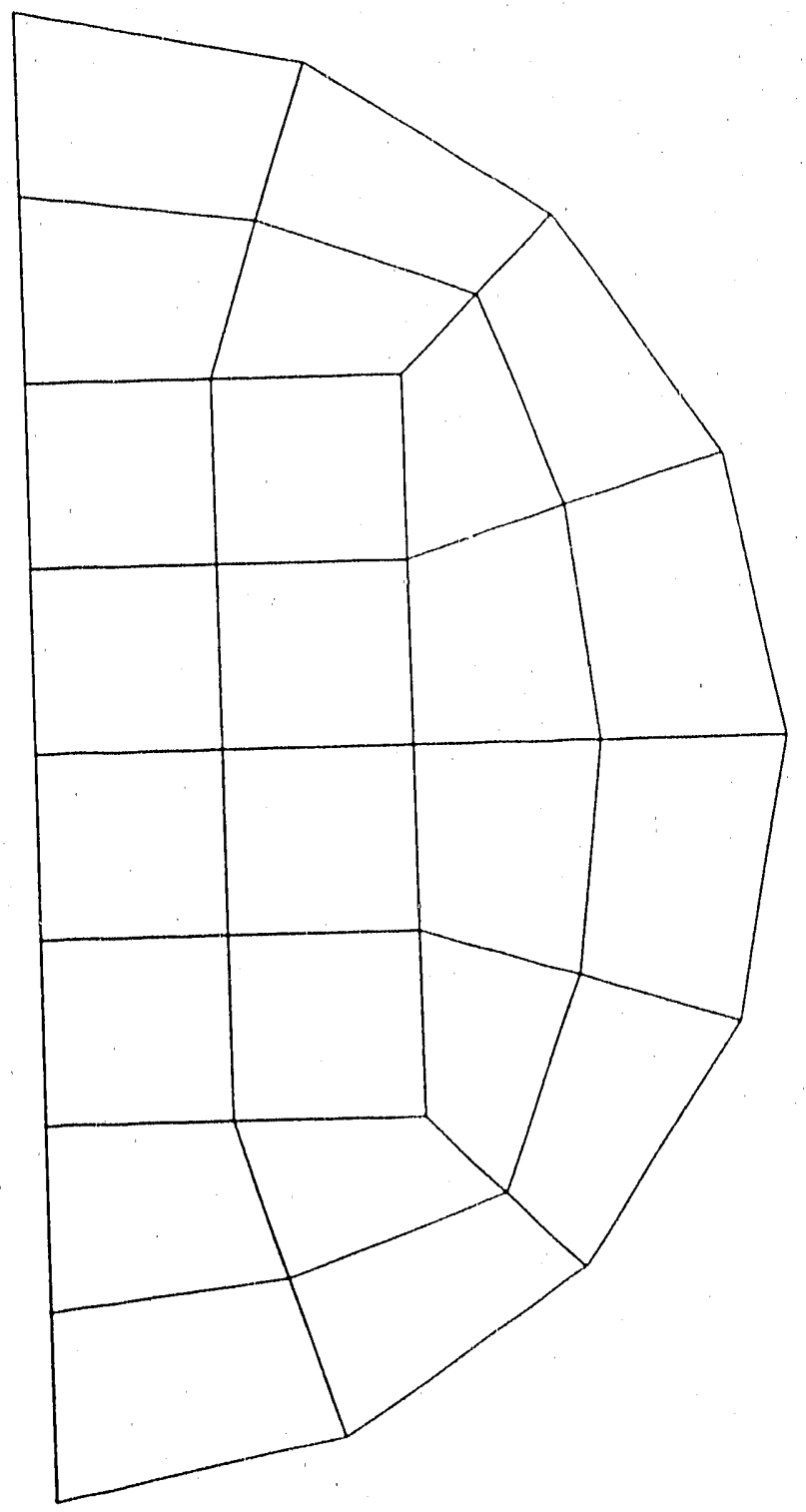


quarter circular plate-solution \#1

dn $3 d$

start

$135: 135:-1$;

0.5 .50 .5 .50

d. 220330

$\begin{array}{lllllllllllllll}a c & 3 & 0 & 0 & 3 & 0 & 0 & 3 & 0 & 0 & 0 & 1 & 0 & 0 & 1\end{array}$

$\begin{array}{lllllllllllllll}a c & 0 & 3 & 0 & 0 & 3 & 0 & 3 & 0 & 0 & 0 & 1 & 0 & 0 & 1\end{array}$

end

end

quarter circular plate-solution \#2 dn3d

start

$13579: 13579:-1$;

$-.5-.50 .5 .5-.5-.50 .50 .50$

di 120 4 5:120 20 5:-1:

a 11100500031

$\begin{array}{llllllll}d & 1 & 0 & 0 & 3 & 0 & 0\end{array}$

$\begin{array}{lllllll}d & 0 & 1 & 0 & 0 & 3 & 0\end{array}$

end

end

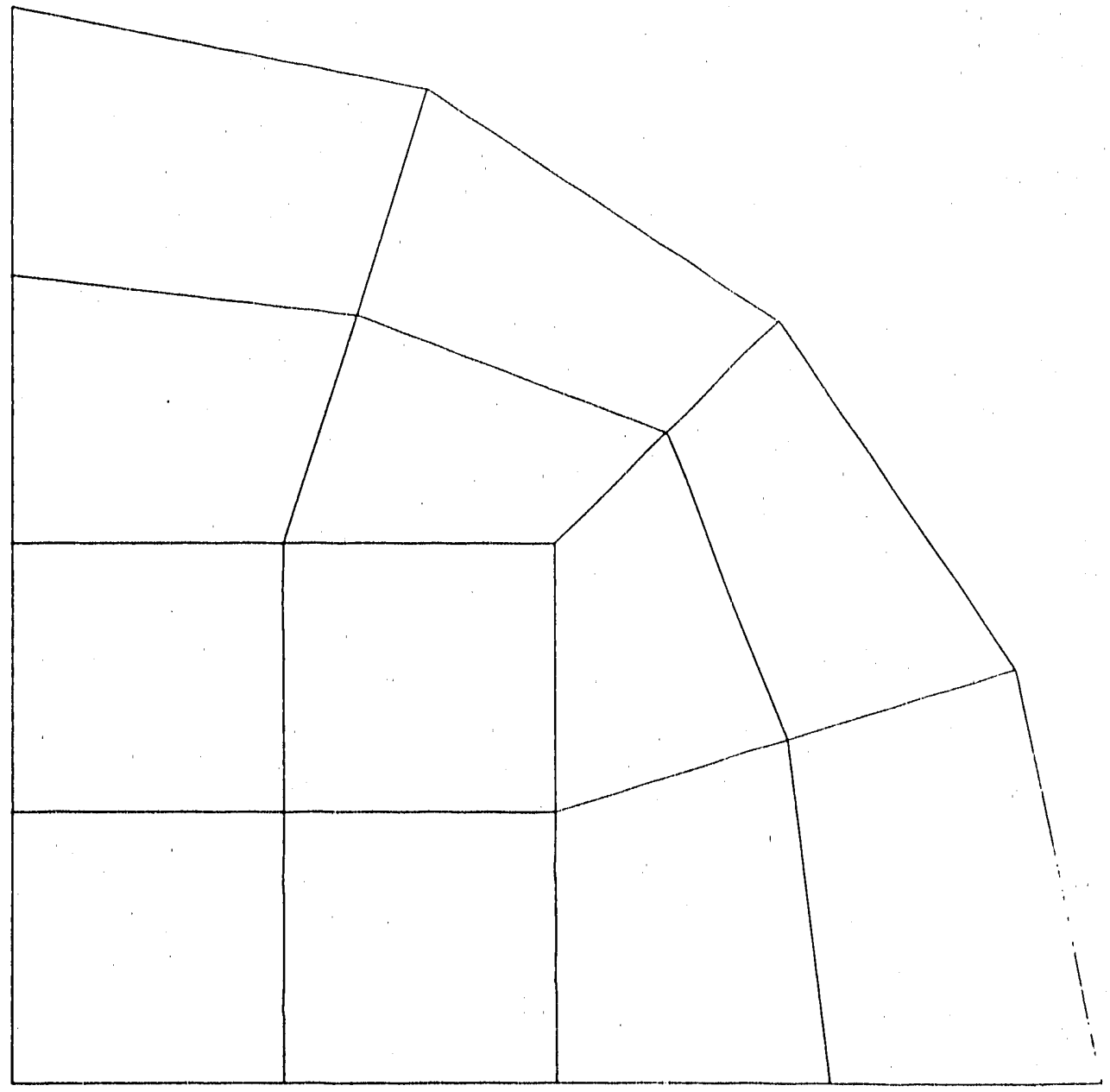


circular plate with outer liner dn $3 d$

mat 11 e $1.1 \mathrm{pr} 1.2$

head

moterial example\#1

mat 2 10 1.3 sigy 1.4 eh 1.5 al 1.6 npts 3

es $3.3 \quad 3.4 \quad 3.5$

eps $4.3 \cdot 4.4 \quad 4.5$

p 5.35 .45 .5

head

mater ial example \# 2

endmat

start

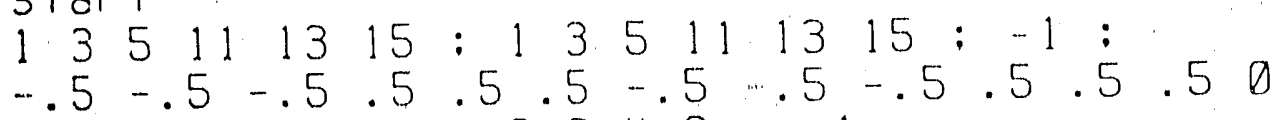

di $13046 ; 13046:-1$ :

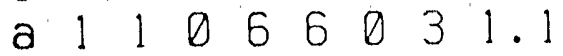

a 2220055001

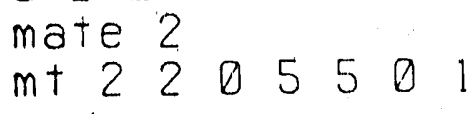

enc

enc

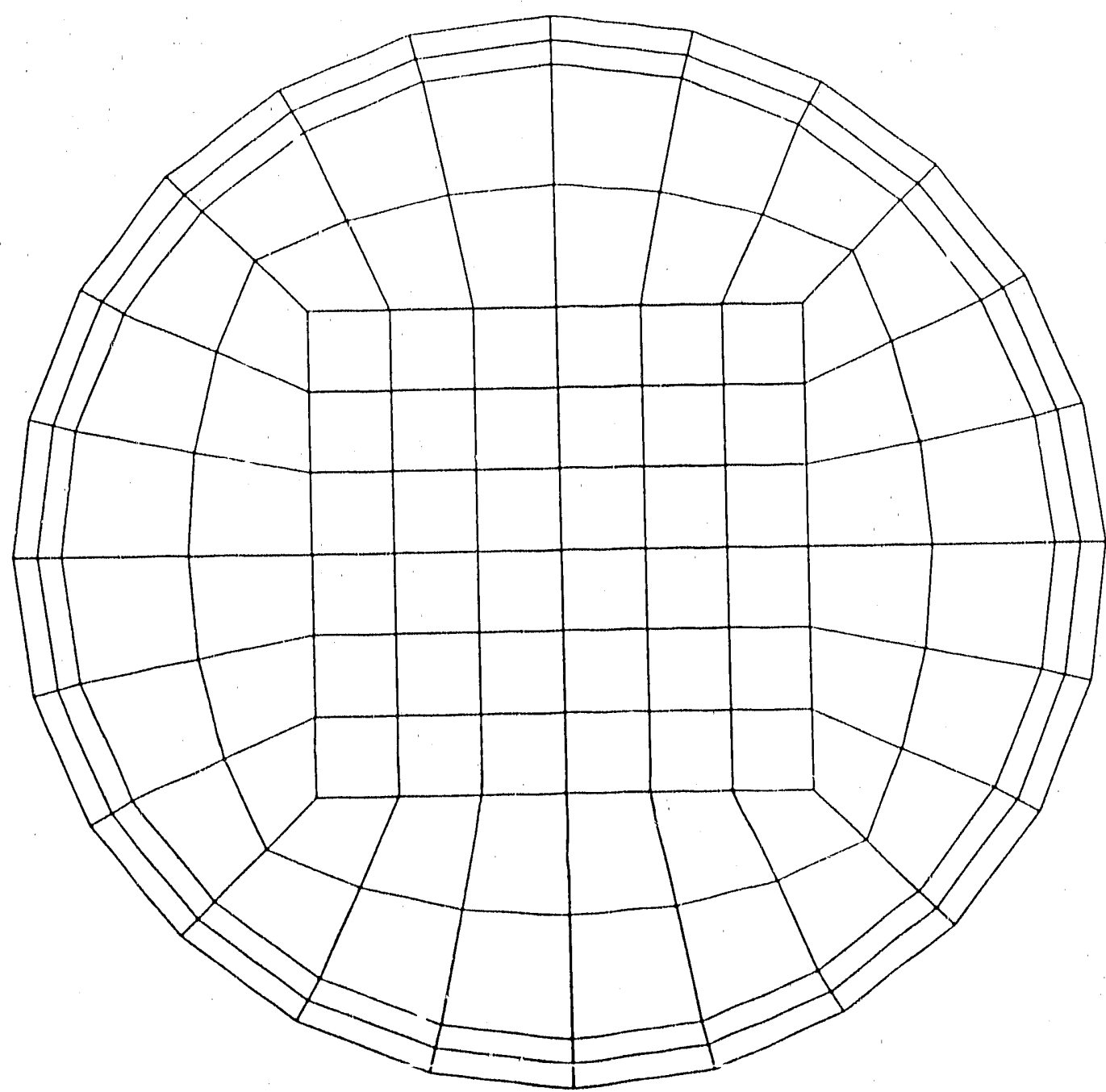


- circular plate with hole - solution\#1 dn $3 d$

start $13: 121:-1$ :

11.503600

cyli

end end

circular plate with hole - solution \#2 dn $3 d$

start $139911: 13911 ;-1$;

$\begin{array}{llllllllllllllllllllll}-1 & -1 & 1 & 1 & -1 & -1 & 1 & 1 & 0\end{array}$

di $12034: 12034:-1$ :

$\begin{array}{llllllllll}a & 1 & 1 & 0 & 4 & 4 & 0 & 3 & 1.5\end{array}$

d $2 \begin{array}{llllll}2 & 2 & 0 & 3 & 3 & 0\end{array}$

\begin{tabular}{llllllllll}
\hline & 2 & 2 & 0 & 3 & 3 & 0 & 3 & 1
\end{tabular}

end end

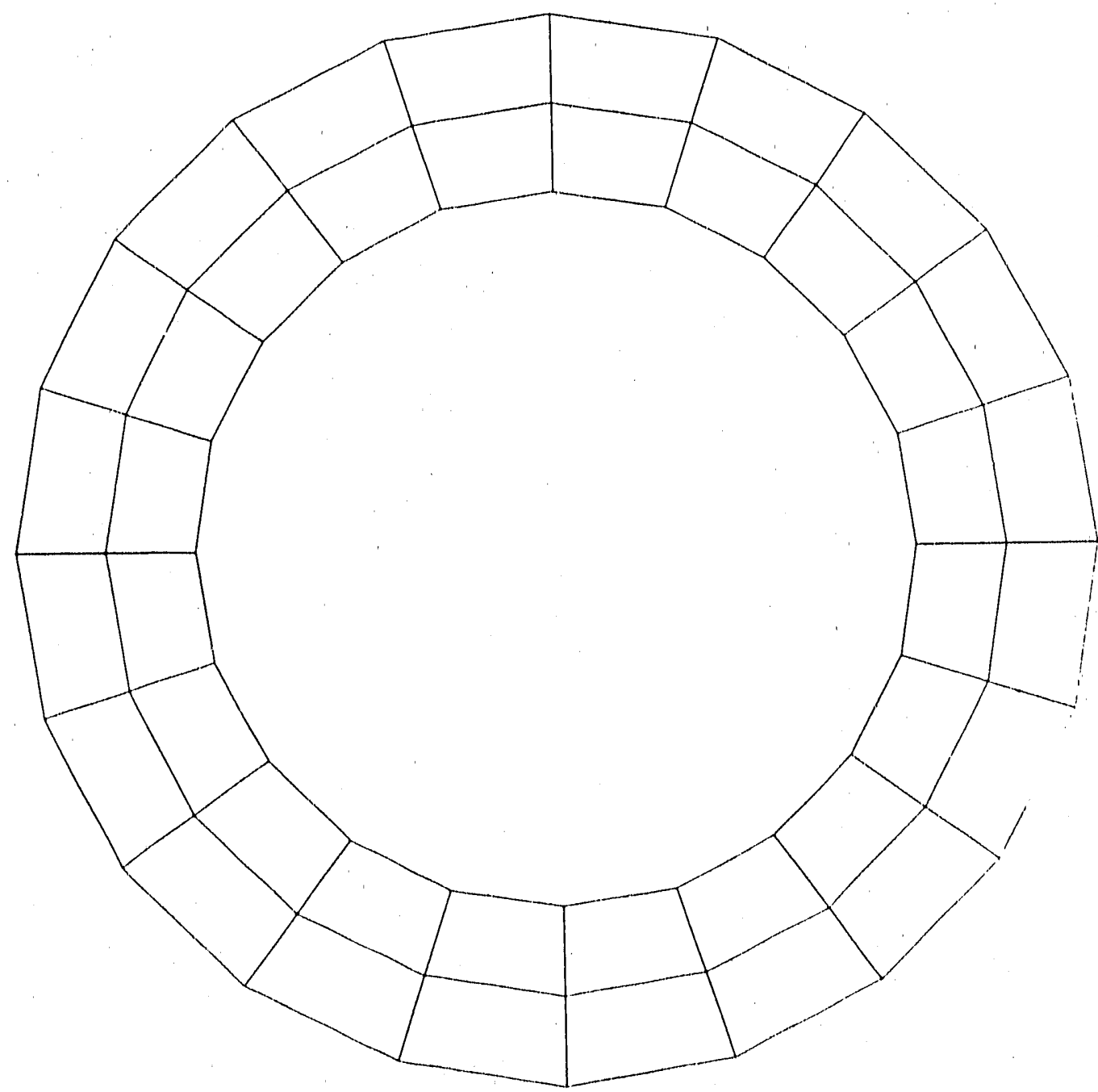


hollow shell cylinder - solution \# 1

$\operatorname{dn} 3 d$

start -1;1 19;1 5;

1.5036002

cyli

end end

hollow shell cylinder - solution \# 2 $\mathrm{dn} 3 \mathrm{~d}$

start -1 -5;-1 -5;1 5;

$-11-1102$

a 11022031.5

end end

hollow shell cylinder - solution \# 3

$\mathrm{dn} 3 \mathrm{~d}$

start $-1-5 ;-1-5 ; 15$;

$-11-11102$

sf 110220 cy 0000011.5

end end

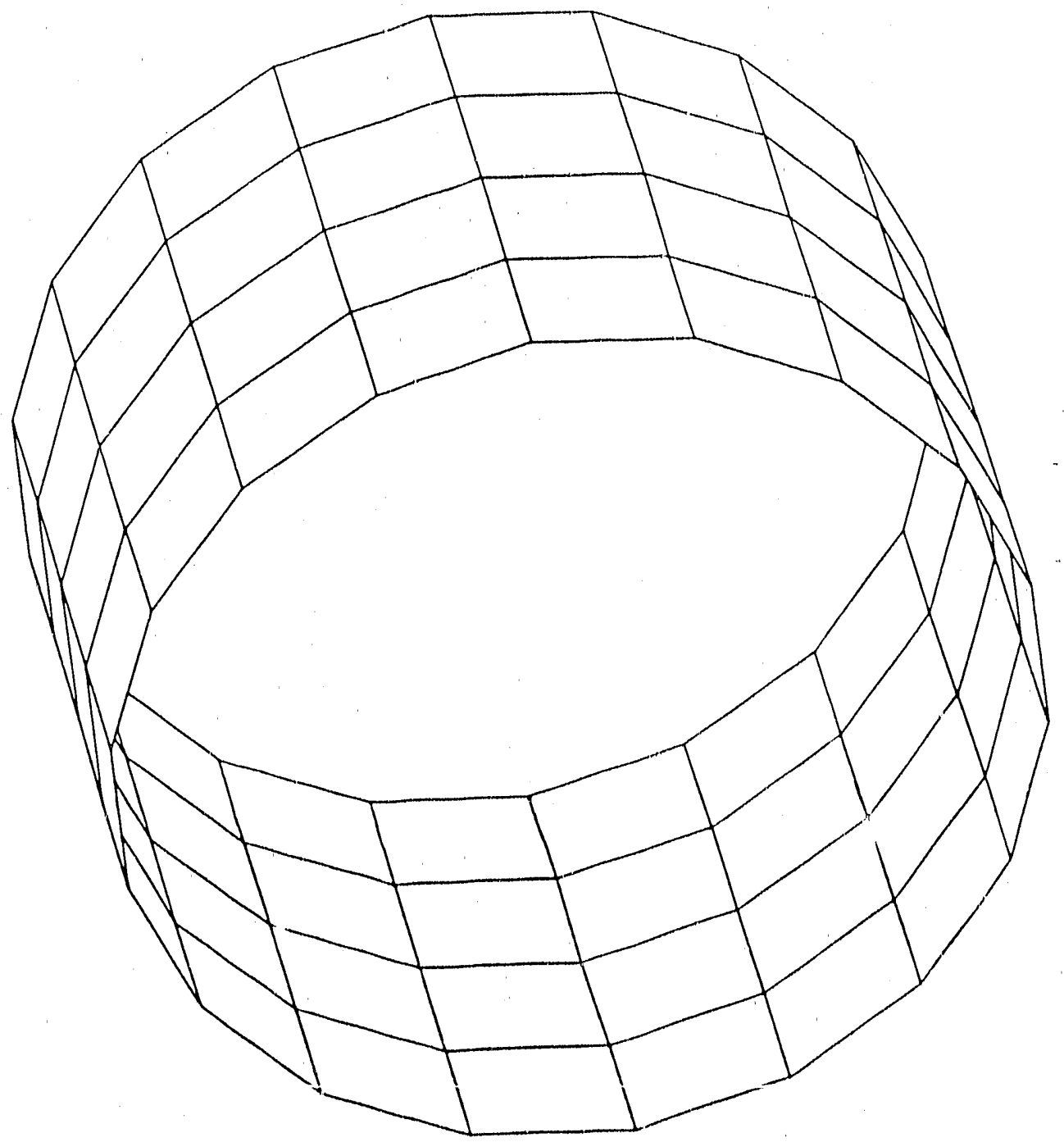


cylinder with stiffeners

\section{dn3d}

start 1 -3:1 19:-1 -5;

1.21 .5036002

cyli

end end

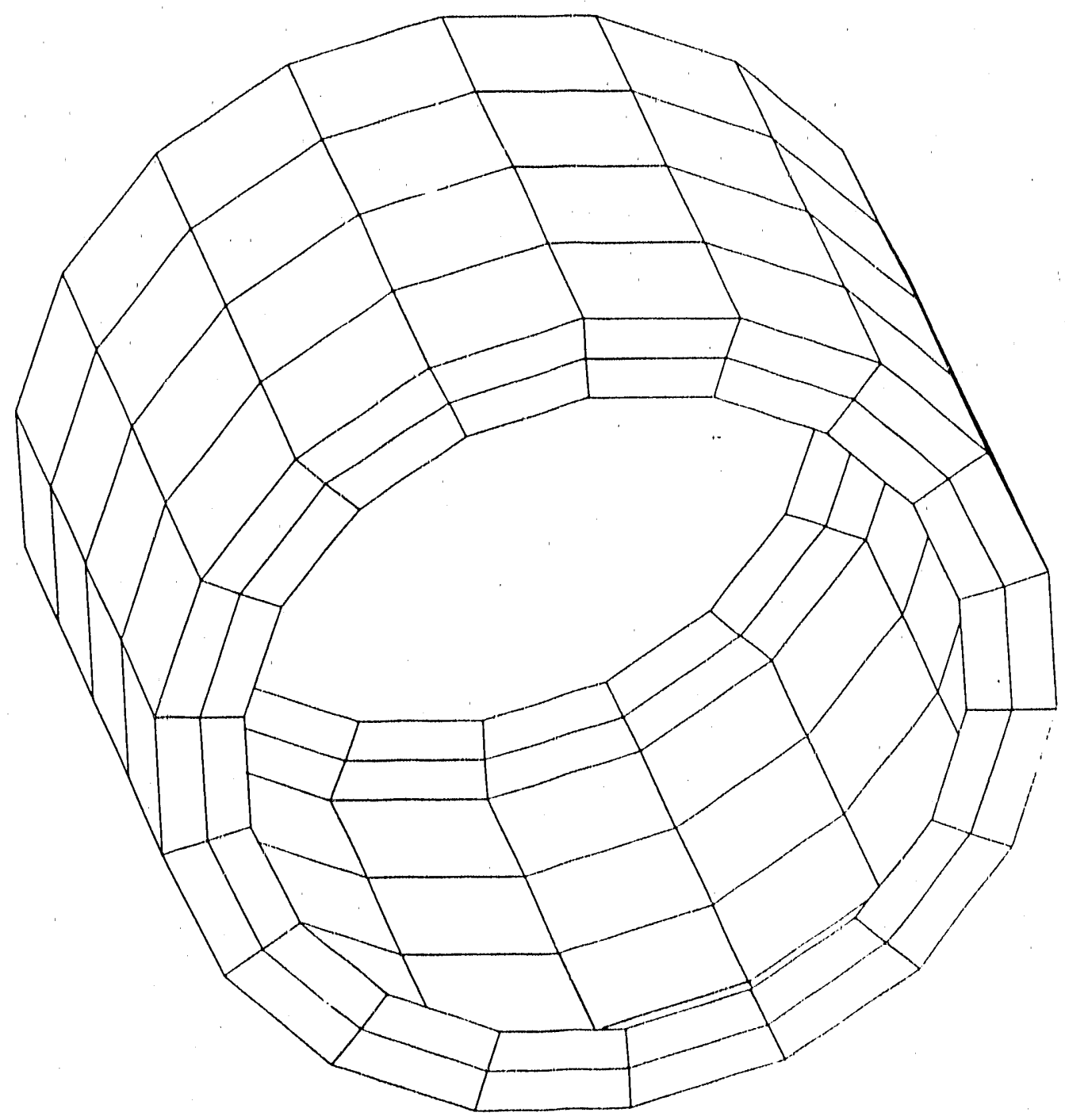


plate with hole in it dn $3 d$

start $15 \begin{array}{lllllllll} & 5 & 11 & 15: 1 & 5 & 11 & 15:-1 \text { : }\end{array}$

$\begin{array}{lllllllll}-1 & -1 & 1 & 1 & -1 & -1 & 1 & 1 & 0\end{array}$

di $120034: 12034:-1$ :

a: $1004 \quad 4 \quad 4 \quad 0 \quad 3 \quad 3$

$d=20330$

mb $22033012.75 \quad .75$

$a=203303.5$

eñ end

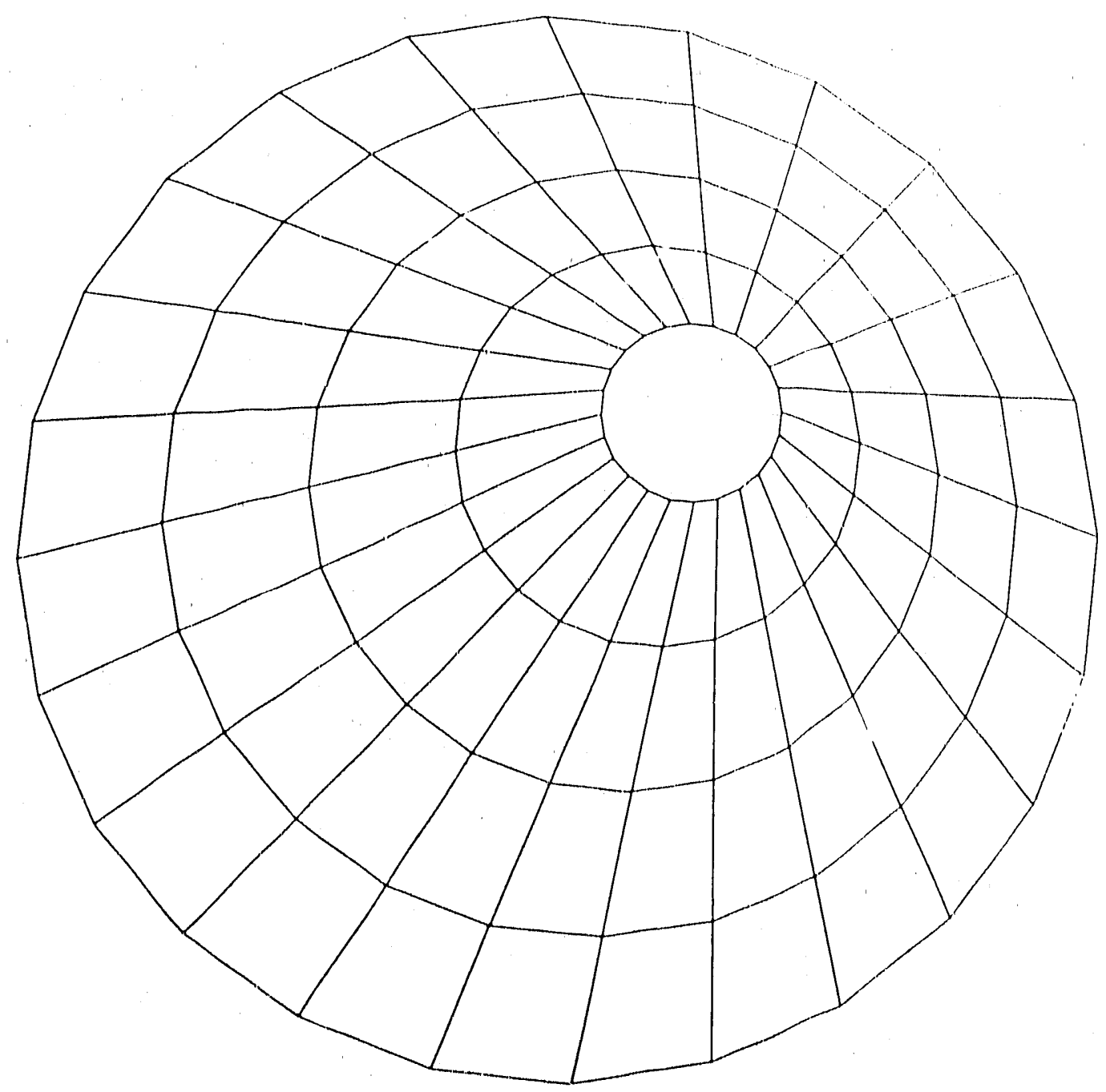


- square nlate with hole dn3d

start 159 13:1 5 9 13:-1:

$0 \begin{array}{llllllll}1 & 2 & 3 & 0 & 1 & 2 & 3 & 0\end{array}$

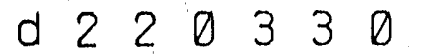

\begin{tabular}{llllllllll}
\hline & 2 & 2 & 0 & 3 & 3 & 0 & 3 & .75
\end{tabular}

end end

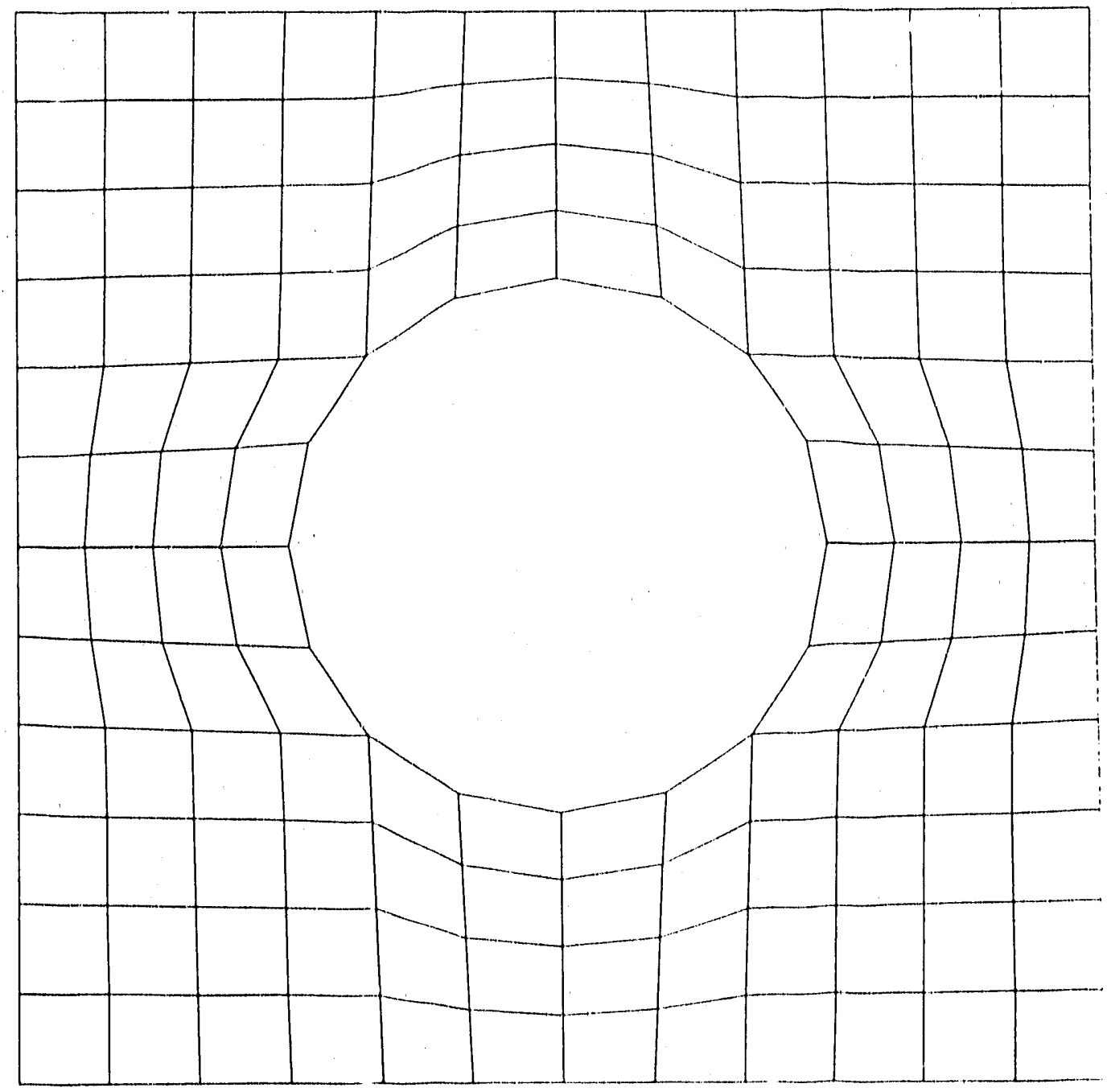


22
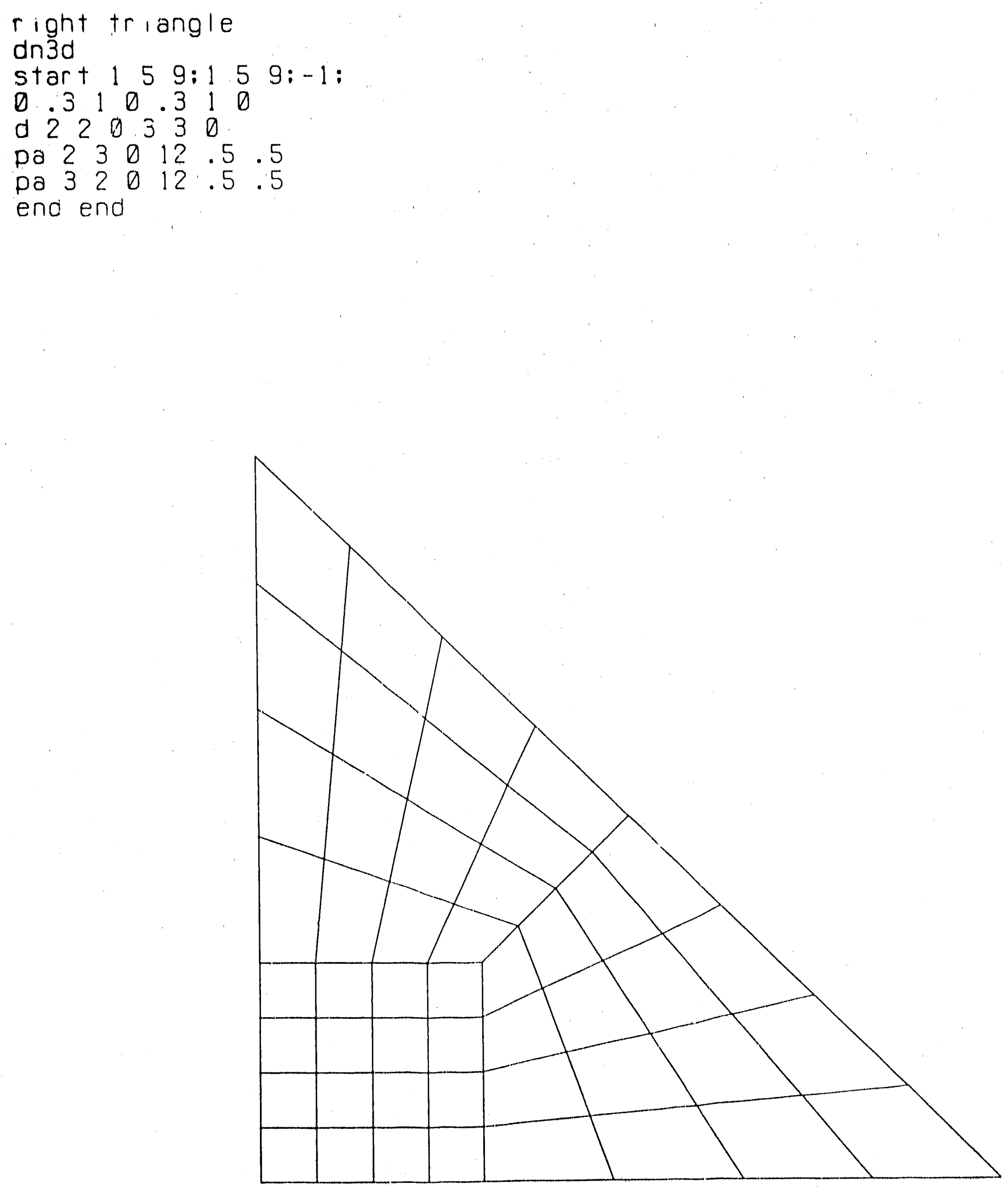
square plate with hole dn $3 d$

start 159 9 13:1 5 9 13:-1:

di $122^{2} \theta^{2} 3 \quad 4: 1^{-2} 2^{2} \theta^{2} 3^{-2} 4:-1$;

d $22 \quad 2 \quad 0 \quad 3 \quad 3 \quad 0$

$\begin{array}{llllllllll}a & 2 & 2 & 0 & 3 & 3 & 0 & 3 & 1.1\end{array}$

end end

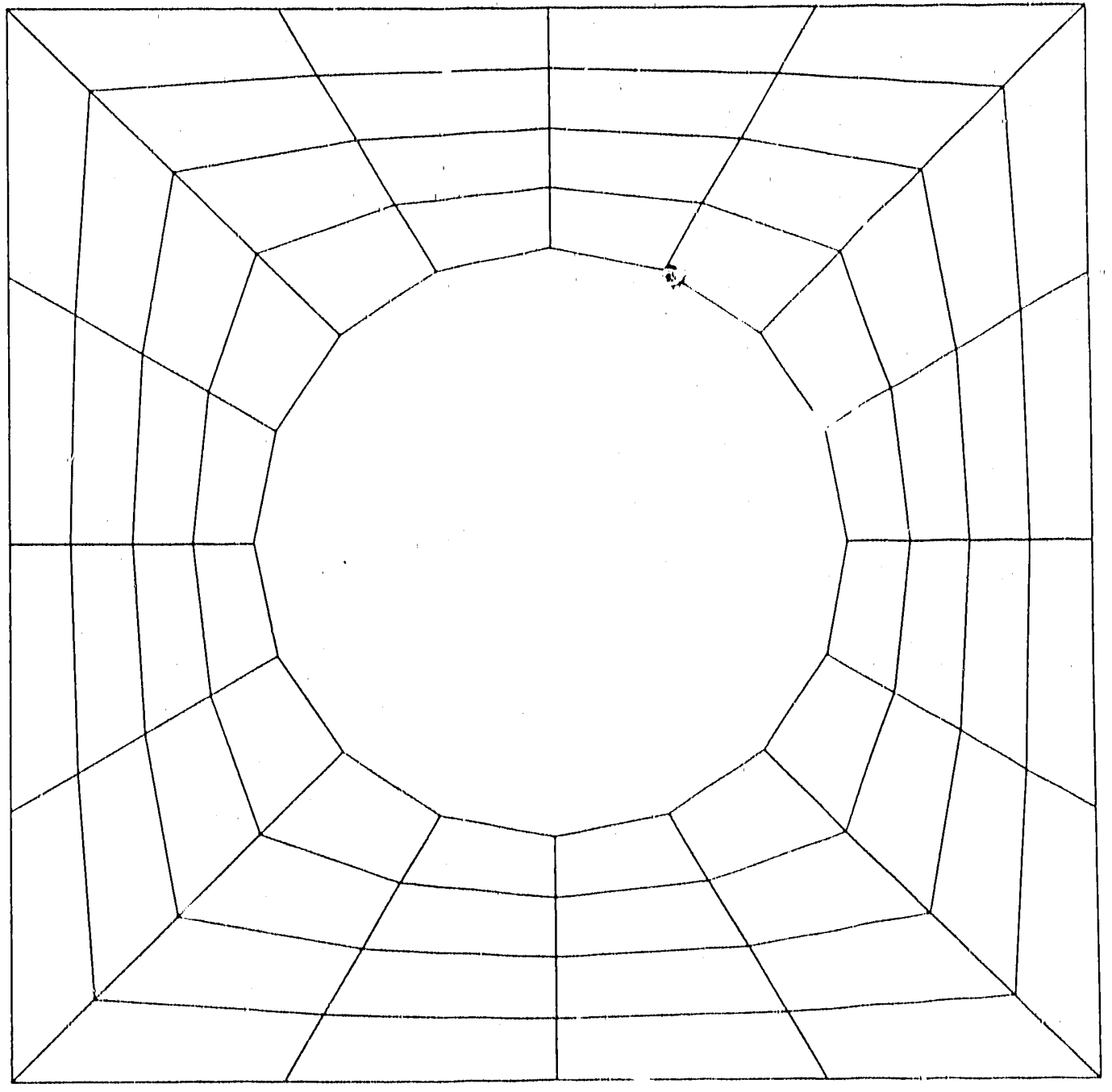


solid sphere

dn $3 d$

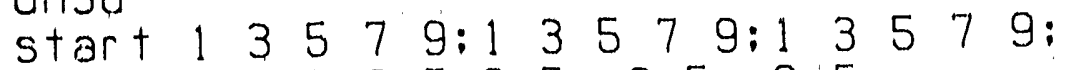

$\begin{array}{lllllll}-2.5 & -2.5 & 0 & 2.5 & 2.5 & -2.5 & -2.5\end{array}$

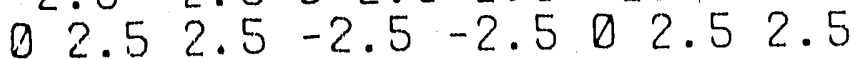

$d_{1} i 20045: 12004$ 5: :

di $12045:$ : 12 : $2045:$

$d_{1}: 12045: 12645:$

sf $1-1 \quad-5:-1 \quad-5:-1 \quad-5:$ sp 0000

0100300 end end

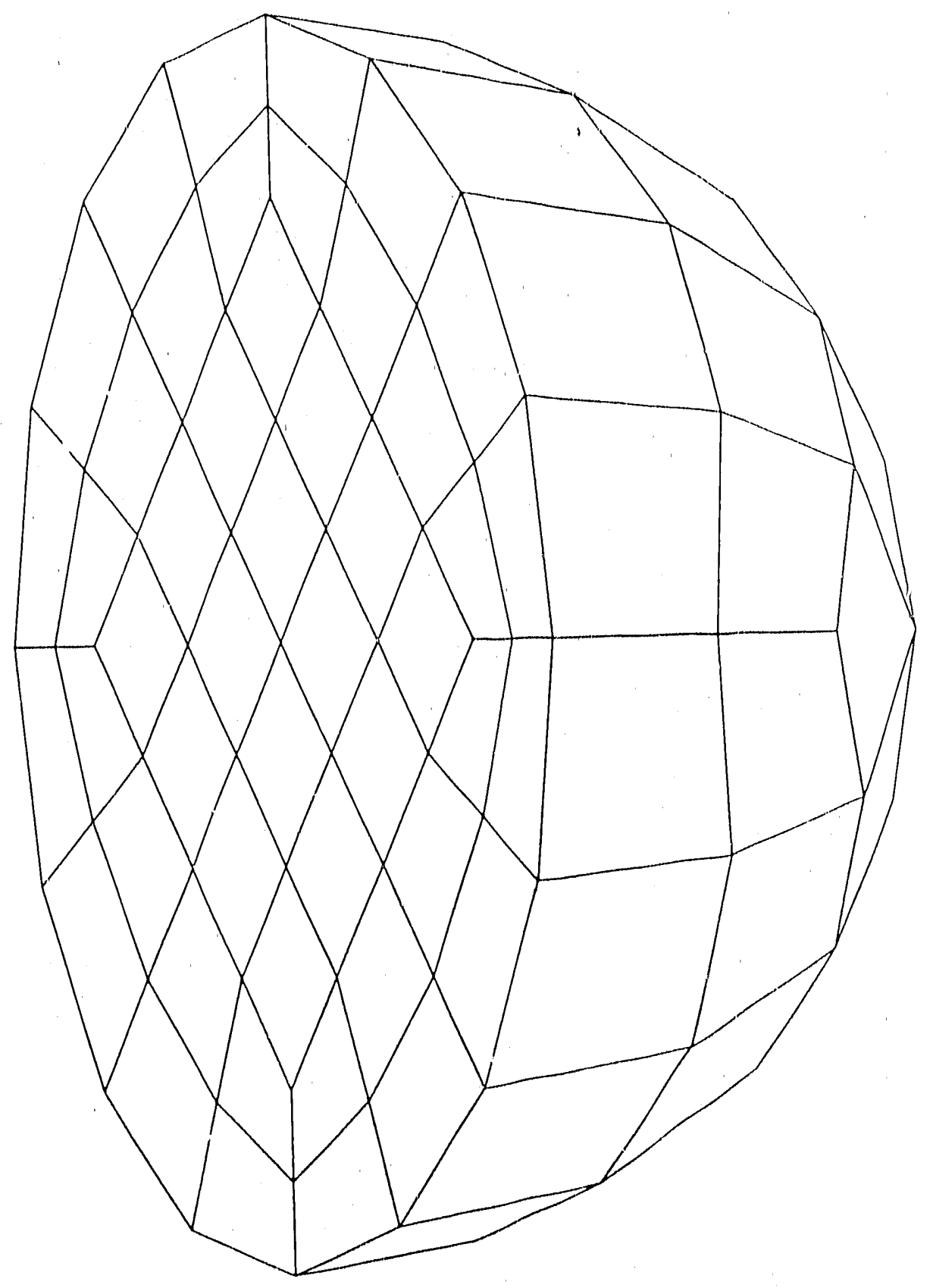


spherical shell - radius : 5.0 dn $3 d$

start $-1 \quad 5-9:-1 \quad 5 \quad-9 ;-1 \quad 5-9$;

$\begin{array}{llllllllll}-1 & 0 & 1 & -1 & 0 & 1 & -1 & 0 & 1\end{array}$

sf $1-1 \quad-3:-1 \quad-3:-1 \quad-3: s p \quad 0000$

d 100200

end end

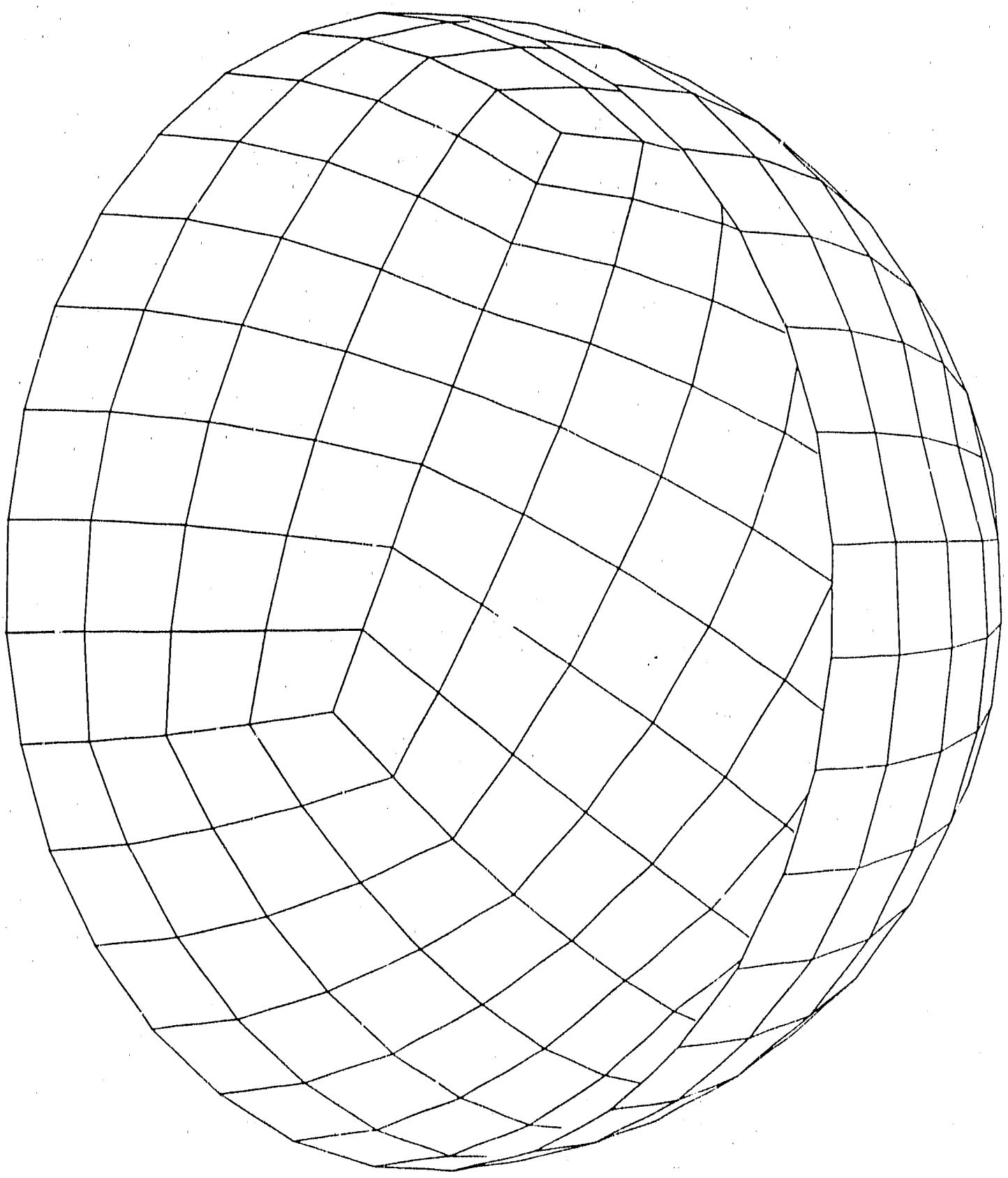


hich spherical shell-r1=3.0. ro: $=5.0$ $\ln 3 d$ tart 13577 9:1 $35579: 13579$; $\begin{array}{llllllllllllllll}1 & -1 & 0 & 1 & 1 & -1 & -1 & 0 & 1 & 1 & -1 & -1 & 0 & 1 & 1\end{array}$ 1i $120045: 120045:$

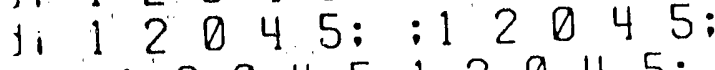

$1_{1}: 120045: 12045:$

$\begin{array}{lllllllll}\text { if } 1 & -1 & -5:-1 & -5:-1 & -5: \text { sp } & 0 & 0 & 0 & 5 \\ \text { if } 1 & -2 & -4:-2 & -4:-2 & -4: \text { sp } & 0 & 0 & 0 & 3\end{array}$

y 222444

$\begin{array}{lllllll}1 & 0 & 0 & 3 & 0 & 0\end{array}$

?nd end

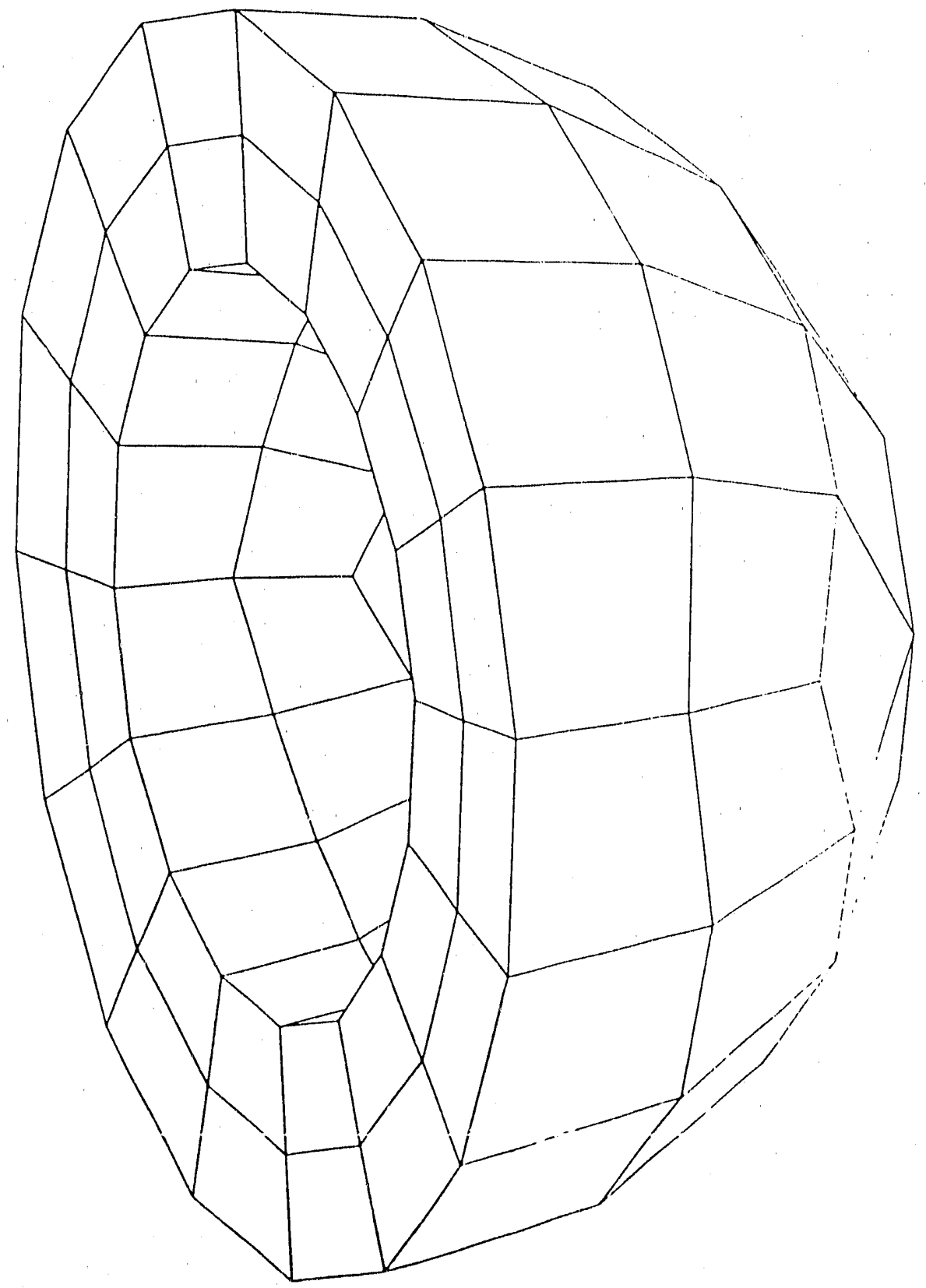


solid cylinder with spherical end - radius: 5.0 dn3d

$\therefore$ start $13579: 13579: 1579$;

$\begin{array}{llllllllllll}-2.5 & -2.5 & 0 & 2.5 & 2.5 & -2.5 & -2.5 & 0 & 2.5 & 2.5\end{array}$

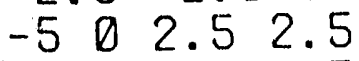

di $12045: 12045:$;

di $12045:$ : 3 4;

di $: 12045: 34$;

sf: $-1 \quad-5:-1 \quad-5: 1 \quad 2: c y \quad 0 \quad 0 \quad 0 \quad 00015$

sfi $-1 \quad-5:-1 \quad-5: 2 \quad-4: s p$ D 0.0 .5

d 1000000 end end

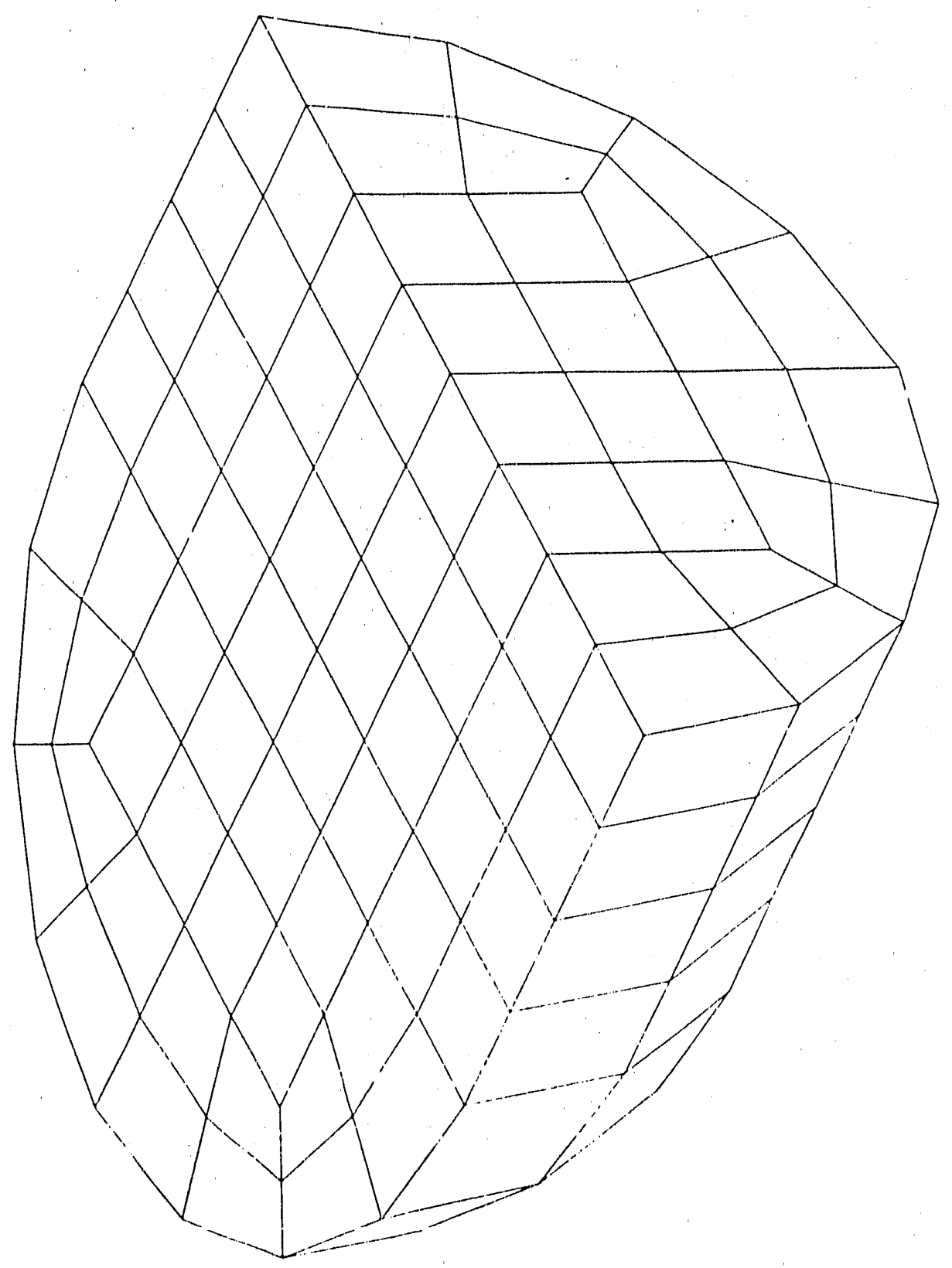


solid sphere with liner-r1:5.0. r2:5.5

dn $3 d$

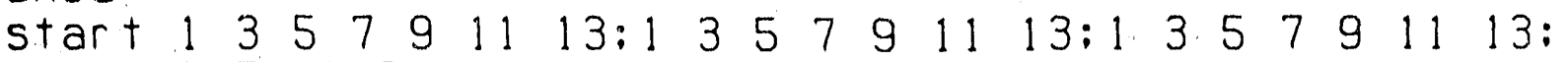

$-2.5-2.5-2.5 \quad 0 \quad 2.5 \quad 2.52 .5$

$\begin{array}{lllllll}-2.5 & -2.5 & -2.5 & 0 & 2.5 & 2.5 & 2.5 \\ -2.5 & -2.5 & -2.5 & 0 & 2.5 & 2.5 & 2.5\end{array}$

di $130557: 13057:$ :

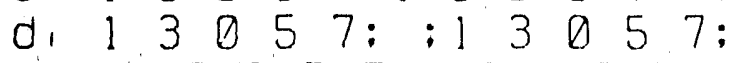

d. : $: \begin{array}{lllllllll} & 3 & 0 & 5 & 7: 1 & 3 & 0 & 5 & 7:\end{array}$

S: $-2-6:-2-6:-2-6: s p \quad 0 \quad 0 \quad 0.5$

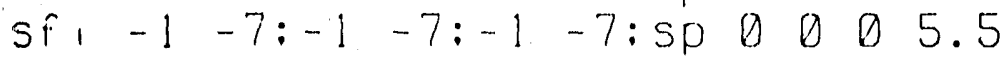

d 1000400 end end

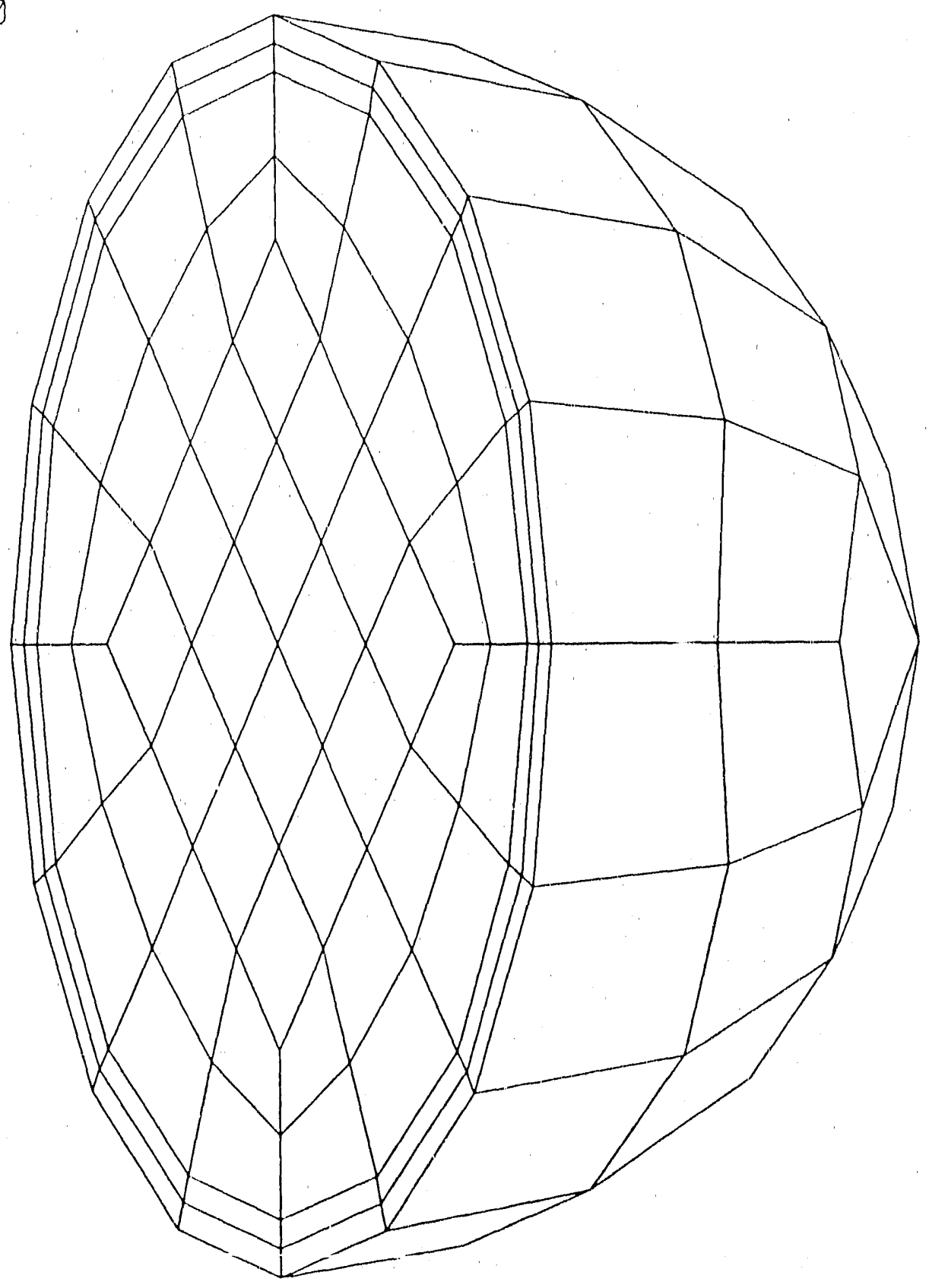



cylinder with spherical cap ${ }^{29}$ radius $=5.0$
dn3d

start $-1 \quad 5 \quad-9:-1 \quad 5-9: 1 \quad 5 \quad-9$;

$\begin{array}{llllllllll}-1 & 0 & 1 & -1 & 0 & 1 & -3 & 0 & 1\end{array}$

sf $1-1 \quad-3:-1 \quad-3: 1 \quad 2: c y 000000010$

sf $1-1 \quad-3:-1 \quad-3: 2 \quad-3: \operatorname{sp} \theta 000$

d 100200

end end

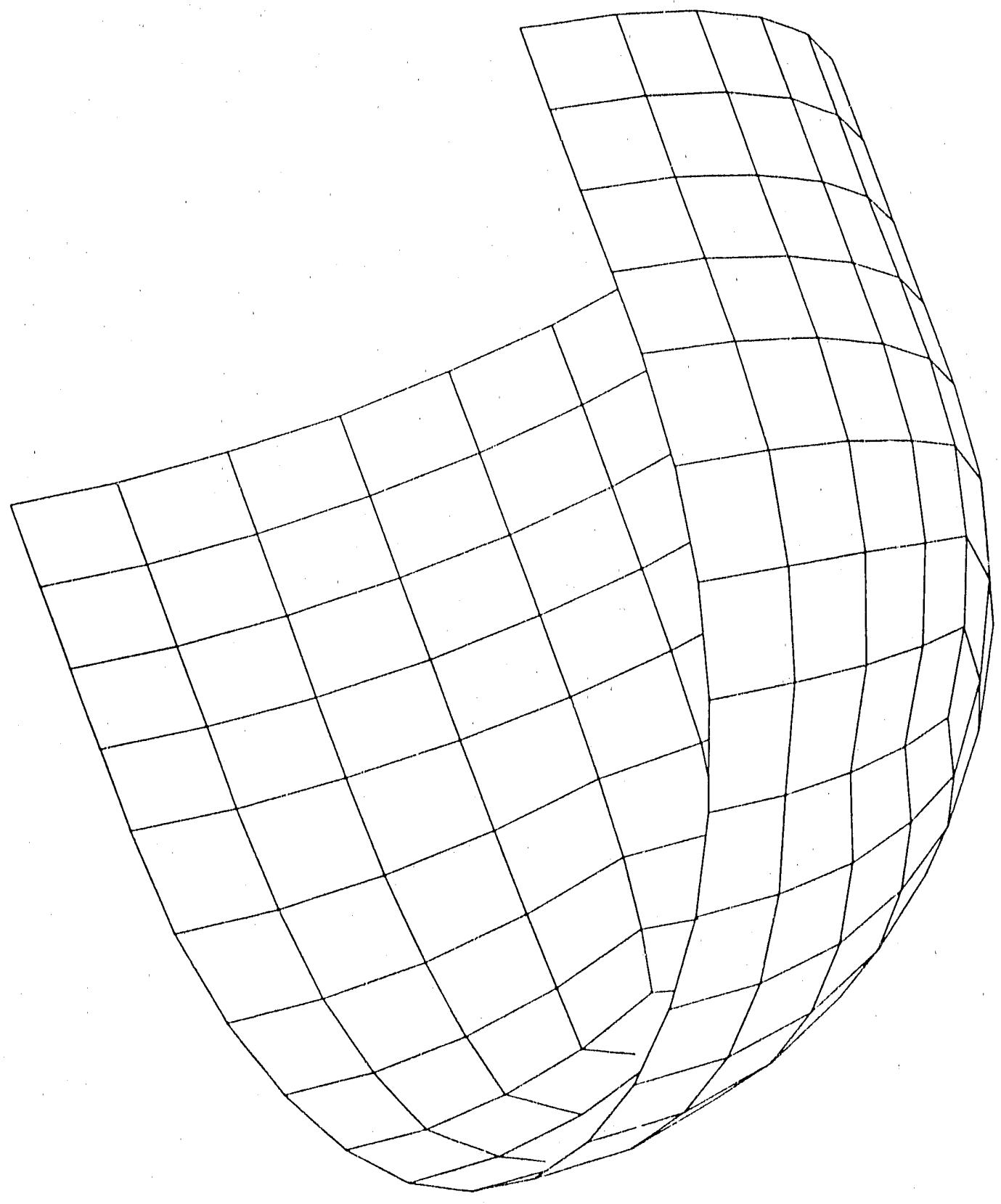


solid cylinder with spher ical end and $\mid$ iner-r $1=5.0, r 2=5.5$ dn3d

start.

$135577091113:$

17901113 :

$\begin{array}{lllllllll}-2.5 & -2.5 & -2.5 & 0 & 2.5 & 2.5 & 2.5 \\ -2.5 & -2.5 & -2.5 & 0 & 2.5 & 2.5 & 2.5\end{array}$

$-7.502 .52 .52 .5$

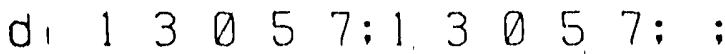

d: $13057:: 3$ : 3 :

di : $13057: 35$ :

sf, $-2-6:-2 \quad-6: 2 \quad-4:$ sp 00 0 005

sf $1-1 \quad-7:-1 \quad-7: 2 \quad-5: s p \quad 0 \quad 0 \quad 05.5$

sf $1-2 \quad-6:-2 \quad-6: 1 \quad 2: c y \quad 0 \quad 00000010$

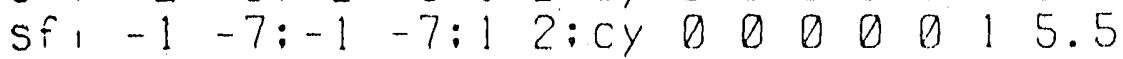

d 1000400

end end

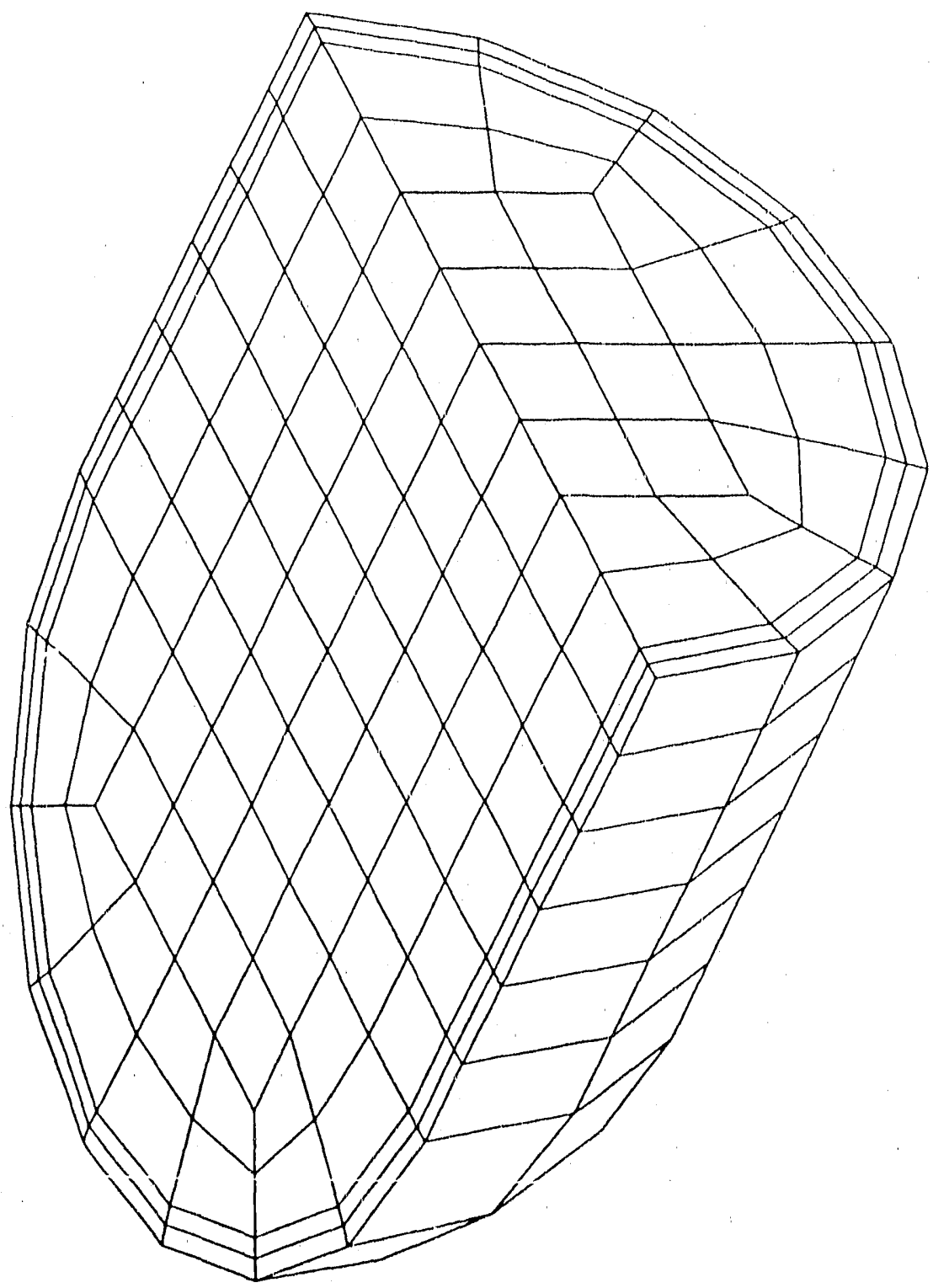


thick cylindrical shell with spherical cap-r 1=3.0.ro=5.0 dn $3 d$

start $13.579: 13579: 1579$ 9:

$\begin{array}{llllllllllllll}-1 & -1 & 0 & 1 & 1 & -1 & -1 & 0 & 1 & 1 & -2 & 0 & 1 & 1\end{array}$

di $12045: 12045:$;

di $120045:$ : 34 :

di $: 12045: 3$ 4:

sf $1-1-5 ;-1 \quad-5: 2-4: s p$ D

sf $1-2 \quad-4 ;-2 \quad-4 ; 2 \quad-3 ; s p$ D $\oslash 00$

sf $1-1 \quad-5:-1 \quad-5: 1 \quad 2: c y \quad 0 \quad 0 \quad 0 \quad 0 \quad 0 \quad 15$

Sf: $-2 \quad-4 ;-2 \quad-4 ; 1 \quad 2 ; c y \quad 0 \quad 000001013$

d $22 \begin{array}{lllll} & 4 & 4 & 3\end{array}$

$\begin{array}{lllllllllll}d & 1 & 0 & 0 & 3 & 0 & 0\end{array}$

end end

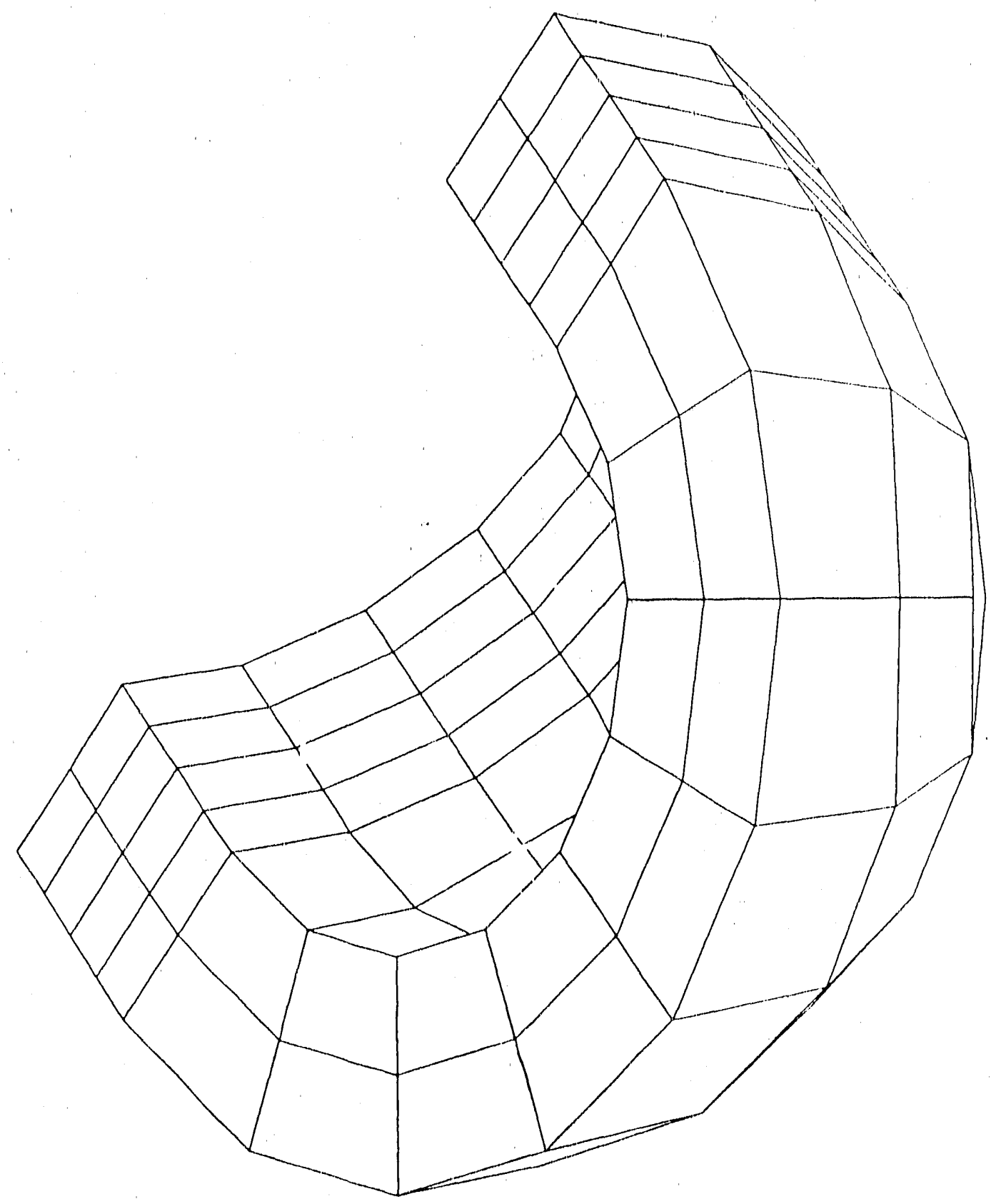


cylidrical shell with hole dn $3 d$

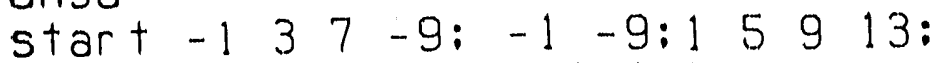

$\begin{array}{llllllllll}-2 & -1 & 1 & 2 & -2 & 2 & -3 & -1 & 1 & 3\end{array}$

d 20.2303

$\begin{array}{lllllllllllll}\text { sf: } & -1 & -4:-1 & -2: & \text { c cy } & 0 & 0 & 0 & 0 & 0 & 1 & 2 \\ \text { sf: } & -2 & -3: & :-2 & -3 ; & -3 & 0 & 0 & 0 & 0 & 1 & 0 & 1\end{array}$

$C C^{\prime} 1014011+k 31$

eriz end

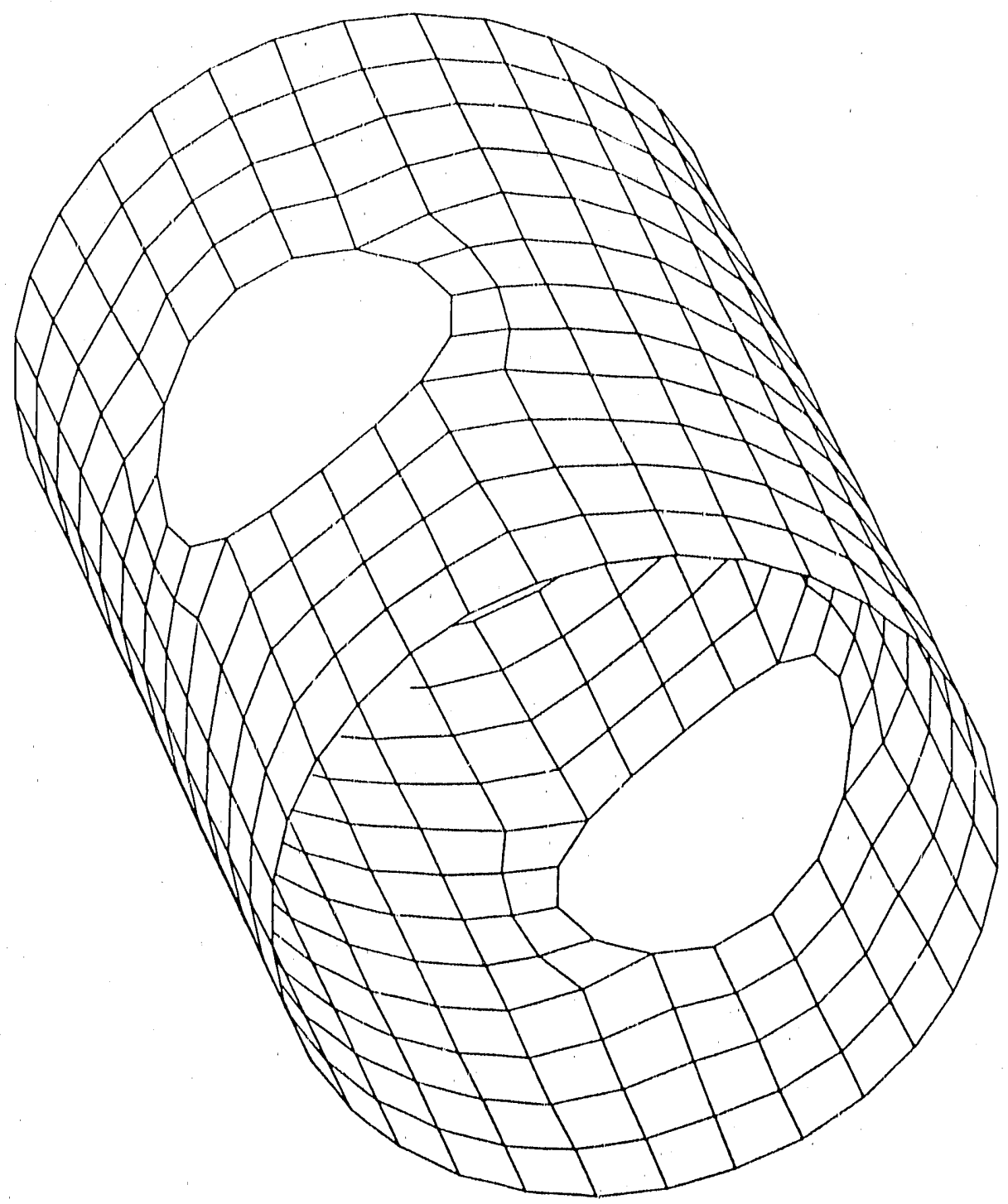


- cylidrical shell with hole and transition region dn $3 d$

start $-1-7:-1-7: 141013:$

$\begin{array}{llllllll}-1 & 1 & -1 & 1 & -3 & -2 & 2 & 3\end{array}$

di $;-1-2: 2 \quad 3 ;$

sf $1-1-2 ;-1-2 ; \quad ; c y \quad 0 \quad 0 \quad 0 \quad 00012$

end

start $17 \begin{array}{llllllll}7 & 13 & 19:-1 & -2: 1 & 7 & 13 & 19\end{array}$

$\begin{array}{llllllllll}-1 & -1 & 1 & 1 & -1 & 1 & -2 & -2 & 2 & 2\end{array}$

di $120034:-1-2: 12034:$

Sf $:-1-2: ; c y \quad 00000012$

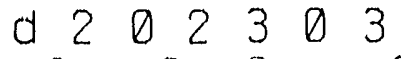

sf $1-2-3::-2 \quad-3: c y \quad 0 \quad 0 \quad 0 \quad 0 \quad 1 \quad 0 \quad .75$ end end

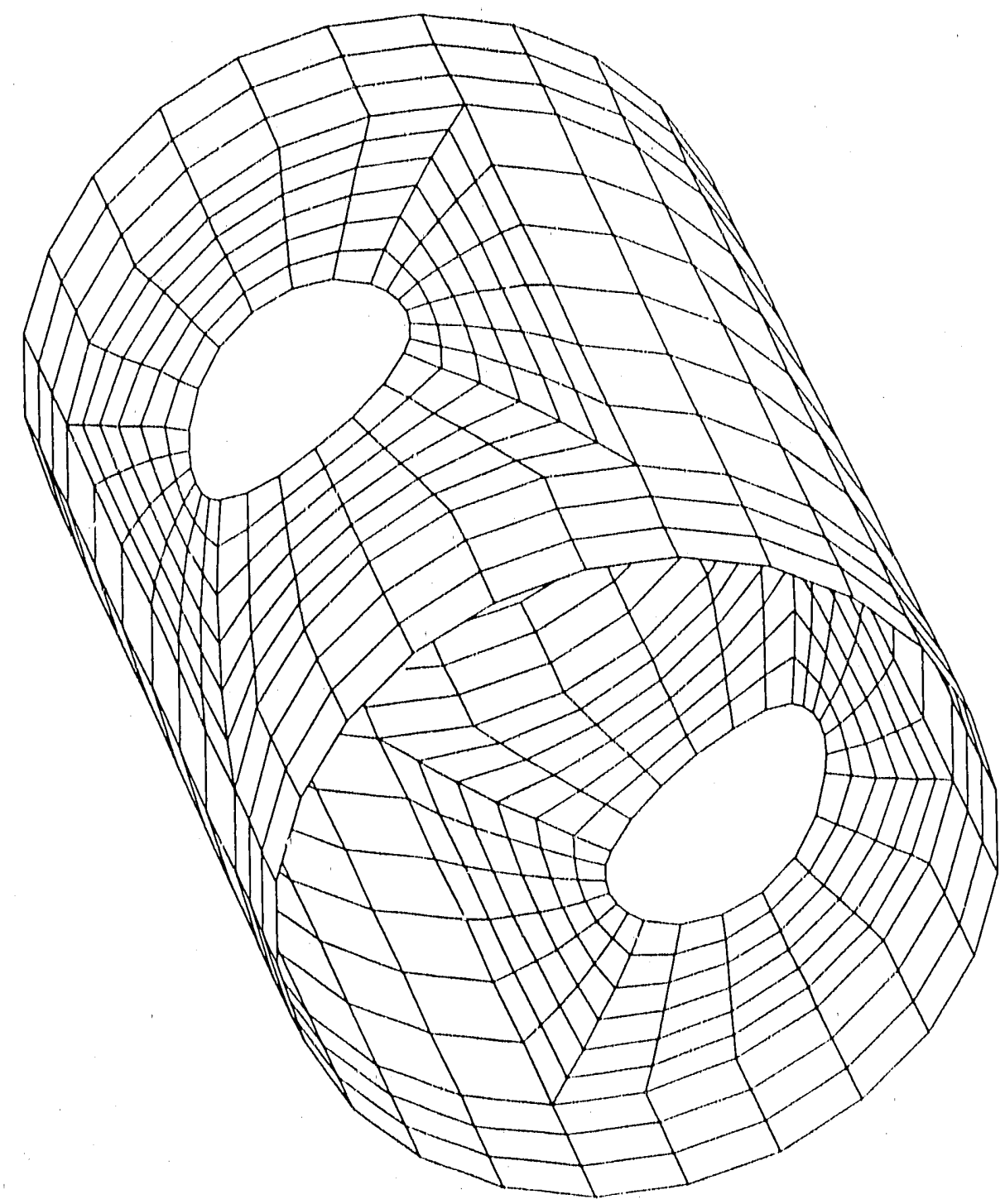


sphereical shell formed from a sheet dn3d

start $14 \quad 10 \quad 13: 14$ 10 13:-1:

$\begin{array}{lllllllll}-5 & -5 & 5 & 5 & -5 & -5 & 5 & 5 & 0\end{array}$

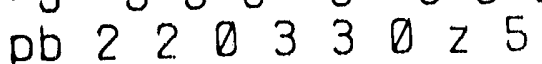

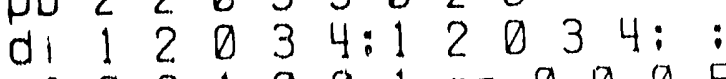

sf $\theta 0.1001$ sp $0 \quad 0 \quad 05$

end end

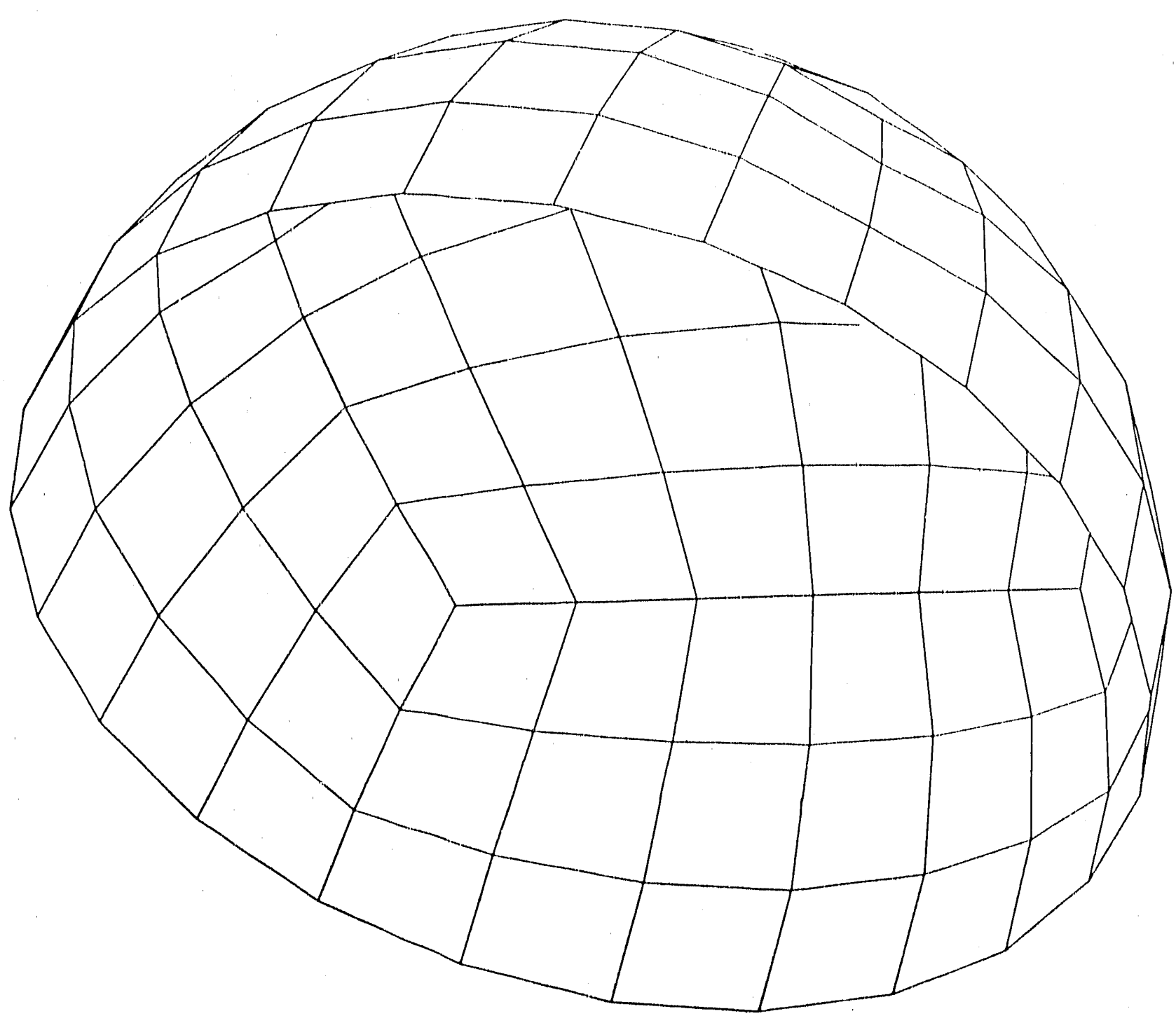


thick sper lcal shell formed from a sheet dn3d

start 1 4 10 13: 14 4 10 13:1 4:

$-10-101010-10-10101000$

$\mathrm{pb} 220330210$

di $12003 \quad 4: 120034 ;:$

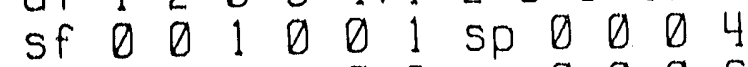

sf $\theta \quad 020 \quad 02 \operatorname{sp} \theta \quad 0 \quad 0 \quad 6$

end end

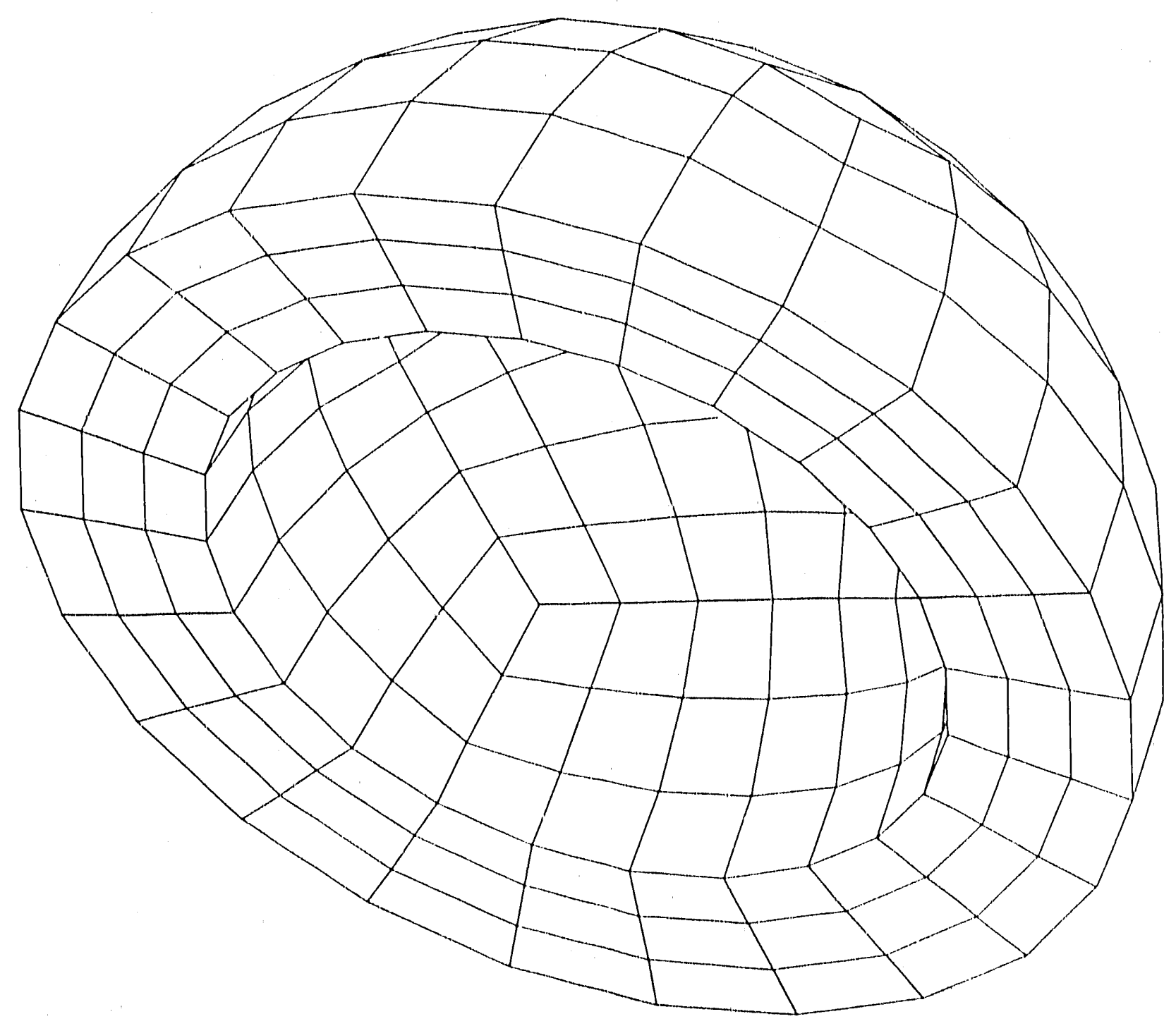


ylider with cylindrical cut out $\ln 3 d$ ; tart $-1 \quad 5 \quad-9:-1 \quad 5 \quad-9: 1559$ 13:

$202-202-6-226$

1202303

if $-1 \quad-3:-1-3 ;: c y \quad \theta \theta \theta \theta 012$

if -2 3: : $-2-3: c y 2000102$ and end

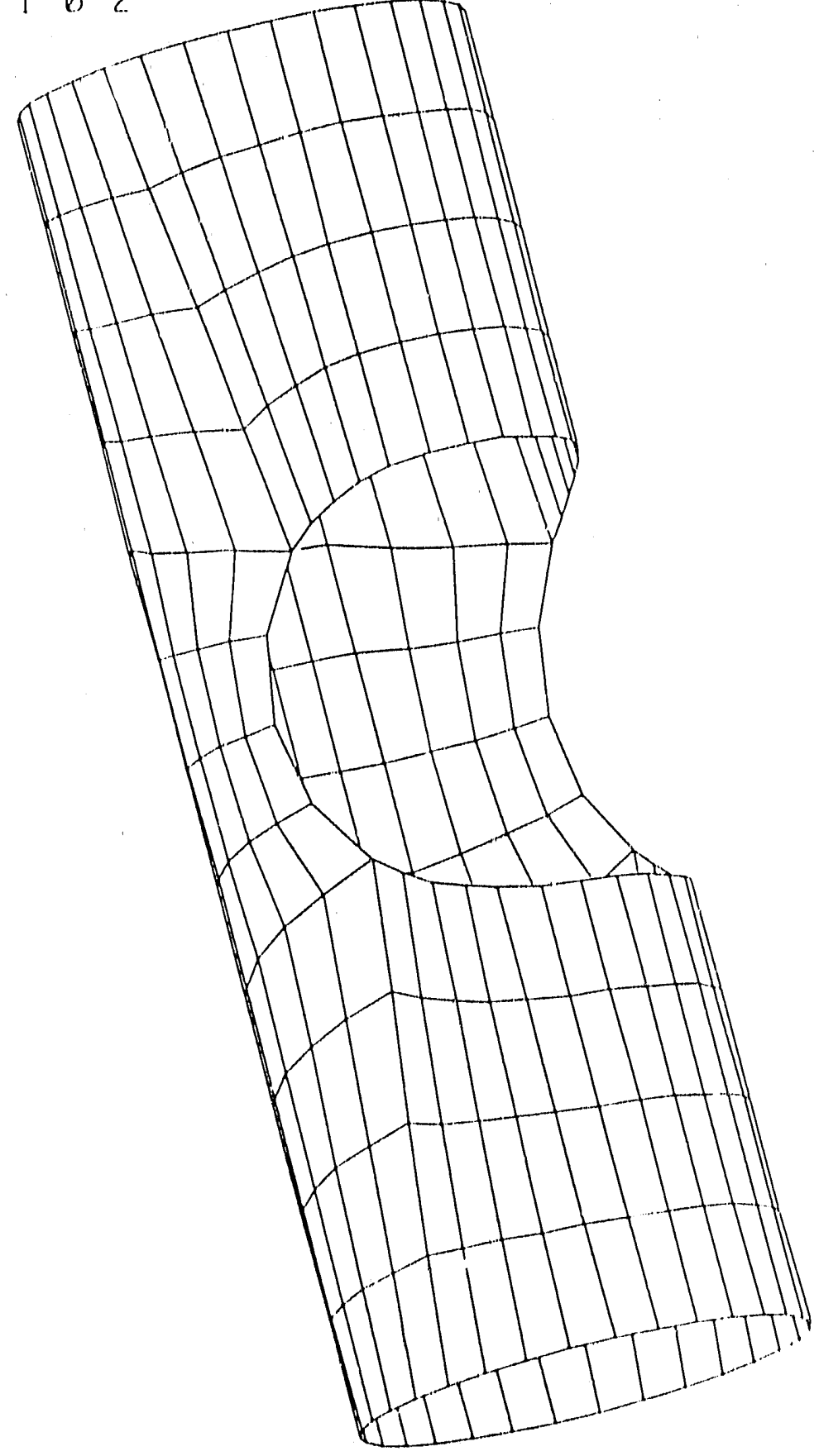


solid cylindrical bar with cylidrical cut out $\sin 3 d$

start $1579913: 1579913: 159913$;

$\begin{array}{lllllllllllllll}-1 & -1 & 0 & 1 & 1 & -1 & -1 & 0 & 1 & 1 & -6 & -2 & 2 & 6\end{array}$

di $12045: 12045$; :

d 302503

sf $-1-5 ;-1-5 ;: c y \theta \theta \theta \theta 01$ ?

sf $-35 ; ;-2-3 ; c y 2000102$ end end

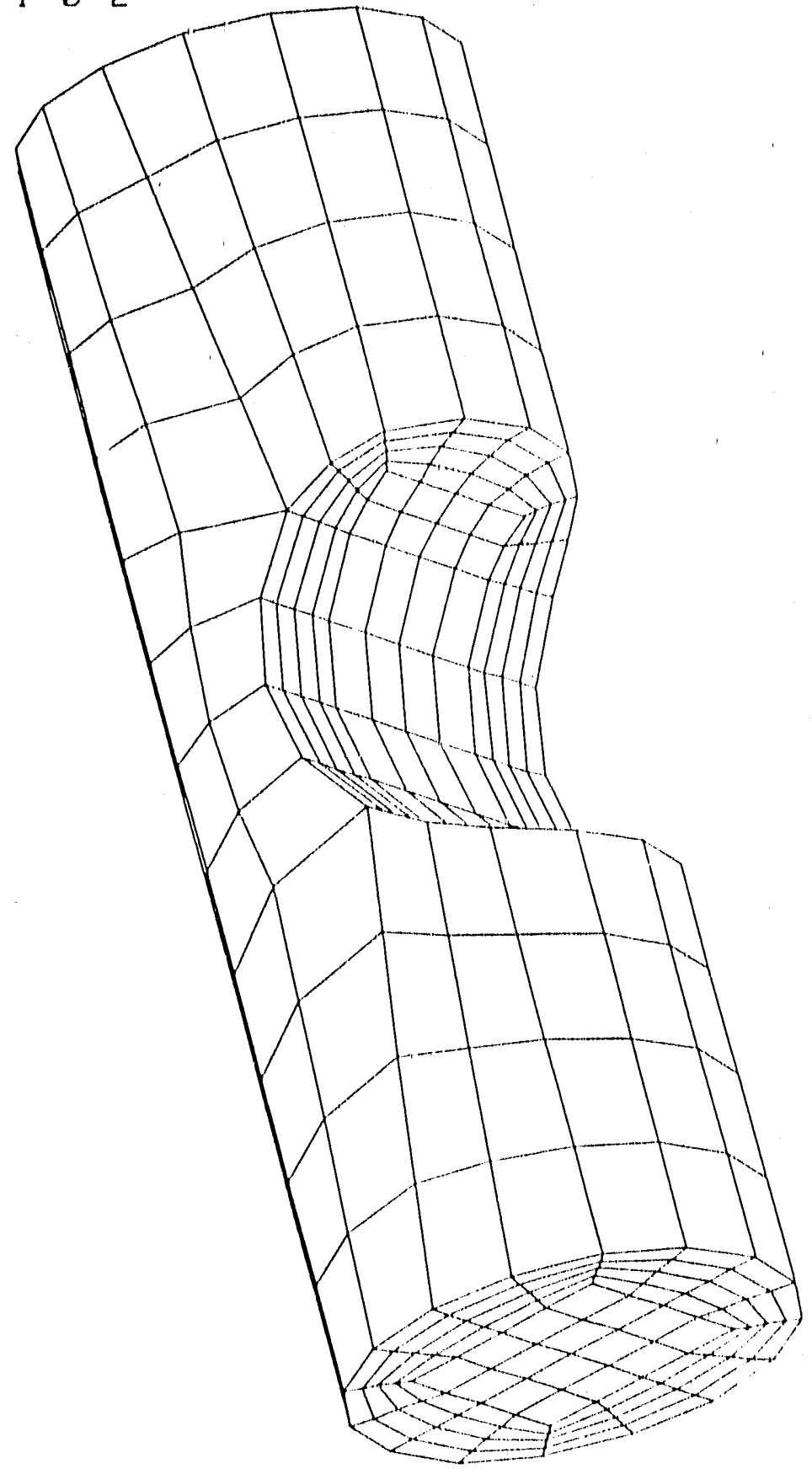


spherical with a hole dn $3 d$

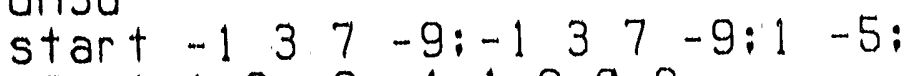

$\begin{array}{llllllllll}-2 & -1 & 1 & 2 & -2 & -1 & 1 & 2 & 0 & 2\end{array}$

sf $1-1-4 ;-1-4: 1-2 ;$ sp 000 ○ 3

d 2.20330

sf $1-2-3:-2-3 ; \quad ; c y \quad \theta \quad 0 \quad 000011$

cpl $0010011+51 J \mathrm{~J}$

end end

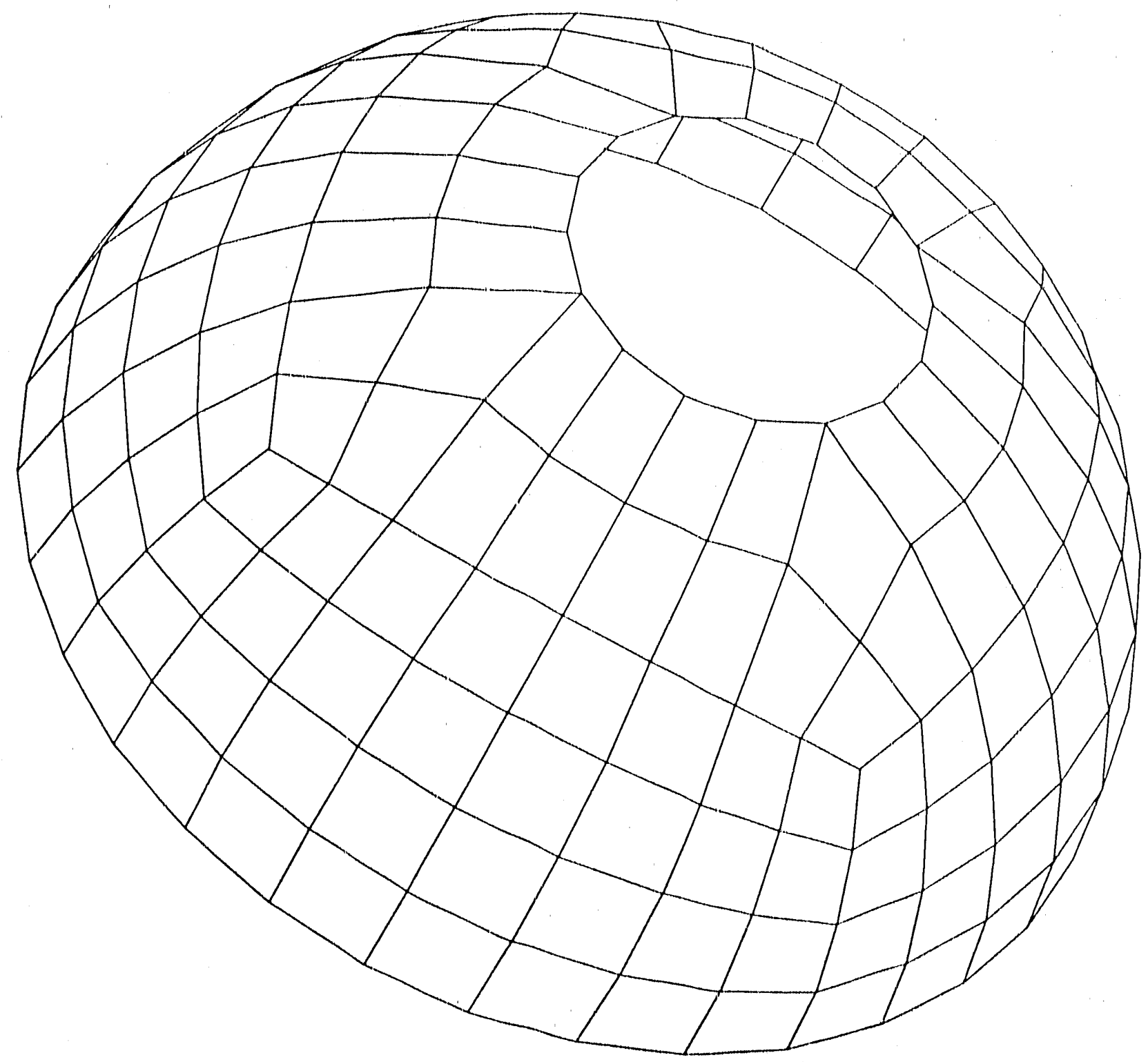


- bracket with hole

dri3d

start 149 . $12: 150$ 13:1 4:

$-1.5-111.5023302$

d 220

pb $040040 x y 05$

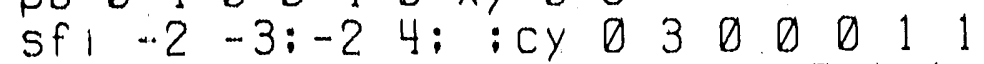

Sf $1-1-4: 34:: c y 03000011.5$

sf 1100130 plan -1.500010

sf 4100430 plan 1.5000100

$\operatorname{cpl} 110130 \mathrm{~J}+13 \mathrm{~J}$

end end

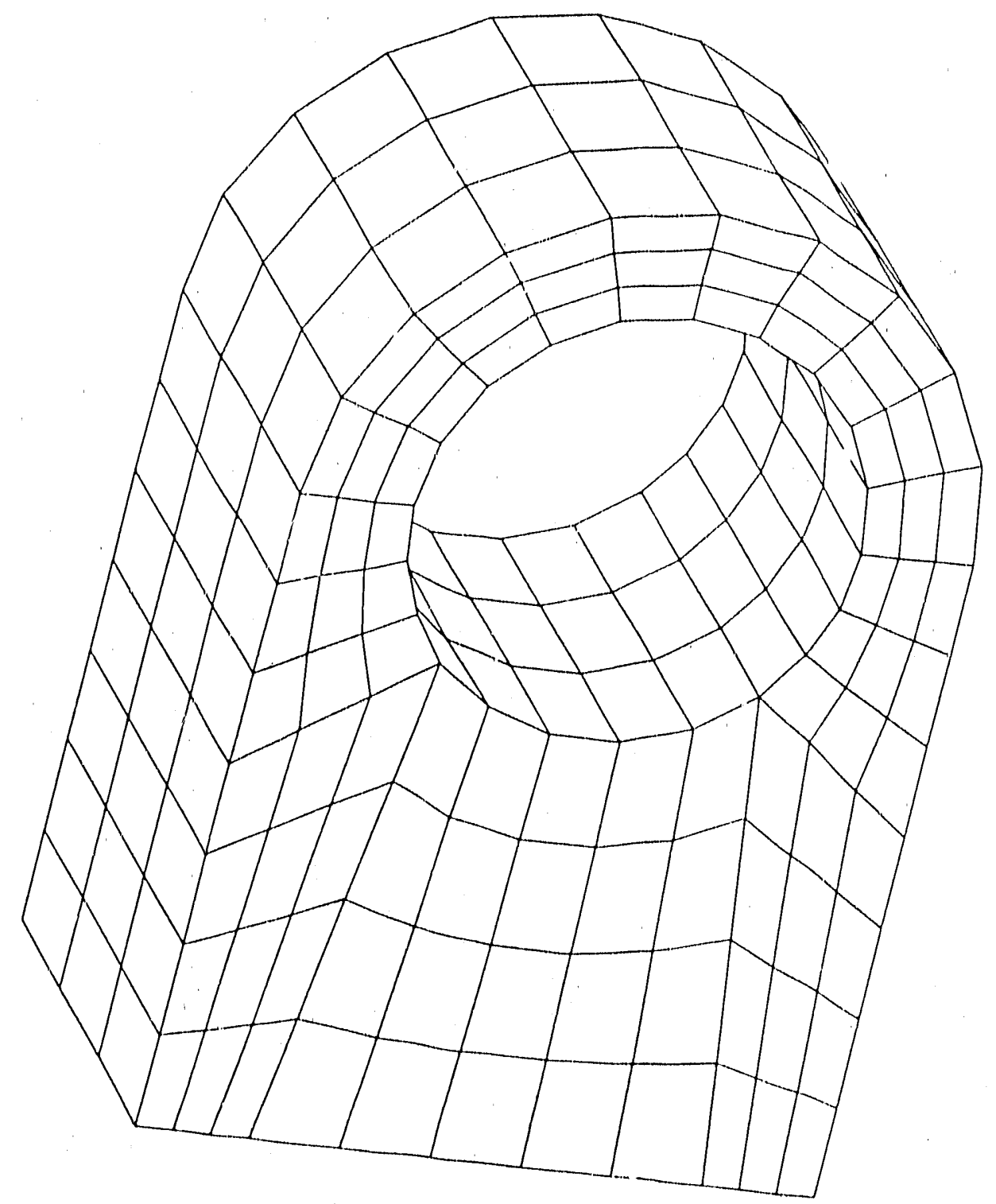


circular flange with hole dn $3 d$

start $1 \quad 5 \quad 9 \quad 13: 1 \quad 37$ 9:1 4:

$\begin{array}{llllllllll}-1 & -1 & 1 & 1 & 10 & 10 & 12 & 12 & 0 & 2\end{array}$

d 2200330

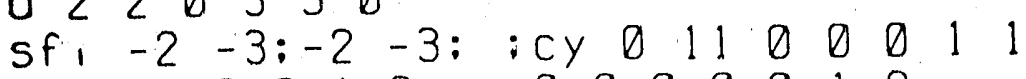

Sf 0 \begin{tabular}{lllllllllllll}
\hline & 0 & 0 & 1 & 0 & $c y$ & 0 & 0 & 0 & 0 & 0 & 1 & 9
\end{tabular}

sf $0 \begin{array}{lllllllllllllll} & 4 & 0 & 0 & 4 & 0 & c y & 0 & 0 & 0 & 0 & 0 & 1 & 13\end{array}$

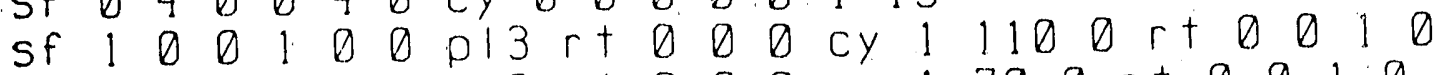

sf 400400 .

$\operatorname{cpl} 100100 \mathrm{~J}+13 \mathrm{~J}$

end end

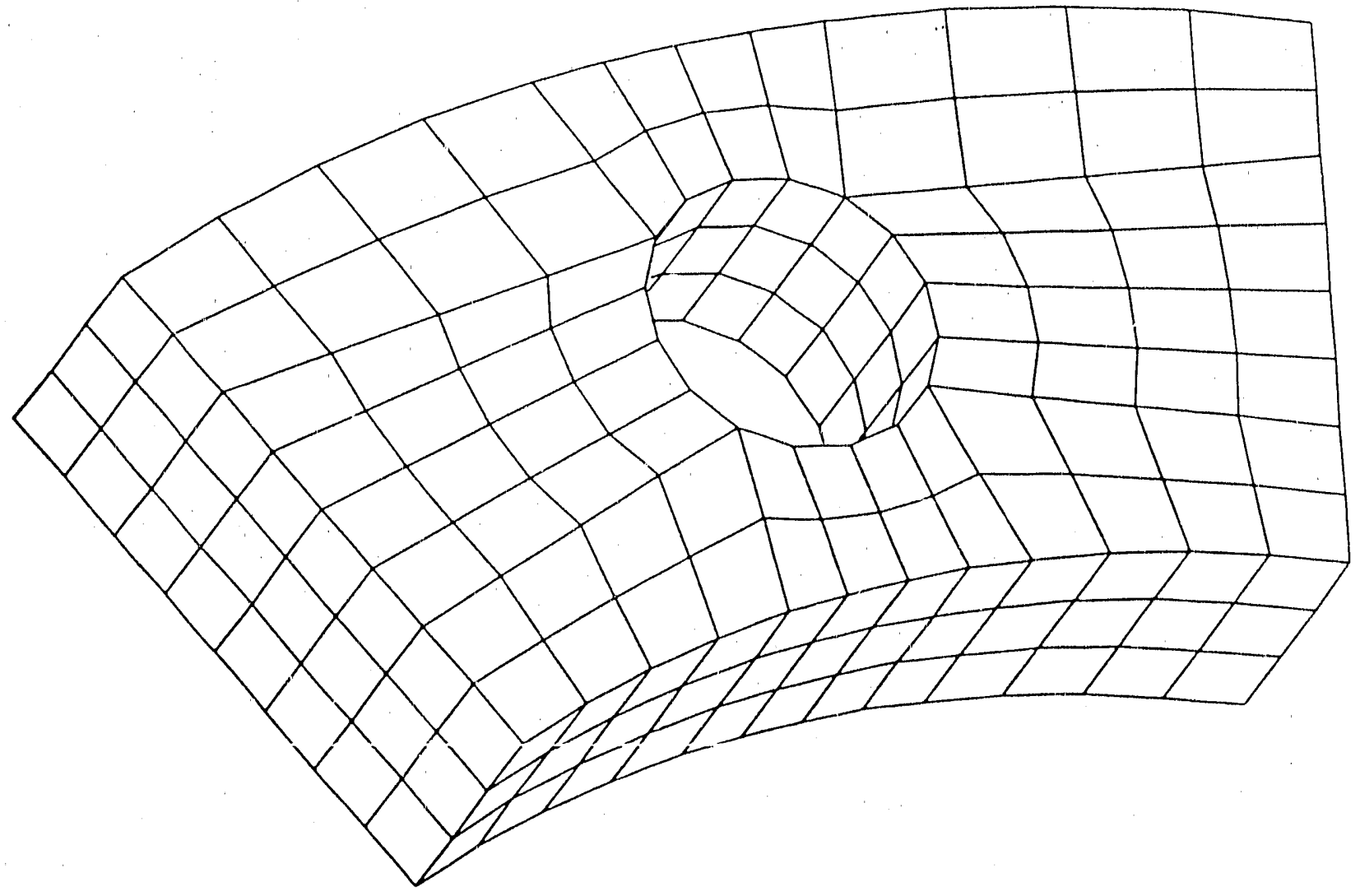


$\therefore \quad$ spherical cap with hole and ${ }^{41}$ trasition region dn $3 d$

start $-1-7 ;-1-7 ; 1$ 4;

$-22-2 \quad 202$

sfi $-1-2 ;-1-2 ;$ isp 000

end

start $171319: 171319:-1$ :

$\begin{array}{lllllllll}-2 & -2 & 2 & 2 & -2 & -2 & 2 & 2 & 2\end{array}$

di $120034: 12034:-1$;

sf $\theta \quad 01001 \operatorname{sp} \theta \quad 0 \quad 02$

d 220330

sf 2200330 cy $\theta 0000001$

end end

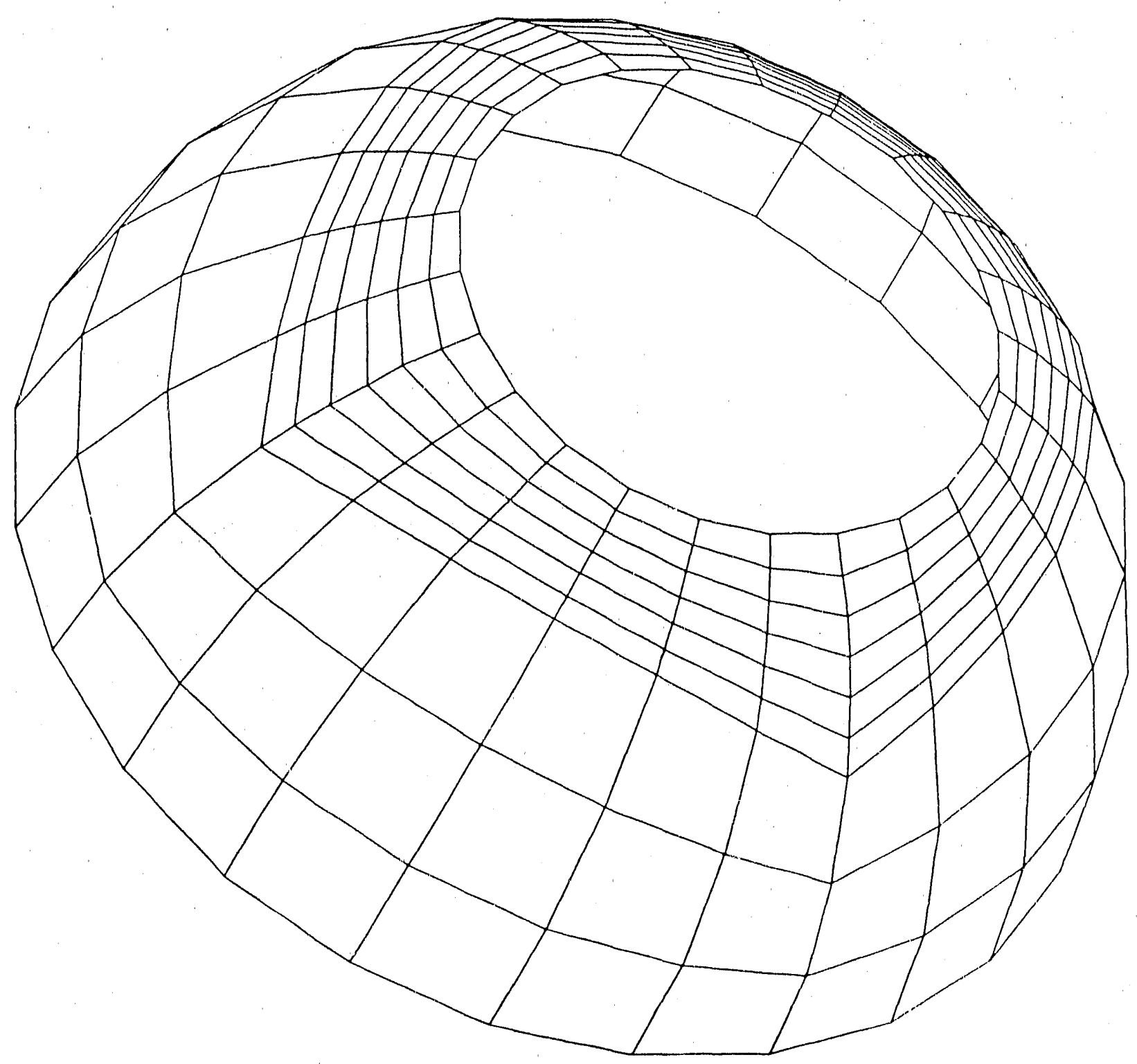


fel decoy by Gregg Mannell dn3d lcd 130111020 parameter r2 22 r1 [\% $2 /$ sqrit(2)] delta 10; mat 11 shell endmat

start - $1.40-41 ;-1-21 ;-1-21$;

$-10095.4100-100100-100100$

mate 1 thic $.0088 \mathrm{~d} 200300$

sfi-1 -3;-1 -2;-1 -2;sp 000100

sf 200300 cy 00010030 end

start -1;1 $42427 ; 142427$;

95.4 [-\%r1] [-\%r1] \%r1 \%r1 [-\%r1] [-\%r1] \%rl \%r1 di ; $12034 ; 12034$;

sfi ;-1 -4;-1 -4;cy 000010030

sf 122123 cy $0 \%$ delta 0100 [\%r2+\%delta] sf 132133 cy 0 [-\%delta] 0100 [\%r2+\%delta] sf 122132 cy $00 \%$ delta 100 [\%r2+\%delta] sf 123133 cy 00 [-\%delta] 100 [\%r2+\%delta] sfi $-1 ; 14 ; 14 ;$ sp 000100 pr 00000011.0000 end end
42

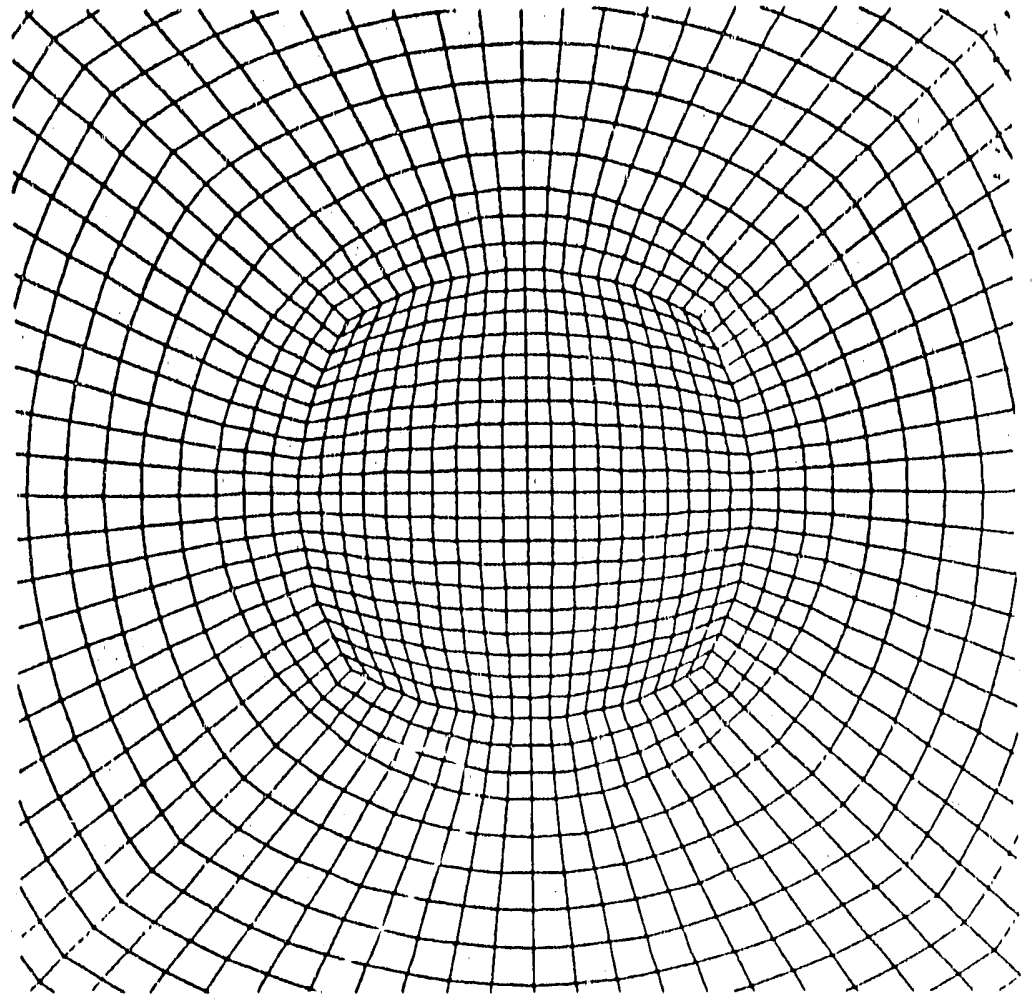

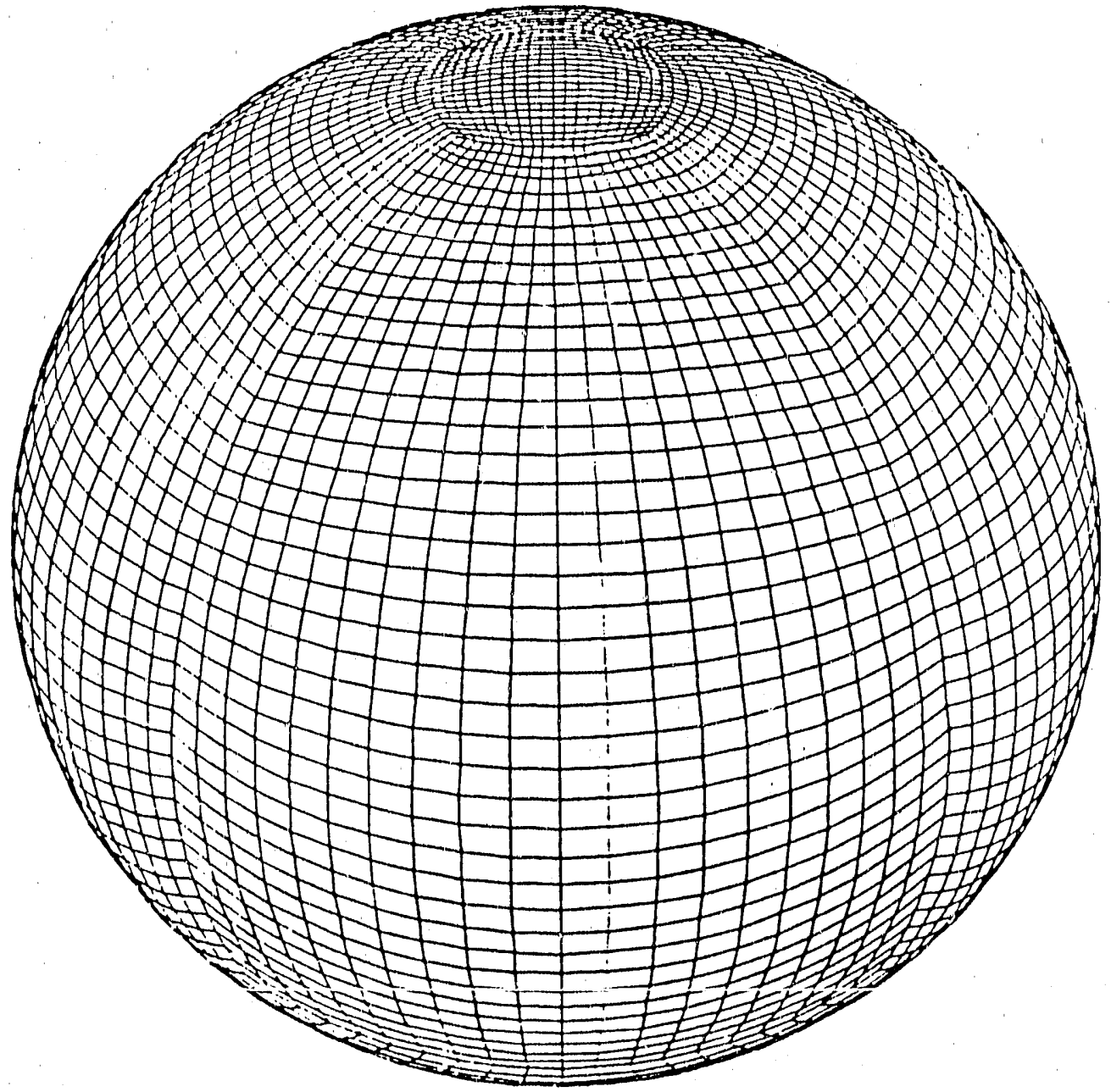


pipe bend with hole dn3d

start $-1 \quad-9: 1 \quad 1523 \quad 37:-1 \quad-9$;

$10150000-2.52 .5$

$\begin{array}{llllllllll} & r & 0 & 2 & 0 & 0 & 2 & 0 & r z & 35:\end{array}$

rr $0 \begin{array}{lllllllll} & 0 & 0 & 0 & 3 & 0 & r z & 55:\end{array}$

rr 04000040 O

sf: $-1-2: \quad:-1 \quad-2: \operatorname{ts} \theta 00000112.5002 .5$

d: 22232

enc

start $191725: 191725:-1$ :

1010.151500002 .5

$m b 2200230 \times 1$

$m b \quad 3 \quad 2 \quad 0 \quad 3 \quad 300 x-1$

rr $0 \begin{array}{llllllll} & 0 & 0 & 0 & 2 & 0 & r z & 35:\end{array}$

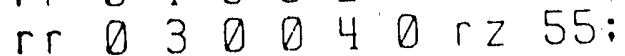

d. $120034: 12034:$ :

d 220330

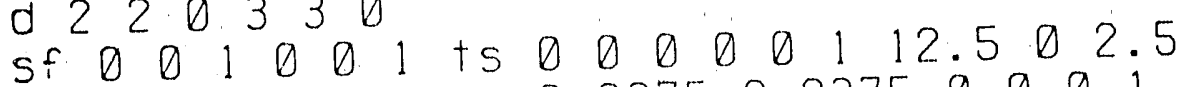

sf $2200.3 \quad 30$ cy $8.8375 \quad 8.83750000$

erid end

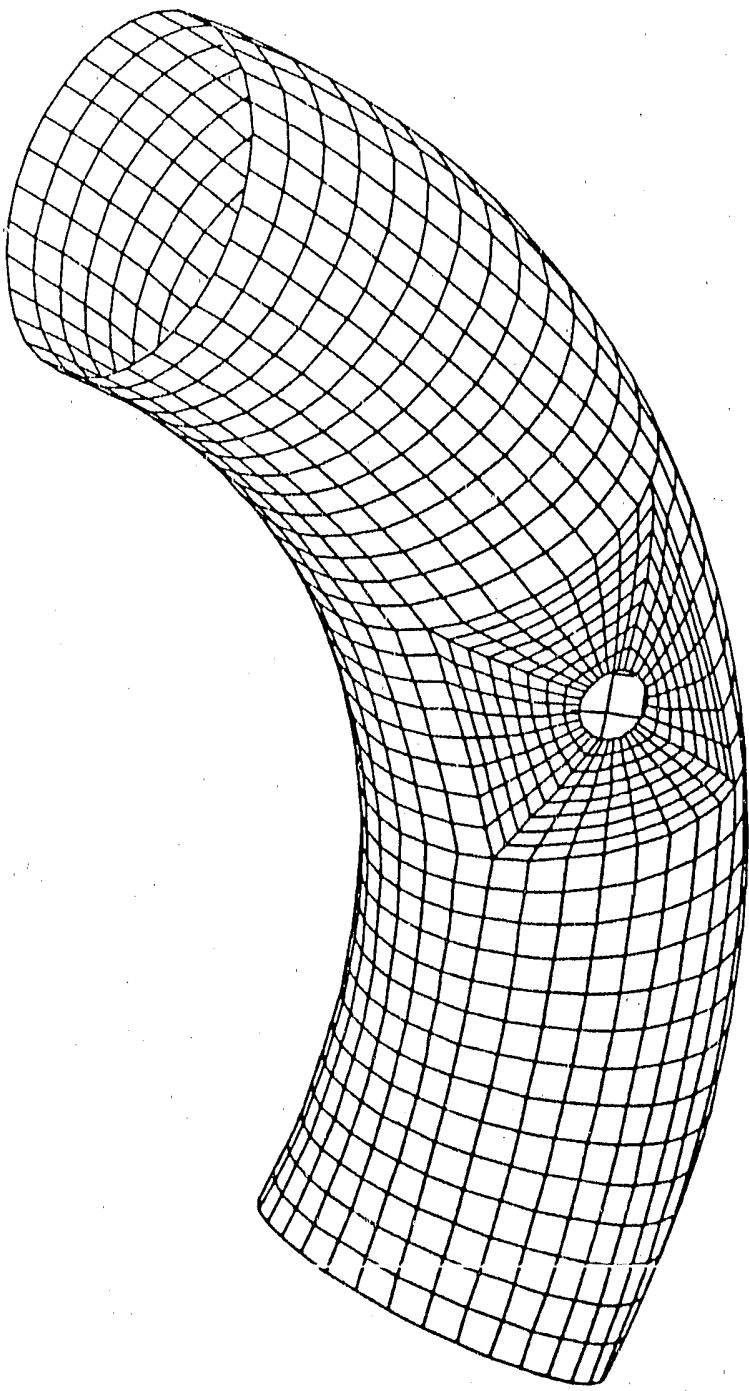


dn $3 d$

sd 1 cy 0

sod 2 cy 00000010

start $-1-10 ;-1-10: 1 \quad 7 \quad 16$ 22:

$\begin{array}{llllllll}-2 & 2 & -2 & 2 & -6 & -2 & 2 & 6\end{array}$

d 0222023

$m b \quad 022023 z 2$

sfi $-1-2 ;-1 \quad-2$; isd 1

sf 022023 sd 2

end

start - 1 - 10; 1 15;-1 -10:

$-2.2 \quad 12-22$

$r r \quad 020020 r \times 45$ :

$m b \quad 0 \quad 1 \quad 0 \quad 0.1 \quad 00 z$

sf $1-1-2 ; ;-1-2 ;$ sd 2

sf 010010 sd 1

end end

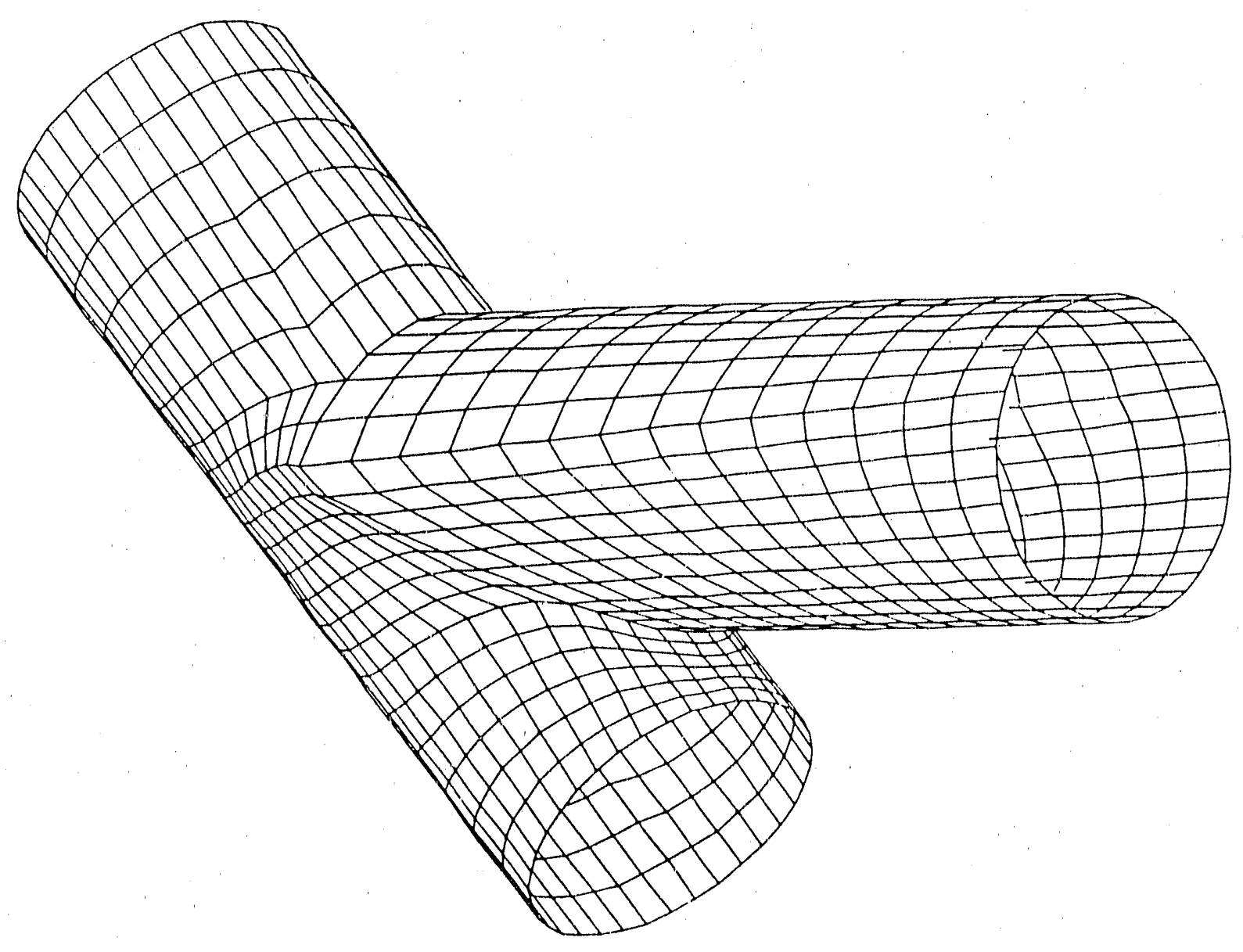


three pipes intersecting

dn $3 d$

so 1 cy 000000012

sd 2 cy $0 \quad 0 \quad 0 \quad 0.866 .52$

sd 3 cy 0 c $000.866-.52$

start $-1-11:-1-11: 1601116$ 21:

$\begin{array}{lllllllll}-2 & 2 & -2 & 2 & -6 & -2 & 0 & 2 & 6\end{array}$

d $0 \begin{array}{llllll}0 & 2 & 2 & 0 & 2 & 4\end{array}$

$p b 02302 \quad 3$ y.

sf 122123 sd $3+1$ sd 3

sf $12 \begin{array}{lllllll}2 & 2 & 2 & 2 & \text { sd } & 3\end{array}$

sf 123124 sd $2+11$ sd 2

sf $12422 \quad 4 \quad$ sd 2

sfi $-1-2:-1-2:$ : sd 1

end

start - 1-11:1 21:-1 6-11:

$-22210-202$

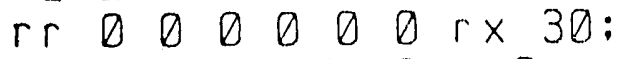

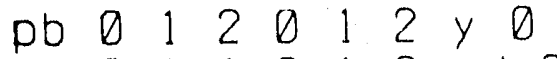

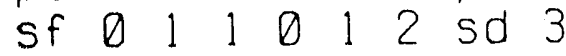

sf $\quad \begin{array}{llllllll}0 & 1 & 2 & 0 & 1 & 3 & \text { sd } & 1\end{array}$

sf $\theta \quad \theta \quad 0 \quad \theta \quad 0 \quad 0$ sd 2

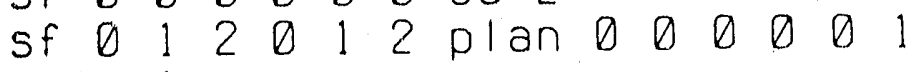

coor I rxy:

Irep 0 1:

end end

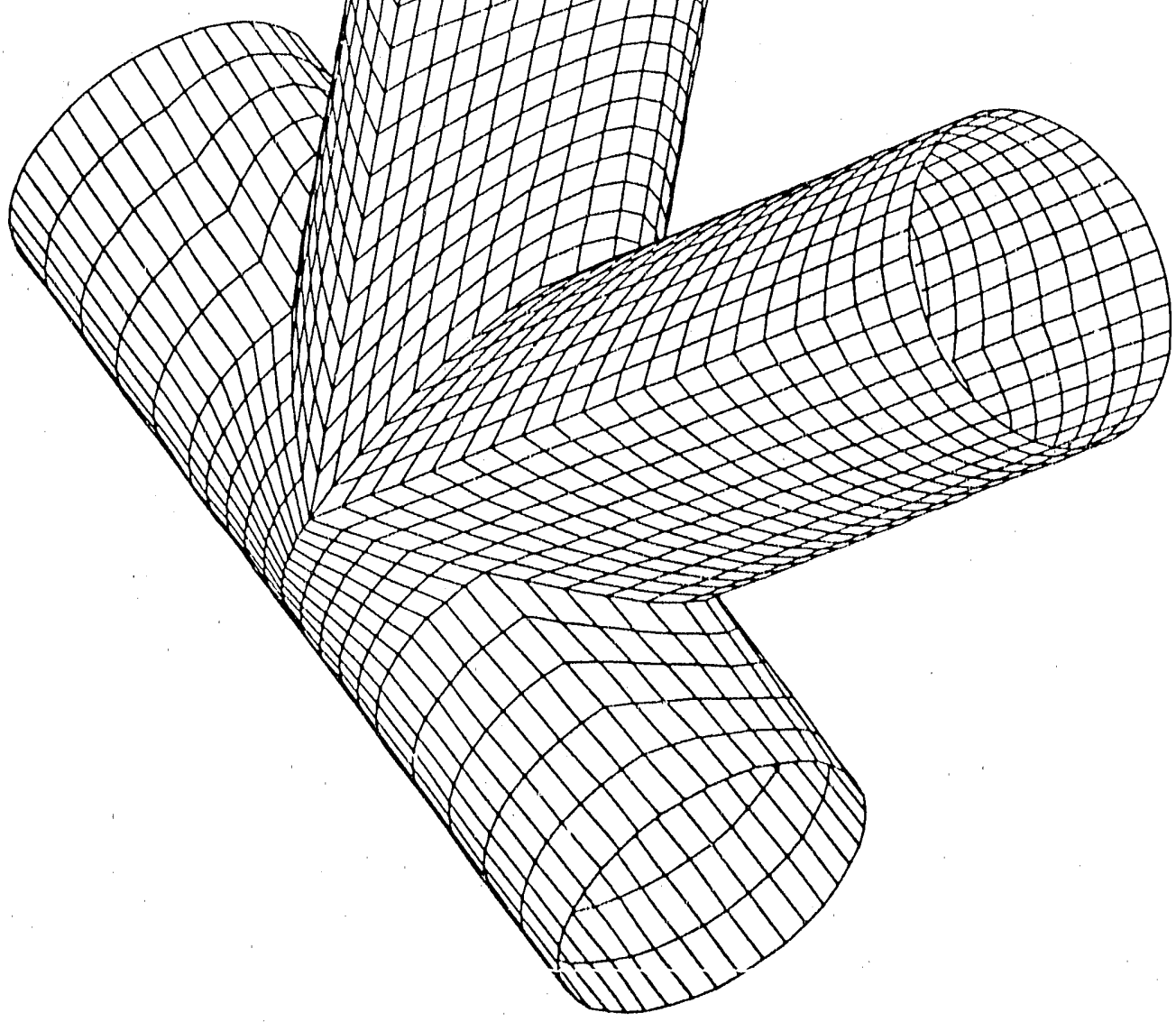


berid sheet with fading bend ${ }^{46}$

dn $3 d$

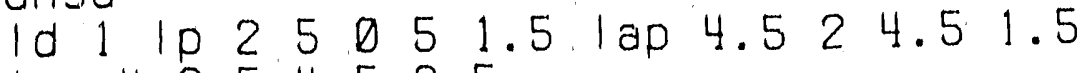

lap $4.2 .5 \quad 4.52 .5$

I ap $4.5 \quad 3 \quad 4.5 \quad 2.5$

lap $5 \quad 3.5 \quad 4.5 \quad 3.5$

Ip 155

start $131:-1: 1 \quad 1631$ :

0 व 5002.55

$\mathrm{pb} 2000200$

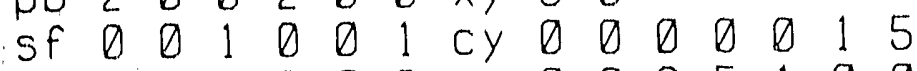

sf $00 \begin{array}{lllllllllllll}2 & 0 & 0 & 2 & \text { er } & 0 & 0 & 2.5 & 1 & 0 & 0 & 4 & 5\end{array}$

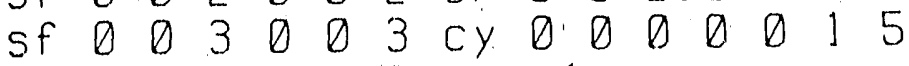

sf $1000100 \operatorname{crz} 1$

end end

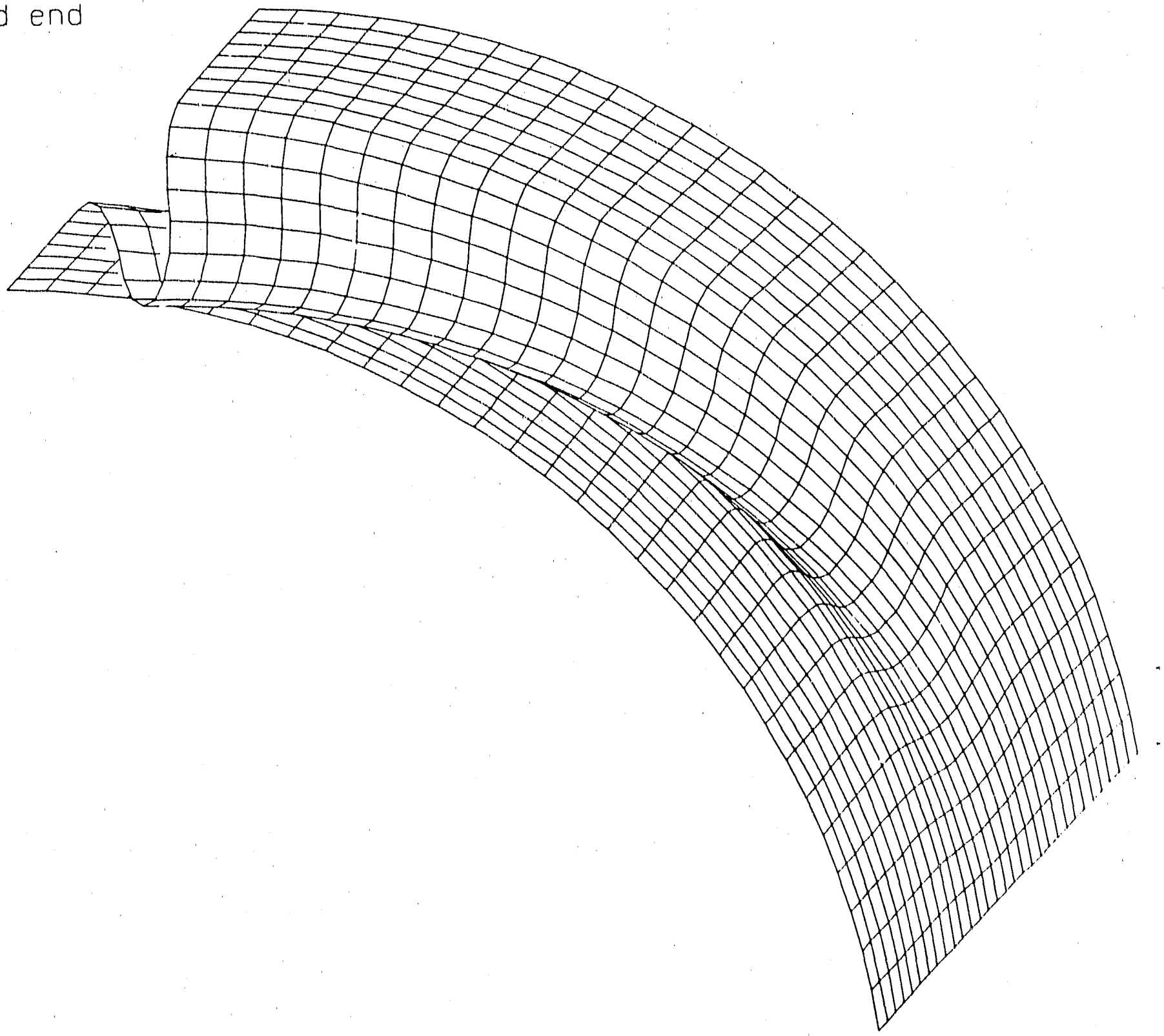


tube within a block

dn $3 d$

start $1511: 11121: 111$;

$.088 .104 .228 \quad 045 \quad 90 \quad 0 \quad .1435$

sf 311322 plan .22800100

sf 3211332 plan 0.2280010

cyli lct 1 ryz: Irep 0 l: end end

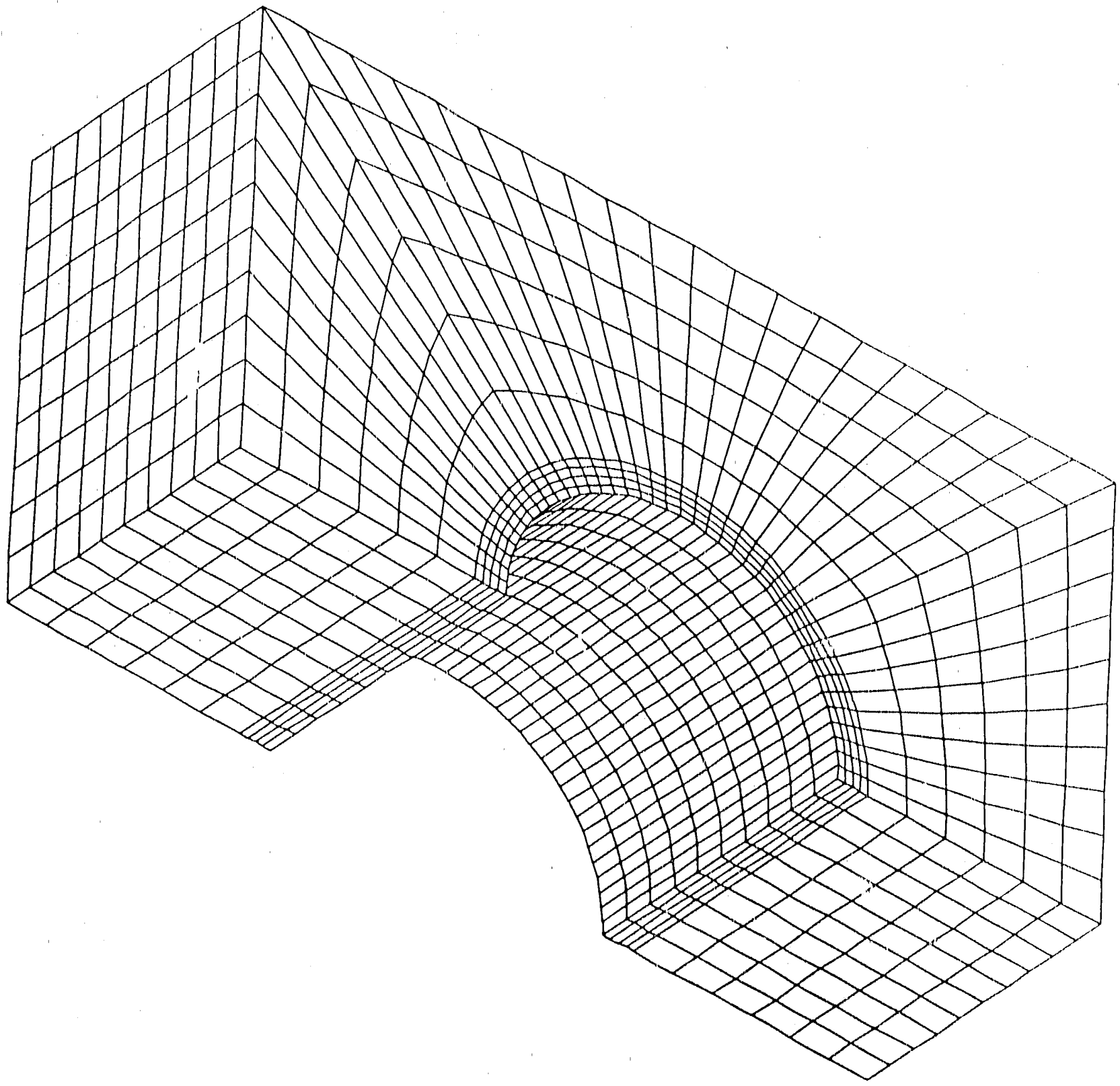


spherical bubble in block with ratio zoning

dn3d

parameter p1 10 p2 5 rs 1.4;

term 1 prti 100 plt .01 itss $1.0 \mathrm{e}-4$

led 12011000001

mat 11 e 3.2 pr 17 ro 2.1

mat 21 e 3.2 pr 17 ro 2.1

endmat

$\cos 11 \mathrm{c} 00 \mathrm{c} 11 \mathrm{c} 22 \mathrm{c} 33$

$\cos 21 \mathrm{c} 00 \mathrm{cl} 1 \mathrm{c} 22 \mathrm{c} 33$

endeos

sd 1 sp 00002

sd 2 sp 00001.5

plane $200000-1.01$ symim

$000-100.01 \mathrm{symm}$

start

$1[1+\% \mathrm{p} 1][1+\% \mathrm{p} 1+\% \mathrm{p} 2]$

$1[1+\% \mathrm{p} 1][1+\% \mathrm{p} 1+\% \mathrm{p} 2]$

$1[1+\% \mathrm{p} 1][1+\% \mathrm{p} 1+\% \mathrm{p} 2]$;

044044044

do22033

d 202303

d 220330

d 000222

res 211322 i \%rs

res $121232 \mathrm{j} \%$ rs

res $112223 \mathrm{k} \%$ rs

mate 1

pr 11322311000

sf $112222 \mathrm{sd} 1$

sf $121222 \mathrm{sd} 1$

sf $211222 \mathrm{sd} 1$

lct 1 ; ryz; lrep 01 ;

end

end
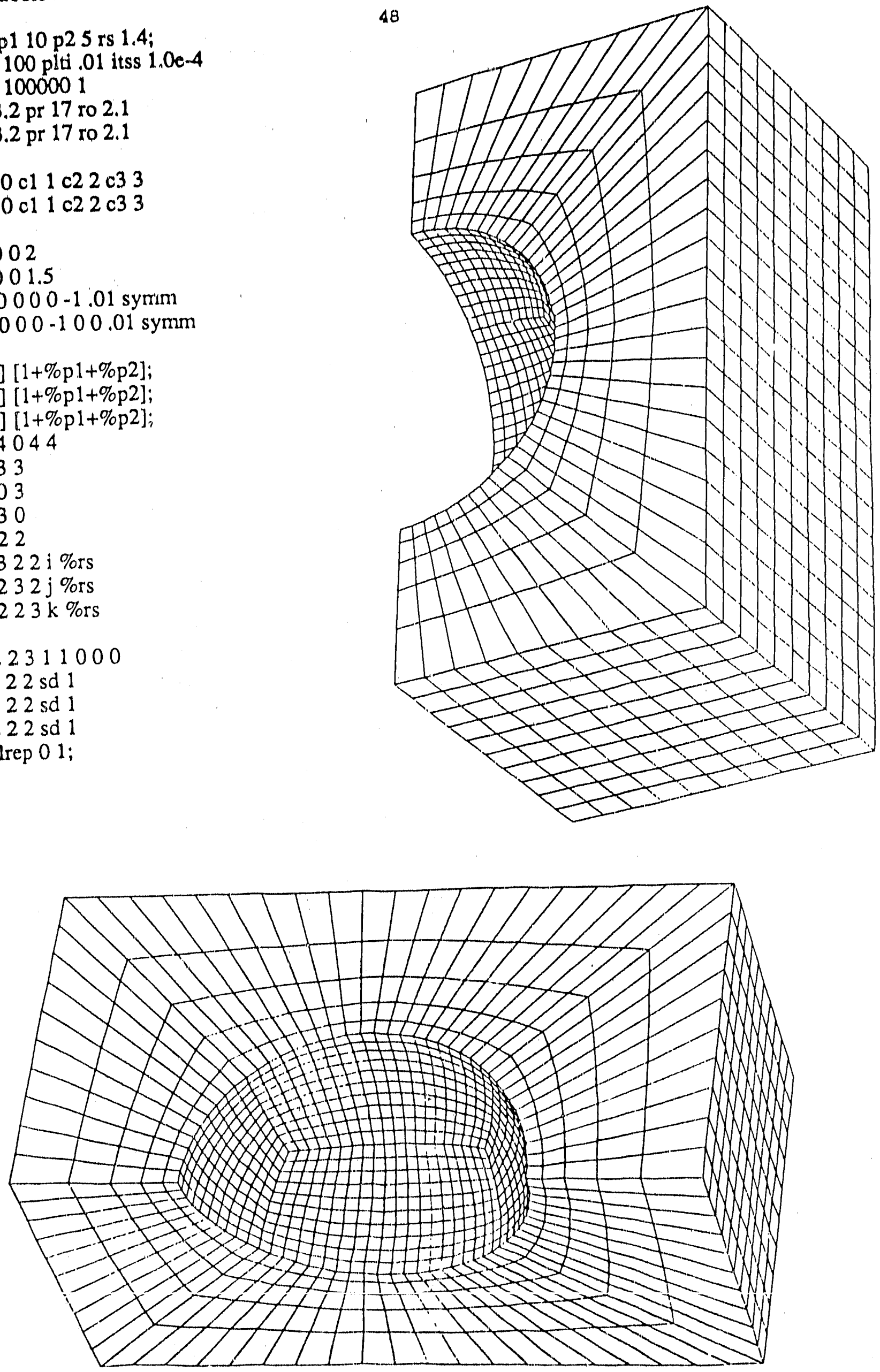
exploded view of a diaphram made of shell elements start

132325 :

132325 :

$120-30410$ :

$-.7-.7 .7 .7$

$-.7-.7 .7 .7$

0.0501022 .3

di $12045: 120.45: 17$ :

di $2 \quad 3: 2 \quad 3: 12007$ :

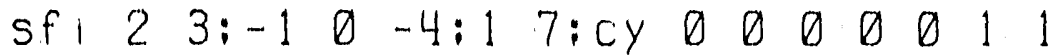

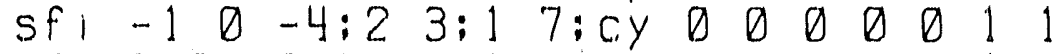

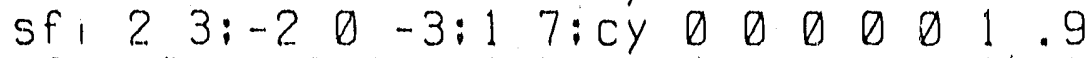

sf: $-2 \quad 0 \quad-3: 2 \quad 3: 1 \quad 7: c y \quad 0 \quad 000001 \% 9$

end

end

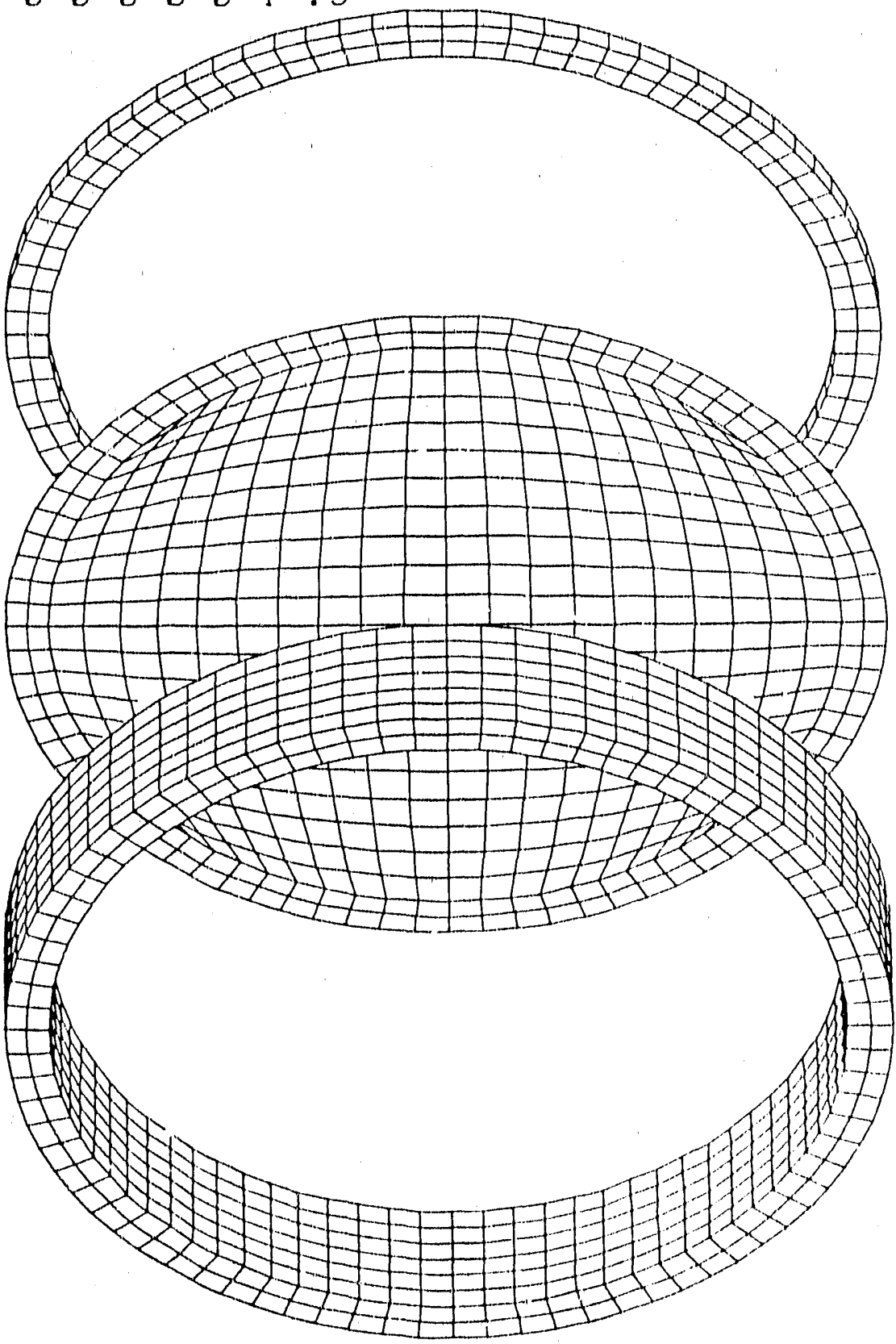


channel

nk3d

mat 11

e 1 .

pr 1 .

shell

endmat

start

$153:-1-10 ;-10-2-11$ :

$\begin{array}{ll}1 & 2.650 .500 .25 .5\end{array}$

$p b=0 \begin{array}{llllllll}4 & 4 & 0 & 1 & 4 & y & .1\end{array}$

pb $0 \begin{array}{llllllll} & 2 & 4 & 0 & 2 & 4 & y & .4\end{array}$

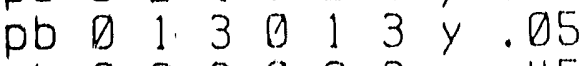

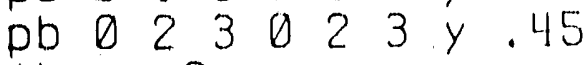

thic . 2

mate 1

end

start

$153:-10-2:-1-10$;

02.6500 .50 .25

$p b \quad 0125012 y$

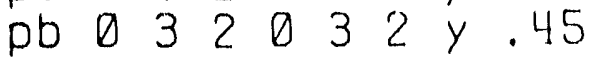

thic 4

mate 1

end

end

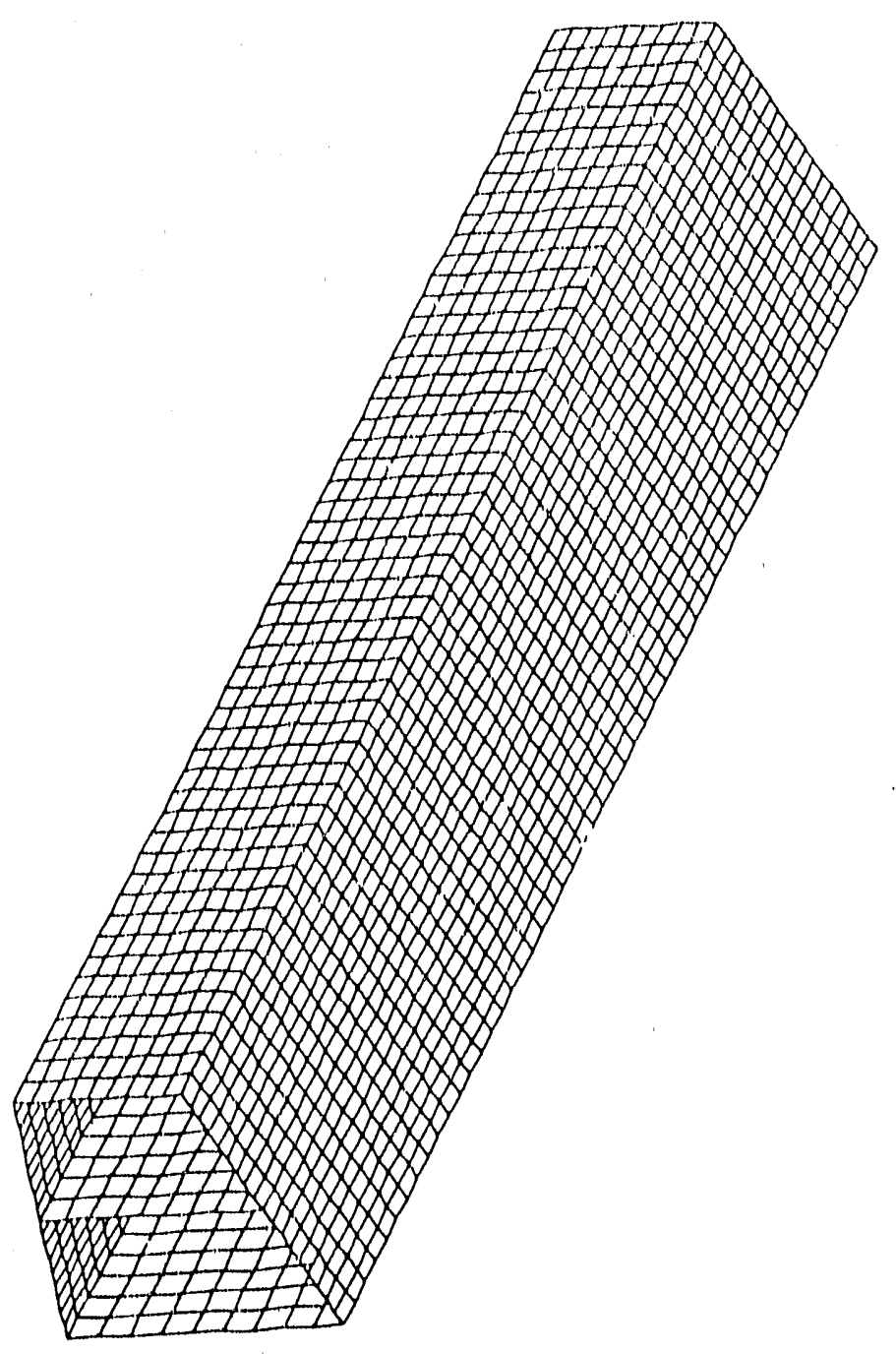


tiger cask by Ous Aramayo dn3d87

c delta - offset for interior cylinder surface of part 1

c rad - radlus of interlor cylinder surface

c th - gap between cylinder container and other parts

$c$ sth - shell thickness of cyllinder

c bth - box thickness

parameter delta 2 rad [\%deltat6] th .125 sth .25 bth 1;

si $1 \mathrm{sV} ; \mathrm{c}$ top of box and top of foam

si 2 sv; $c$ botlom of box and bottom of foam

si 3 sv; $c$ left of box and foam

si 4 sV; $c$ right of box and foam

si' $5 \mathrm{sv}$; $c$ front of box and foam

si $6 \mathrm{sv}$; $\mathrm{c}$ back of box and foam

si $7 \mathrm{sv}$; $c$ between two halves of box

si $8 \mathrm{sv}$; $\mathrm{c}$ between two halves of foam

si $9 \mathrm{sv}$; $\mathrm{c}$ front cap and front foam

si 10 sv; $c$ back cap and back foam

si $11 \mathrm{sv}$; $\mathrm{c}$ between cylinder and foam

si $12 \mathrm{sv}$; $\mathrm{c}$ front cap and contents

si 13 sv; c back cap and contents

si 14 sv; $c$ between cylinder and contents

sd 1 ci; 0000018

sd 2 sp $00[14+\%$ th $][8-\%$ th $]$

sd $3 \mathrm{sp} 00$ [86-\%(h)] [8-\%(h)]

sd 4 cy 000001 [8-\%th]

sd 5 sp 00148

sd 6 cy $000001[8+\%$ th]

mat 13 shell $c$ contalner

mat $23 \mathrm{c}$ contents

mat $33 \mathrm{c}$ wood

mat 43 endmat $c$ foam

velocity 00 [sqrt $(386.4 * 360)$ ]

plane 100100001.001 ston

start -1 -7;-1 -7;1 45;-8 8 -8 8694 c cylinder
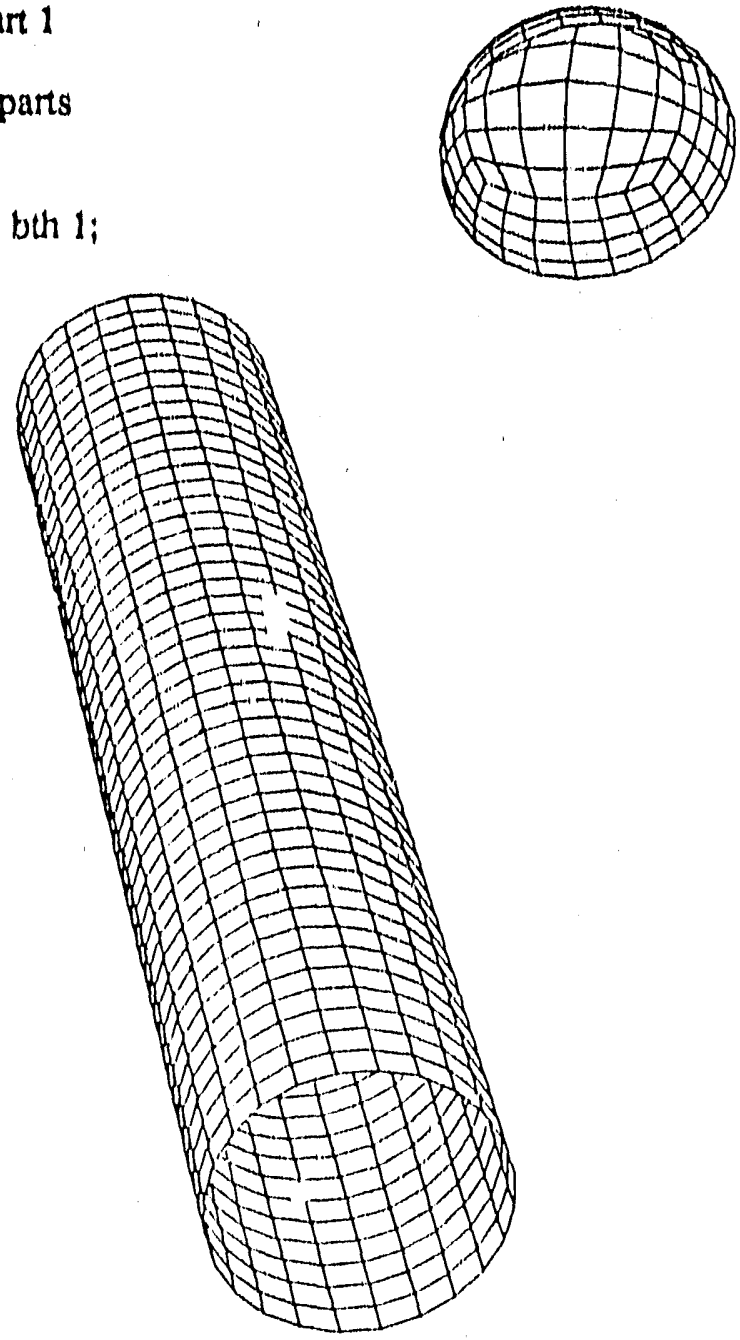

sil- - $1-2 ;-1-2 ; ; 11 \mathrm{~m} 0050$ sil $+-1-2 ;-1-2 ; ; 14 \mathrm{~m} 0050$

thic \%osth mate 1 sfi $-1-2 ;-1-2 ;$; sd 1 end

start $151115 ; 1511 \quad 15 ;-1 ;-8-888$-8 -8880 c cylinder cap

sf 220320 cy $0 \%$ delta $0001 \% \mathrm{rad}$

sf 230330 cy 0 [-\%delta] $0001 \%$ rad

sf $220230 \mathrm{cy} \%$ delta $00001 \% \mathrm{rad}$

sf 320330 cy [-\%delta] $00001 \% \mathrm{rad}$

mate 1 thic \%sth di $12034 ; 12034 ; i$

sf $100100 \mathrm{sd} 1 \mathrm{sf} 400400 \mathrm{sd} 1$

sf 010010 sd 1 sf 040040 sd 1 sf 000000 sd 5

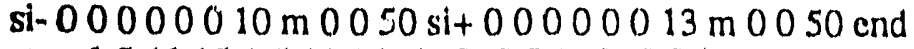

start $151115 ; 1511$ 15;-1;-8 -8 8 8 -8 -8 880 c cylinder cap

sf 220320 cy $0 \%$ delta $0001 \%$ rad

sf 230330 cy 0 [-\%delta] $0001 \% \mathrm{rad}$

sf 220230 cy \%delta $00001 \%$ rad

sf 320330 cy [-\%della] $00001 \%$ rad

mate 1 thic \%sth di $12034: 12034: i$

sf 100100 sd 1 sf 400400 sd 1

sf 010010 sd 1 sf 040040 sd 1 sf 000000 sd 5

si. $0000009 \mathrm{~m} 0050 \mathrm{si}+00000012 \mathrm{m0050}$

lat 1; ixy mz 100;1rep 1;end

start $136911: 136911: 135151719 ;$ c material in cylinder

-8 -.8 $0888-8-808810.510 .5[14+\%$ th $][86-\%$ th $] 89.589 .5$

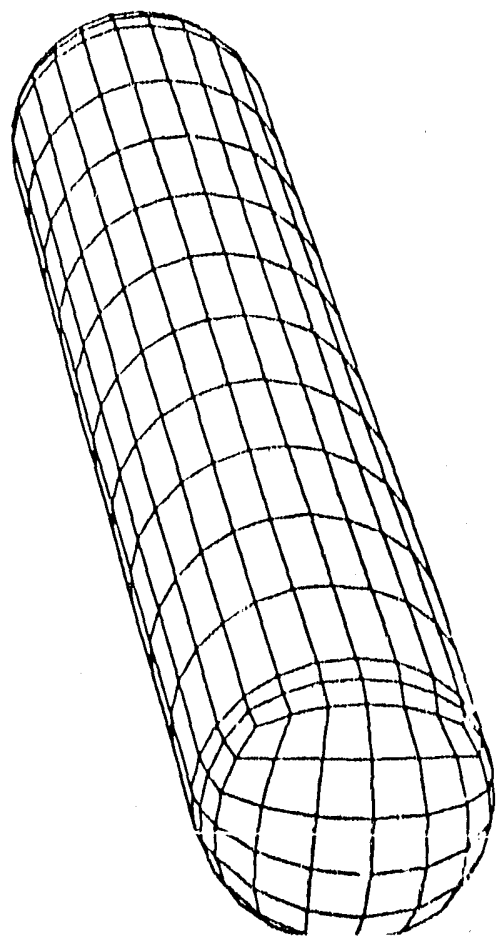


di $12045: 12045: 13046 ;$ dl 2 d:12045:12056; di $12045 ; 24 ; 12056 ;$ mate 2 sil $-1-5 ;-1-5 ; 34 ; 14 \mathrm{~s}$ 52 s1 $22644612 \mathrm{~s}$ sil $-1-5 ;-1-5 ; 45 ; 12 \mathrm{~s}$ si 22144113 s sil $-1-5 ;-1-5 ; 23 ; 13$ s sfi - $-1-5 ;-1-5 ; 34 ;$ sd 4 sfi $-1-5 ;-1-5 ; 13 ;$ sd 2 sfi $-1-5 ;-1-5 ; 46 ;$ sd 3 sf 221441 sd 2 sf 226446 sd 3 pb 222425 y -3 pb 242445 y 3 pb $222245 \times-3$ pb $422445 \times 3$ end start $156 ; 121314 ; 124041 ; \mathrm{d}$ upper half of box $09[9+\%$ bth $][-12-\%$ bth $]-1212[12+\%$ bth] [-\%bth] $0100[100+\%$ bth $]$ mate $3 \mathrm{~d} 122233 \mathrm{sw} 0040041$

si $2222331 \mathrm{~m}$ si $1222233 \mathrm{~m} \mathrm{sil} 1322334 \mathrm{~m}$ si $1232335 \mathrm{~m}$ si $1222326 \mathrm{~m} \mathrm{sil} 1001007 \mathrm{~m}$ end start $156 ; 121314 ; 124041$; c lower half of box 09 [9+\%bth] [-12\%obth] $-1212[12+\%$ hth] [-\%bth] $0100[100+\%$ bth $]$ si $2222332 \mathrm{msl} 1222233 \mathrm{msl} 1322334 \mathrm{~m}$ si $1232335 \mathrm{msl} 1222326 \mathrm{msl} 1001007 \mathrm{~s}$ d 122233 sw 0040041 mate 3 lat liryzilrep liend start 145;1289:131315:099-12-1212120694100 c upper foam di $23 ; 12034 ; 13 ;$ d 122233 mate 4

pb $220230 \times 8$ pb 120220 y -8 pb 130230 y 8 sfi $12 ;-2-3 ; 23 ;$ sd 6 sf 222233 sd 6 si $3003001 \mathrm{~s}$ si $0100103 \mathrm{ssi} 0400404 \mathrm{ssl} 0040045 \mathrm{~s}$ si $0010016 \mathrm{~s} \mathrm{sil} 1001008 \mathrm{msi} 1232339 \mathrm{~s}$ si $12223210 \mathrm{~s}$ si $22223311 \mathrm{~s}$ sil $12:-2-3 ; 23 ; 11 \mathrm{~s}$ end start $145 ; 1289 ; 131315 ; 099-12-1212120694100 \mathrm{c}$ lowwer foam dl $23: 12034: 13:$ d 122233 mate 4

pb $220230 \times 8$ pb 120220 y -8 pb 130230 y 8 sfi $12 ;-2 \cdot 3 ; 2.3 ;$ sd 6 sf $222233 \mathrm{sd} 6 \mathrm{sl} 1001002 \mathrm{ssi} 0100103 \mathrm{~s}$ si $0400404 \mathrm{ssi} 0040045 \mathrm{ssi} 0010016 \mathrm{~s}$ si $1001008 \mathrm{~s}$ si $1232339 \mathrm{~s}$ si $12223210 \mathrm{~s}$ si $22223311 \mathrm{~s}$ sil $12 ;-2-3 ; 2.3 ; 11 \mathrm{~s}$ lat 1 ;ryz; lrep 1 ; end bptol $68-1$ bptol 12.35 bptol 13.35 end $c$ use stp .001 to merge
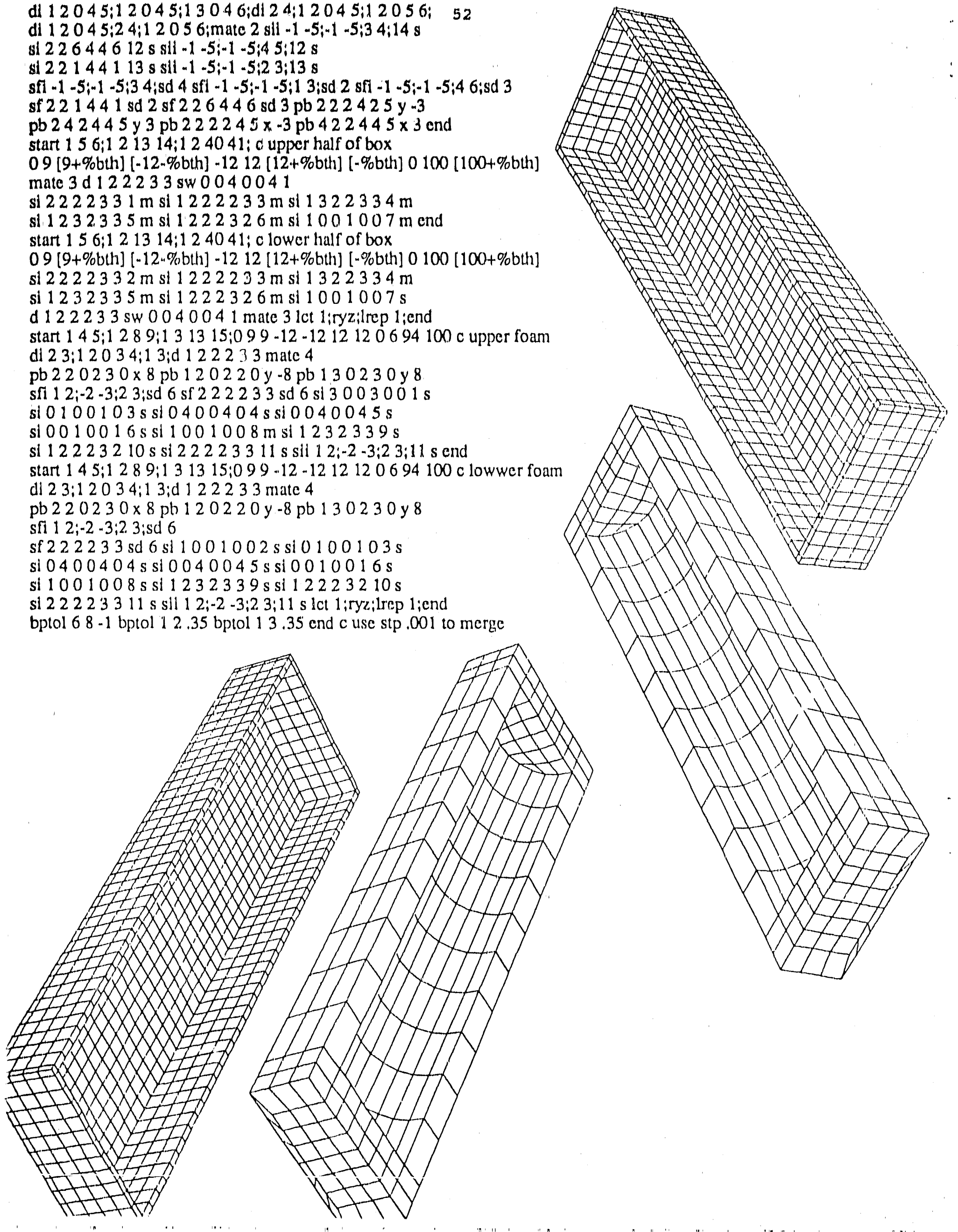
moebius strip

start $111 ; 12 ; 1181 ;-11-.05 .0500$

edit

cyli

$y=y+k$

cart

$x=x+4$

ycyli

$x=k * 2$

end

end

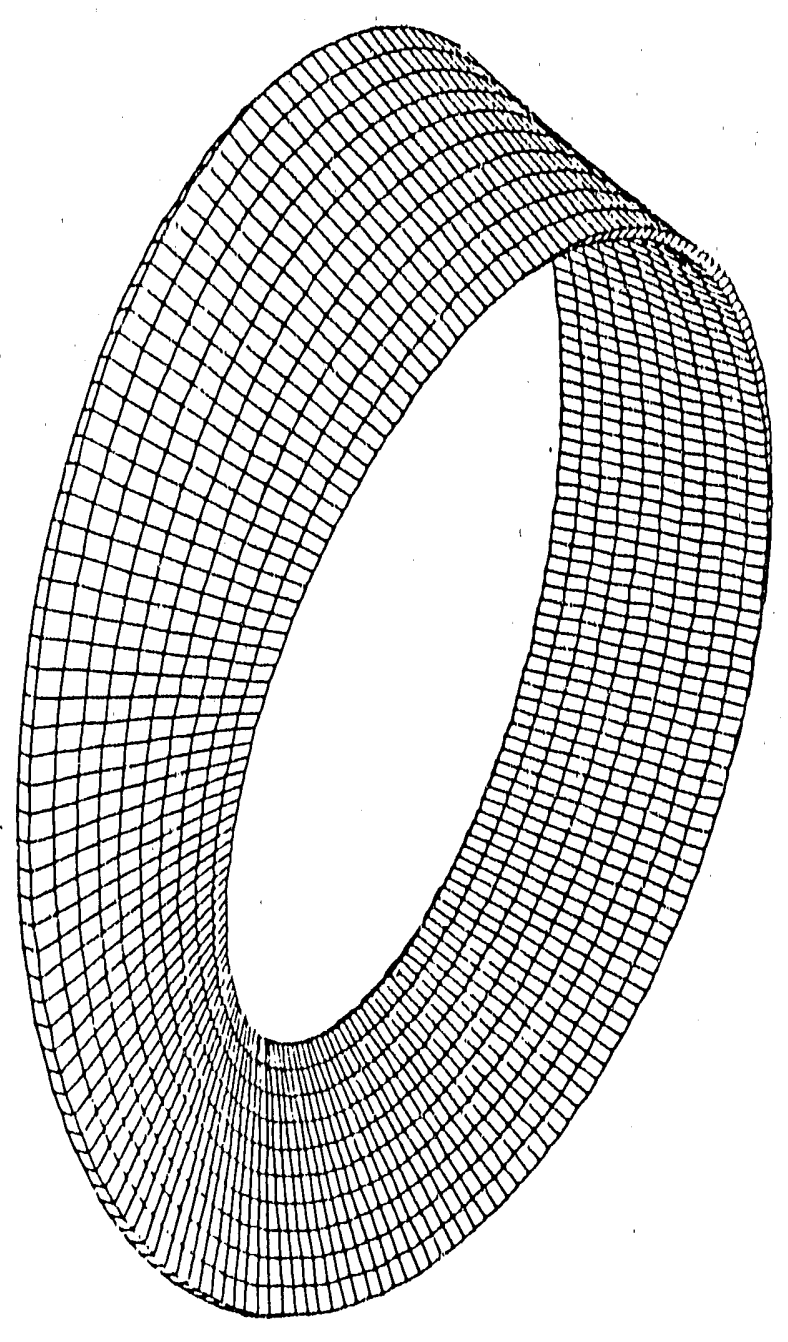




\section{dn3d}

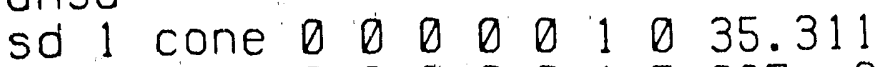

sd 2 cone $0 \quad 0 \quad 0 \quad 0001.5 .667-35.311$

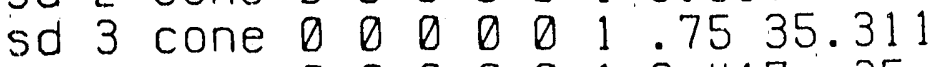

sd 4 cone $00000016.417-35.311$

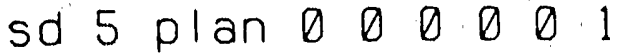

sd 6 plan $0.0 .8 \quad 001$

start

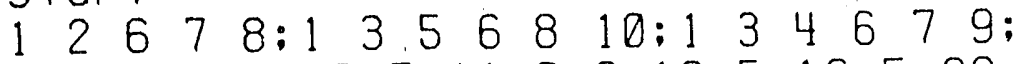

$4.25 .51010 .51108 \quad 13.516 .522 \quad 30023568$ cyl 1

di 2 3:1 30 0 4 6: :

d) 2 3: : 13 o 4 6:

di $34: 1200$ 6: :

di $34: 25: 1205$ 6:

di $12: 12005$ :

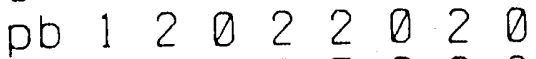

$\begin{array}{llllllllll} & \mathrm{pb} & 1 & 5 & 0 & 2 & 5 & 0 & 2 & 30\end{array}$

$m b 2302302-1.3+j 121.3$

sf 10101102 sd $2+k 4$ sd 1

sf 201202 sd $4+k 4$ sd 3

sf 00101001 sd 5

sf $\theta \begin{array}{llllllll} & 0 & 0 & 0 & 6 & \text { sd } & 6\end{array}$

$c p 120150 \mathrm{~J}$

end

end

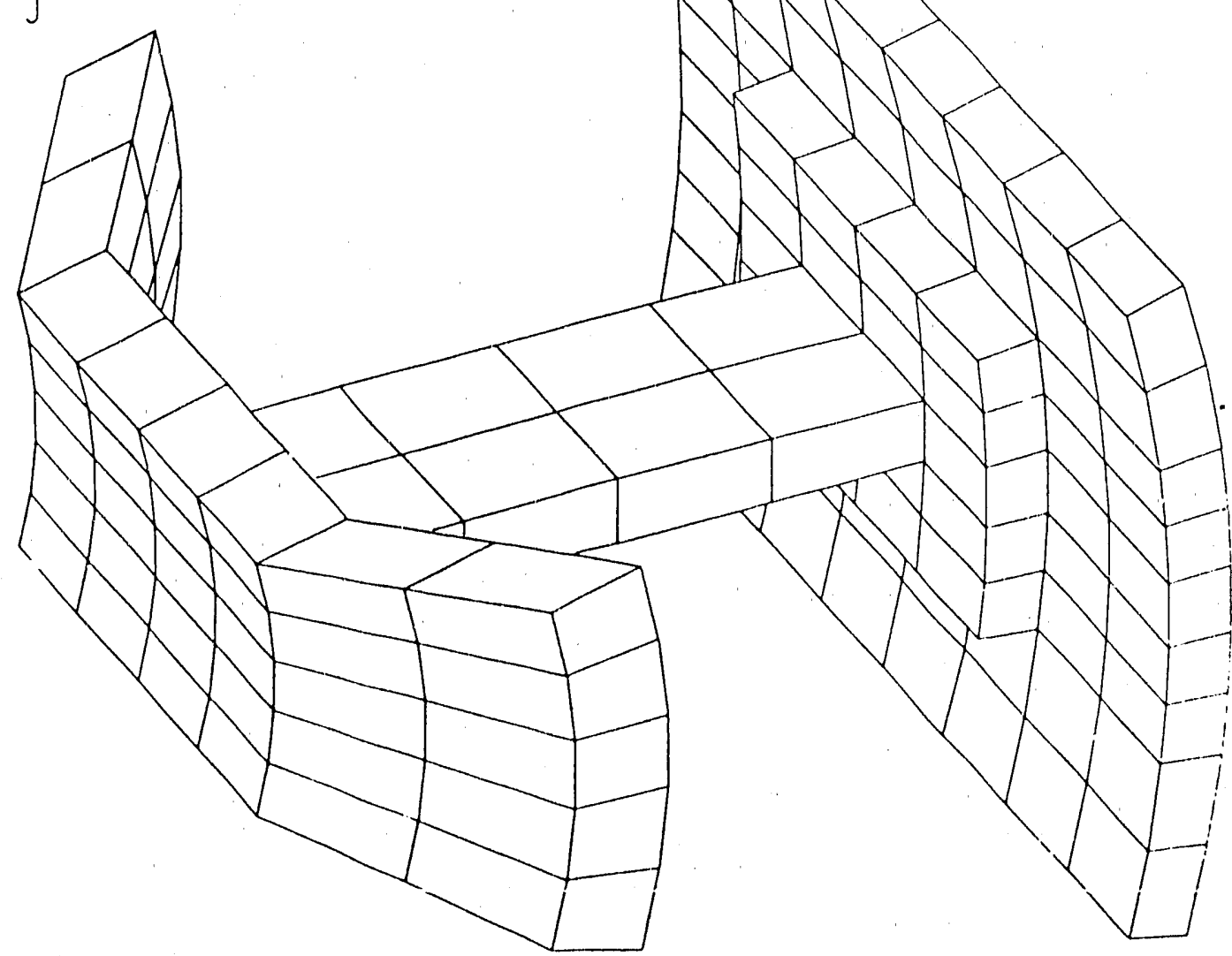


dn3d

sd 1 cone $\theta \quad 0 \quad 0 \quad 0 \quad 011035.311$

sd 2 cone $\emptyset \cdot 0 \quad 0 \quad 0 \quad 0 \quad 1.5 .667-35.311$

sd 3 cone $0 \quad 0 \quad 00001.75 \quad 35.311$

sd 4 cone $000.00016 .417-35.311$

sd 5 plan 0

sd 6 plan 0

gct 1:csca .386666666 mz 2:

lev 1 grep 0 1: ;

lev 2 levct 1:rxy mz -20: grep 0: ;

ps I 2

ps IV 1

start

$126778: 135568$ 10:1 344679 :

$\begin{array}{lllllllllll}4.25 & 5 & 10 & 10.5 & 11 & 0 & 8 & 13.5 & 16.5 & 22 & 30\end{array}$

0253566

cyli

di 2 3:1 3 o 4 4 6: ;

di 2 3: $: 1304$ : 3 :

di 3 4:1 2005 6: :

di $34: 25: 12$ 0 5 6:

di $12: 12007$ 6: :

$p b \begin{array}{lllllllll}1 & 2 & 0 & 2 & 2 & 0 & 2 & 0\end{array}$

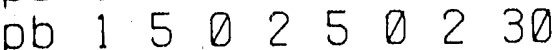

$m b 2302302-1.3+j 121.3$

sf 101102 sd $2+k 4$ sd 1

sf 201202 sd $4+k 4$ sd 3

sf 0

sf $\theta \cdot 0 \quad 6 \quad 0 \quad 0 \quad 6$ sd 6

$c P 1 \quad 120150 j$

Ict 11:rz 30: repe 11:

Irep 01123445678891011 :

end

pp IV

$p p \mid v$

start

1. 2:161:160:

$1.41 .64333333330360-244$

cyl,

end

end

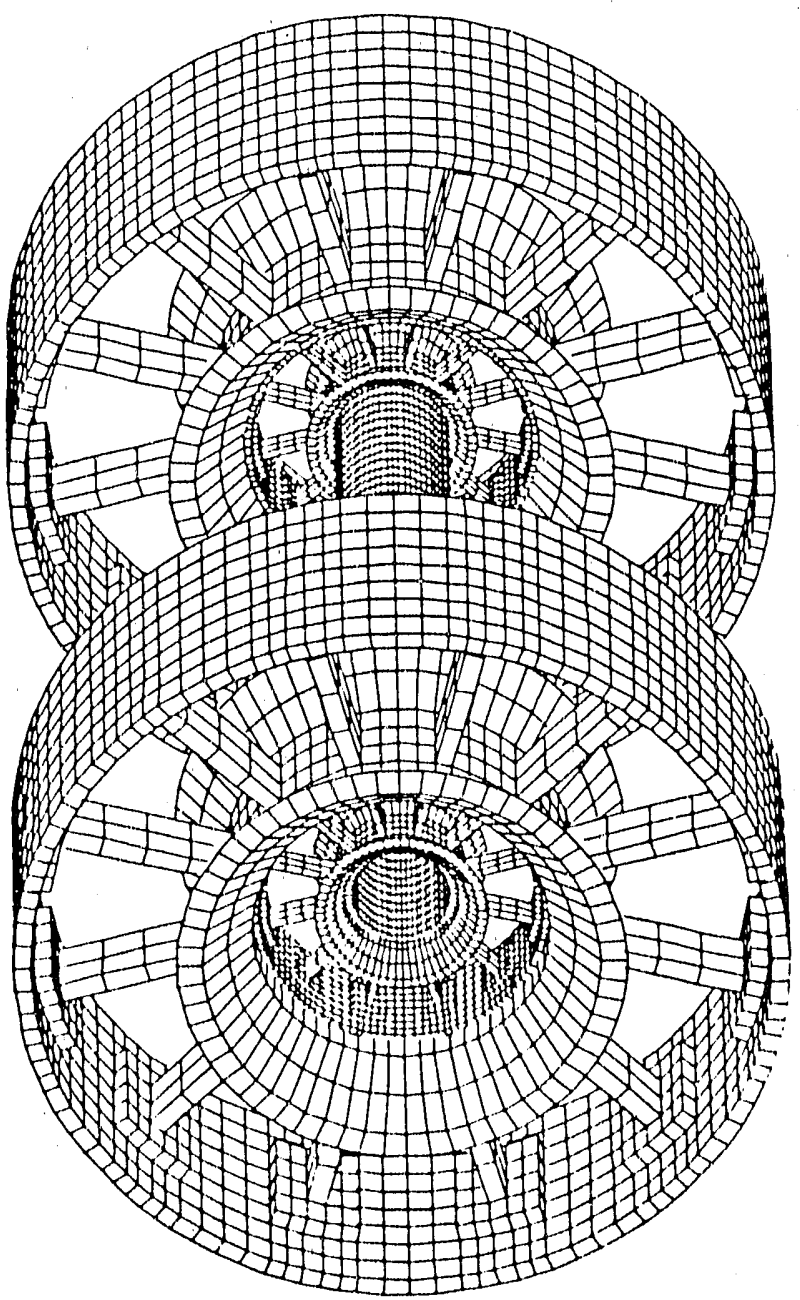


ron carr's sample problem

\section{$n k 3 d$}

sd 1 cy 0

sd 2 cy $\quad 0 \quad 0 \quad 0 \quad 0 \quad 0 \quad 120$

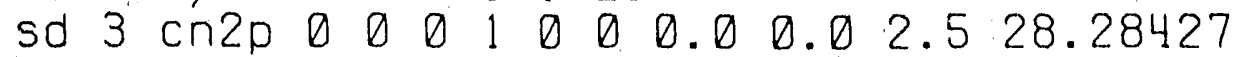

so 4 cy 0

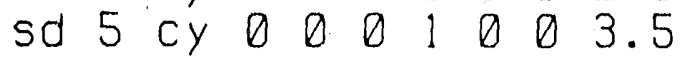

sd 6 plan 28.427 0.0 0.0100

sd 7 plan $000-0.670867+0.7415780$

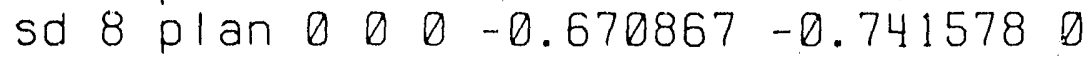

plan 3

008000010.001 symm

$00-80.010 .001$ symm

28.427001000 .001 symm

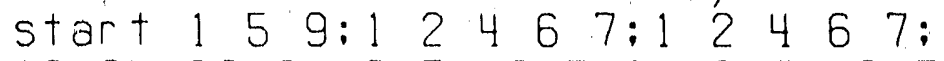

$102428.3-3.5-3.50+3.5+3.5$

$-3.5-3.50+3.5+3.5$

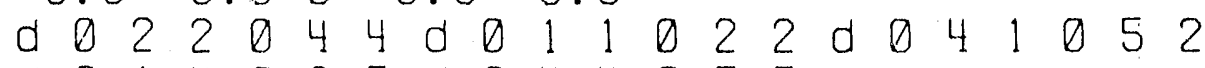
d 01140025 d 0444005 sf 1 l 0

sf 200200 sd 2 sf 300300 sd 6 sf 0100010 sd 5 sf 05000050 sf 00101001 sd 5 sf 00050 sf 0222024 sd 3 sf 04220444 sd 3

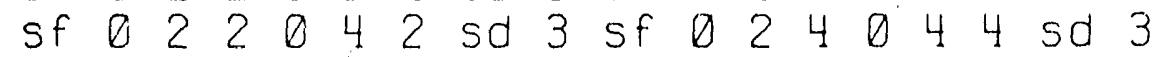
pr $1 \quad 0 \quad 0 \quad 1 \quad 0 \quad 0 \quad 1-200 \quad 0 \quad 000$ end

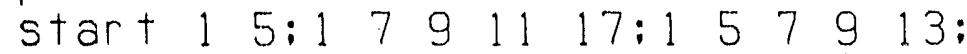

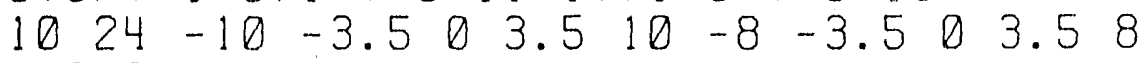
d 0222044

sf 0222024 sd 5 sf 0422044 sd 5 sf 0222042 sd 5 sf 02240444 sd 5 sf 1000100 sd 1 sf 2000200 sf 0100010 sd 8 sf 0500050 sd 7

pr $1 \quad 0 \quad 0 \quad 1 \quad 0 \quad 0 \quad 1 .-2000000$ end

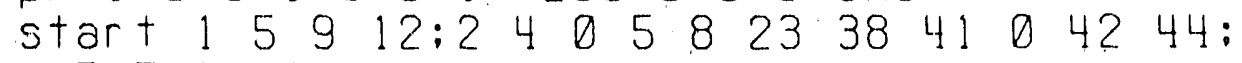

1579 13:

$\begin{array}{llllllllllll}10 & 20 & 24 & 25 & 42.1340 & 45 & 0 & 45 & 47.86598 & 180\end{array}$

$312.1340 \quad 31500315 \quad 317.86598 \quad-8 \quad-3.5003 .5 \quad 8$

d 25004770

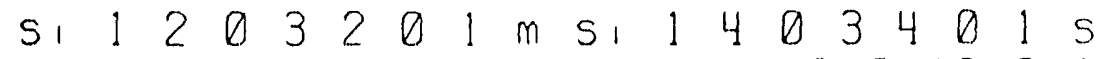

si 180038001 s si 1100031001010

pr $12 \begin{array}{llllllllllll} & 2 & 0 & 3 & 2 & 0 & 1 & 200 & 10 & 0 & 0\end{array}$

pr $14 \begin{array}{llllllllllllll} & 4 & 0 & 3 & 4 & 0 & 1 & -200 & 10 & 0 & 0\end{array}$

pr $18 \begin{array}{lllllllllllllllll} & 8 & 0 & 3 & 8 & 0 & 1 & -200 & 10 & 0 & 0\end{array}$

pr 111000310012001000

$\begin{array}{lllllllllllll}\text { pr } & 1 & 0 & 0 & 1 & 0 & 0 & 1 & -200 & 0 & 0 & 0\end{array}$

t $160160111111 \mathrm{cy} / 1$ end end 
wumore machine model

dn $3 d$

para ri $0.45 \quad 021.0 \quad r .31 .25 \quad 441.875:$

$\begin{array}{lllllllllllllllllllllll}\text { para } & z 1 & -2 & z 2 & 0 & z 3 & 1.5 & z 4 & 6.25 & z 5 & 7.75 & z 6 & 10.0 & z 7 & 11.0 & z 8 & 25.7\end{array}$

vara $\times 10 \times 2-1.326 \times 3-2.0625 \times 4-4.48125 \times 5-6.9 \times 6-17.26$;

vara y1 0 y 21.326 y.32.6875 y4 6.875 ;

start 2. 3:2 10:2 $356:$

$\%$ \% $3 \%$ + $0360 \% 22 \% 23 \% 24 \% 25$

mate i cyl, end

start? $24: 23-4:-2-3:$

$\% \times 1 \% \times 2 \div \times 3 \div y 1 \% y 2 \% y 3 \% \geq 3 \% \geq 4$

ac $2112=23000 \% 4001$

ac $1212223000 \% 4001$

d $11: 222$

mate 2 thick 0.25 end

start $24 \quad-5-6:-2 \quad 3 \quad 4 \quad-5:-2 \quad-3$;

$\% \times 6 \% \times 5 \% \times 4 \% \times 3 \%$ \% $\% 2 \%$ \% $\%$ y 0.6257 .125

d $21: 312$

b $31: 412111111$

$\begin{array}{lllllllll} & 1 & 0 & 0 & 0 & 2.7 & 3.7 & 4.7 & 5.75\end{array}$

$\begin{array}{lllllll}k & 3 & 0 & 3 & 0 & 1.0625 & 6.6875\end{array}$

$k 4030 \% 23 \% 24$

mate 2 thick 0.25 end

start : $-2: 2 \quad 10:-12356-7$ :

$\%$ r $1 \%$ r $0360 \% z 1 \% z 2 \% z 3 \% z 4 \% z 5 \% z 6$

mate 3 thick 0.5 cyl, end

start $\vdots-2: 2 \quad 10: 12-6$ :

$\% r 1 \% r 20360 \% z 6 \% z 7 \% z 8$

$p+2 \& 11 \%$ r 3

mate L: thıck 0.3 cyl, end

start 2 3:2 3:1 7 15:

$-11-i \quad 1 \% z 1 \% z 6 \% z 8$

a 11 ! $2233 \% 1$

sate 1 end end

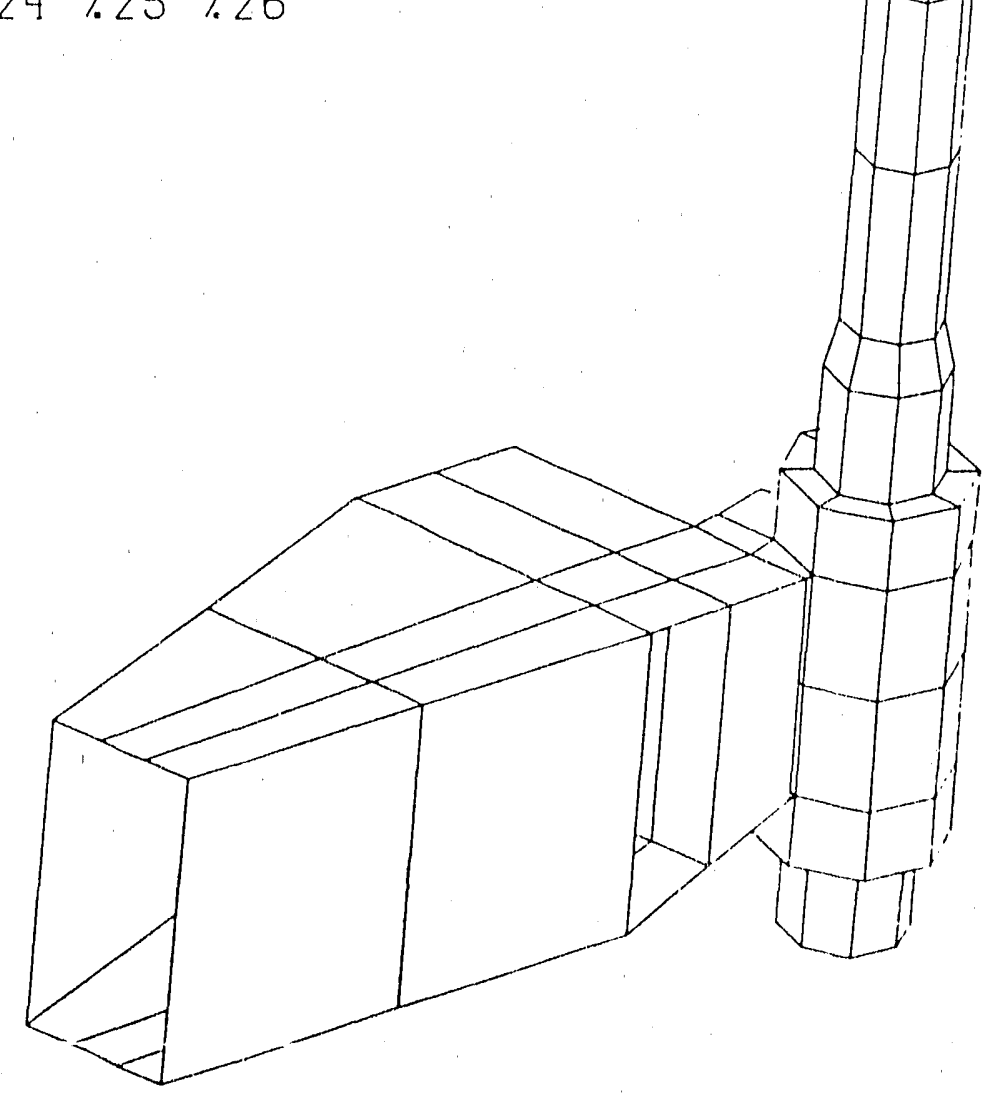


a simple sprocket

coor 1

$r+2000 r+2100 r+2010$

start

$12345: 13$ 5:1 3:

$\begin{array}{lllllllllll}-1.2 & -0.6 & 0 & 0 & .6 & 1.2 & 10 & 11 & 12 & 0 & .5\end{array}$

d 220430

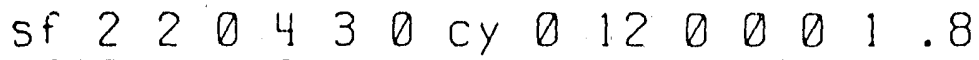

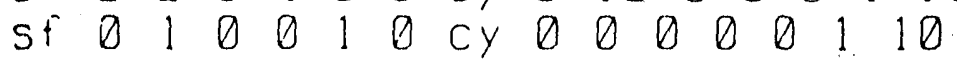

sf $0 \begin{array}{llllllllllllll} & 0 & 0 & 0 & 3 & 0 & c y & 0 & 0 & 0 & 0 & 0 & 1 & 12\end{array}$

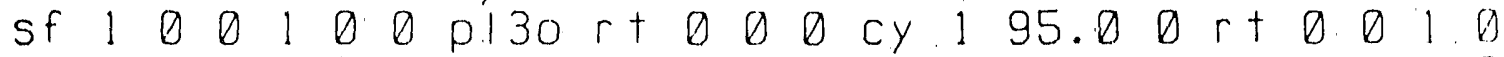

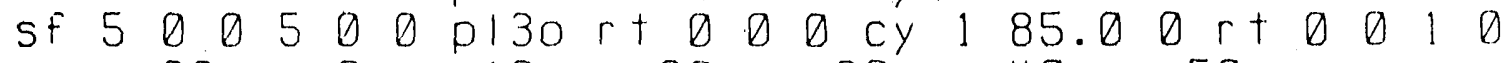

cocr 36:rz D:rz 10:rz 20:rz 30:rz 40:rz 50

:rz 60:rz 70:rz 80:rz 90:rz 100:rz 110

:r= 120:rz 130:rz 140:rz 150:rz 160:rz 170

:rz 180:rz 190:rz 200:rz 210:rz 220:rz 230

:rz 240:rz 250:rz 260:rz 270:rz 280:rz 290

: rz 300:rz 310:rz 320:rz 330:rz 340:rz 350:

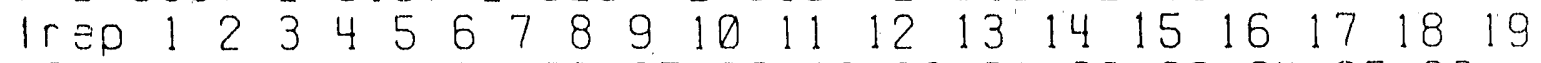

$\begin{array}{llllllllllllllllll}2 E & 21 & 22 & 23 & 24 & 25 & 26 & 27 & 28 & 29 & 30 & 31 & 32 & 33 & 34 & 35 & 36:\end{array}$

eñ

start

$12345: 135: 13$;

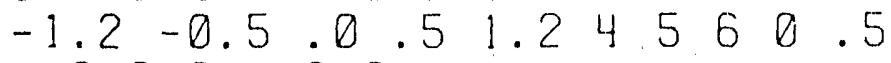

d 220430

sf $22 \begin{array}{lllllllllllllll} & 2 & 0 & 3 & 0 & c y & 0 & 6.0 & 0 & 0 & 0 & 1 & .8\end{array}$

Sf $0 \begin{array}{llllllllllllllllllll} & 0 & 0 & 0 & 1 & 0 & C Y & 0 & 0 & 0 & 0 & 0 & 1 & 4 . & 0\end{array}$

sf $\theta \begin{array}{llllllllllllll} & 0 & 0 & 3 & 0 & c y & 0 & 0 & 0 & 0 & 0 & 1 & 6.0\end{array}$

sf $1001000130 r+000$ cy $1100.00 r+0010$

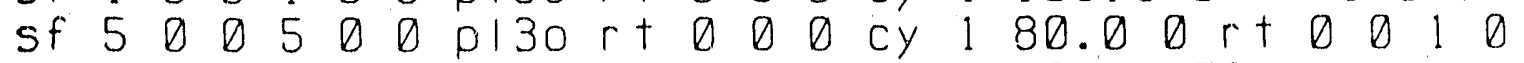

coor 36:rz $0: r z$ 10:rz 20:rz 30:rz 40:rz:50

irz 60:rz 70:rz 80:rz 90:rz 100:rz 110

:rz 120:rz 130:rz 140:rz 150:rz 160:rz 170

:rz 180:rz 190:rz 200:rz 210:rz 220:rz 230

:rz 240:rz 250:rz 260:rz 270:rz 280:rz 290

: rz 300:rz 310:rz 320:rz 330:rz 340:rz 350:

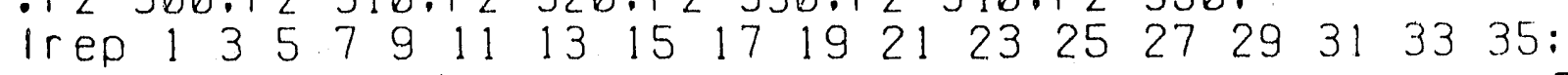

Irepe 1 : end end 
- hreaded bolt

dn $3 d$

start 1 4:1 $59913 \quad 1721 \quad 25: 1 \quad 3$ :

.25 .4500000000000 .2

$\begin{array}{llllllllll}r & 0 & 2 & 0 & 0 & 2 & 0 & r z & 60:\end{array}$

$\begin{array}{rlllllllll}r & 0 & 3 & 0 & 0 & 3 & 0 & r z & 120:\end{array}$

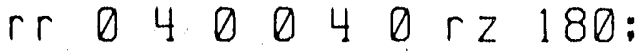

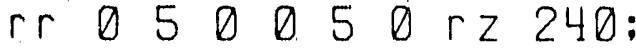

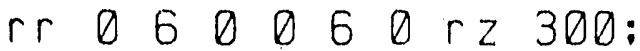

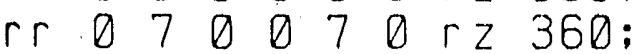

sf 1000100 Cy 0

end

start 1 $7: 1 \quad 7: 13 \quad 10$;

$\begin{array}{llllllll}-1 & 1 & -1 & 1 & 0 & .2 & 1.5\end{array}$

sf $1-1-1-2:-1-2: ; c y \quad 0 \quad 0 \quad 0 \quad 0001.25$

end

start 12 3:1 501:12 2 ;

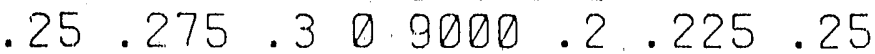

d 2002303

pa 2 b 1 z $\quad .2125$

pa $30011 z$

pa $202 \times .27$

pa $200 \quad 3 \times z \quad .275 \quad .2375$

pa $300.2 \times z \quad .275 .2375$

ma $0.20 \geq 1.25$

cy 1

end end

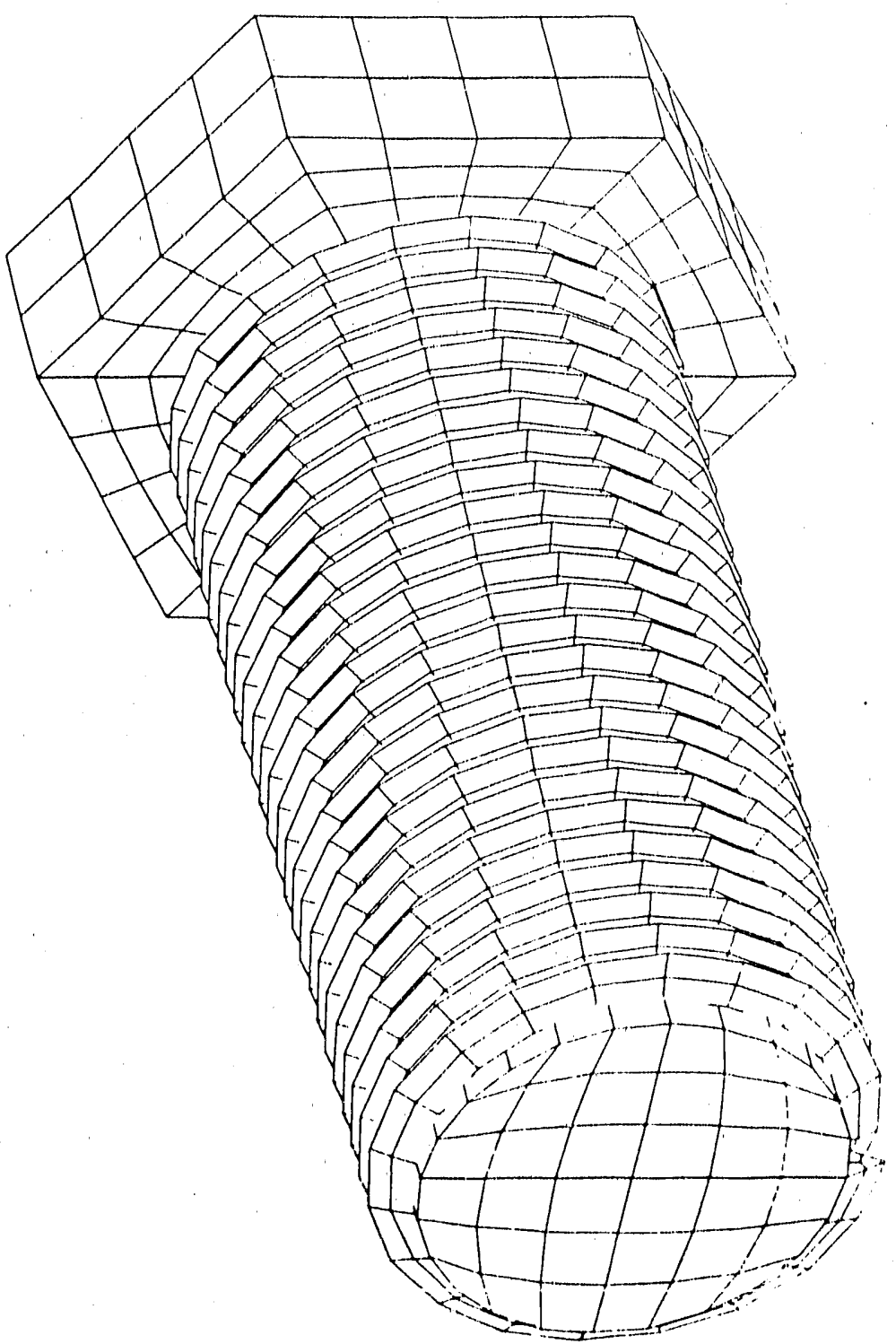




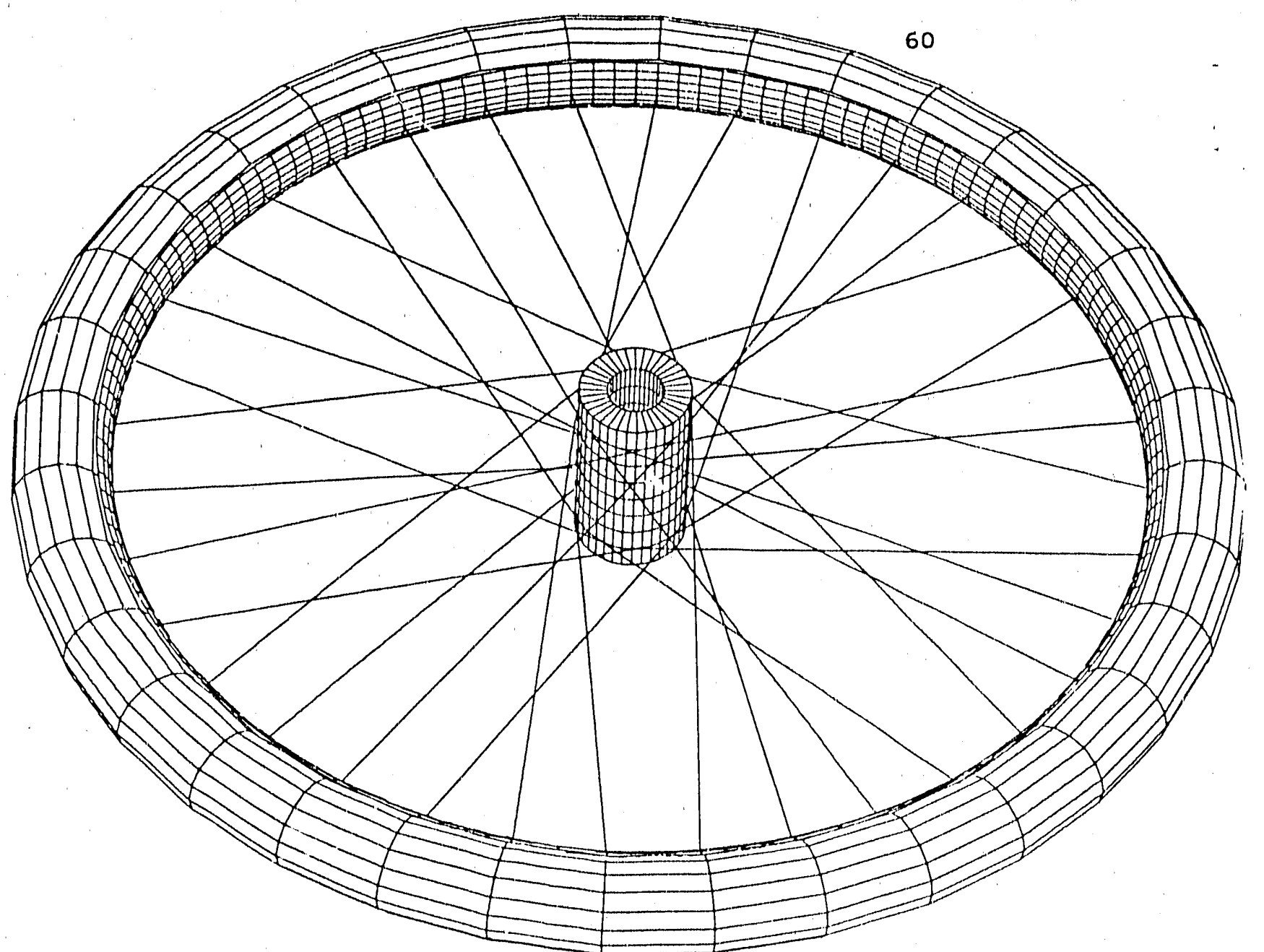

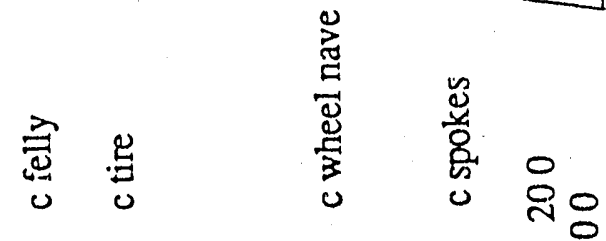

旨

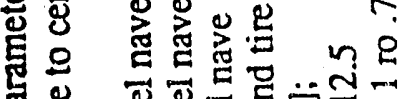

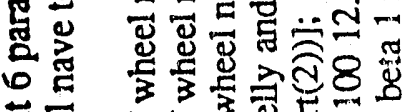

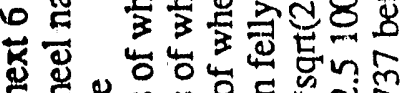

.

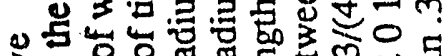

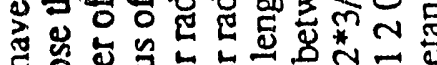

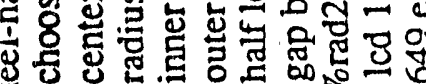

政

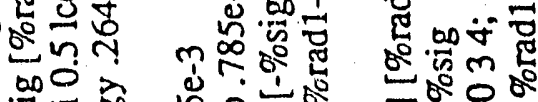

5를

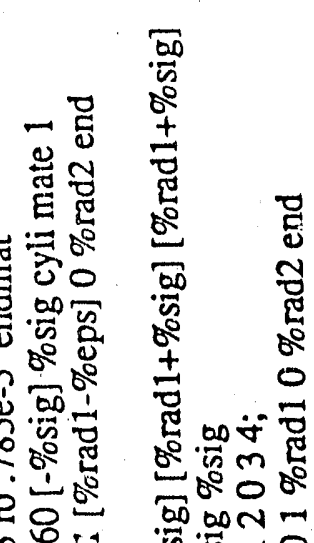

(2)

苋哭

觉 $80^{\circ}$

듕ㅇㅇㅇ

岛 30

पू

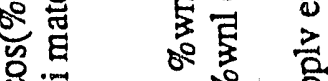

装

古察

每

可高等

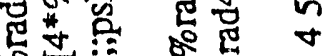

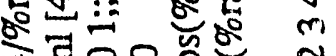

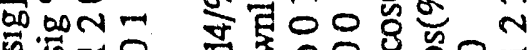

"⿺辶一巛 oㄴ.

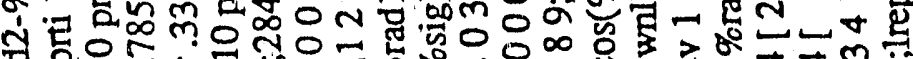

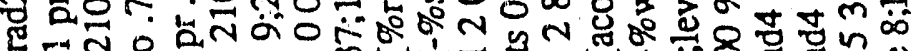

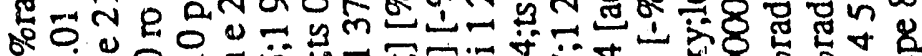

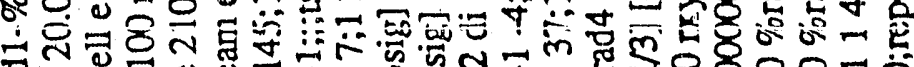

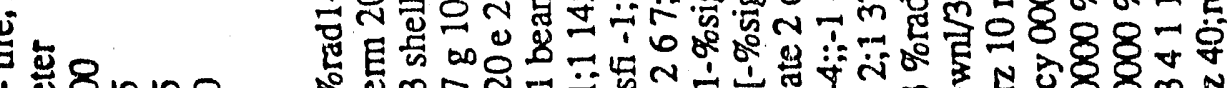

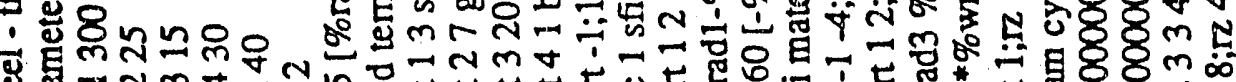

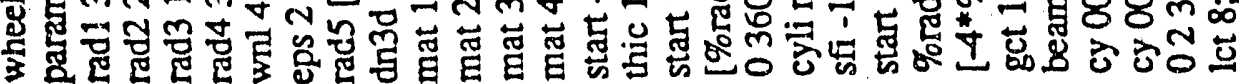


wood screw by Robert Ralnsberger

sd 1 sp 0002 sd 2 plan 001001

start 15 1321 25;1 511131521 25;1 5 9;

$-.7-.70 .7 .7-.5-.5-.30 .3 .5 .50 .5 .5$

di $12045 ; 17 ; 2$ 3;di 1 5;1 $2067 ; 23$;

d 232453 di $12045 ; 12067 ; 1$ 2;

sfi $-1-5 ; 2$ 6;1 2;sd 1 sfi 2 4;-1 -7;1 2;sd 1

sfi $24 ; 23056 ;-3 ;$ sd 1

sf $002002 \mathrm{sd} 2 \mathrm{sf} 001001$ plan 000001

sfi $24 ;-2-6 ; 2$ 3;sd 2 sfi $-2-4 ; 23056 ; 23 ;$ sd 2

sf 030030 plan $0-.30010$

sf 050050 plan 0.30010

edit

$z=-.75^{\star} z-.2$

end

start $12 ; 137 ; 13 ; 0.90360-.20$ cyli

edit

fir 1122372

$z=-.1+.2^{*}(j-1) / 36$

fir 1132373

$z=.4^{*}(j-1) / 36$

end

start $123 ; 11081 ; 123 ; 0.91 .30108000 .2 .4$

d 202303 cyli

edit

$z=z+(j-1)^{*} .4 / 36$

fir 311310811

$z=z+.09$

fir 312310812

$z=z-.09$

cyli

fir 111310813

phr $0-99999669999950$

$x=x^{\star}\left(1-.15^{*}(z-6)\right)$

phr 0 -99999 969999950

$z=(z-9)^{*}(1-(z-9) / 8)+9$

end

end
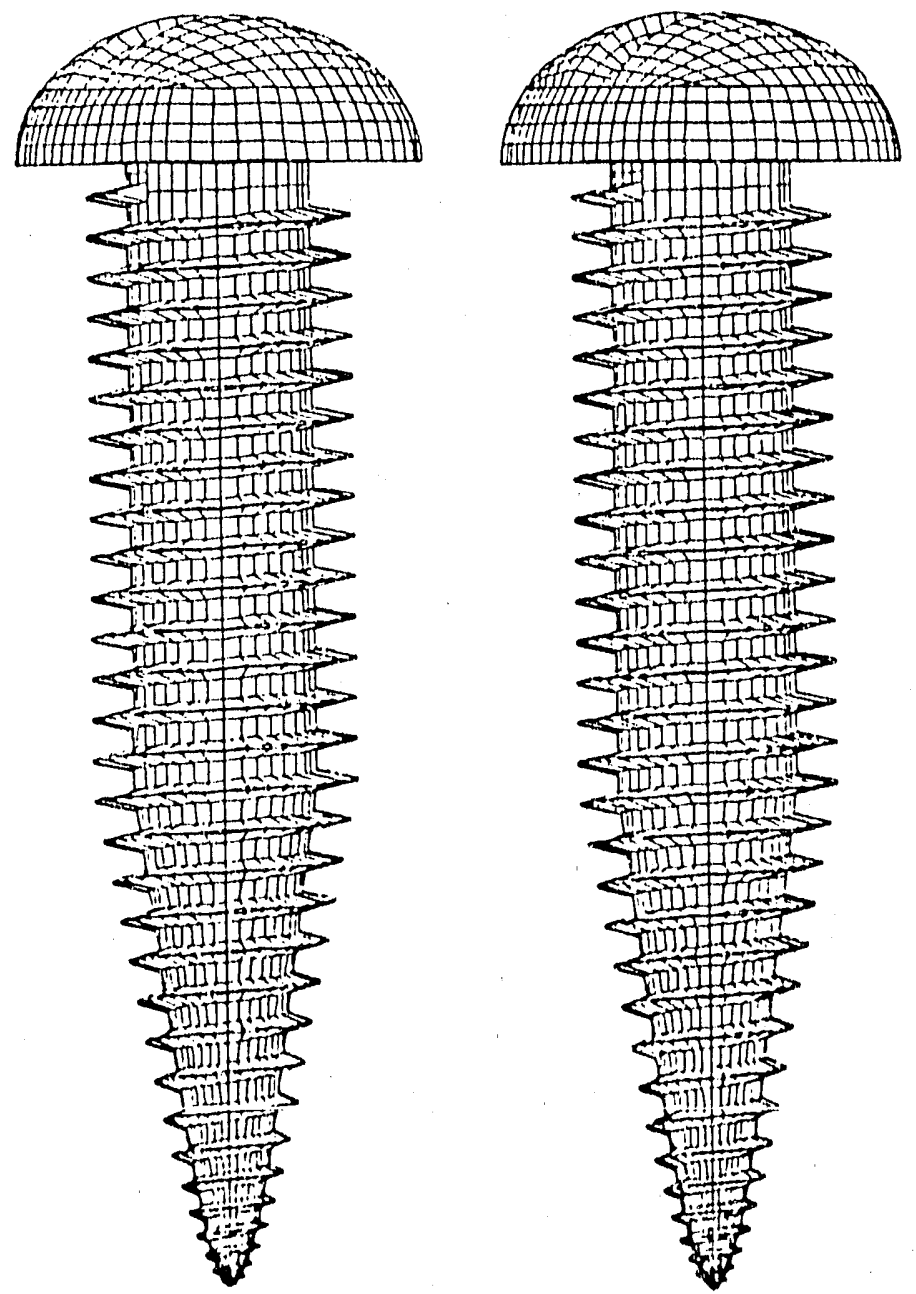
connecting rod

sd 1 cy 0

sd 2 cy 00050100.0

sd 3 cy -50190108.0

sd $4 c y+2001601014.0$

sd 5 cy -8009001019.0

start

$12 ; 6: 123567: 12568$;

$\begin{array}{lllllllllllllll}-5 & -5 & 5 & 5 & -12 & -8 & -4 & 4 & 8 & 12 & 10 & 10 & 0 & 0 & -5\end{array}$

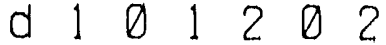

d 30011400

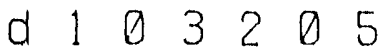

d $300 \begin{array}{lllll} & 3 & 4 & 0 & 5\end{array}$

$\begin{array}{llllllllll}2 & 2 & 0 & 2 & 3 & 0 & 3 & 2 & 5.0\end{array}$

$\begin{array}{llllllllll}a & 1 & 0 & 1 & 4 & 0 & 4 & 2 & 8.0\end{array}$

d 2002303 end

start

$1256: 123567: 12568$ :

$\begin{array}{lllllllllllllll}-10 & -10 & 10 & 10 & -12 & -8 & -4 & 4 & 8 & 12 & 105 & 105 & 125 & 125 & 135\end{array}$

d 100101200

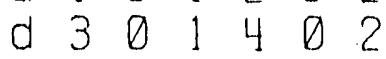

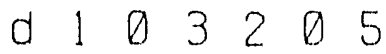

d $300 \begin{array}{llllll} & 3 & 0 & 4 & 0 & 5\end{array}$

$\begin{array}{lllllllllllll}2 & 2 & 0 & 2 & 3 & 0 & 3 & 2 & 15.0\end{array}$

$\begin{array}{lllllllllll}0 & 1 & 0 & 1 & 4 & 0 & 4 & 2 & 17.0\end{array}$

d $2002 \quad 3003$ end

start

$13578 \quad 1012: 12455: 16991720$ :

$\begin{array}{lllllllllllllllllll}0 & 10 & 15 & 17 & 19 & 23 & 25 & -8 & -4 & 4 & 8 & 122 & 100 & 90 & 18 & 13\end{array}$

d 100101402

d $300 \begin{array}{lllll} & 3 & 7 & 0 & 5\end{array}$

d $5 \begin{array}{llllll}5 & 2 & 6 & 3 & 3\end{array}$

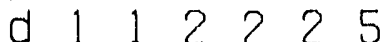

d $1 \begin{array}{llllll} & 3 & 2 & 2 & 4 & 5\end{array}$

$\begin{array}{llllllllll}2 & 5 & 2 & 1 & 6 & 3 & 3 & 3 & 3.0\end{array}$

sf 4001402 sd 1

sf $100 \begin{array}{llllll}2 & 4 & 0 & 2 & \text { sd } & 1\end{array}$

sf $2004 \begin{array}{llllll} & 2 & 0 & 5 & \text { so } & 3\end{array}$

sf $3004 \quad 3 \quad 0 \quad 5$ sd 4

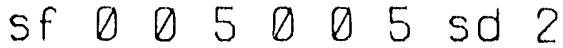

sf 202203 sd 5

sf $0 \begin{array}{lllllllllllll} & 0 & 1 & 0 & 0 & 1 & \text { plan } & 0 & 0 & 122 & 0 & 0 & 1\end{array}$

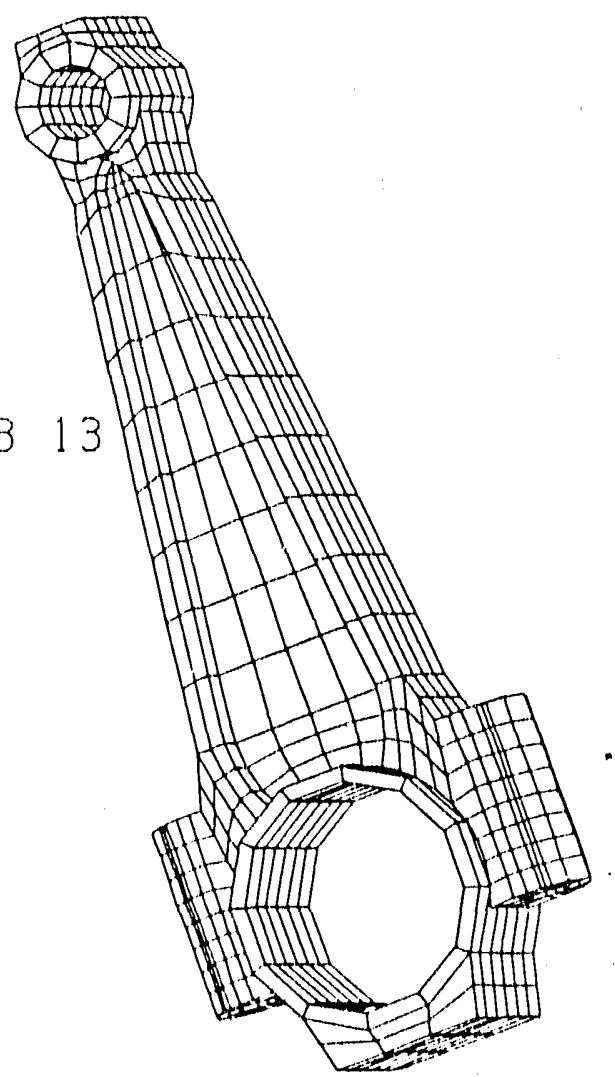

$m b \quad 4 \quad 0 \quad 2.700307 .0$

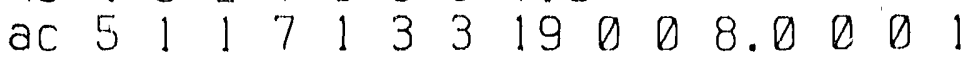

ac $5 \begin{array}{lllllllllllllll} & 5 & 4 & 1 & 7 & 4 & 3 & 3 & 19 & 0 & 0 & 8.0 & 0 & 0 & 1\end{array}$

ac $70 \begin{array}{llllllllllllll} & 7 & 0 & 1.7 & 0 & 3 & 3 & 19 & 0 & 0 & 8.0 & 0 & 0 & 1\end{array}$

coor $1 r+\theta \theta \theta r+-10 \theta r+\theta-10$ lrep $\theta 1$ i end end 

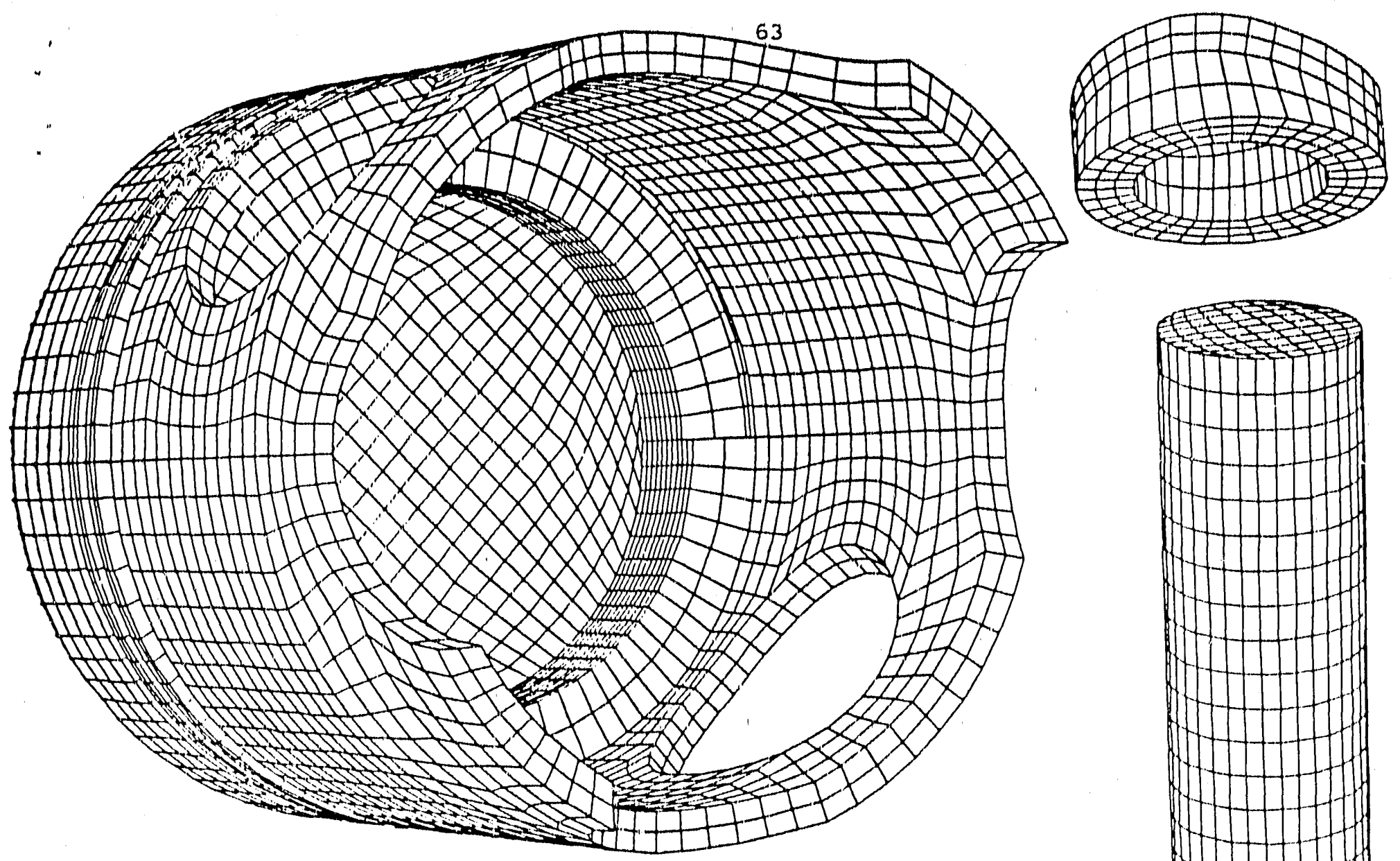

要学

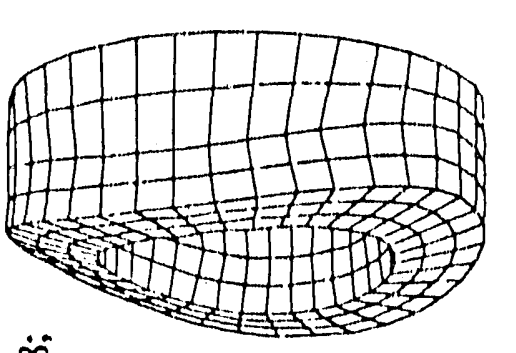

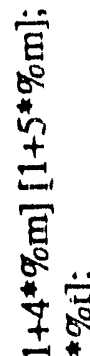

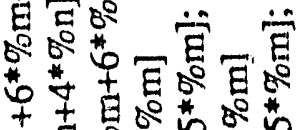

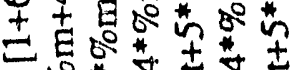

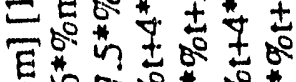

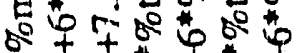

요

$0= \pm m=m$ n

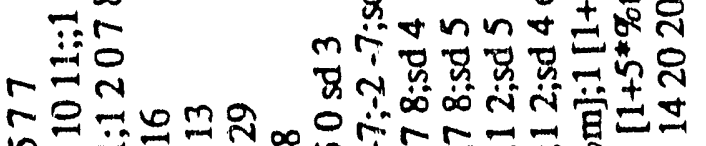

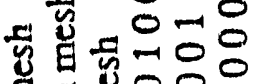

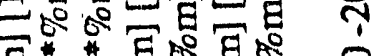

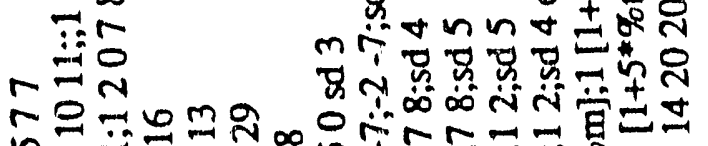

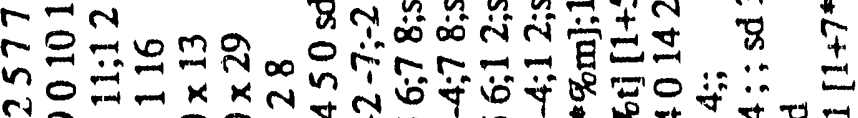

no

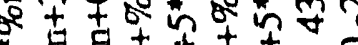

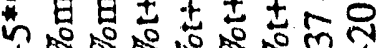

붕ㅇㅇ응

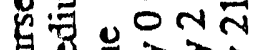

格

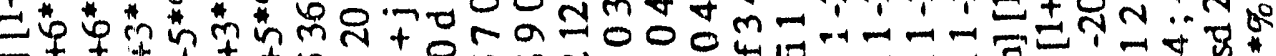

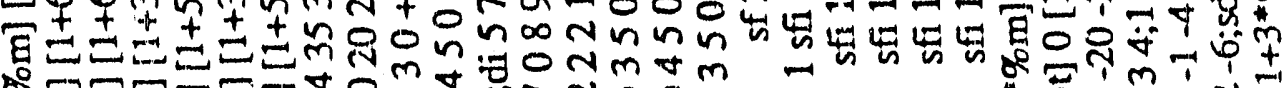

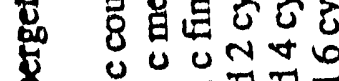

-

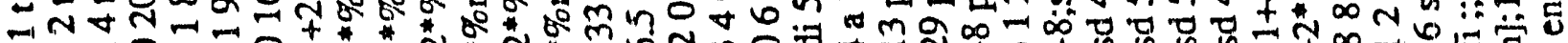

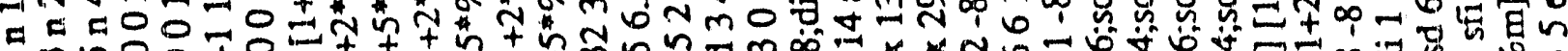

NmnO0 10

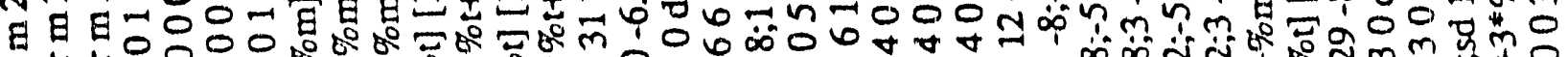

t t to 0000 Der

ลे \&

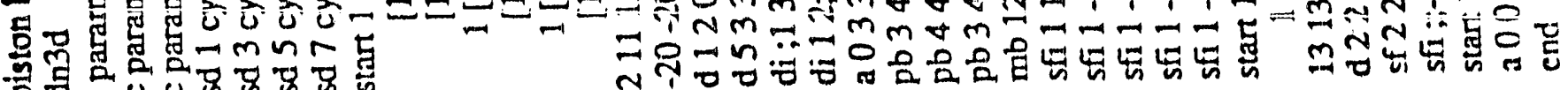

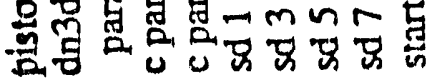




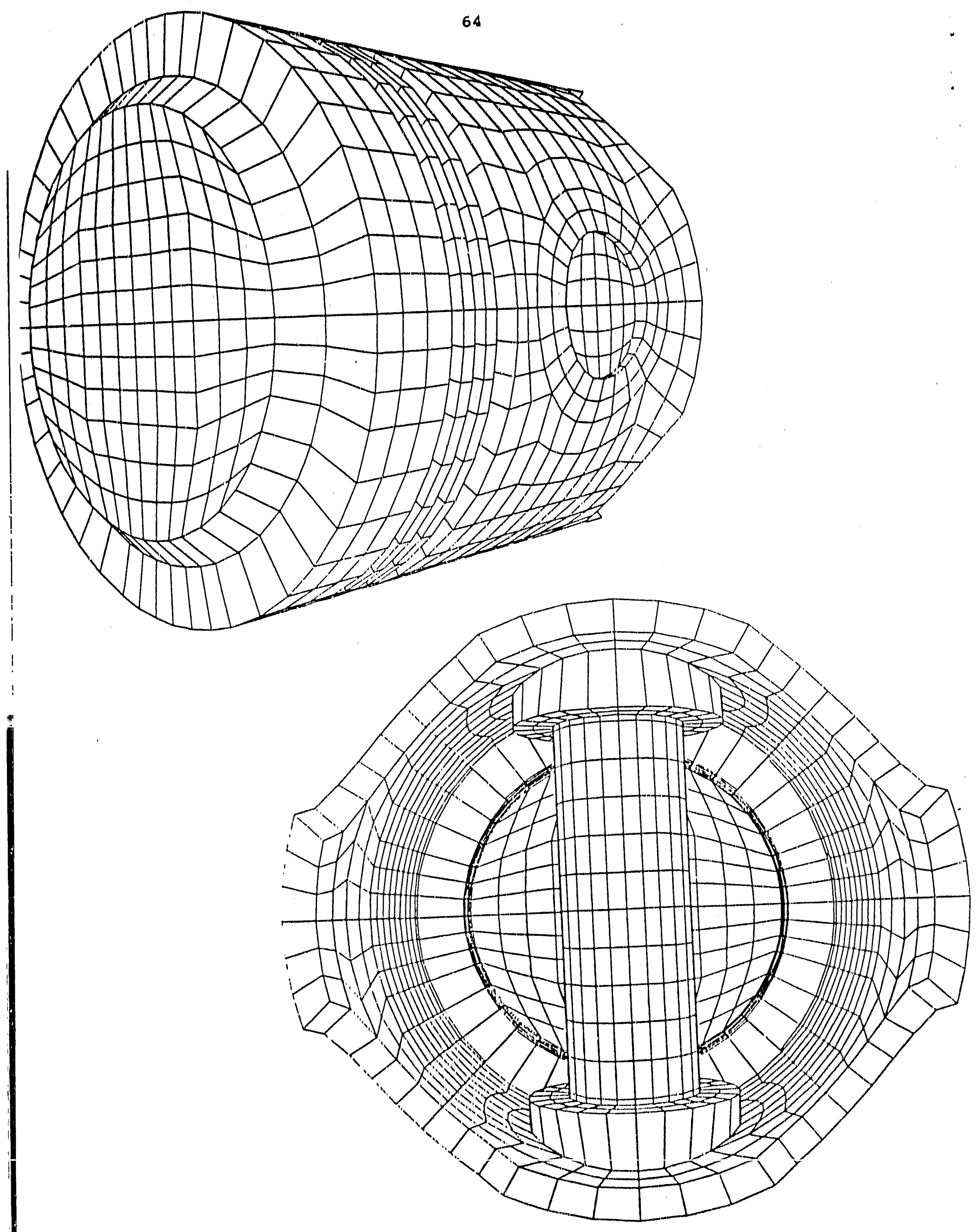



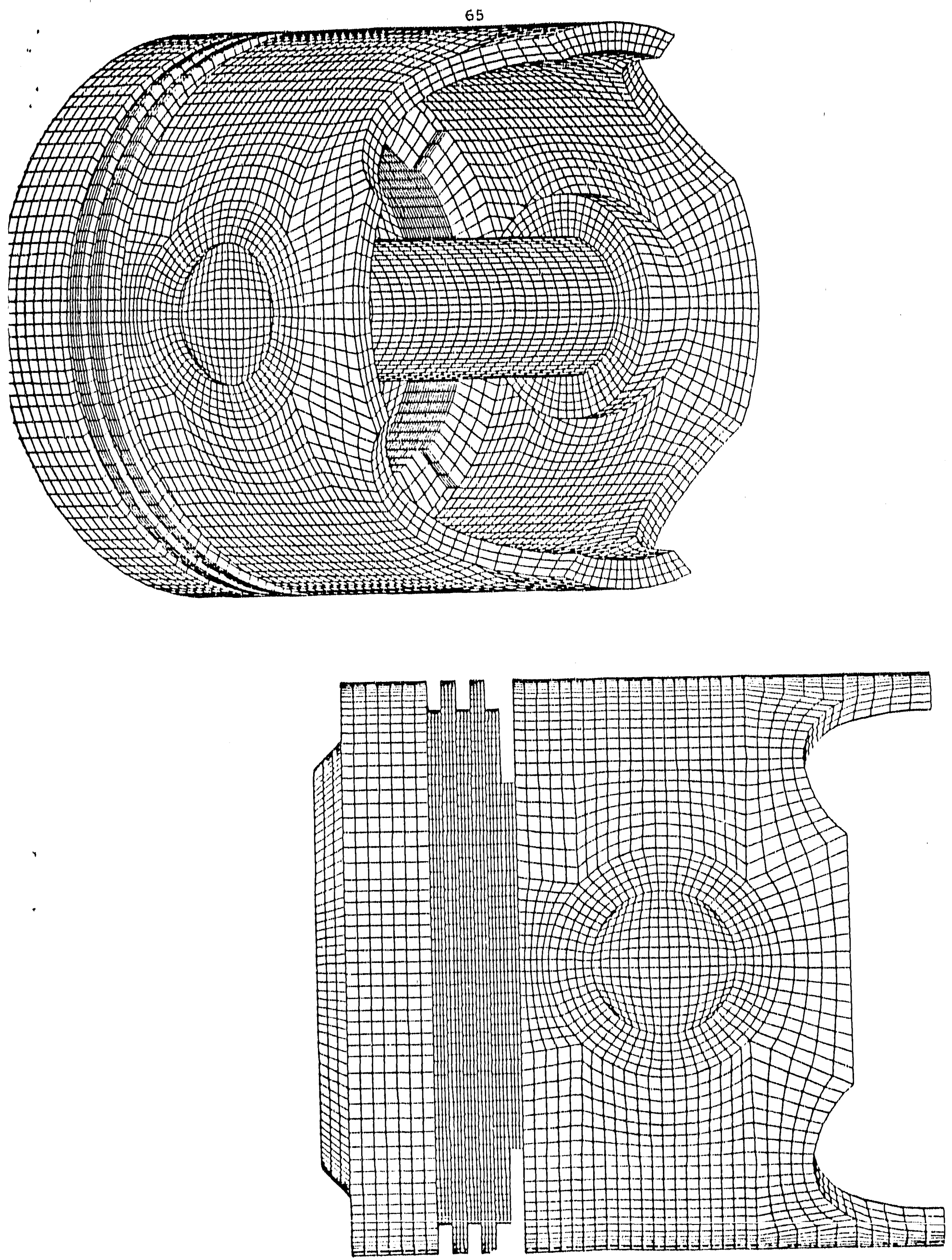
chain link

coor $10 \mathrm{mx} 0: \mathrm{mx} 2.4: \mathrm{mx} 4.8: \mathrm{mx} 7.2: \mathrm{mx} 9.6:$

$m \times 12.0: m \times 14.4: m \times 16.8: m \times \quad 19.2: m \times 21.6:$

start $12467: 1256: 1256:$

$-1.2-1.201 .21 .2$

$-1.2-1.21 .21 .2-.75-.5 .5 .75$

$\begin{array}{lllllllllllllllllllll}1 & 0 & 2 & 2 & 0 & 3 & 0 & 4 & 0 & 2 & 5 & 0 & 3 & 0 & 0 & 1 & 2 & 0 & 2 & 3\end{array}$

$\begin{array}{lllllllllllllllllllll}0 & 3 & 2 & 0 & 4 & 3 & d & 1 & 1 & 0 & 2 & 2 & 0 & d & 1 & 3 & 0 & 2 & 4 & 0\end{array}$

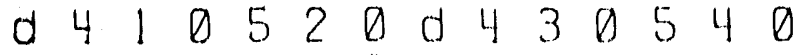

$\begin{array}{lllllllllll}0 & 2 & 2 . & 0 & 4 & 3 & 0 & 3 & 0.8\end{array}$

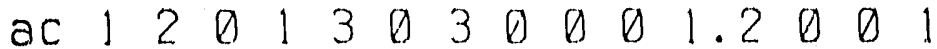

$\begin{array}{llllllllllllllllllll}a c & 2 & 1 & 0 & 3 & 1 & 0 & 3 & 0 & 0 & 0 & 1.2 & 0 & 0 & 1\end{array}$

$\begin{array}{llllllllllllllll}a c & 2 & 4 & 0 & 3 & 4 & 0 & 3 & 0 & 0 & 0 & 1.2 & 0 & 0 & 1\end{array}$

repe 13579 ; end

start 1 2467:1256:1256:

$1.21 .200-1.2 \quad-1.2$

$-1.2-1.21 .21 .2-.75-.5 \quad .5 \quad .75$

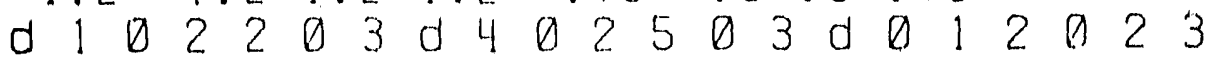

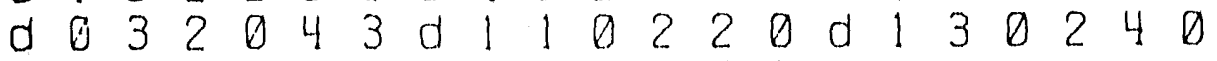

d 4100520 d 43305540

$\begin{array}{llllllllll}a & 2 & 2 & 0 & 4 & 3 & 0 & 3 & 0.8\end{array}$

ac $12 \begin{array}{llllllllllllll}2 & 0 & 1 & 3 & 0 & 3 & 0 & 0 & 0 & 1.2 & 0 & 0 & 1\end{array}$

$\begin{array}{llllllllllllllll}a c & 2 & 1 & 0 & 3 & 1 & 0 & 3 & 0 & 0 & 0 & 1.2 & 0 & 0 & 1\end{array}$

$\begin{array}{llllllllllllllll}a c & 2 & 4 & 0 & 3 & 4 & 0 & 3 & 0 & 0 & 0 & 1.2 & 0 & 0 & 1\end{array}$

coor $1 r+2.400 r+2.500 r+2.410$

Irep 1:repe 13579 : end

start $12467: 1256: 1256$ :

$-1.2-1.2$ D 1.21 .2

$-1.2-1.21 .21 .2-.5-.25 .25 .5$

$\begin{array}{lllllllllllllllllllll}1 & 0 & 2 & 2 & 0 & 3 & d & 4 & 0 & 2 & 5 & 0 & 3 & d & 0 & 1 & 2 & 0 & 2 & 3\end{array}$

$\begin{array}{lllllllllllllllllllll}d & 0 & 3 & 2 & 0 & 4 & 3 & d & 1 & 1 & 0 & 2 & 2 & 0 & d & 1 & 3 & 0 & 2 & 4 & 0\end{array}$

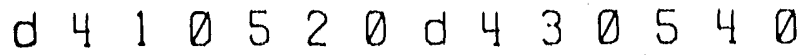

$\begin{array}{llllllllllllllll}2 & 2 & 2 & 0 & 4 & 3 & 0 & 3 & .8 & 8 & 2 & 2 & 0 & 4 & 3 & 0\end{array}$

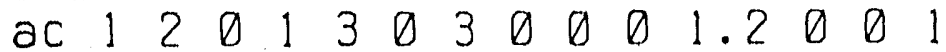

$\begin{array}{llllllllllllllll}a c & 2 & 1 & 0 & 3 & 1 & 0 & 3 & 0 & 0 & 0 & 1.2 & 0 & 0 & 1\end{array}$

$\begin{array}{llllllllllllllll}a c & 2 & 4 & 0 & 3 & 4 & 0 & 3 & 0 & 0 & 0 & 1.2 & 0 & 0 & 1\end{array}$

repe 246810 iend

start $12467: 1256: 1256:$

$1.21 .20-1.2-1.2$

$-1.2-1.21 .21 .2-.5-.25-25-5$

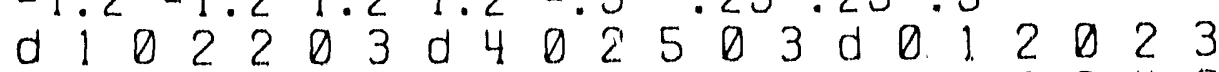

$\begin{array}{lllllllllllllllllllll}d & 0 & 3 & 2 & 0 & 4 & 3 & d & 1 & 1 & 0 & 2 & 2 & 0 & d & 1 & 3 & 0 & 2 & 4 & 0\end{array}$

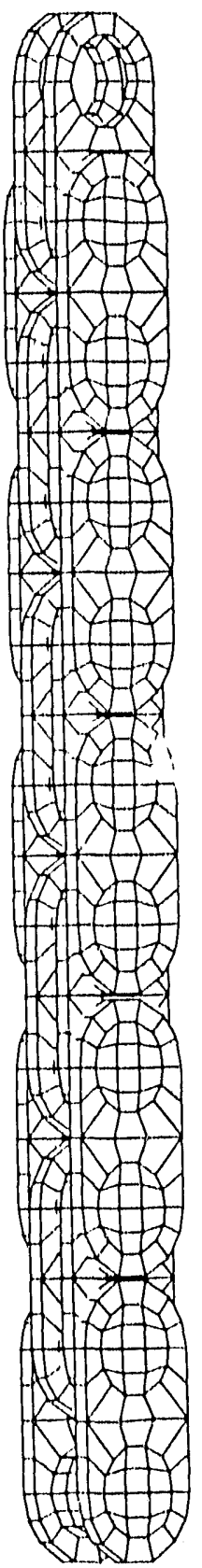

d 410520 d 45305540

$\begin{array}{llllllllllllllll}0 & 2 & 2 & 0 & 4 & 3 & 0 & 3 & .8 & \mathrm{~d} & 2 & 2 & 0 & 4 & 3 & 0\end{array}$ $\begin{array}{llllllllllllllll} & a c & 1 & 2 & 0 & 1 & 3 & 0 & 3 & 0 & 0 & 0 & 1.2 & 0 & 0 & 1\end{array}$ $\begin{array}{llllllllllllllll}a c & 2 & 1 & 0 & 3 & 1 & 0 & 3 & 0 & 0 & 0 & 1.2 & 0 & 0 & 1\end{array}$ $\begin{array}{llllllllllllllll}a c & 2 & 4 & 0 & 3 & 4 & 0 & 3 & 0 & 0 & 0 & 1.2 & 0 & 0 & 1\end{array}$

coor $1 r+2.400 r+2.500 r+2.410$

Irep 1:repe 2468 10: end end 
axisymmetric mirror problem

tole.01 exch 312

start $26 \begin{array}{lllllllll}6 & 8 & 9 & 10 & 11 & 12 & 13 & 14 & 14:\end{array}$

$2679910111314 \quad 16 \quad 17: 121:$

$-4.872-2.947-2.637-2.48-2.32-2.2$

$-2.0-1.8-.18309 .125 \quad 10.625 \quad 10.935$

$11.535 \quad 11.875 \quad 12.514 \quad 13.285 \quad 13.5$

14.5514 .750360

d $12 \begin{array}{lllllllllll} & 2 & 3 & 10 & 2 & d & 3 & 4 & 1 & 5 & 2\end{array}$

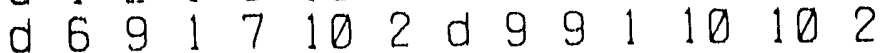

d 771882 d 8111082

j $40001-2.48-2.48-2.48-2.48$

$\begin{array}{llllll}0 & 0 & -2.137 & -2.137 & -1.975 & -1.975\end{array}$

j $30001-2.637-2.637-2.637-2.637$

0. $0-2.32-2.32-2.32-2.32$

$\begin{array}{lllllllllllllll}\text { ac } 2 & 2 & 0 & 3 & 2 & 0 & 3 & -2.947 & 10.935 & 0 & .31 & 0 & 0 & 1\end{array}$

$\begin{array}{llllllllllllllll}\mathrm{ac} & 3 & 2 & 0 & 3 & 3 & 0 & 3 & -2.947 & 10.935 & 0 & .31 & 0 & 0 & 1\end{array}$

J $8001-1.8-1.425-1.368-1.197$

$-1.112-.952-.755-.7-.7-.7$

J $7.001-1.983-1.608-1.55-1.38$

$-1.295-1.135-.938-.938-1.03-1.03$

J $6001-2.2-2.073-2.047-1.96$

$-1.916-1.664-1.565-1.565-1.285-1.285$

J $50001-2.32-2.32-2.32-2.32$

$-2.32-1.975-1.975-1.975-1.63-1.63$

$\begin{array}{llllllllllllllllll}\operatorname{ac} & 4 & 7 & 1 & 5 & 7 & 2 & 3 & -2.395 & 12.785 & 0 & .5 & 0 & 0 & 1\end{array}$

$\begin{array}{lllllllllllllllllll}\text { ac } & 5 & 6 & 1 & 5 & 7 & 2 & 3 & -2.395 & 12.785 & 0 & .5 & 0 & 0 & 1\end{array}$

pt $69 \begin{array}{lllll} & 9 & 12 & -1.285 & 14.650\end{array}$

pt $79012-1.03014 .650$ cyll end

start $28 \quad 9 \quad 10: 2 \quad 3: 121$ :

$-5.832-2.637-2.48-2.3211 .53511 .6 \quad 0360$

b $111121100000 \mathrm{cyl}$, end end

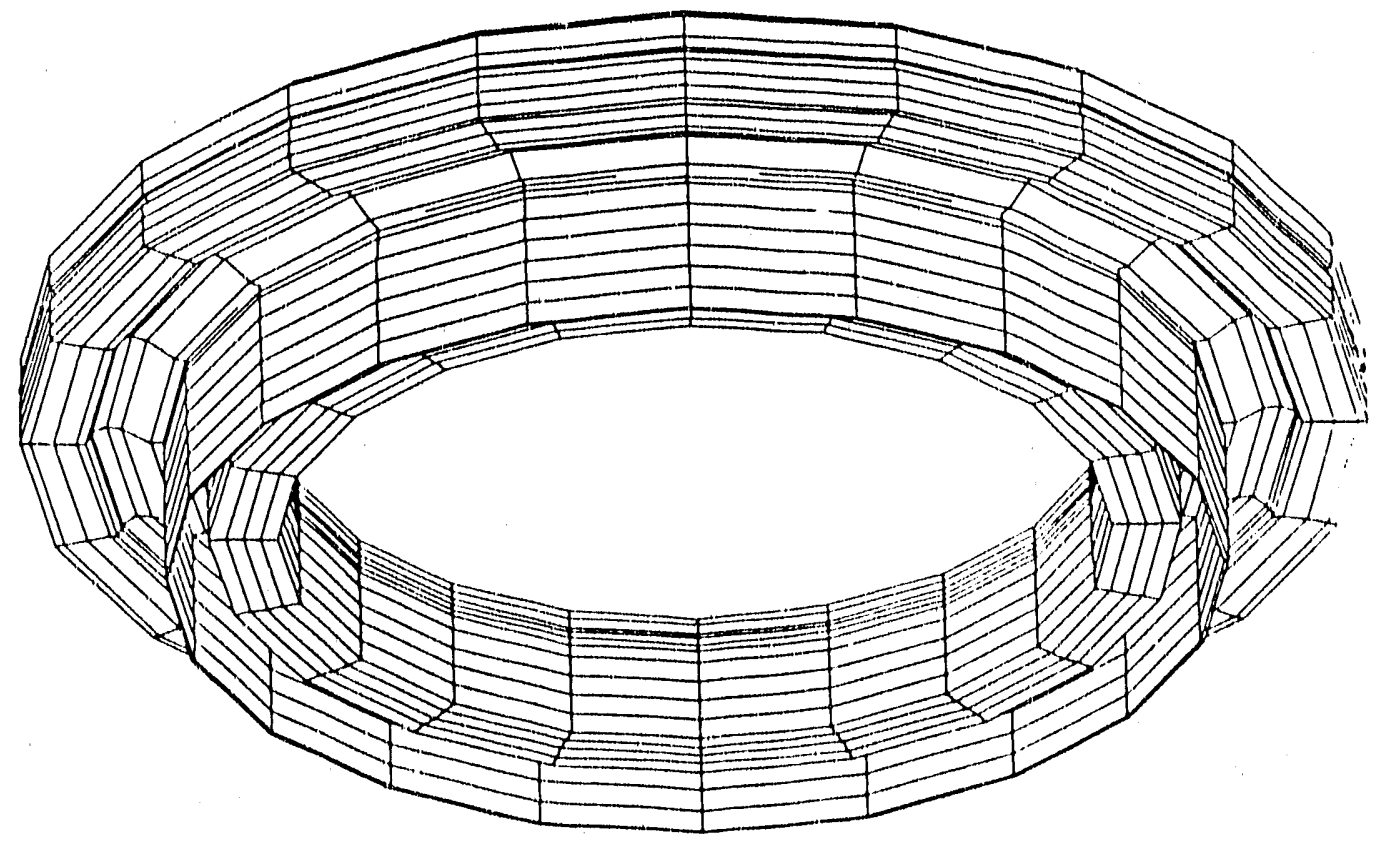


hel ix

dn3d

start

15 :

1226 :

$15:$

$1.52 .50900-.5 .5$

sf: $-1-2 ; 12:-1-2: \operatorname{ts} \theta 00001201$

ecit

fir 11152265

$z=z+0.050 .1$

end

end

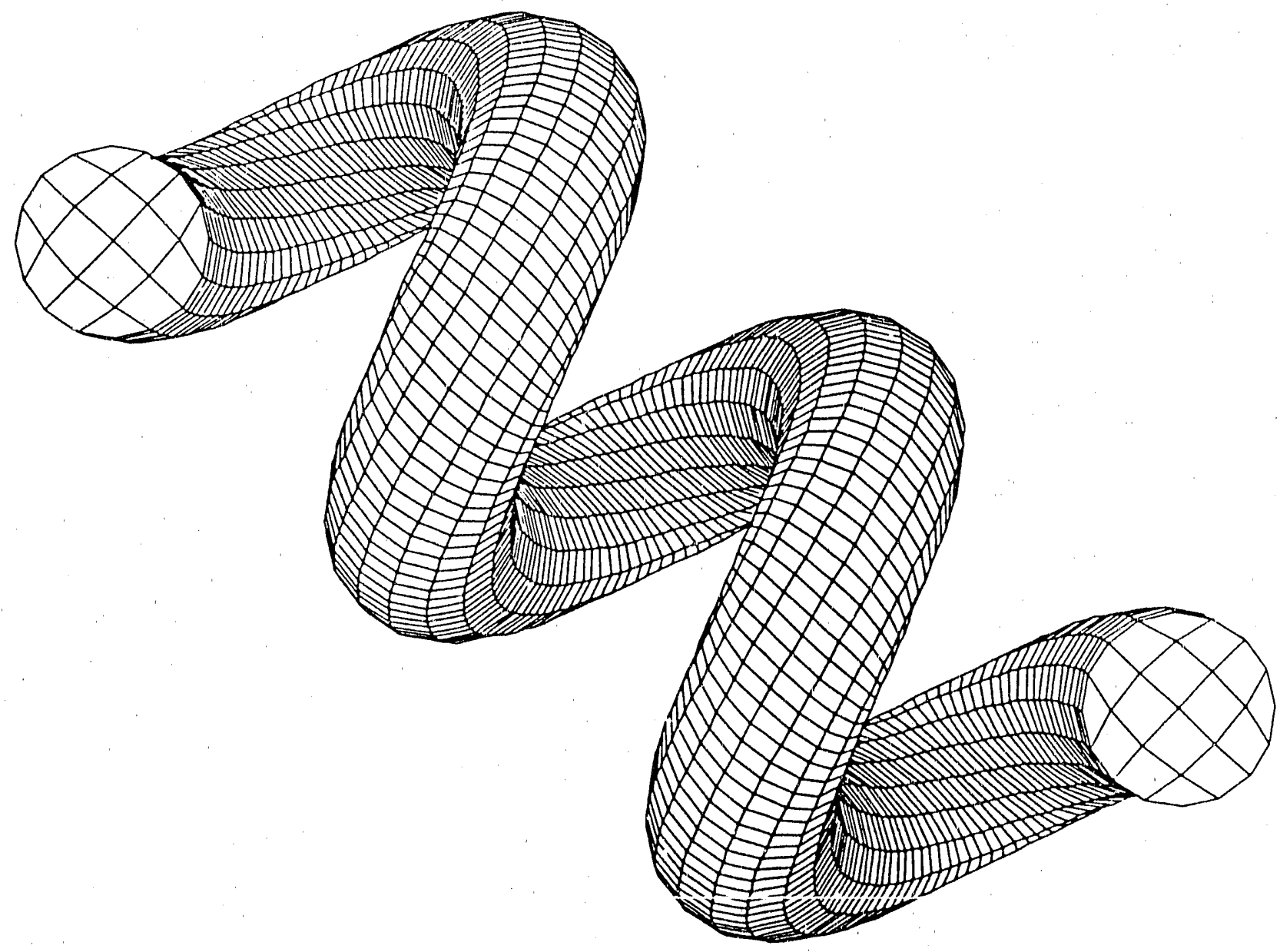


spring

start

15 ;

1331 :

15 :

$1.82 .201320-.2 \quad .2$

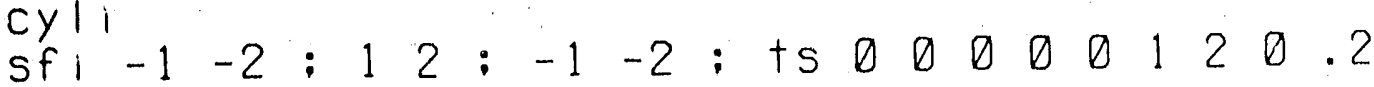
edit

fir 1115795

$z=z+4.05$

fir 18015805

$z=z+4.0625$

fir 18115815

$z=z+4.075$

fir 182152495

$z=z+0.05 \cdot$

fir 1250 i 52505

$z=z+12.475$

fir 1251152515

$z=z+12.4875$

fir 1252153315

$z=z+12.5$

end

end

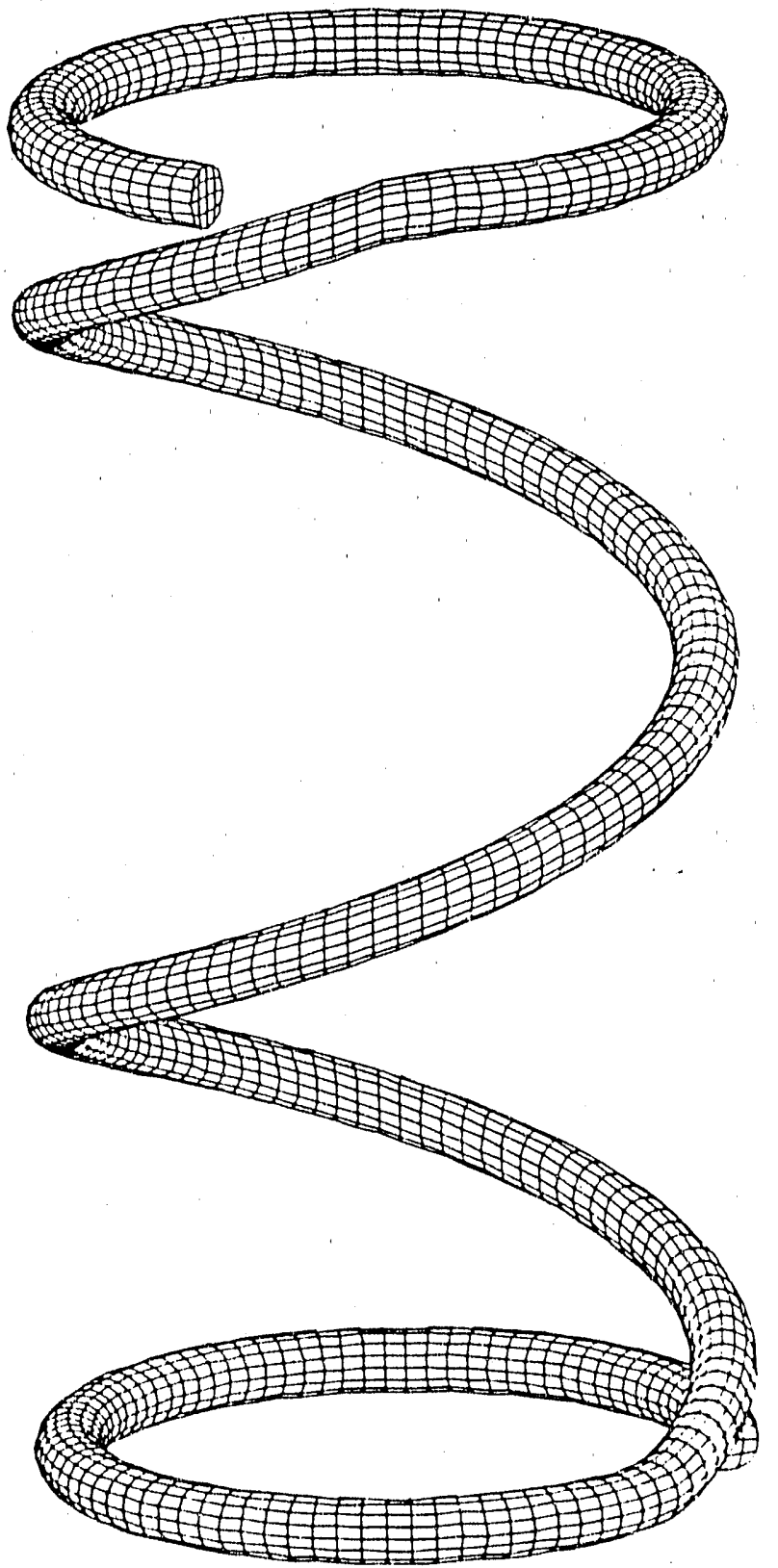


wine glass

$\operatorname{dn} 3 \mathrm{~d}$

start $1915354149 ; 1915354149 ;-1$;

$-1-1-111111-1-1-111110$

di $13046 ; 13046$;

sfi $-1-6 ;-1-6 ;$; cy 000000011.3

sfi $-2-5 ;-2-5 ;$; cy 00000011.03

sfi $-3-4 ;-3-4 ; ;$ cy 00000001.7

edit

cyli

phr $1.00001-600-11.3000016001000$

$\mathrm{z}=2$-sqrt $\left(4-16^{*}(\mathrm{x}-1)^{* * 2}\right)$

end

start $-1 ; 181 ; 140 ; 1.30360 .44$ cyli

edit

cyli

$x=x+(.2 *(k-1) / 39+.15) * \sin (((k-1) / 39) * 405)+.1 *(k-1) / 39$

end

start 1 4;1 4;1 30;-.1.1 -.1.1 $0-2$

sfi $-1-2 ;-1-2 ; 12 ;$ cy 00000001.1

edit

cyli

fir 111445

$\mathrm{x}=\mathrm{x} *(1+.2 *(6-\mathrm{k}))$

fir 11264430

$\mathrm{x}=\mathrm{x} *(1+.2 *(\mathrm{k}-25))$

end

start $161621 ; 161621 ;-1$;

$-.6-.6 .6 .6-.6-.6 .6 .6-2$

di $12034 ; 12034$;

sfi $-1-4 ;-1-4 ;$; cy 00000011.2

sfi $-2-3 ;-2-3 ; ;$ cy 0000001.6

end end

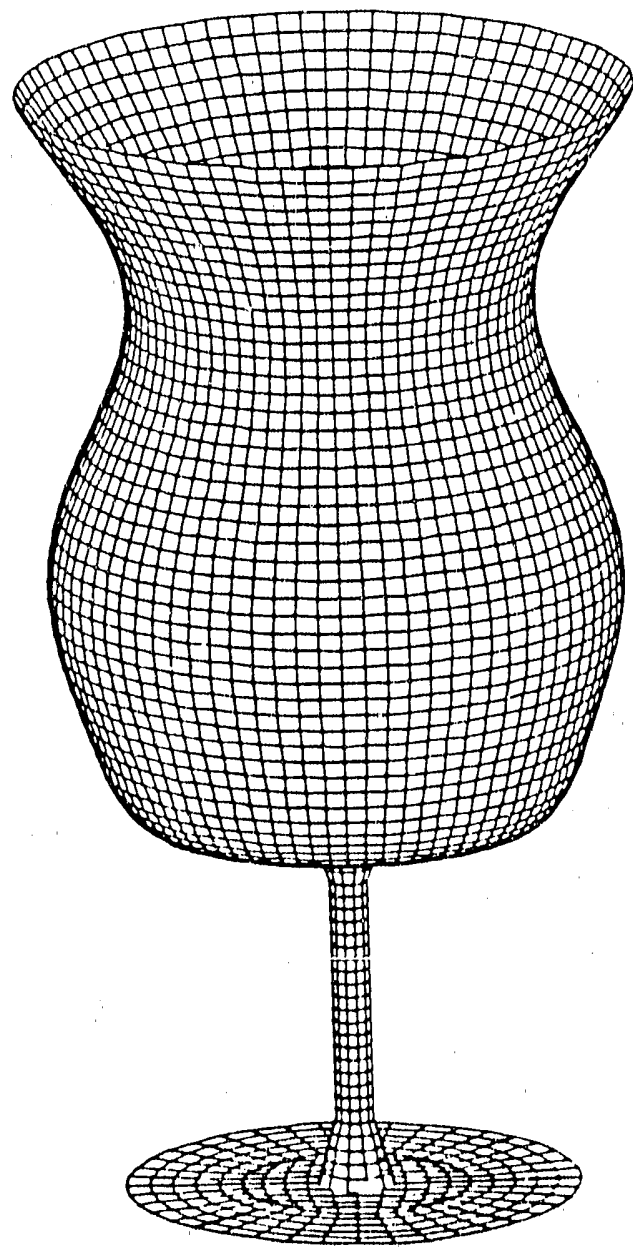




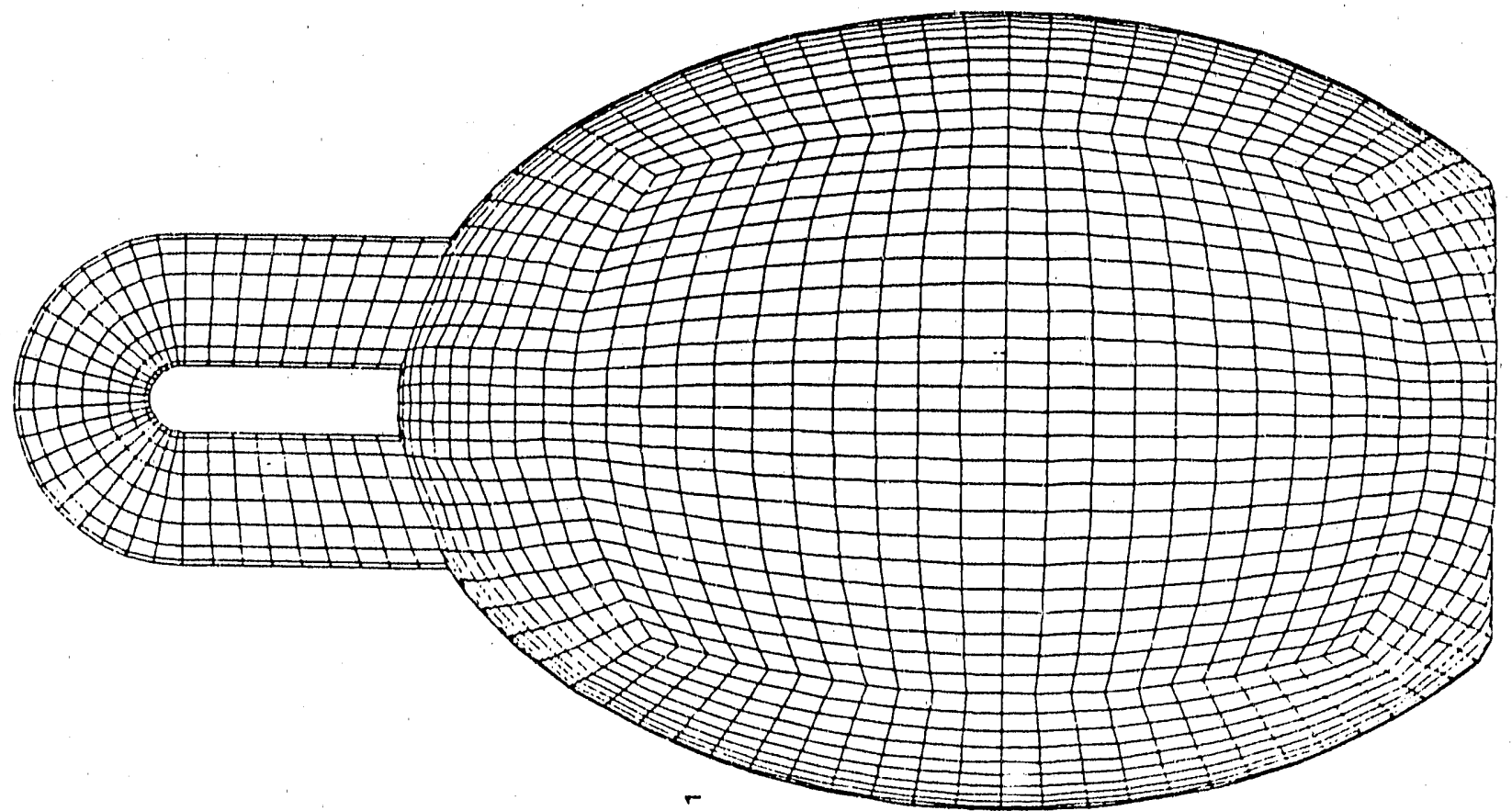

$\ddot{\sim}$

$\mp$

N

$a^{2}$

.100

$\ddot{M}^{T}$ ON

î

$\begin{array}{ll}1+2 & 000\end{array}$

io

"it? nN

กิT

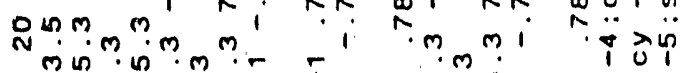
कm

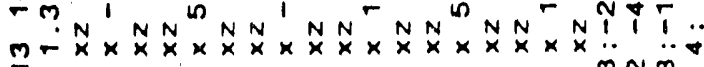

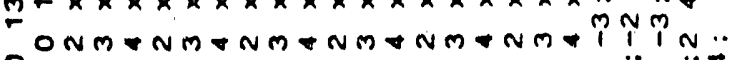
- Oommmmmmommmo-

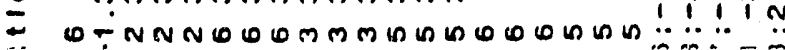
D

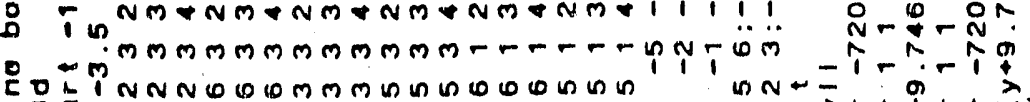

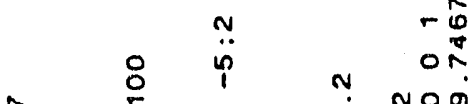

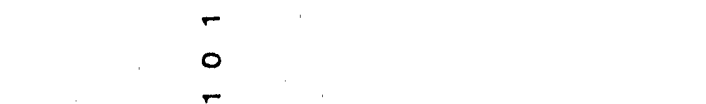

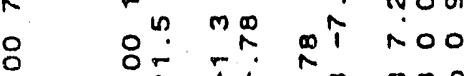
$\rightarrow$ r. ․ NMNNO

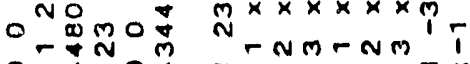

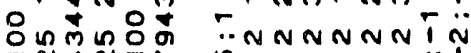

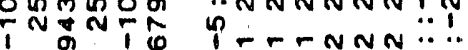
T$=T-\hat{0}-\hat{T}$

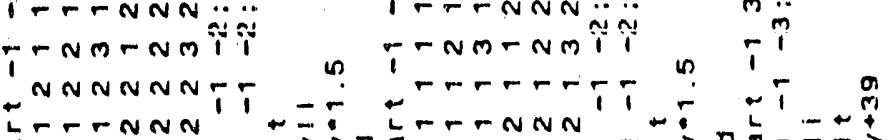

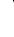
$\infty$ 2

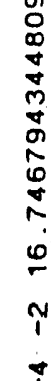
$+\frac{1}{i}$ $\ddot{1}$ No 0 a if Ar: no 0 mo m?mi ?mo ro

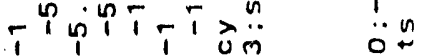
$\because N N N N \because \frac{Y}{O}$ $\ddot{O} \times \times \times \times \times \times M$ OAxaxaxitar - OnM $\ddot{0}-\pi-r-1$ Prr-nNa :.!

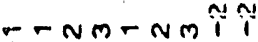
Lr-mnNit :

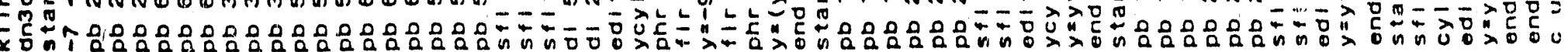




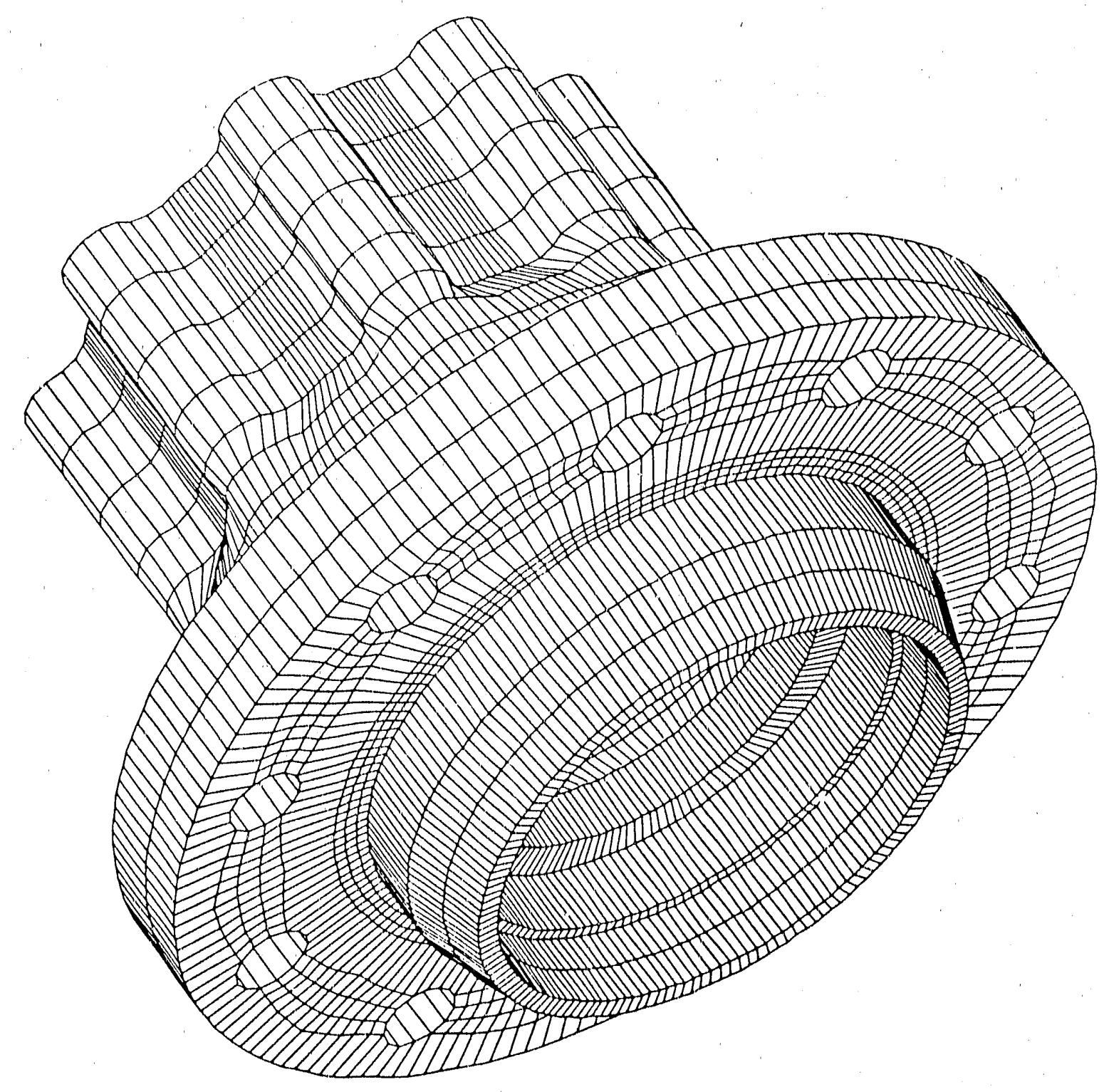


model of rear hub

$\mathrm{dn} 3 \mathrm{~d}$

plan $1 \quad 0 \quad 0001000.001$ symm

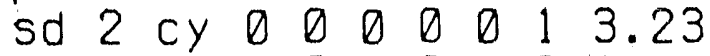

sd 5 cy 0.3 .500 .010 .6349

sd 6 cy $-1.15: 3.6 \quad 0 \quad 0010.562$

sd 8 cy 0.0000012 .375

sd 9 ts 0.05 .81001011 .5902 .0

sd 10 ts 0005.8100010 .2003 .14

sd 11 ts 005.81001012 .1501 .44

coor 1 ryz : lev 1 repe 01 ; : psIv 1

start

$1234781112 ; 139 ; 123457$;

$2.593 .096 \quad 3.2573 .8873 \quad 4.34 \quad 5.053 \quad 6.067 \quad 6.625$

$90.094 .5 \quad 108.05 .816 .447 .25 \quad 7.94 \quad 8.9 \quad 10.0$

pb $6222724 \% 96.0$

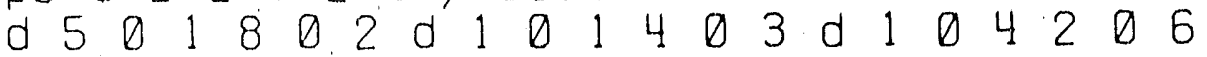

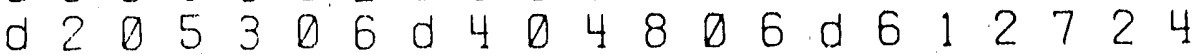

sf $1-6-7 ; 1-2 ; 24 ;$ cy 05.56 0 001.507

sf $1-4: ; 1-3:$ sd 11

pa $406 \times 3.5383$ pa $403 \times 3.4$

ve $61262 \cdot 2+11+k 2+1-1$

ve $622722+k 2$

ve $103103+k 1+11+12+14+k-2+1-3$

ve $205205+11+11+k 1+i-1$

coor 10 rz 0 : rz 36: rz 72 ; rz 108; rz 144:

ryzrz 36 ; ryz rz.72; ryzrz108; ryzrz 144; ryzrz 180: Irep $1 \begin{array}{lllllllllll} & 3 & 4 & 5 & 6 & 7 & 8 & 9 & 10 & \end{array}$

cyli end

start

$12356 ; 14711 ; 14569$;

$2.375 \quad 2.865 \quad 3.2265 \quad 3.7734 \quad 4.1349$

$90.096 .75 \quad 103.5 \quad 112.5 \quad 0.2 .75 \quad 3.444 \quad 3.9495 .81$

d 101012030010430503011422

ma $524 y+2.5$ ma $534 y+1.0$

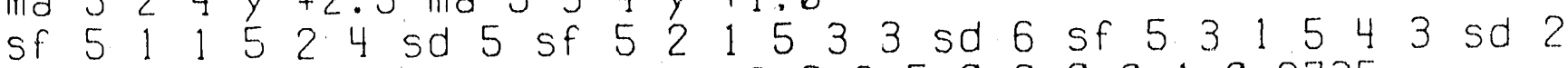

sf $1-3-4 ; 1-2 ; 12 ; c y 0.03 .50 .000 .10 .2735$

sf 1003104 sd 8

sf $1-3 ;:-45$; sd 9

sf $5 \begin{array}{llllllll} & 3 & 5 & 4 & 5 & \text { sd } & 10\end{array}$

sf 505505 sd 10

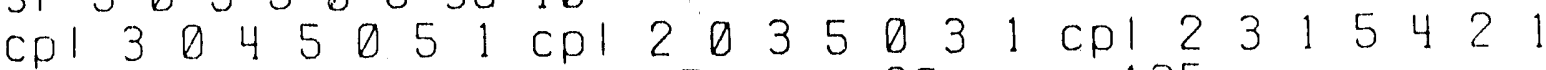

cyl, coor $8 r z 0 ; r z 45 ; r z 90 ; r z 135$ :

ryzrz 45 ; ryzrz 90: ryzrz135: ryzrzi80;

Irep $12 \begin{array}{llllllll:} & 3 & 4 & 5 & 6 & 7 & 8 & \text { : }\end{array}$

ve $311321+k 1+11+k-1$

ve $321421+k 1$

ve $501501+1-3+k 1+1-1+k 1$

end ppiv end 
Dartlally excavated tunnel and nolghbor ing faul t

nk3d anal tat bwmo off nbsry nbol 1 nibsr $10 \mathrm{~ms}+15$ detol.001 octol.01 iprt 1000 Ipit 1 nstop 100 torm .001 c st - minimum 2 coordinato: s/1co 1

- 2 z coordinato for boginning of tunitel plug: s/lco 2

c s3 z coordinate for boginning of tunnol : slice 3

c 84 - 2 coordinato for tunnel opening: sllco 4

c k2 - maximum nodo number of roglon bohina tunnel

c k3 node numbor at sllco 3

c K4 - node numbor at silco 4

c K5 a node number at silco 4 for outar rogion

c rkt - ratlo zoning In $k$ direction In unexcavated rogion

c rke - ratio zoning in $k$ diroction in oxoavatod roglon

c para k2 2 k3 $2 k 43 k 53$ s2 1 s3 2 s4 3 rky . 3 rk2 1.25 :

parak2 11 k3 15 k4 $25 k 514$ s2 50 s3 60 s4 65 rk1 .8 rk2 1.25

mat 15 mat 25 ondmat

sd 1 cy 011.5000011 .5 sd 2 cy 0000.0016 .5 sd 3 plan 300100

sd 4 plan 1.062 .5600100051 tlod:sl 2 sv:

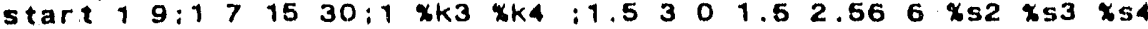

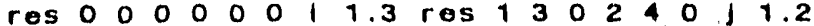

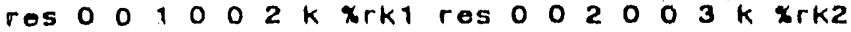

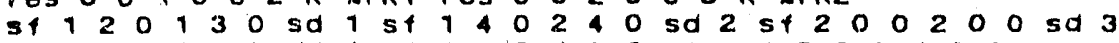

sf 1300140 sd 4 sf 1100120 pian 1.500010

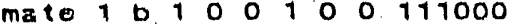

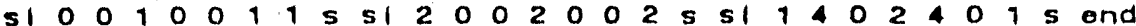

start $116: 120: 1 \times k 3 \times k 4: 36.545$ 152.3 *s2 xs3 \%s4

mate 1 cyli

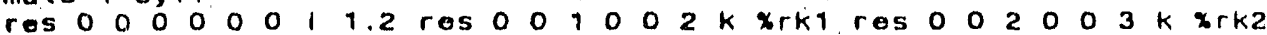

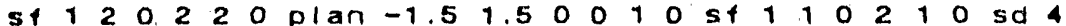

st 11100120 sd 1 st 21012220 sd 2

si $210022015 s 1001000115$ and

start 1 16:1 $7: 1$ *k3 *k4:-6.5 $-1.5001 .5 \times 52 \times s 3 \times s 4$

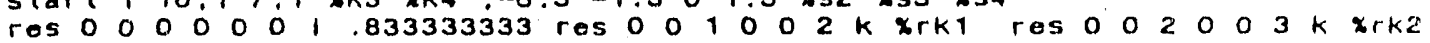

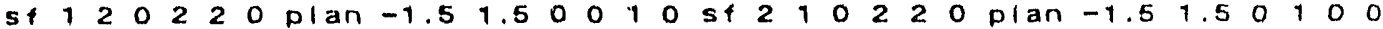

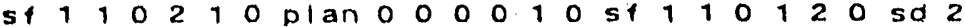

mate 1 b 1000100111000

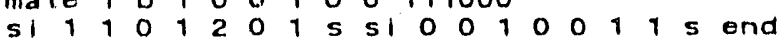

start $1508: 1 \quad 7 \quad 12$ 19 $22: 1 \times k 3 \times k 4: 34.74 .7 \quad 0 \quad 1.5 \quad 2.564 .54 .5 \times 52 \times 53 \times 54$

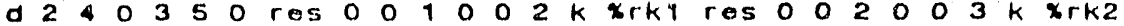

po 230230 y 32.7 pb 340340 y 5.8

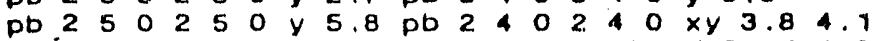

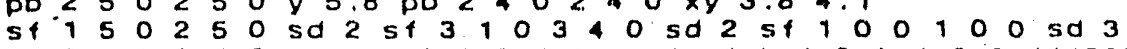

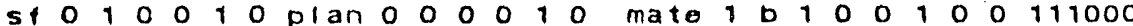

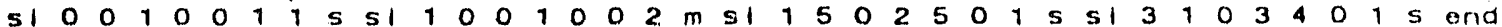
start $1516 \quad 20: 171519: 1 \times k 3$ :

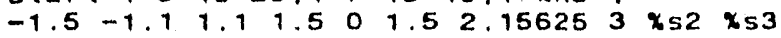

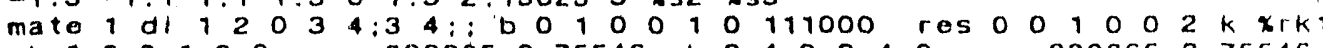

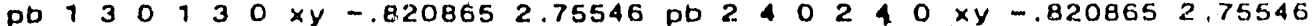

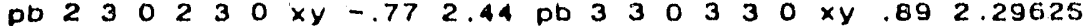

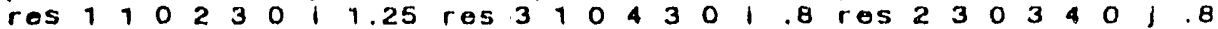

st $2 \begin{array}{lllllllllllllllll}3 & 0 & 3 & 3 & 0 & \text { c. } & 0 & 1.5 & 0 & 0 & 0 & 1 & 1.1\end{array}$

st 3400340 sd 4 st 4300430 sd 4

$5+1-1-4: 2 \quad 3:: 5 d \quad 1$ sf 2400340 sd

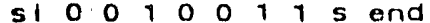

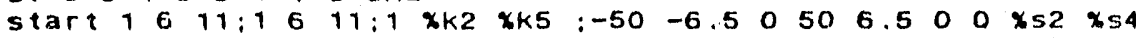

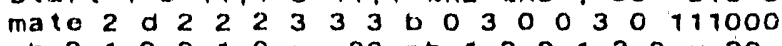

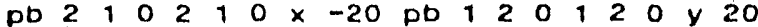

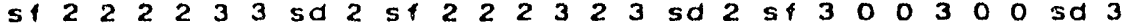

s| $\begin{array}{llllllllllllllllll} & 1 & 2 & 3 & 2 & 3 & 2 & \text { s } & \text { si } & 3 & 1 & 1 & 3 & 3 & 2 & 2 & 5\end{array}$

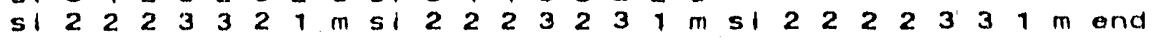

start $19911: 1611: 1 \times k 2 \times k 5: 506.53506 .500 \times 52 \times s 4$

mato 2 d $2 \begin{array}{llllllllllllll}2 & 2 & 3 & 3 & 3 & b & 0 & 3 & 0 & 0 & 3 & 0 & 111000\end{array}$

pb $2100210 \times 13$ pb 12012120 y 20

st $2 \begin{array}{llllllllllllllllllllllllll}2 & 2 & 2 & 3 & 3 & \text { sat } & 2 & \text { st } & 2 & 2 & 2 & 3 & 2 & 3 & \text { sd } & 2 & \text { st } & 3 & 0 & 0 & 3 & 0 & 0 & \text { sd } & 3\end{array}$

$\begin{array}{llllllllllllllllll}\mathrm{s} & 3 & 1 & 2 & 3 & 2 & 3 & 2 & \mathrm{~m} & \mathrm{~s} & \mathbf{3} & 1 & 1 & 3 & 3 & 2 & 2 & \mathrm{~m}\end{array}$

$\begin{array}{llllllllllllllllllllllllllll}s 1 & 2 & 2 & 2 & 3 & 3 & 2 & 1 & m & s & 2 & 2 & 2 & 3 & 2 & 3 & 1 & m & s & 2 & 2 & 2 & 2 & 3 & 3 & 1 & m & \text { and }\end{array}$

end c use stp.001 to mergo parts

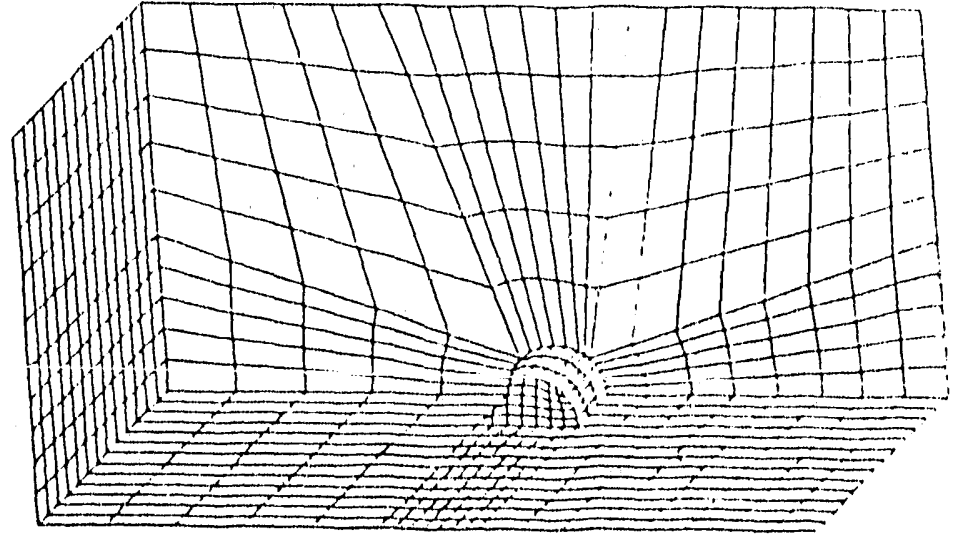


MAZE example in INGRID-see MAZE manual dn $3 d$

plane 3

$\begin{array}{llllllllll}0 & 0 & 0 & 0 & 0 & 1 & .0002 & \text { symm }\end{array}$ 0000010.0002 symm

00010100.0002 symm

si 1 si:

si 2 sl:

10110200200

Id 2 Ip $214 \quad 14 \quad 01414$

lat $028-128 \quad 14$

ld $31 p 200040$

$104 \quad 1 p 20014 \quad 20 \quad 14$

$\begin{array}{lllllllll}\text { Id } & 5 & \text { Ip } & 2 & 18 & 0 & 18 & 14\end{array}$

lep 18.220140900

part 4233311010

spin 18.90

si 111112221110

si $1122222 s$ end

part $12243 \quad 322020$ spin 1890

si 112222215

si 211122225 end

t13 part 15423320 $\operatorname{spin} 1890$

si $11111222 \mathrm{~m}$

end

part $4 \begin{array}{llllll}5 & 3 & 2 & 3 & 9 & 40\end{array}$

spin 1890

si $1111222 \mathrm{~m}$

end

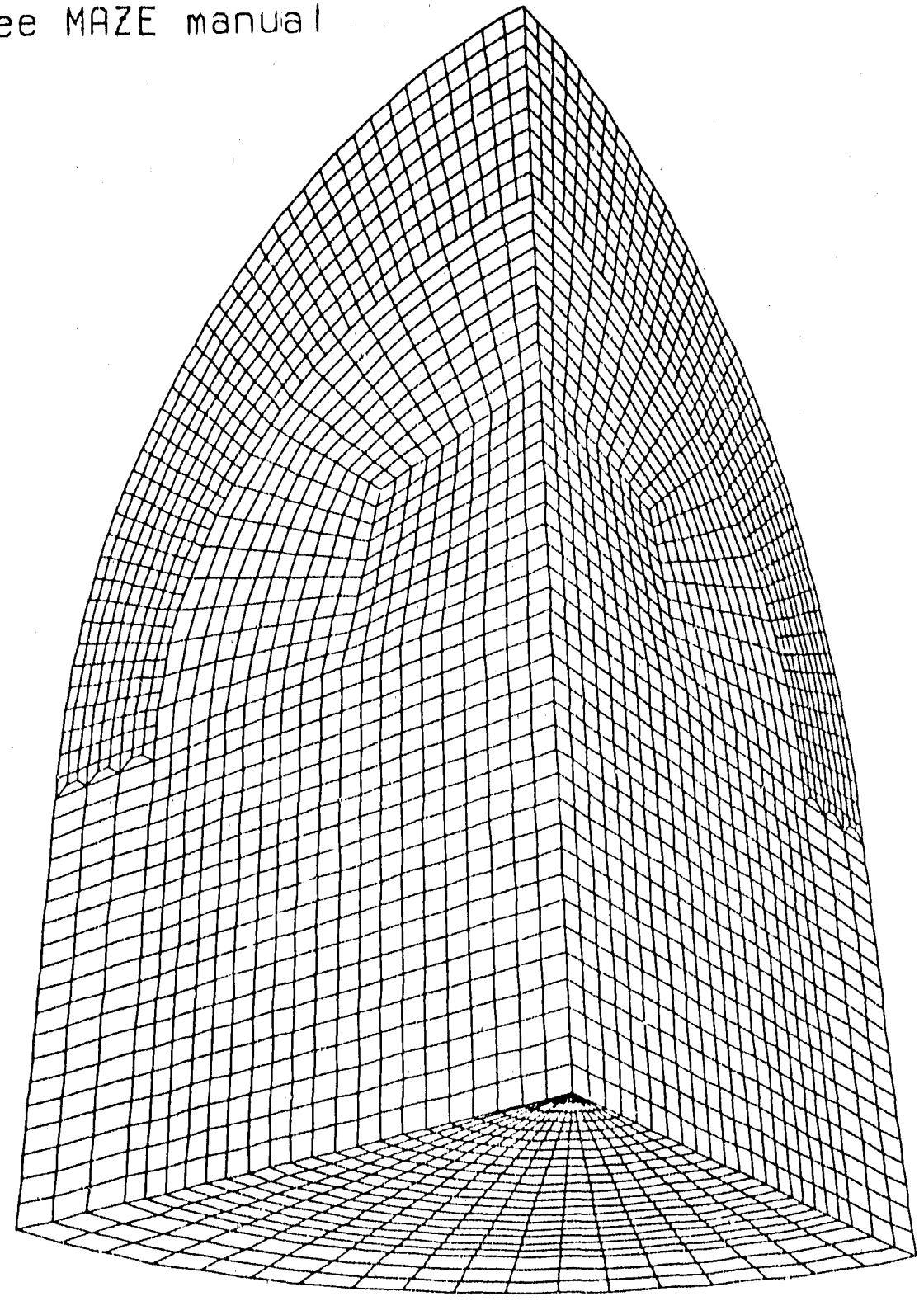

mat 11 heading

elastic mater al 6061-t6 aluminum

.ro 2.71 e .689 pr .33 hgqt 2 hga .15

endmat

mat 23 heading

elatic-pastic mater ial 1020 steel

ro 7.83 e 2.07 pr .29 sigy .004

beta 1.0 etan .010 endmat

mat 33 heading

elastic material 110 copper

ro 8.94 e 1.17 pr .33 hgq .05

endmat

end

c use stp 0.002 in the interact!ve

c stage to merge parts 3 and 4 

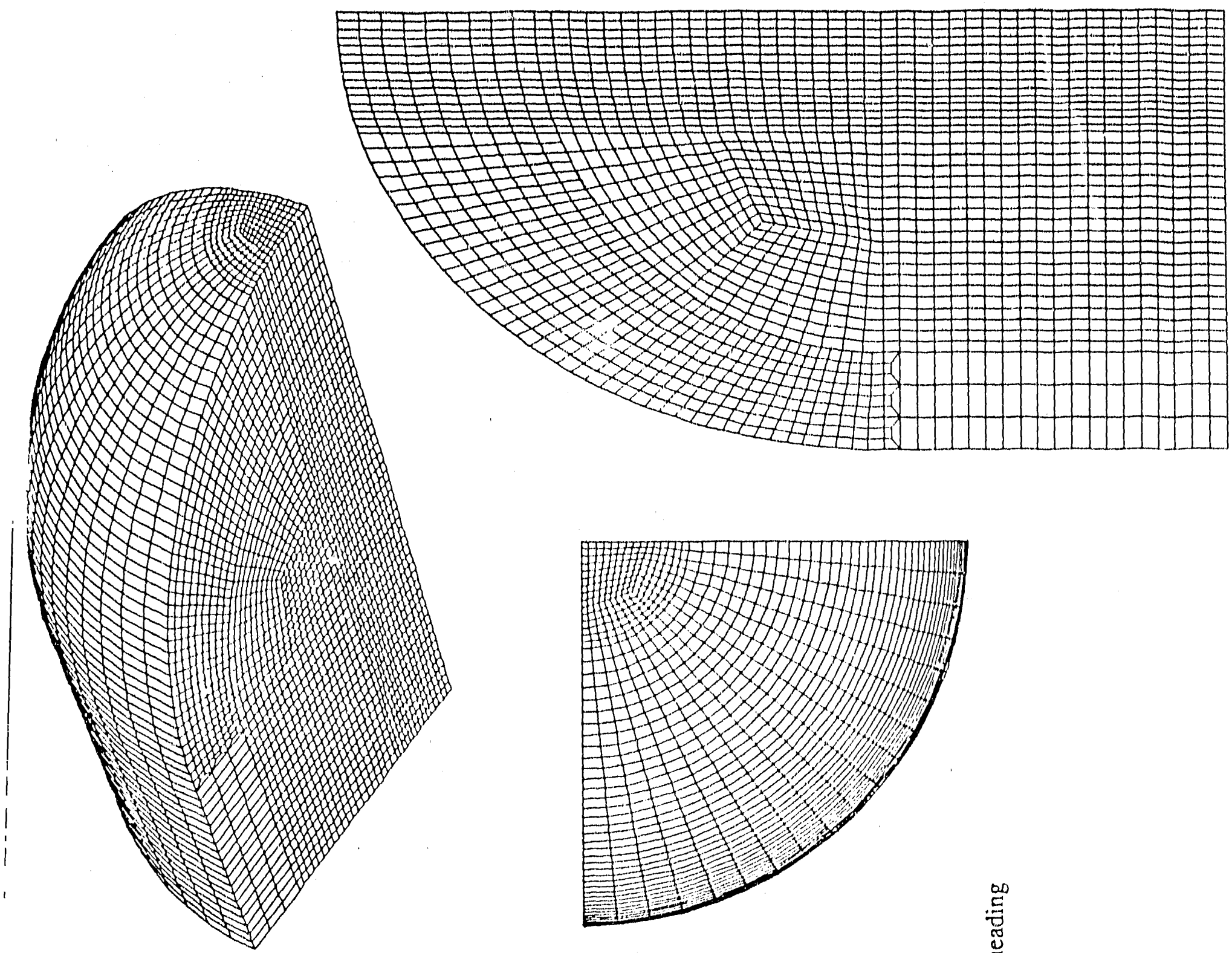

莺

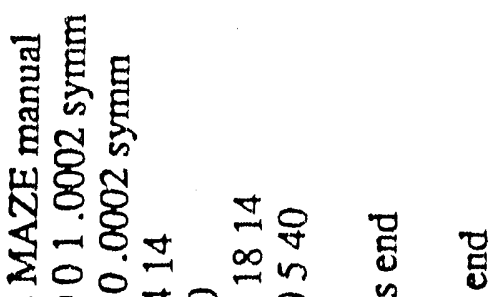

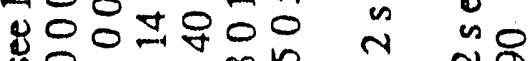

mono

ió

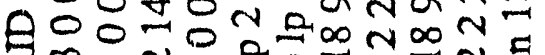

व

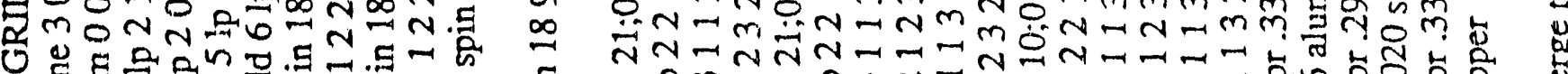

Z

.

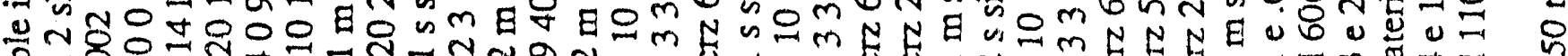

ใ.

जٓ

J-T -

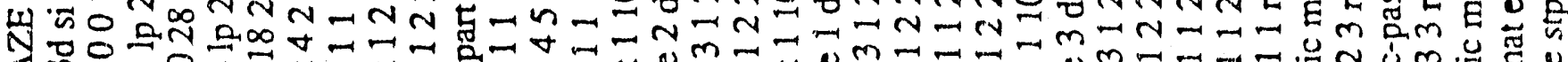

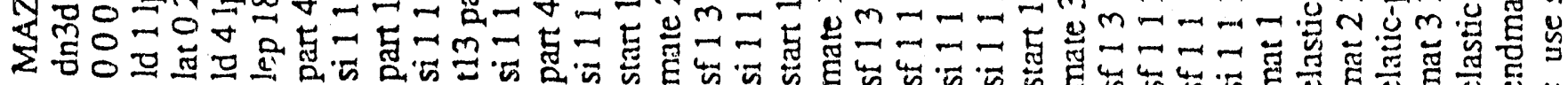




\section{INGRID/DYNA3D/TAURUS ROTATING PIPE EXAMPLE}

This simple test problem contains 1 part. It has a constant pressure load on the inside of the pipe pointing outwards. It is rotating. The following is the input to INGRID. The ingrido file from INGRID is used as the input to DYNA3D.

rotating pipe

dn3d term 2.5e-03 plti 1.25e-05 prti 2.5e-03

mat 13 e $7.1 \mathrm{e}+10 \mathrm{pr} .333$ sigy $2.86 \mathrm{e}+08$

etan $3.56 e+09$ beta 0 ro 2680 endmat

Icd $1206.69 \mathrm{e}+0710006.69 \mathrm{e}+07$

start 12;1 73;1 11;

.02413 .0266703600 .02413

cyli mate 1

rotation 000004000

epb 111111 ;

pr 10010011100180.013

end end c use stp .00000001 
The following is the interactive input to TAURUS after this program has initialized. The period is the prompt from TAURUS.

\author{
.angle 1 \\ $. r \times 30$ \\ .state $1 \mathrm{~V}$ \\ .phs2 \\ .elements 11 \\ gather \\ .etime 811 \\ etime 111 \\ .etime 211 \\ .end
}




\section{rotating pipe \\ time. $\theta$.}

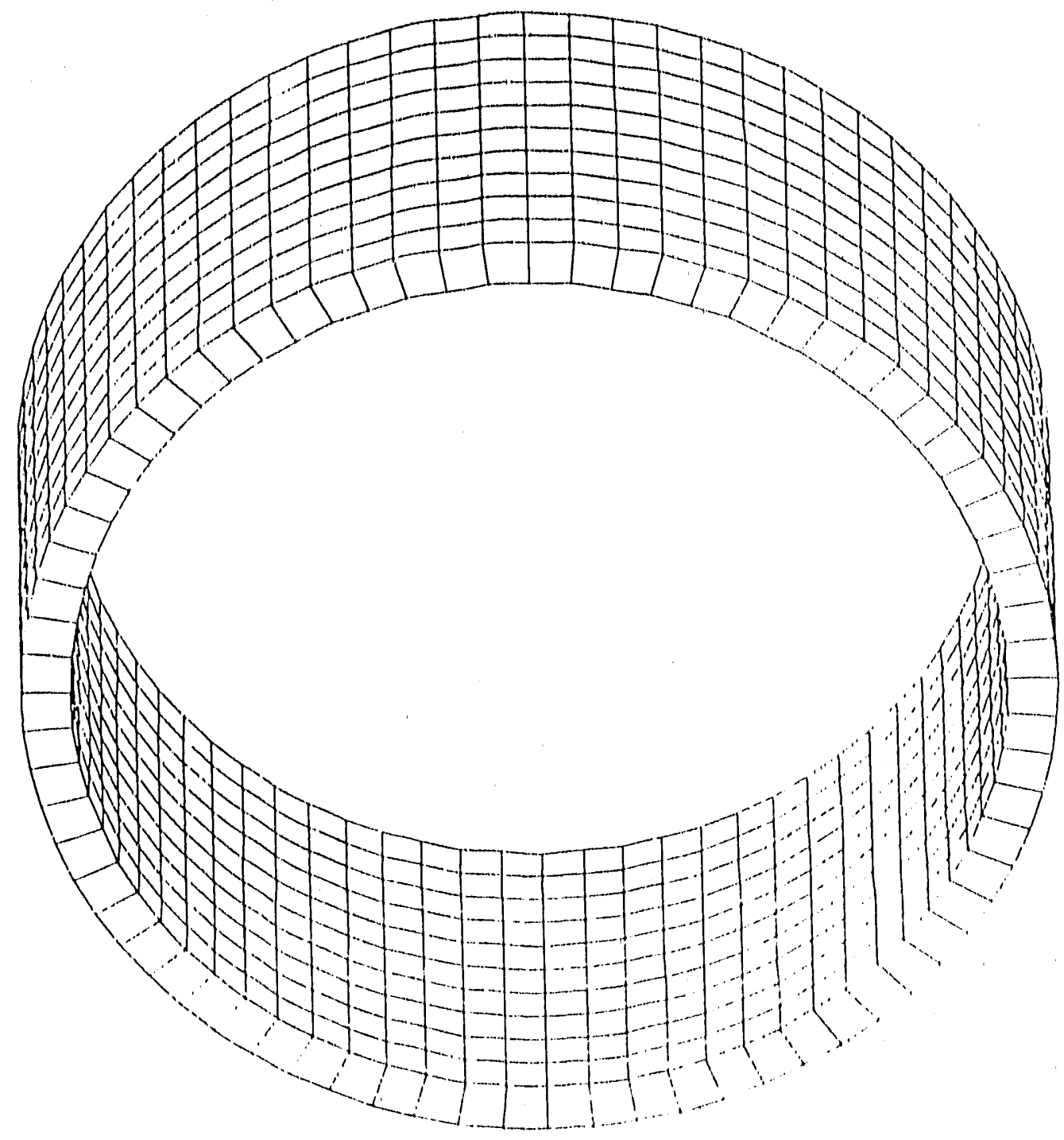




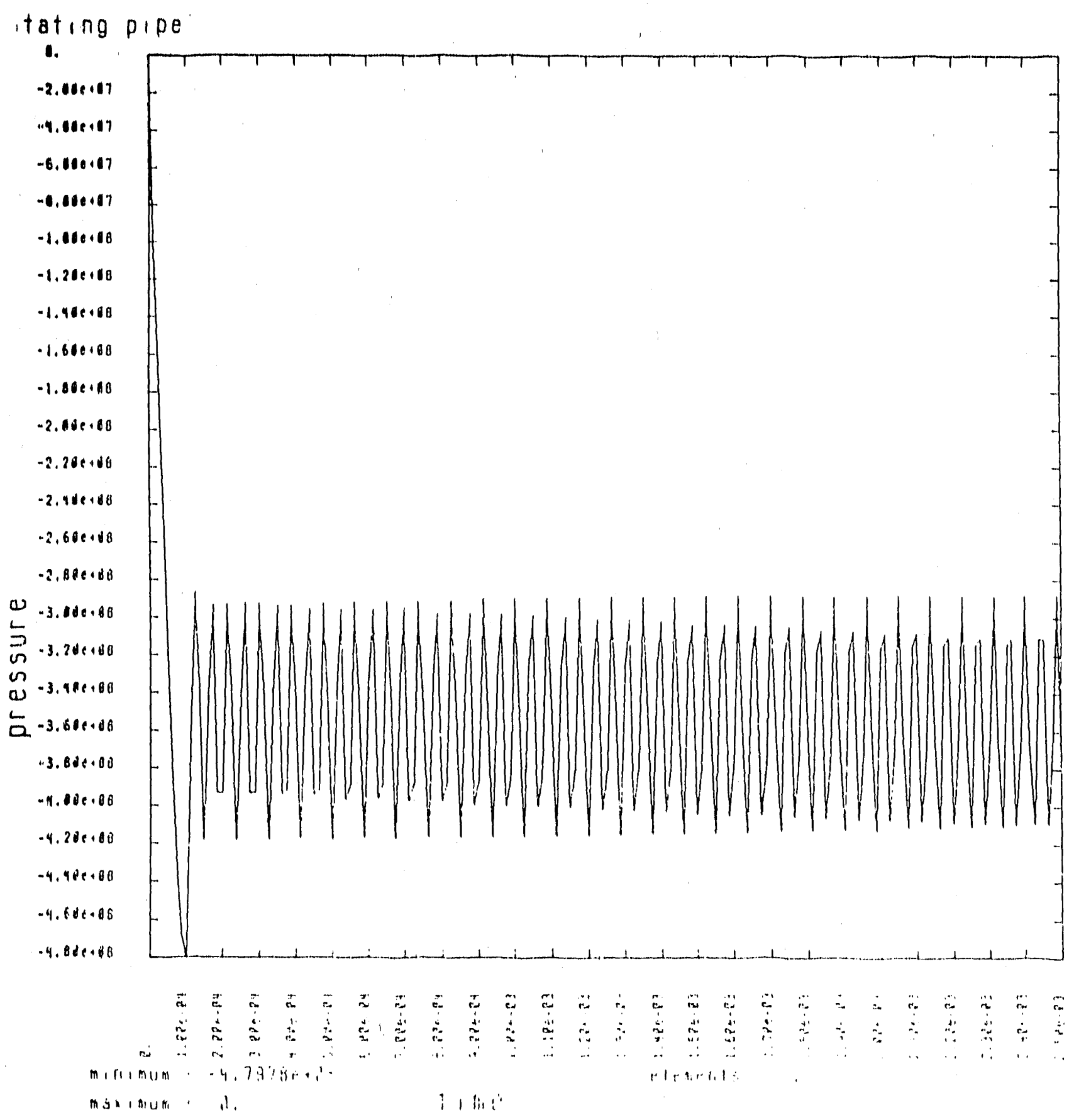




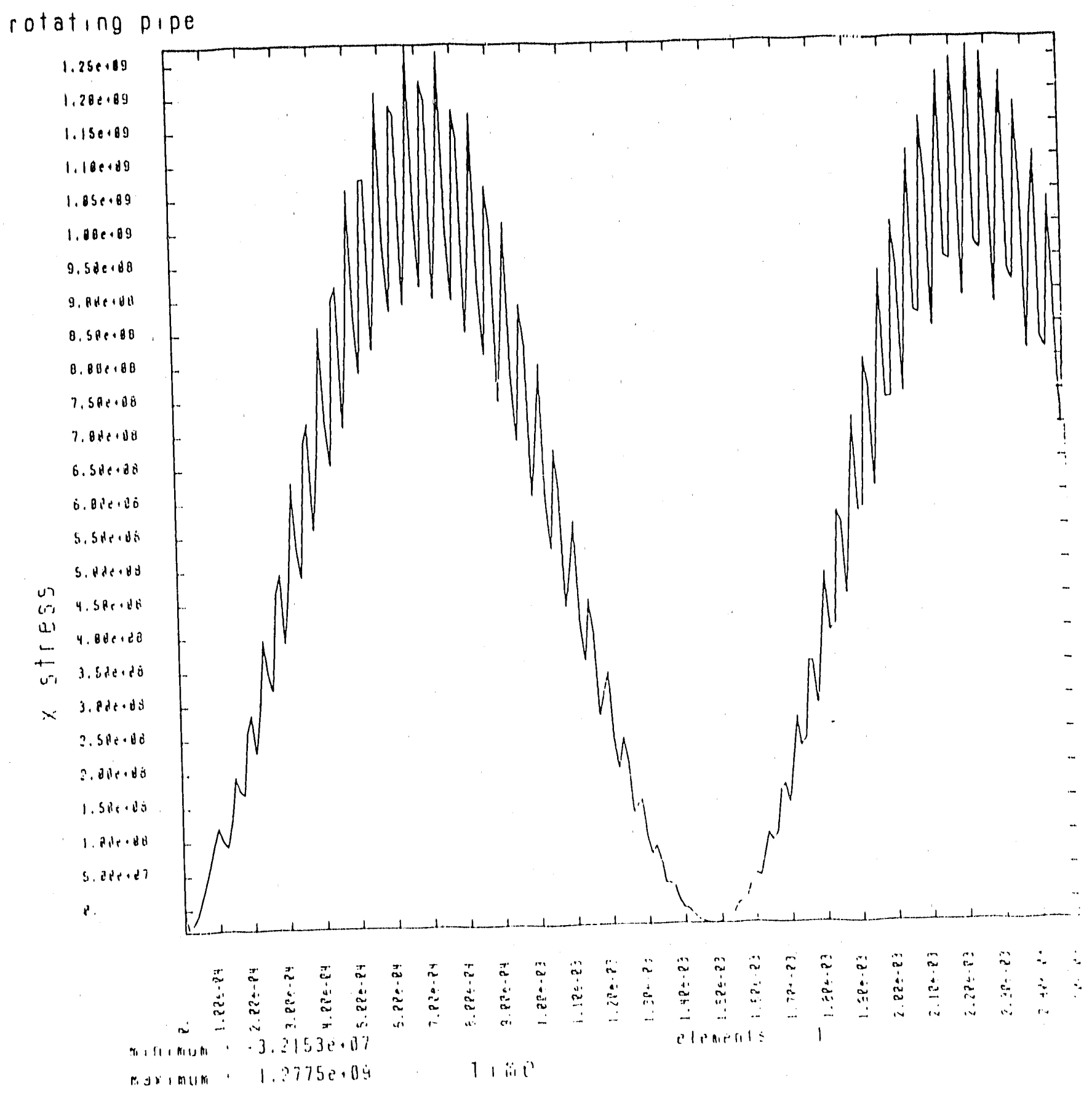




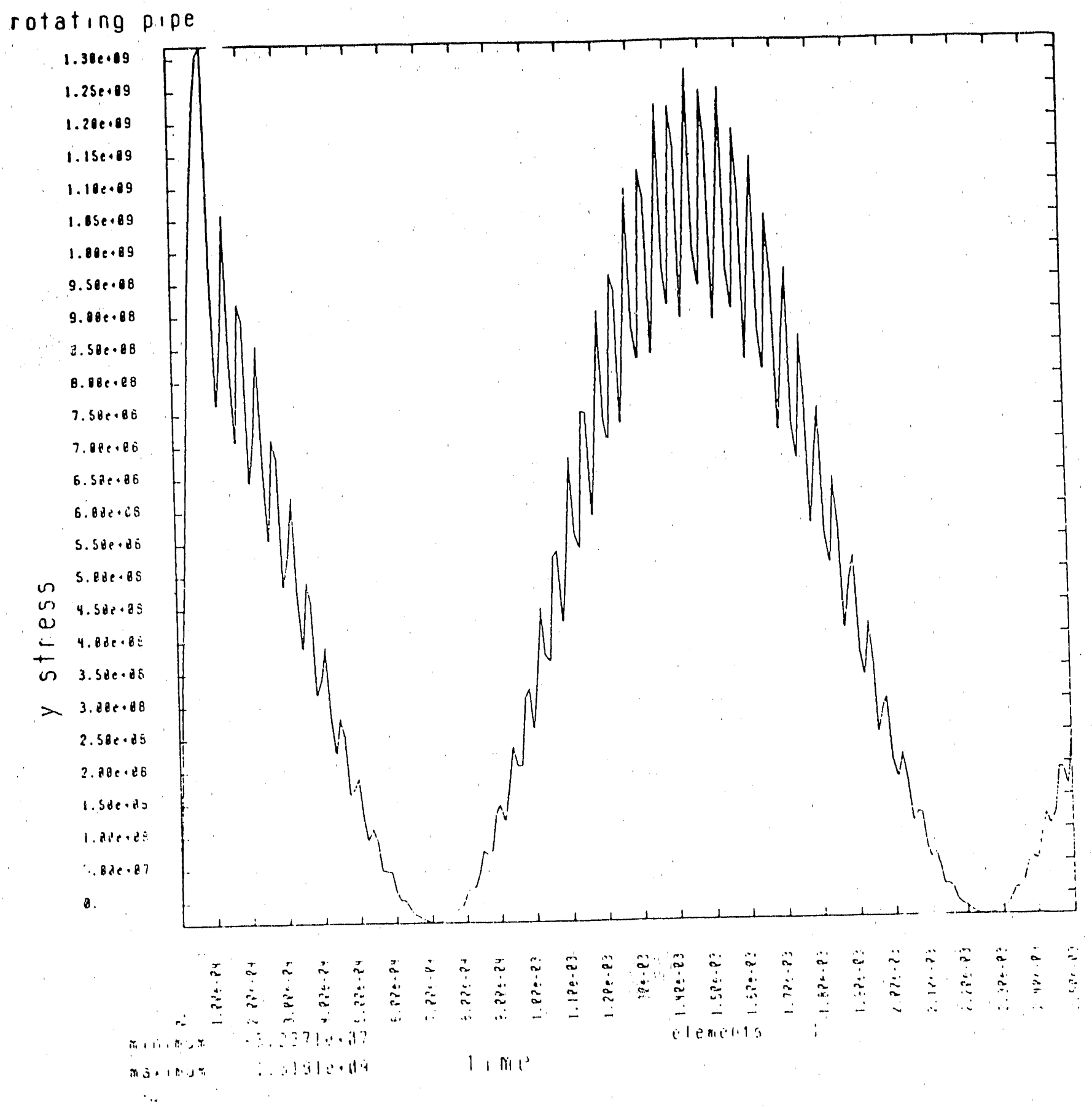




\section{INGRID/DYNA3D/TAURUS IMPACTING BAR EXAMPLE}

The problem is calculated with $1 / 4$ of the model using 2 planes of symmetry. A third plane of symmetry is used to simmulate the bar impacting it's reflection.

Note that the quarter bar is generated where 2 logically distinct faces of the mesh are placed in the same location. When INGRID is in the interactive mode, use the command

stp .001 continue

to connect these two faces and write the input file for DYNA3D. 

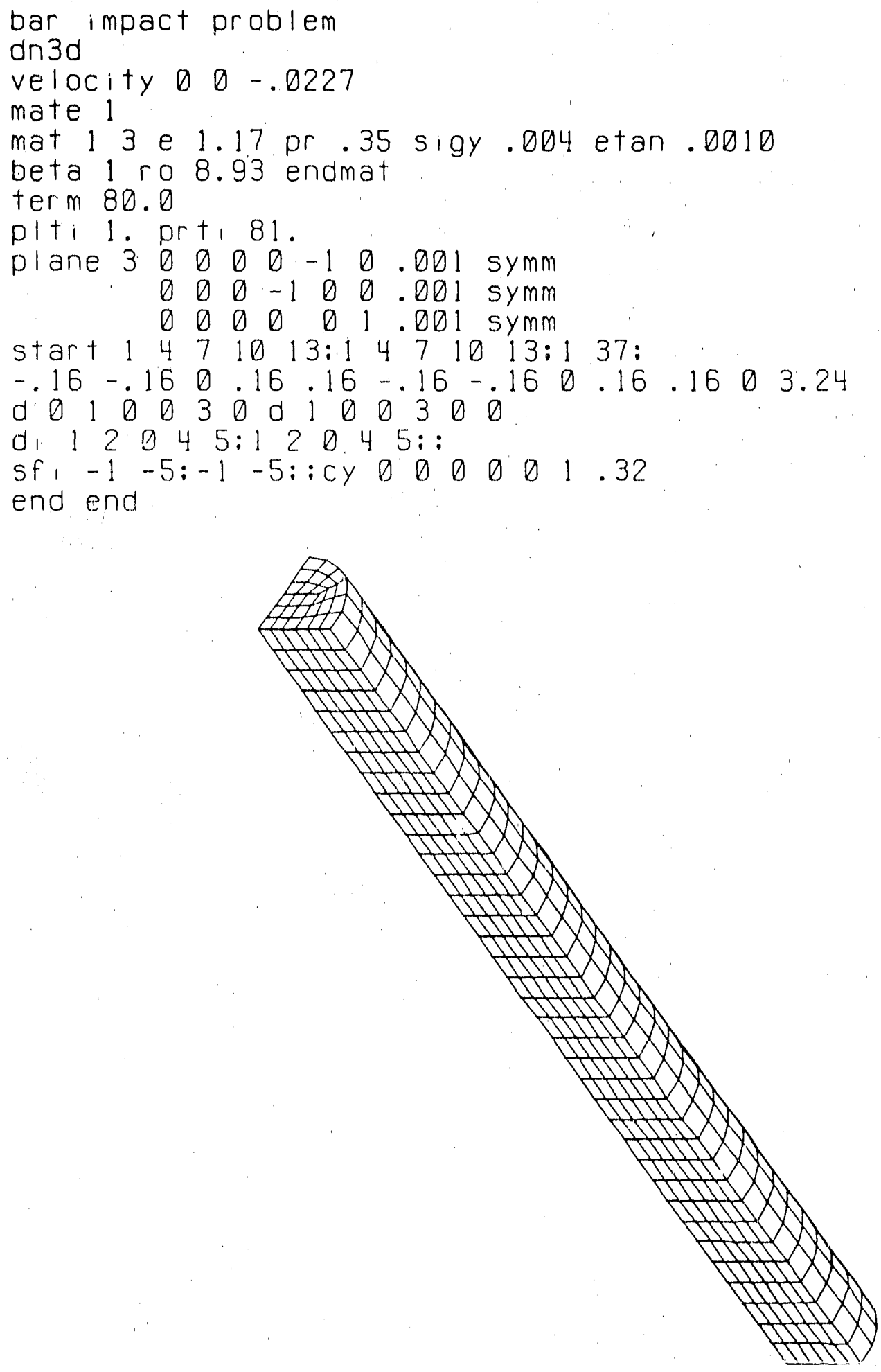
The following is the interactive input to TAURUS after

TAURUS was initialized with the plot files from the DYNA3D run. The period is the prompt from TAURUS for more input.

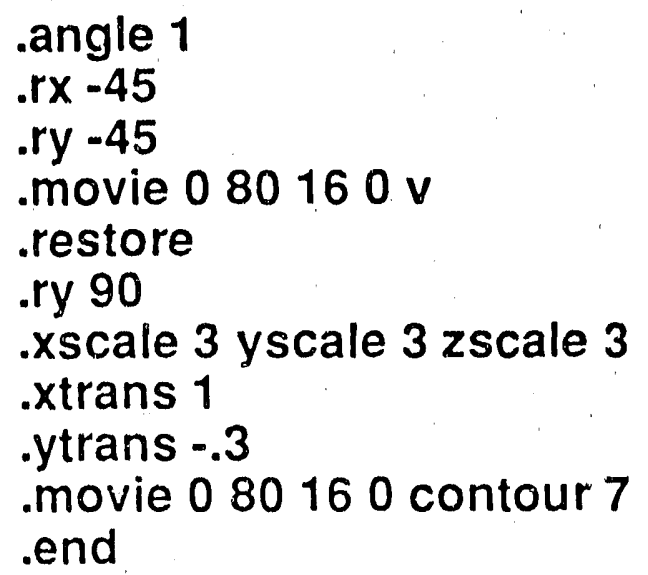


ca. aso grodem

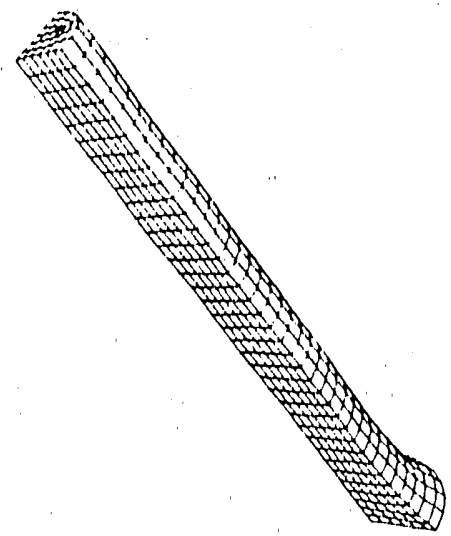

Dar impact problem

1.me. $1.50000 \mathrm{e}+01$
Dar impact problem

lime. 1.00000e.01 bar impact prodem

1.me $2.00000 \mathrm{e}+8$
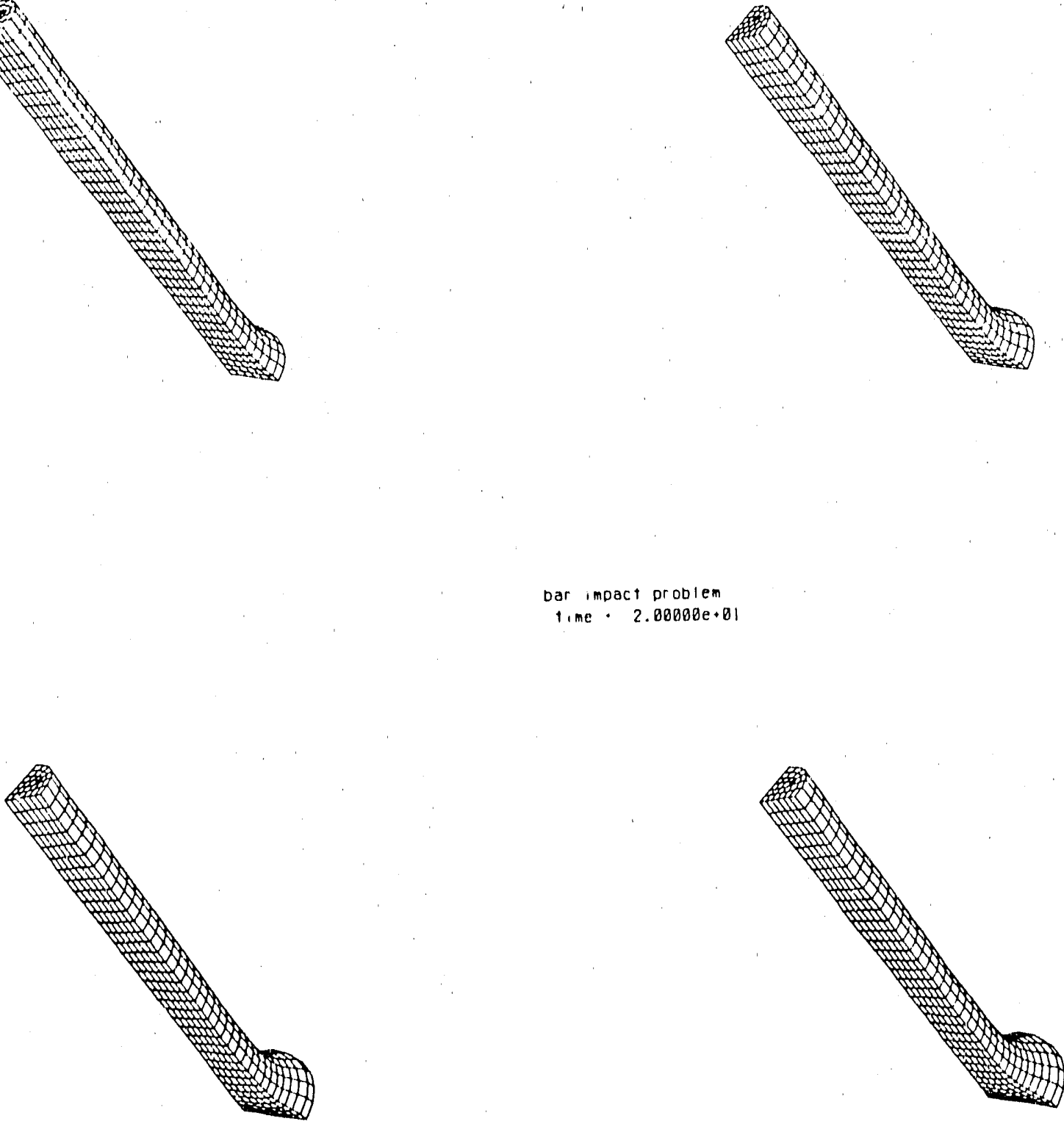
bar impact problem

time $2.500000+01$

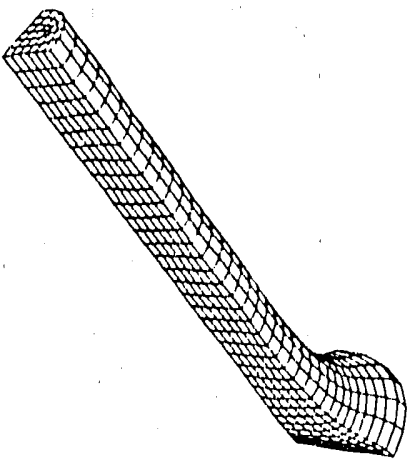

Dar impact proder.

time. $3.50023 e \cdot 3$ ! bar impact problem

lime. $3.80000 \mathrm{e}+\theta$

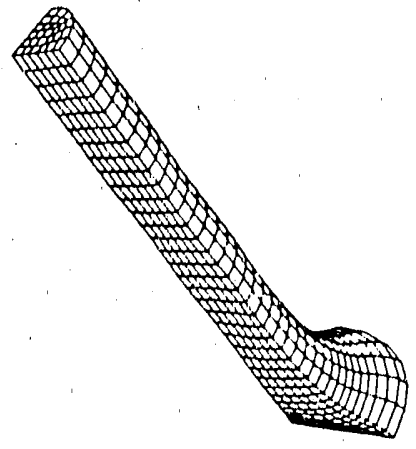

Dar impact problem

time $4.08088 \mathrm{e}, 81$

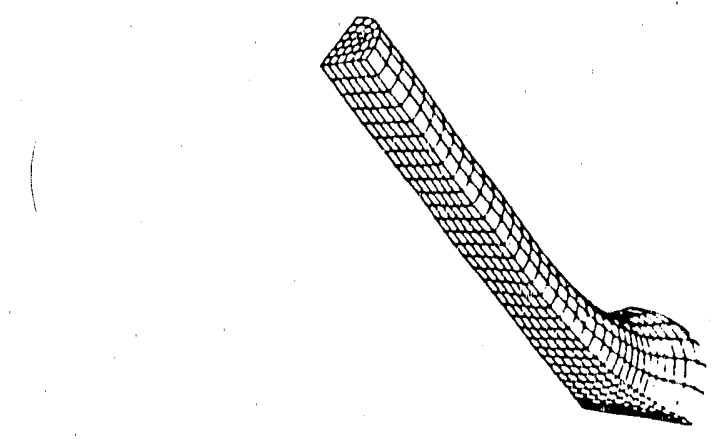

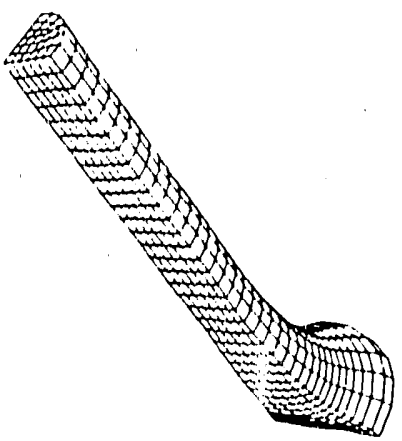




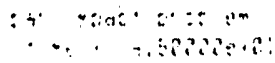

bar mpact problem
time. 5.gogebe+01
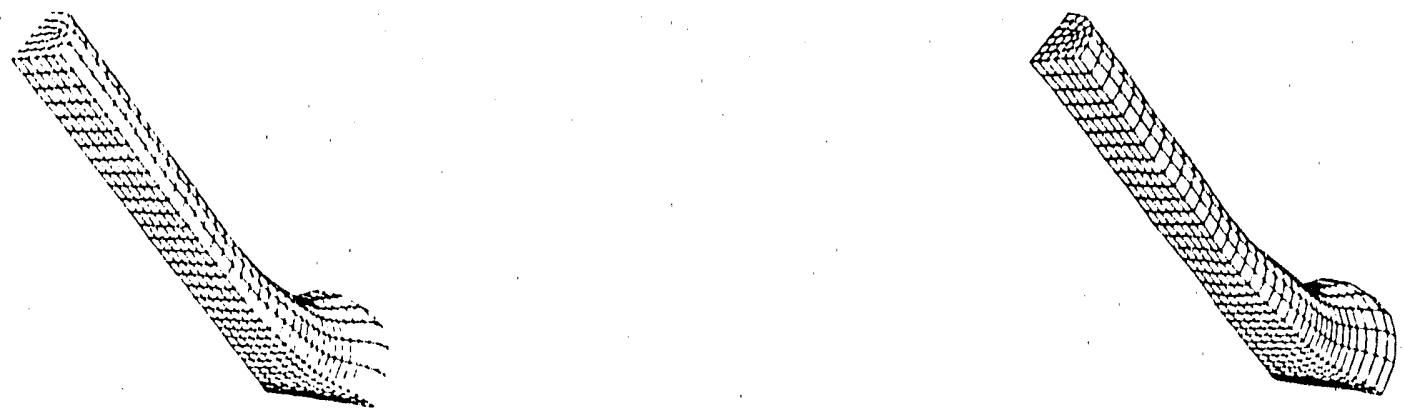

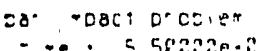

Dar impact problem

time - 6. Bopobe+81
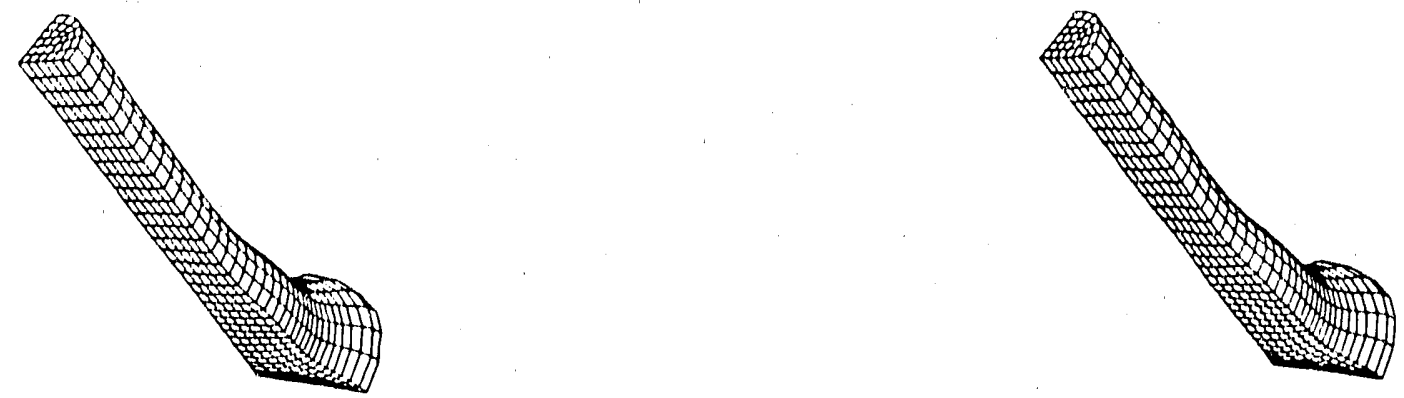
Dar impact problem

time. $6.500080+81$ ber impoct problem

time $7.00000 \mathrm{e}+81$
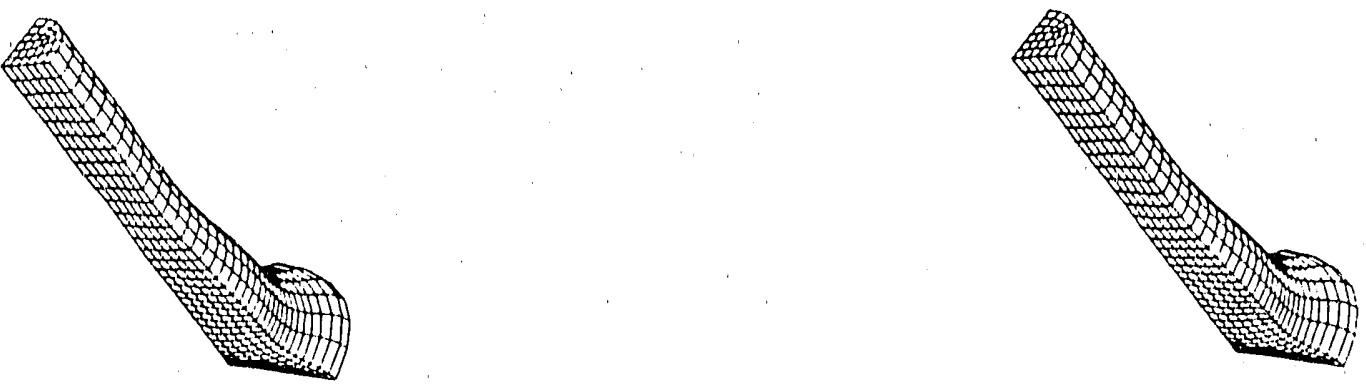

Dar impact prodem

i. me $0.00000 \mathrm{e} .81$ bar impact proolem

time - $7.50038 \mathrm{e}+01$
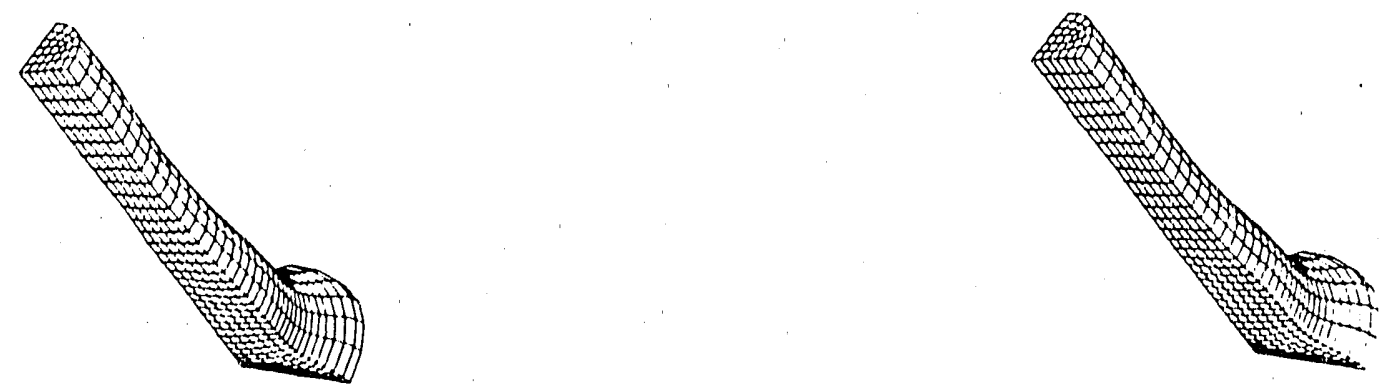


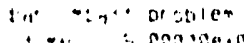

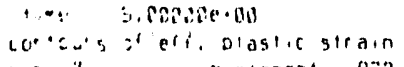

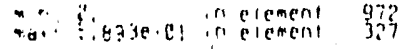

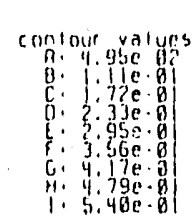

Dar impas ? urablem

time. 1.98006e.61

contours of efr. plastic stran

min: 9.9000 .91 in element $97 \%$
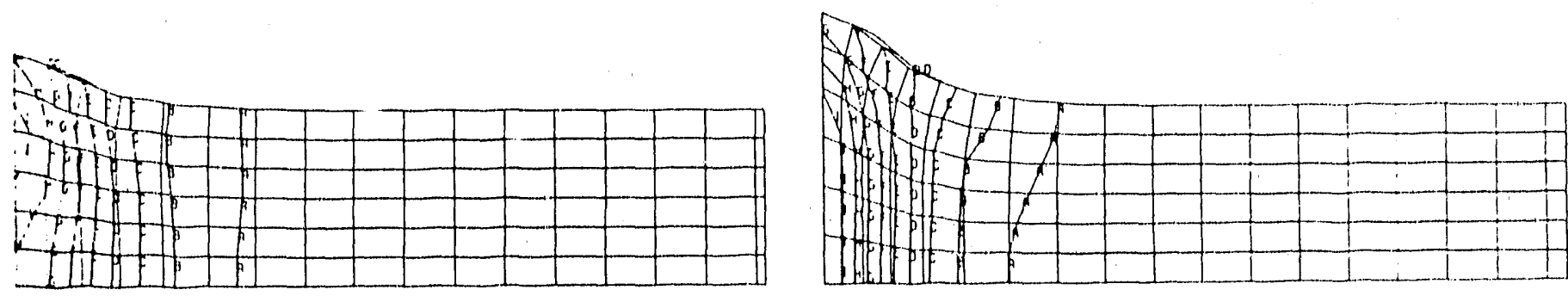

Dar ipcact prodem

1.me 1.50080e.01

conicles of eff. plastic strain

mox. i.46le.00 in element 972

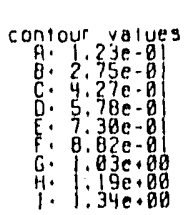

oar impact problem

time - 2,00008le.gl

contours of eff. plastic sirain

in: 0 .

C. $3.51 \mathrm{e}-\mathrm{e}$

$9.34 \mathrm{e}-0$

i. $1.32 \mathrm{sec}$
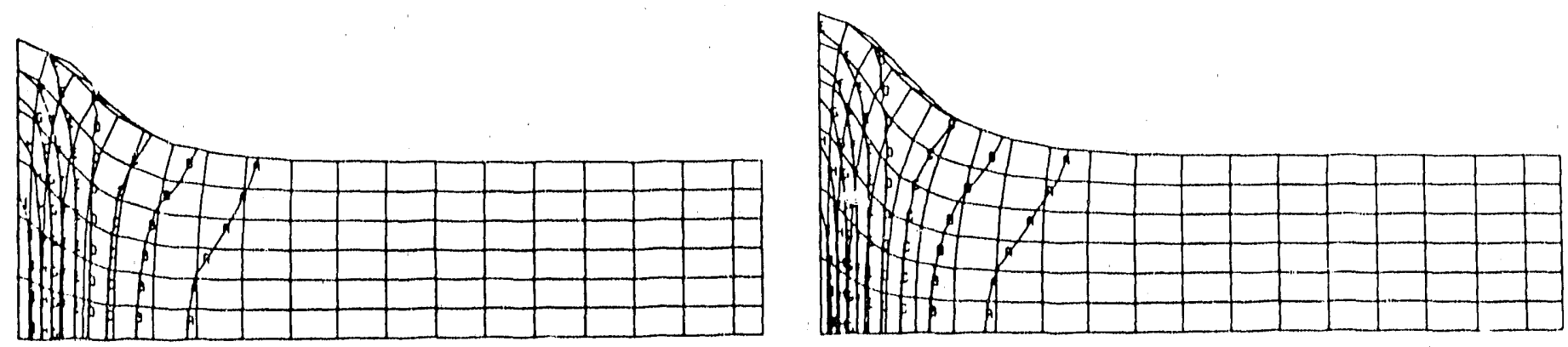
tor impact problem

liwe. 2,50000e+01

contours of eff. plaslic strain

max: 2:281e+00 in element 97$\}$

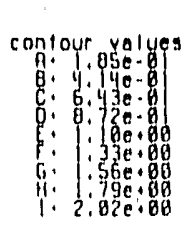

bar impact problem

time $3.000000+\theta 1$

contours of uff, plastic steain

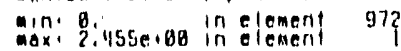

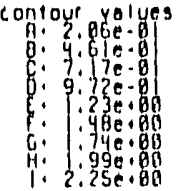

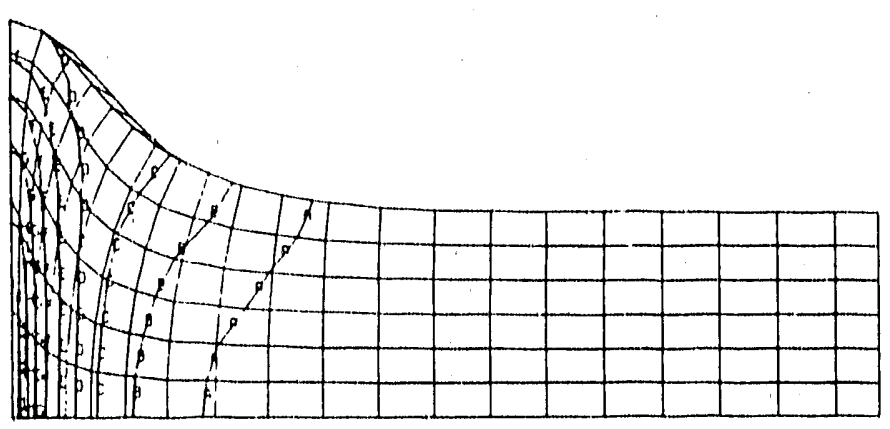

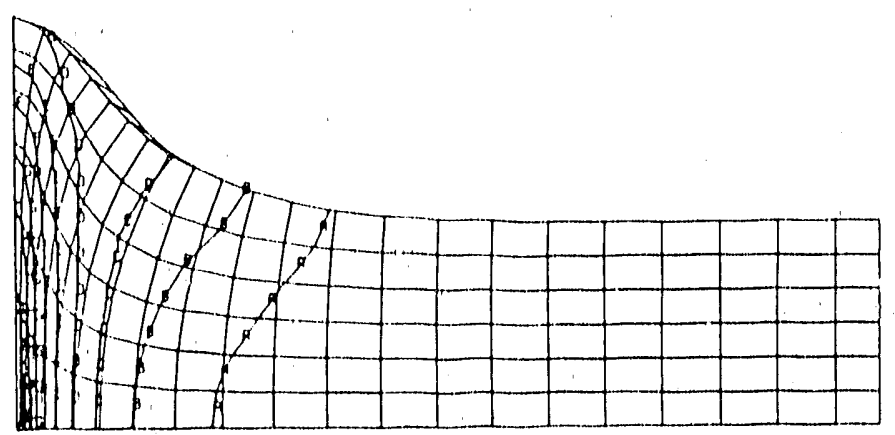

Do imDast prodiem

lime 3.58zaze-3i

contours of eff, dostic strain

man. R.632e.02 in element 972

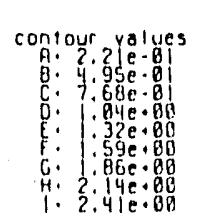

bar impact problem

1ime. 4.08083e.01

coritours of ell. plastic sirain.

min. gi.742e+80 in element 972

H. $2.14 e \cdot 00$
1. $2.41 \mathrm{e} \cdot 00$
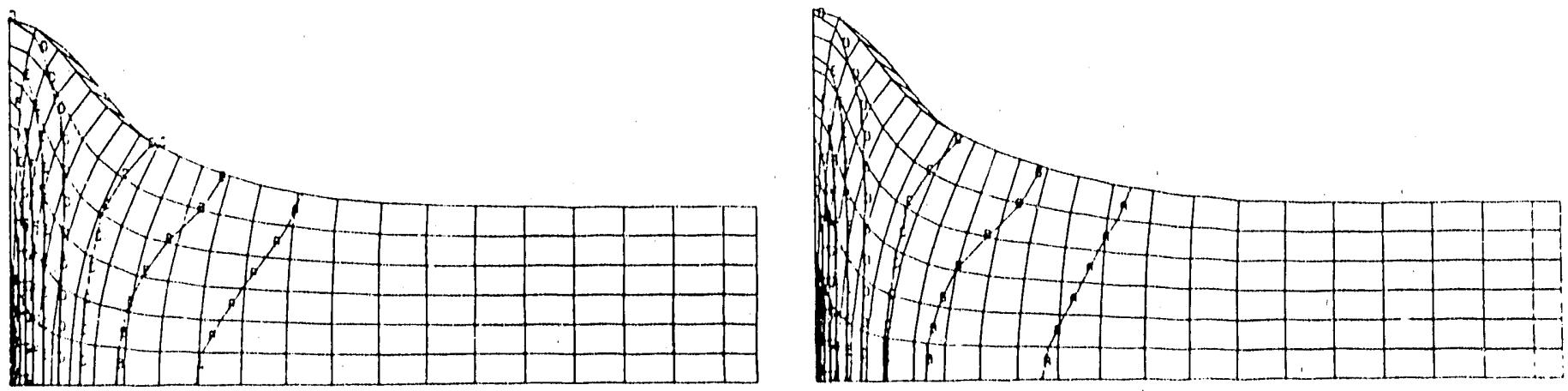
ra. moact broblem

-ine. 4.500ide.01

conlours of elf, blagtic sirain

mav. D. rgseras in element 972

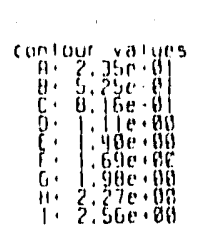

bat imfac: 1 problem

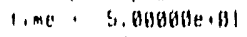

conlours of eff. plaslic sleain

man: B. Boyerog in element 97?
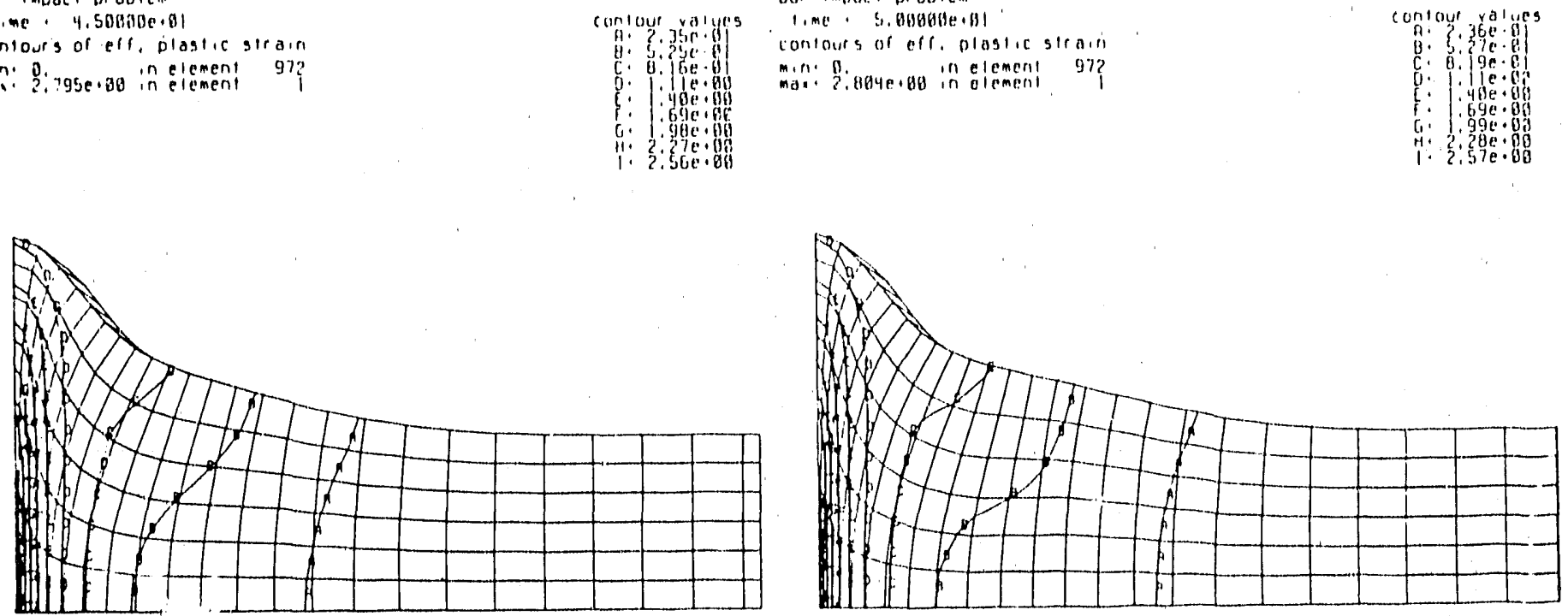

our impoct problem

tontourg of eff. plostic strain

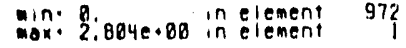

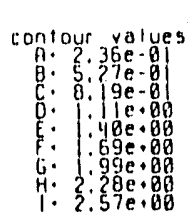

Llar impact problem

1,me. 6.00000e.01

contours of elf. plastic strain

max: 2.804e-90 in element 97 ?

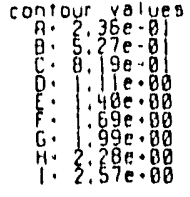

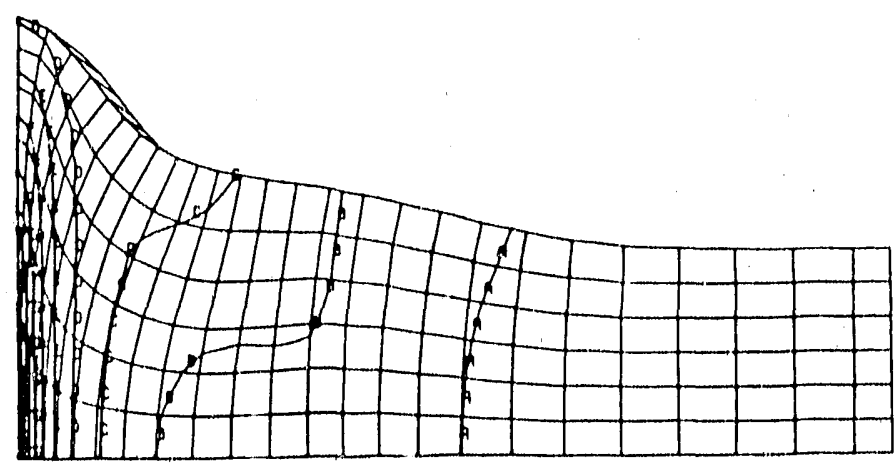

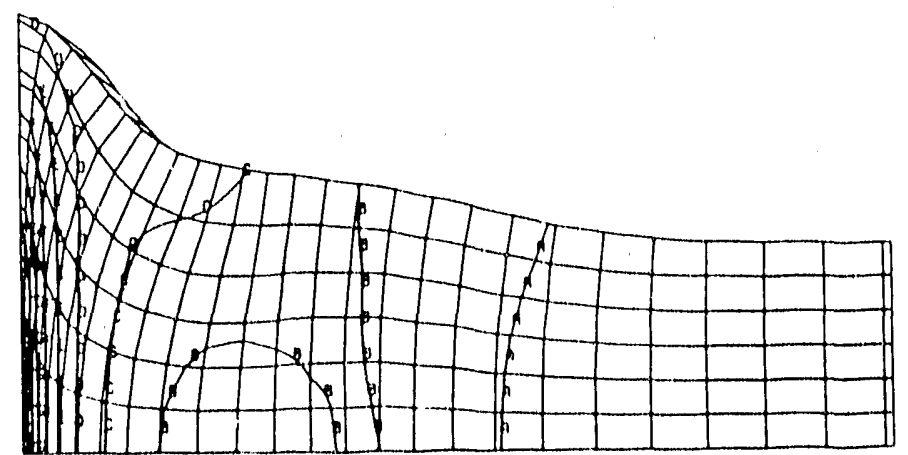


bor impoct oroblom

Plane 0.500000+01

contours of off. plastic stroin

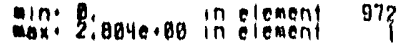

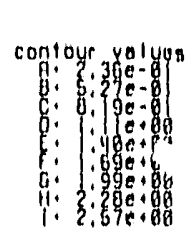

bar Inpact problen

time? 7,00009e+01

confourg of off, plastic strain

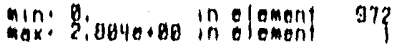

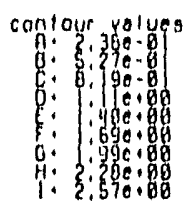

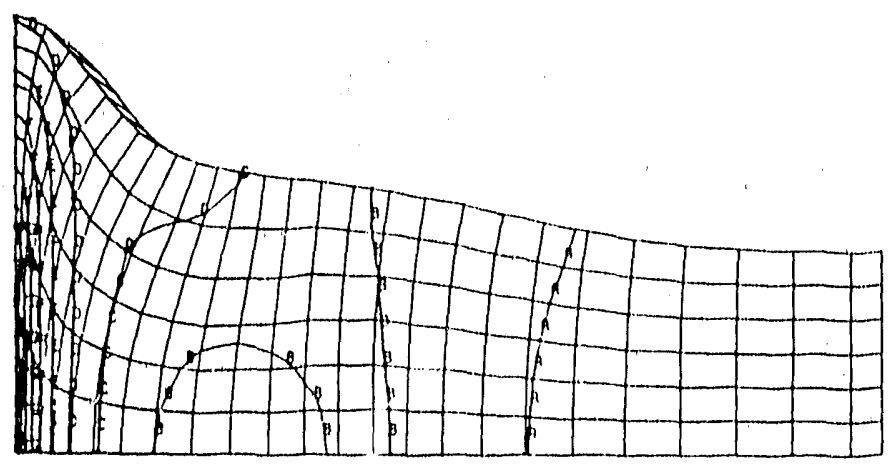

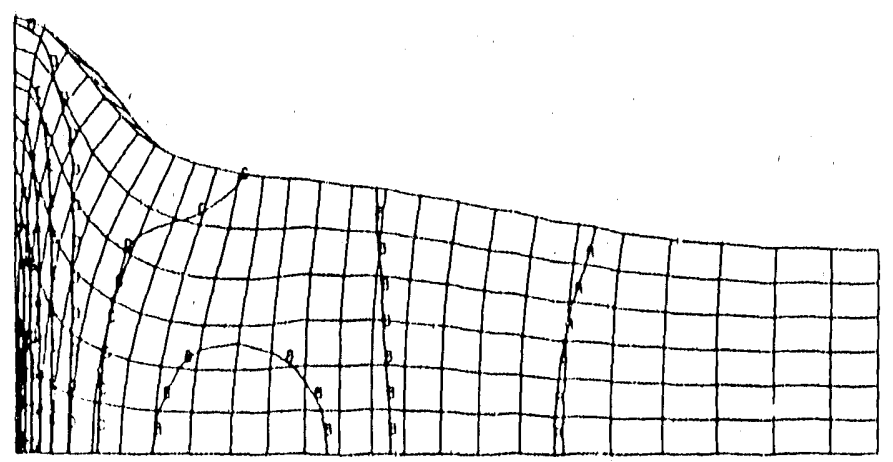

Dor impact problem

time 7.500000 .8

conpqurs of eff, plogtic stroin

mox: 2.8040.80 in element 972

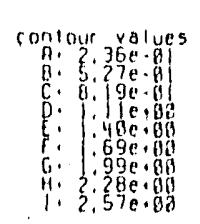

bar imact fir oolem

line . B.800606e.81

confours or eff. olastic strain

max. g.864e.80 in element 972

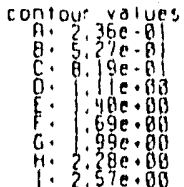

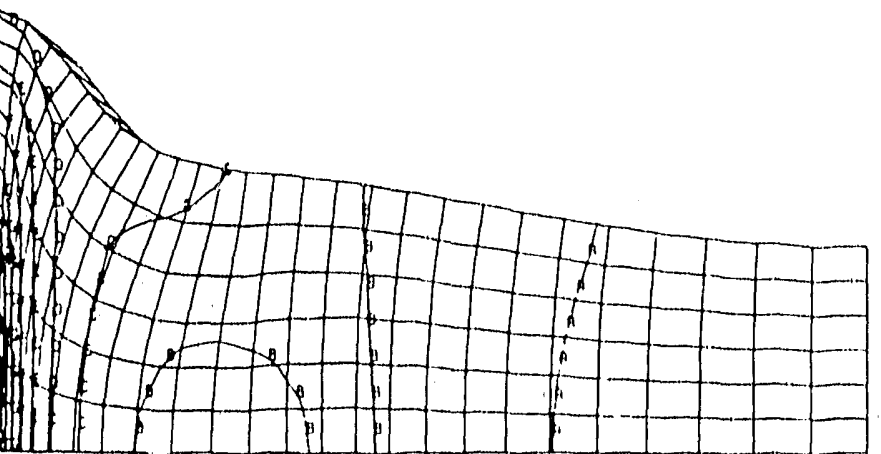

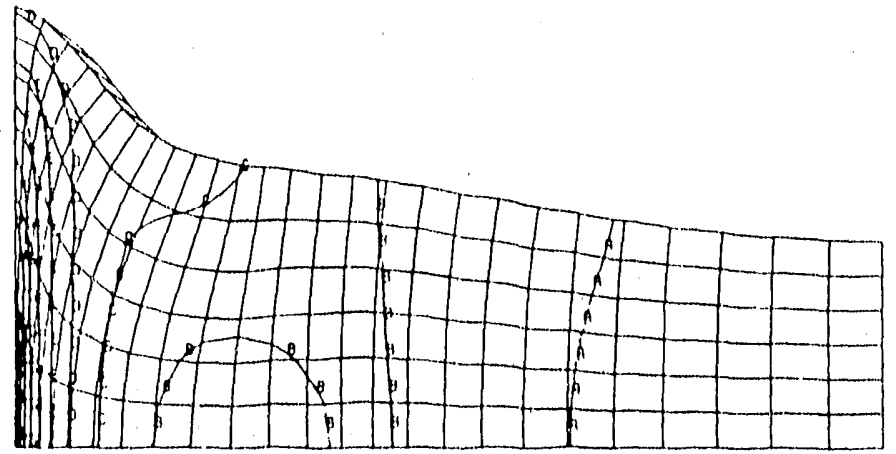




\section{INGRID/DYNA3D/TAURUS EARTH PENETRATOR EXAMPLE}

This problem is calculated with $1 / 4$ of the model using 2 planes of symmetry. The hollow projectile consists of 4 parts of steel for the casing and 1 part for the fuze and accesories. The target consists of 2 parts of concrete. All parts are generated by dragging or rotating MAZE parts from a 2 dimensional model.

Three slide surfaces are used. The first slide surface ties the fuze and accesories part to the steel casing. The third slide surface ties the 2 concrete parts together. This was needed due to the change in zoning. The second slide surface allows for the projectile to penetrate the concrete at the center of the target. The projectile is given an initial velocity of 20784 units towards the target along the axis of symmetry.

The input to INGRID is shown in the following. A picture of the resulting mesh is also included. This was done on the CRAY with the execute line

$$
\text { ingrid } \mathrm{i}=\text { erthpntr }
$$

where erthpntr was the command file for INGRID. When prompted by

$$
>
$$

the commands

$$
\text { stp } .002 \text { cont }
$$

were typed to complete the input to INGRID. For the VAX, the execute line was

\section{run ingrid}

and when prompted, then

$$
i=e r t h p n t r
$$

was typed. The rest was the same. 
The INGRID output file ingrido was then used as the input file to DYNA3D. When DYNA3D was completed, it produced a d3plot file. This was used by TAURUS. On the CRAY, the execute line was

$$
\text { taurus } g=d 3 p \text { lot }
$$

and after the initialization was complete, TAURUS prompted with a period for interactive graphics commands. This complete session is included in the following. 
$3 d$ duplicate of axisymmetric model

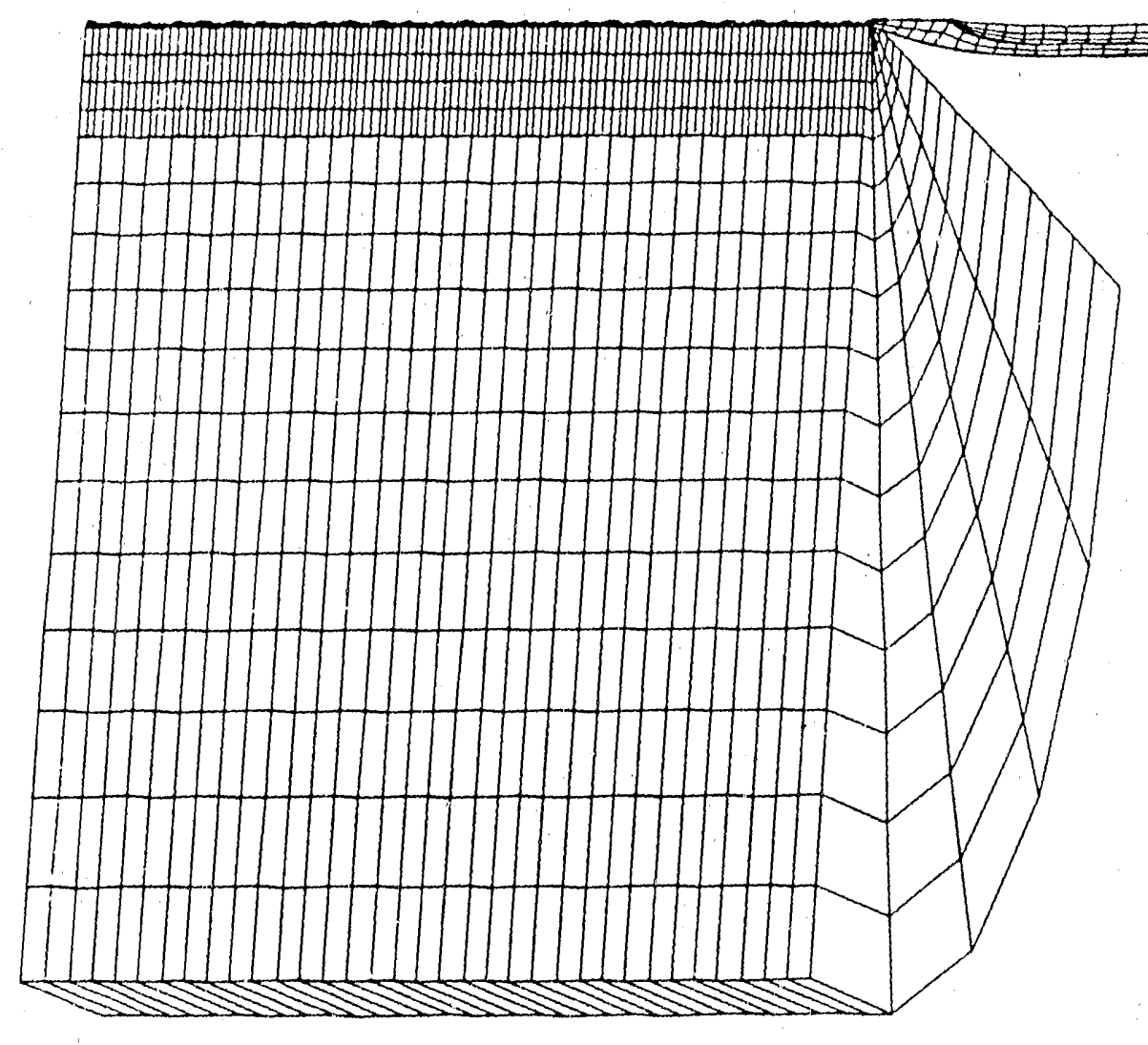


3d duplicate of axisymmetric model In $3 d$

$\begin{array}{llllllllllll}10 & 1 & 1 & 0 & 2 & .2 & 0 & .2 & 56.8\end{array}$

Id $2 \cdot 1 \mathrm{p} 2 \mathrm{D} \quad 0.7054250$

$\begin{array}{llllllllllll}\text { id } 3 & \text { ip } & 4 & 2.725 & 56.8 & 2.725 & 46.6622 & 2.475 & 32.8622 & 2.475 & 14.9425\end{array}$ lar $.7175467 .31183-17.4445$

lar $07.118885-1.4307199$

Id $4 \quad 102014.9425 \quad 3.475 \quad 14.9425$

Id 5 Ip. $23.475 \quad 56.8 \quad 3.475 \quad 14.9425$

Iap $.705425 \quad 0-38.225 \quad 14.9425$

Id 7 |pil 13 IVC -305

Id 9 IVC $0 \quad 3.00-304$.

$\begin{array}{lllllllll}1 d & 12 & 1 p & 2 & 0 & 49.53 & 3.475 & 49.53\end{array}$

Id 14 Ip $20056.8 \quad 3.475 \quad 56.8$

Id 25 ip 2.705 o 198 o

$\begin{array}{llllllll} & \text { Id } 27 & \text { lp } & 2 & .705 & -72 & 198 & -72\end{array}$

Id 22 Ip $2.705 \quad 0.705-336.75$

Id 24 Ip $2 \quad 11100111-336.75$

ld 28 ip $290 \quad 0 \quad 90-336.75$

sı 1 tied:si 2 svisi 3 tied:

plane 2000010.001 symm 00010100.00 ! symm. term 1.51 e-3 orti. . plt, $100 . e-6$

mat 13 head

steel case - 6dac

ro $7.202 e-4$

e $3 . e 7$

pr. . 3

sigy $194 . e 3$

etan 1.De5

mat 23 head

fuze and accesories

ro $2.065 e-4$

e $3 . e 7$ pr .3 sigy 174. e 3 etan $\emptyset$

mat 35 head

concrete

ro $2.1692 e-4$

g. $788 \mathrm{e} 6$

ku $.600 \mathrm{e} 7$

$\mathrm{ab} \cdot 352 \mathrm{e} 7$

al 8570

a2 0

DC 0

npts 6

vs $0.020 .0377 \quad .0418 .0513 .1$

P 021000348004500058000 i25000

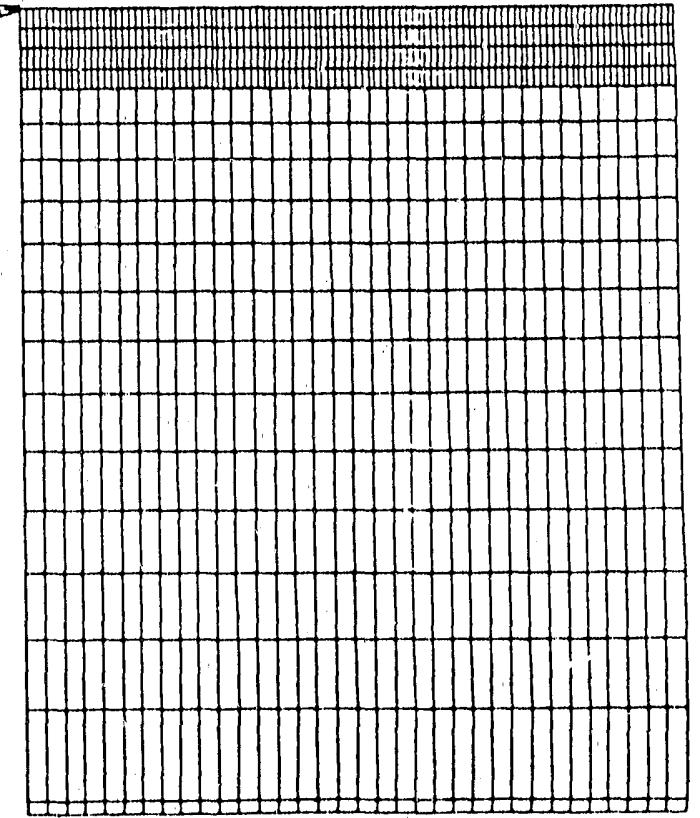
eñứàt 
part 25091122

drag rota 5000000190 :

si $2112222 \mathrm{~m}$

velo $00-20784$.

end

part 957.1123

drag rota 5000000190 :

si $2112222 \mathrm{~m}$

velo $00-20784$.

end

part 7543125

drag rota 5000000.190 :

si $2112222 \mathrm{~m}$

velo $00-20784$.

end

part $4514 \quad 3 \quad 31220$

drag rota 5000000190

si $21112222 \mathrm{~m}$

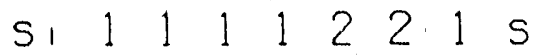

velo $00-20784$.

end

part $123 \quad 3 \quad 14 \quad 1.223$

drag rota 500000190 :

si $2112221 \mathrm{~m}$

velo $00-20784$.

end

part $22 \quad 2724 \quad 25 \quad 3 \quad 1024$

drag rota 5000000100 :

s. $1 \begin{array}{lllllll}1 & 1 & 2 & 2 & 1 & 2 & 5\end{array}$

si 11222235

end

part $\begin{array}{llllllll}28 & 25 & 24 & 27 & 3 & 34 & -12 & 2.0\end{array}$

drag rota 50000000100

si $1122223 \mathrm{~m}$

end

enid

c use the command stp.obl 
The following is the interactive input to TAURUS after this program has initialized. The period is the prompt from TAURUS.

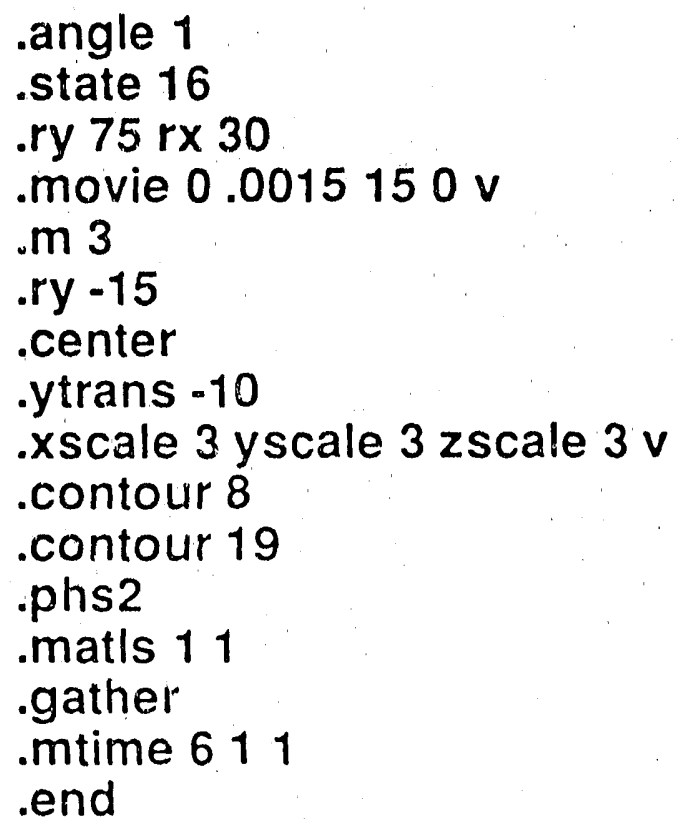


30 duplicote of axisymmetric model

rime - 8.

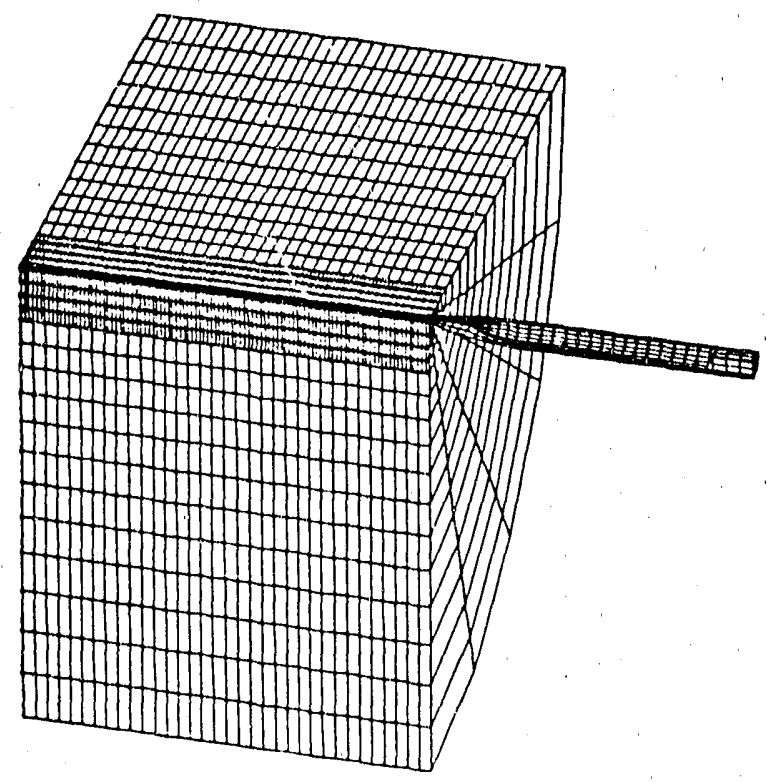

3o duplicate of axisymmetric model

time $2.00800 \mathrm{e}-04$

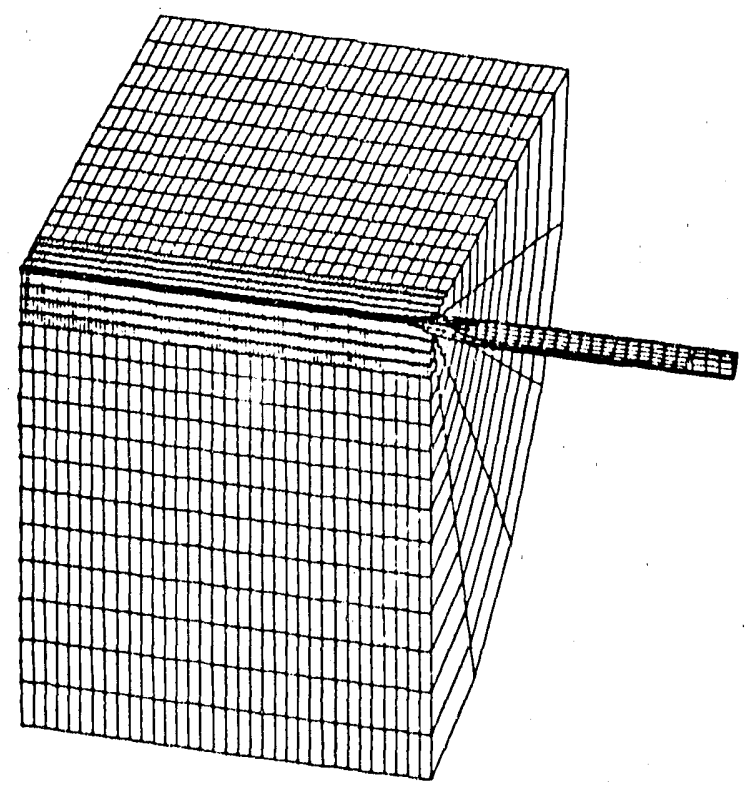

$3 d$ duplicate of axisymmetric model

time $1.90000 \mathrm{e}-84$

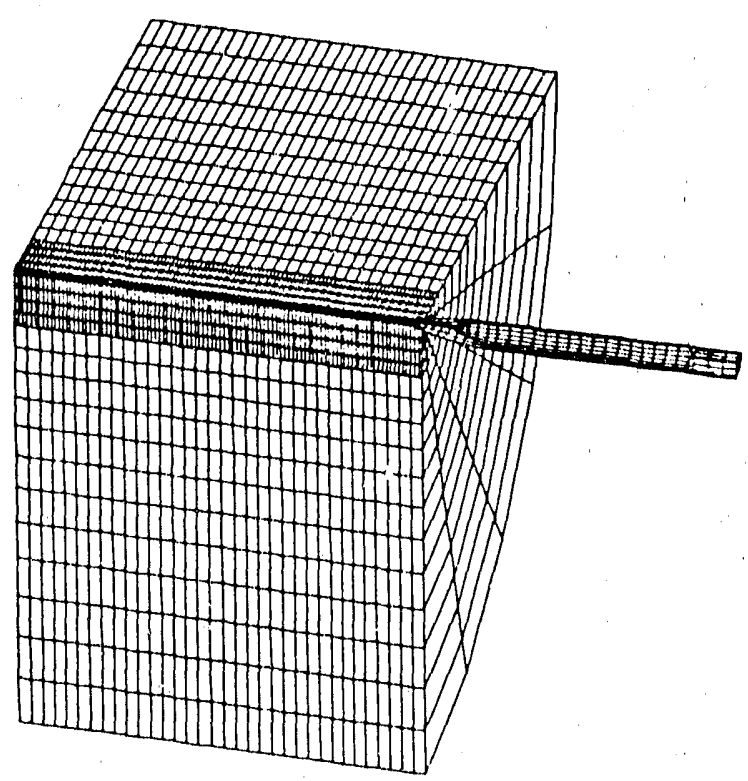

3 duplicate of axisymmetr ic model

time $3.00000 \mathrm{e}-04$

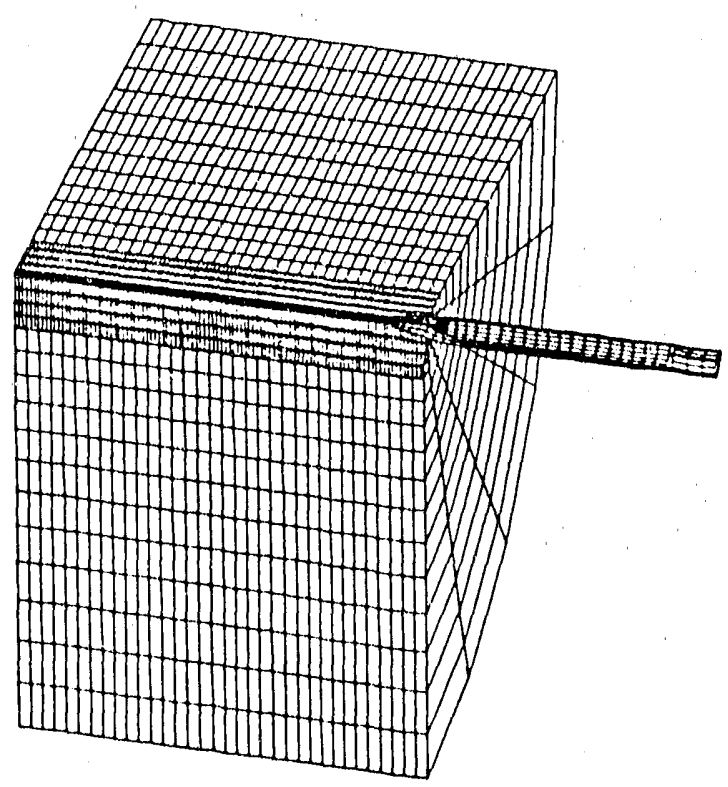


30 duplicate of axisymmetric model

lime: $4.00000 \mathrm{e}-04$

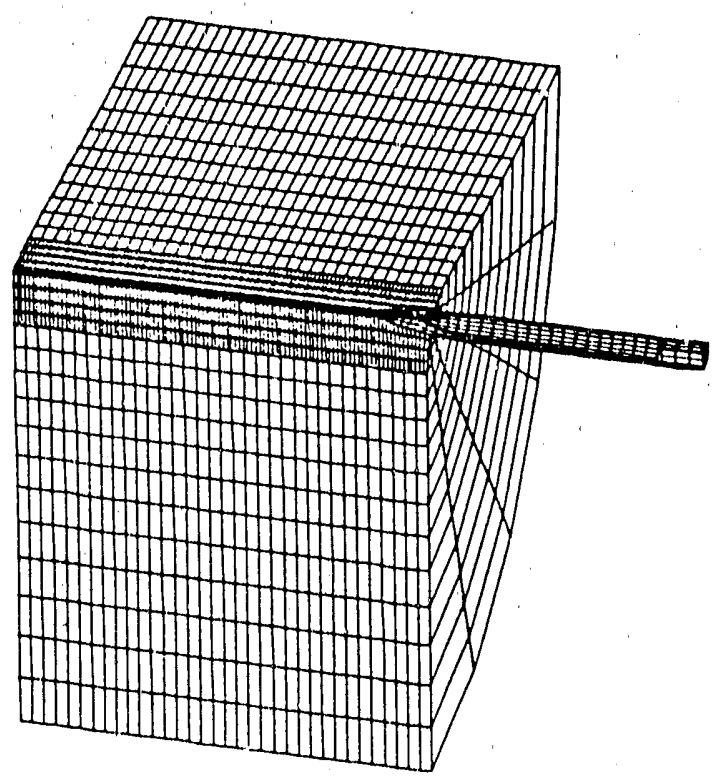

30 duplicate of axisymmetric model

time. $6.00000 \mathrm{e}-84$

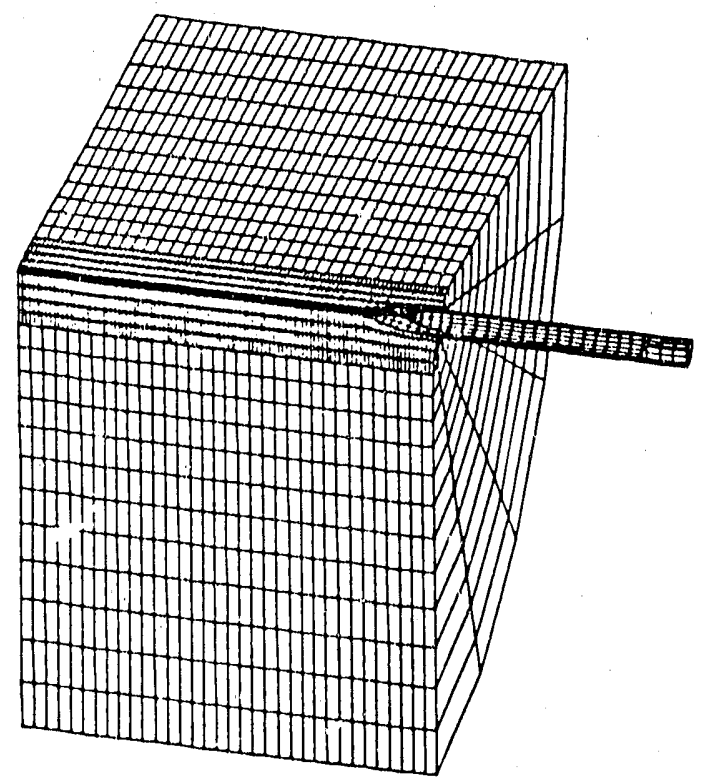

3d duplicate of axisymmetric model

time. $5.000000-04$

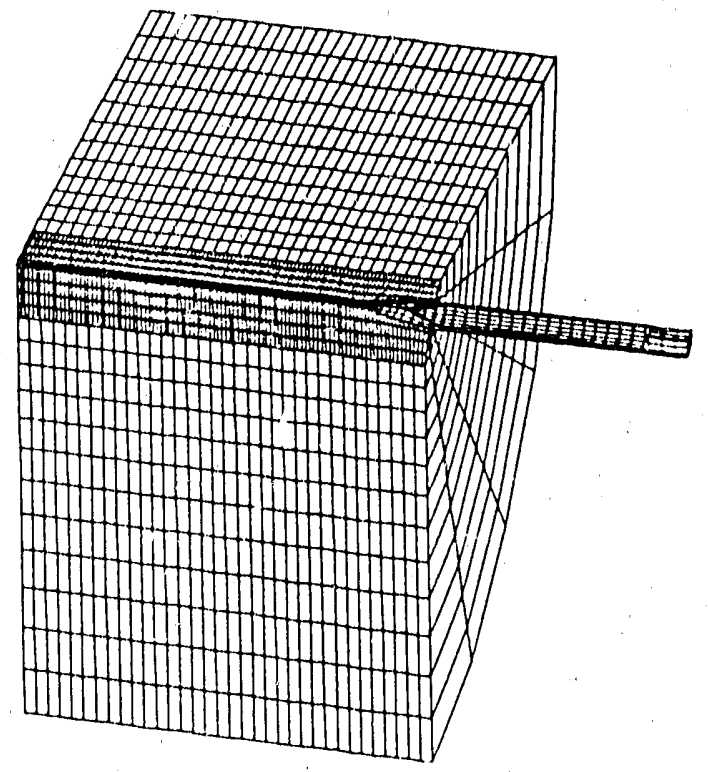

30 duplicale of ax, symmetr ic model

time. $7.00000 \mathrm{e}-84$

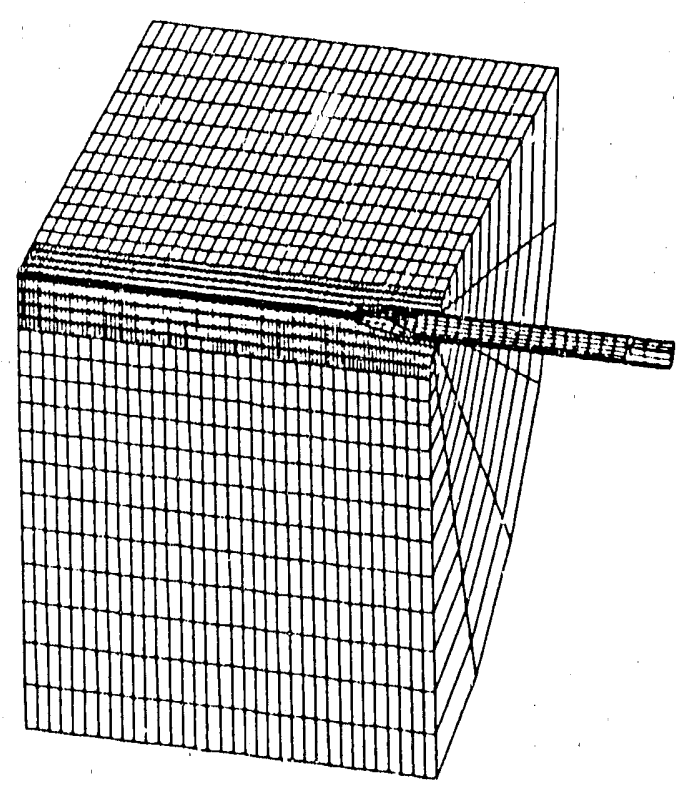


3d duplicote of axisymmetr ic model

time - $8.000000-84$

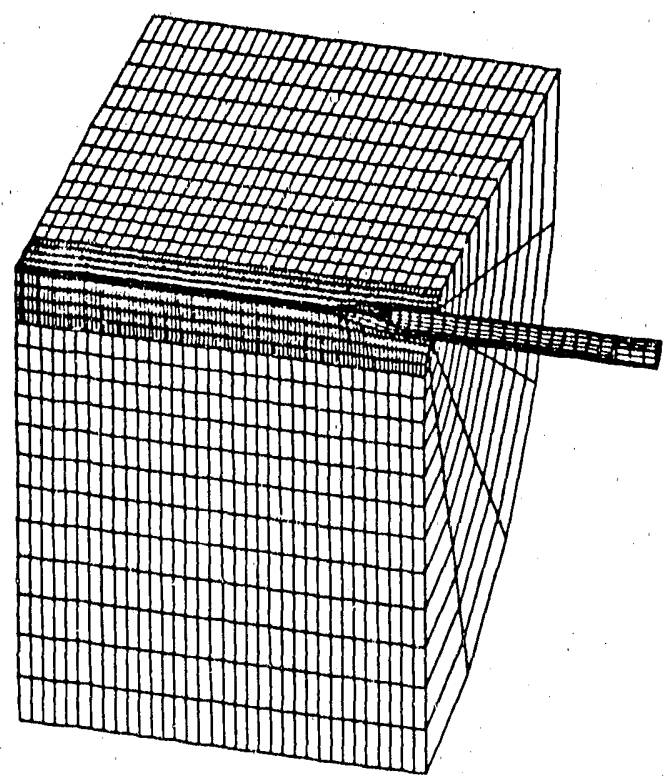

30 duplicate of axisymmetric mode

time $1.00008 \mathrm{e}-83$

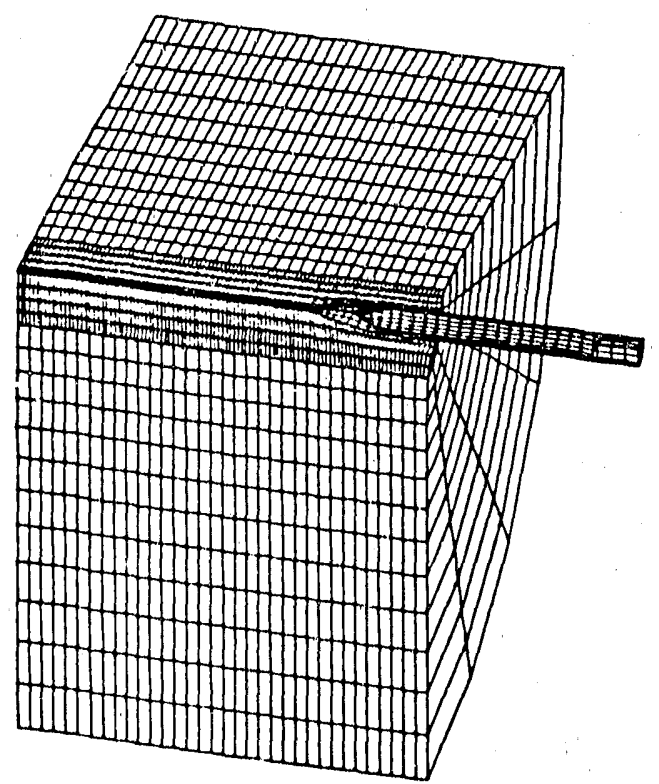

3d duplicaie of axisymmetric model

time 9.00000 e-04

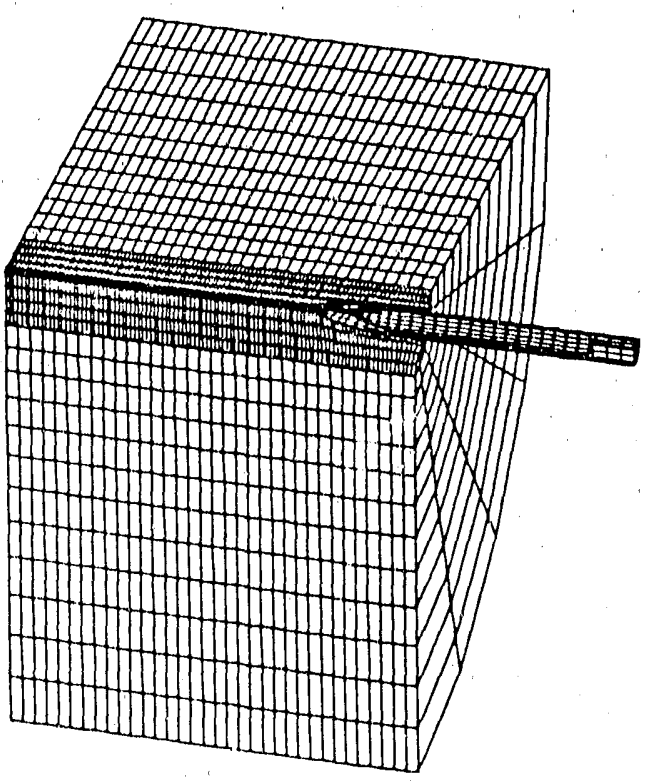

3d duplicate of axisymmetric model

lime. $1.10000 \mathrm{e}-03$

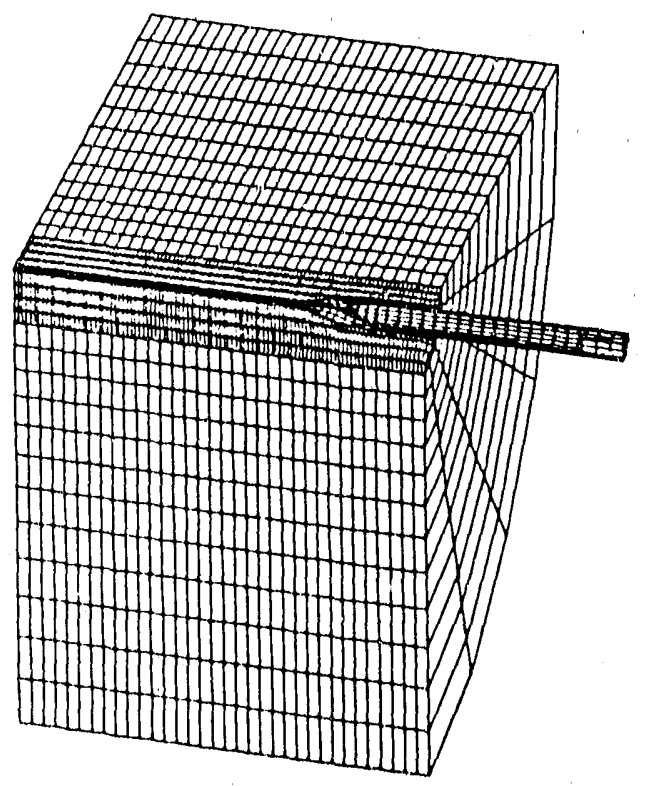


30 duplicote of exisymmetric model

time. $1.20000 \mathrm{e}-83$

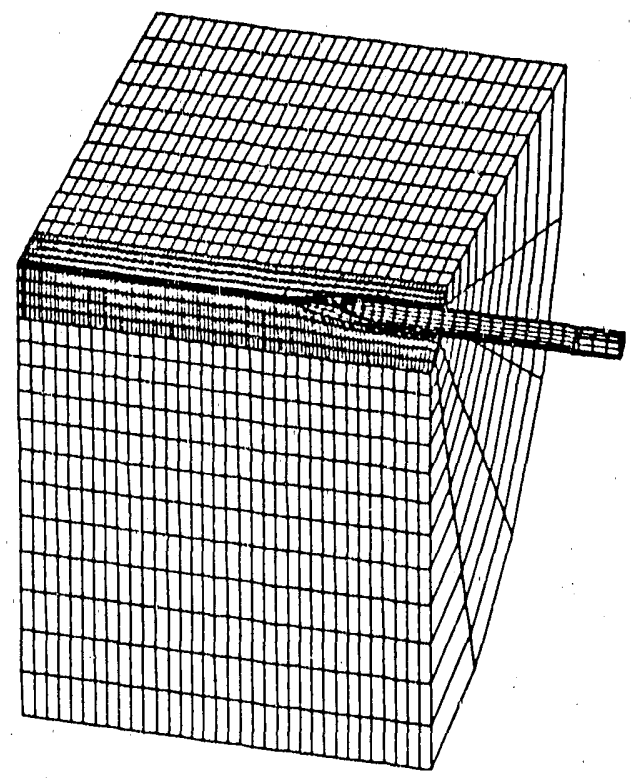

3a duplicate of axisymmetric model

time $1.40000 \mathrm{e}-03$

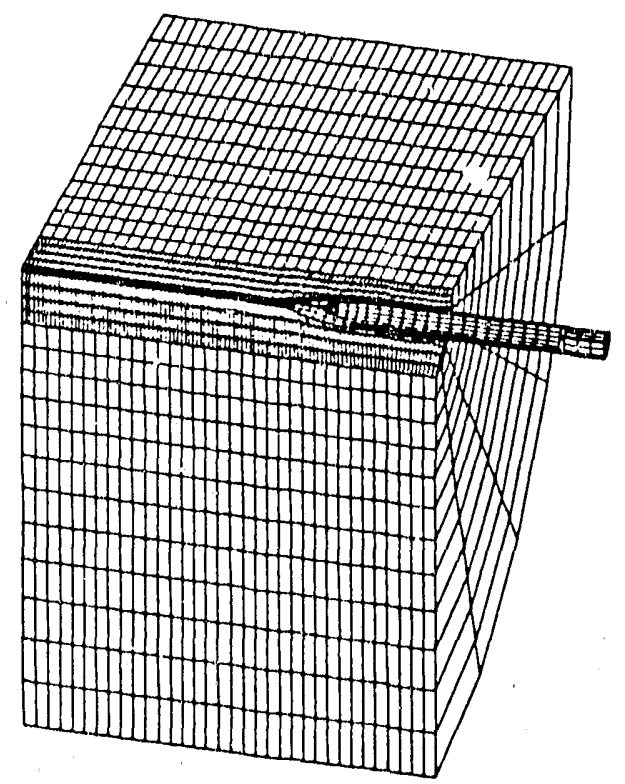

3d duplicate of axisymmetric model

time $1.30000 \mathrm{e}-03$

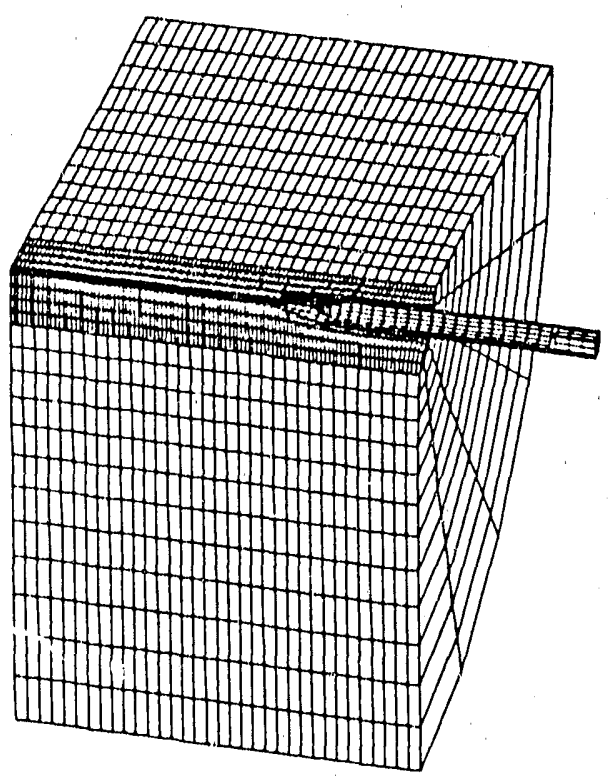

3o duplicate of axisymmetric model

1.me. 1.50000 e-03

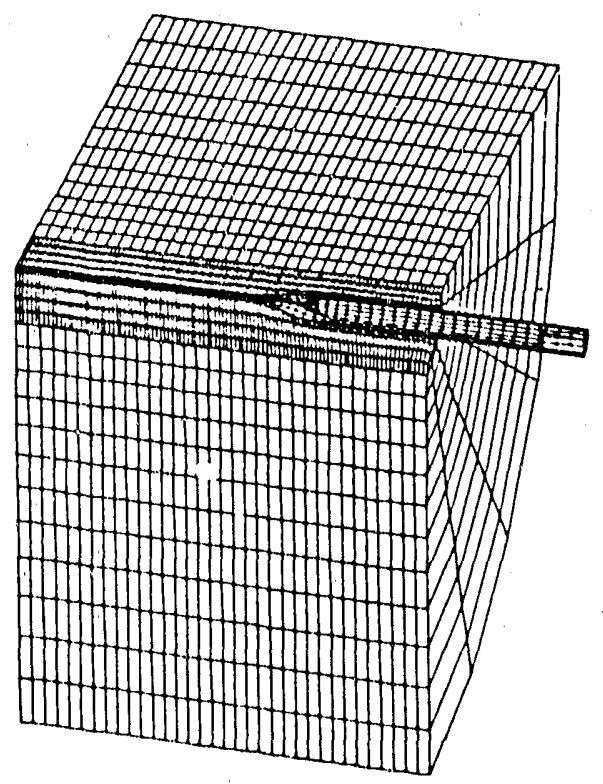




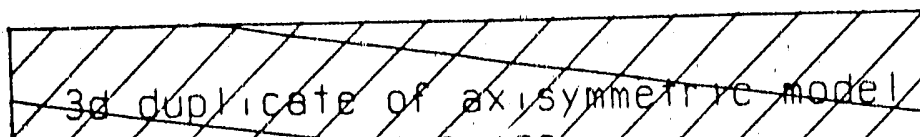
time 7. . 99096003

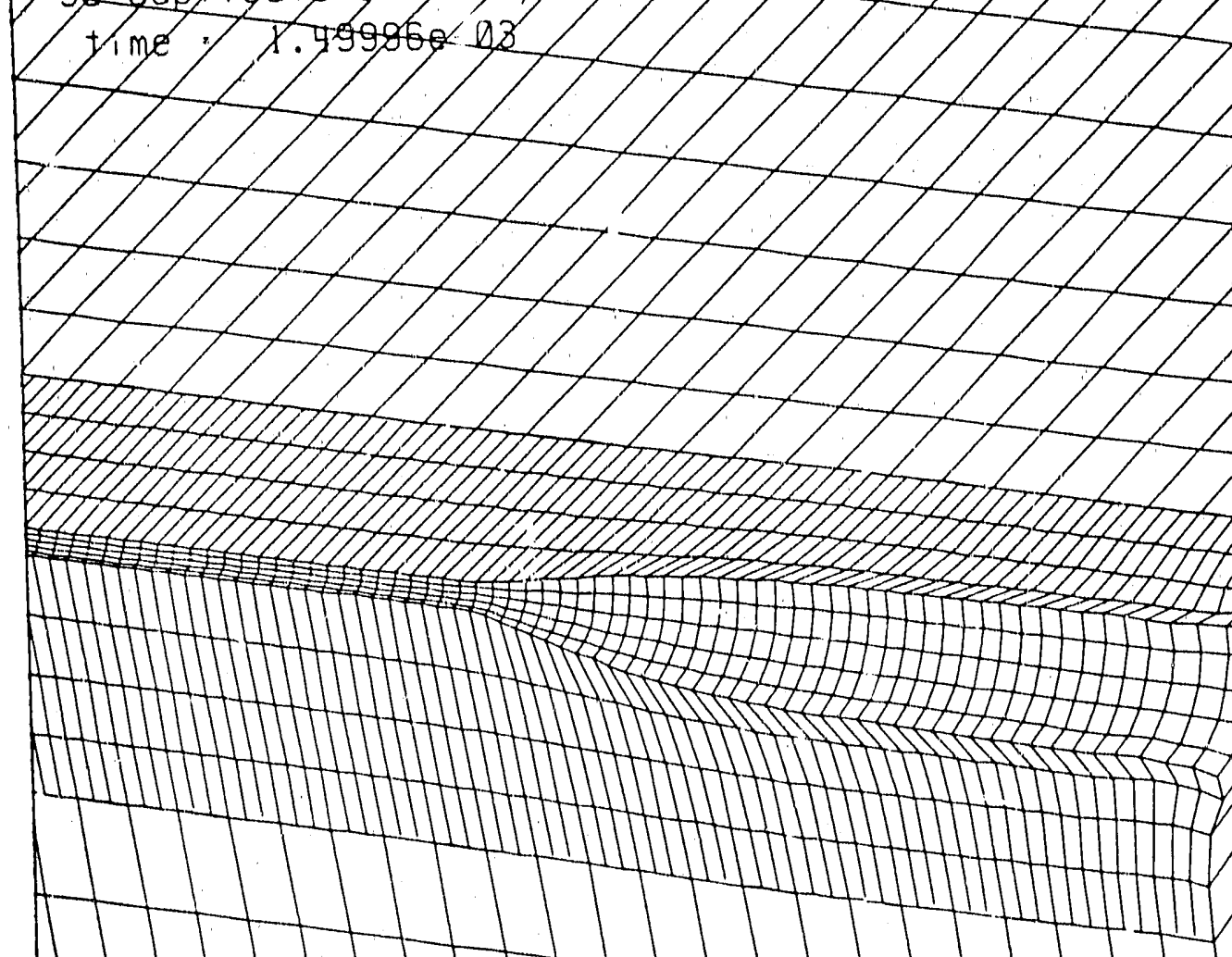




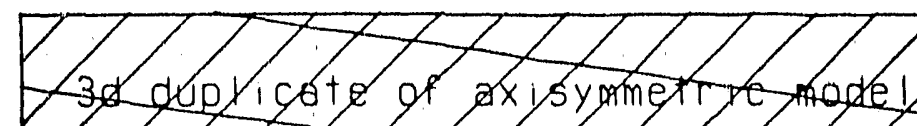
Jime $=7.099960-03$

tcontours of pressurire

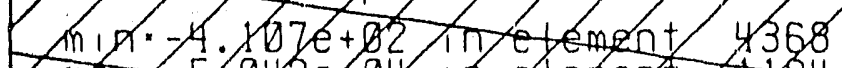

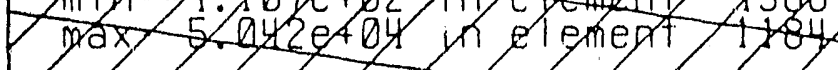

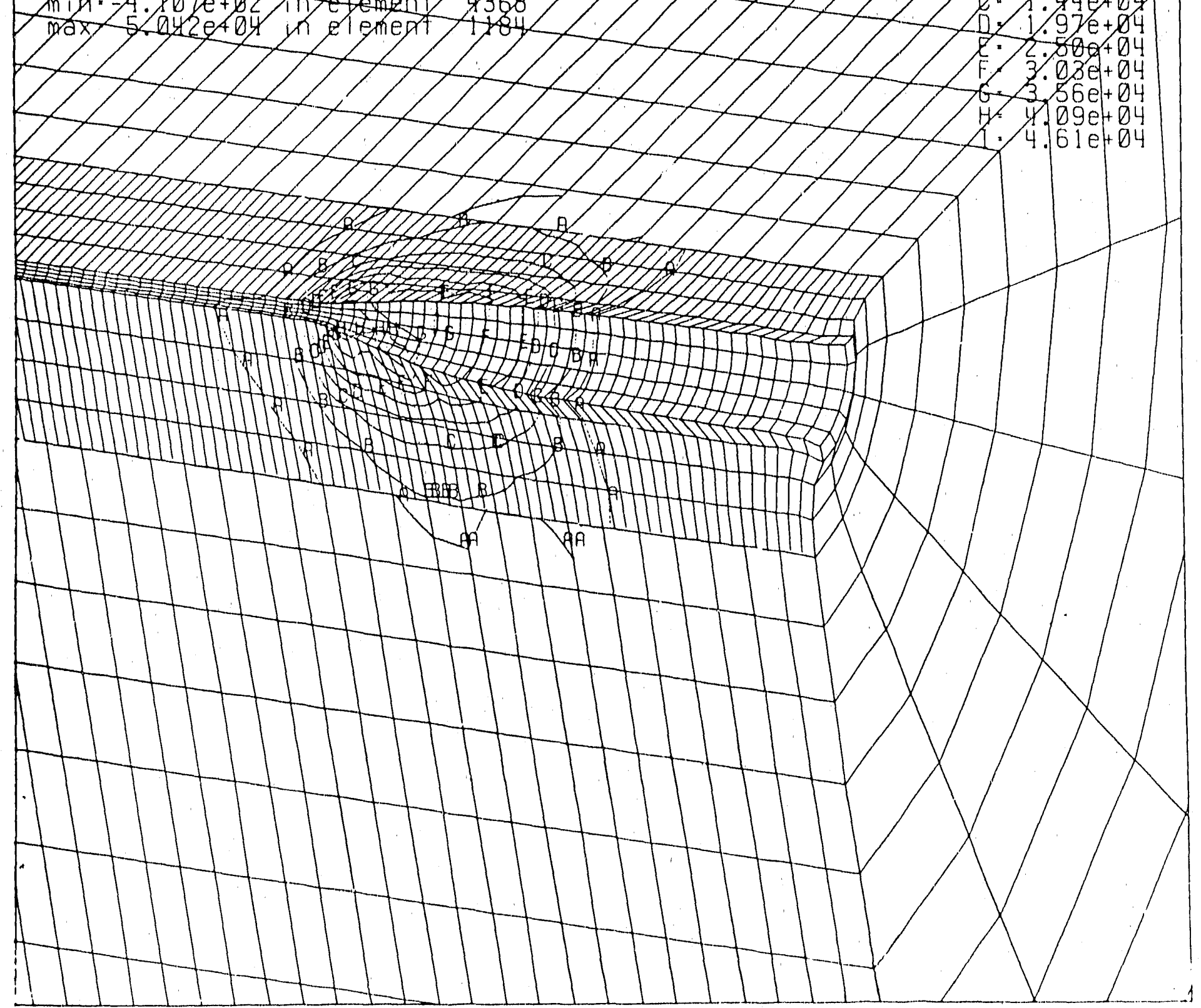




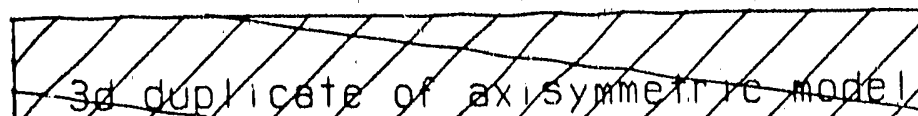

time $7.099960-03$

Conntours of z-di splacement

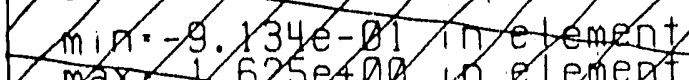

max i 625 e 400 in elemedt 


\section{$109 / 110$}

3d duplicate of axisymmetric model

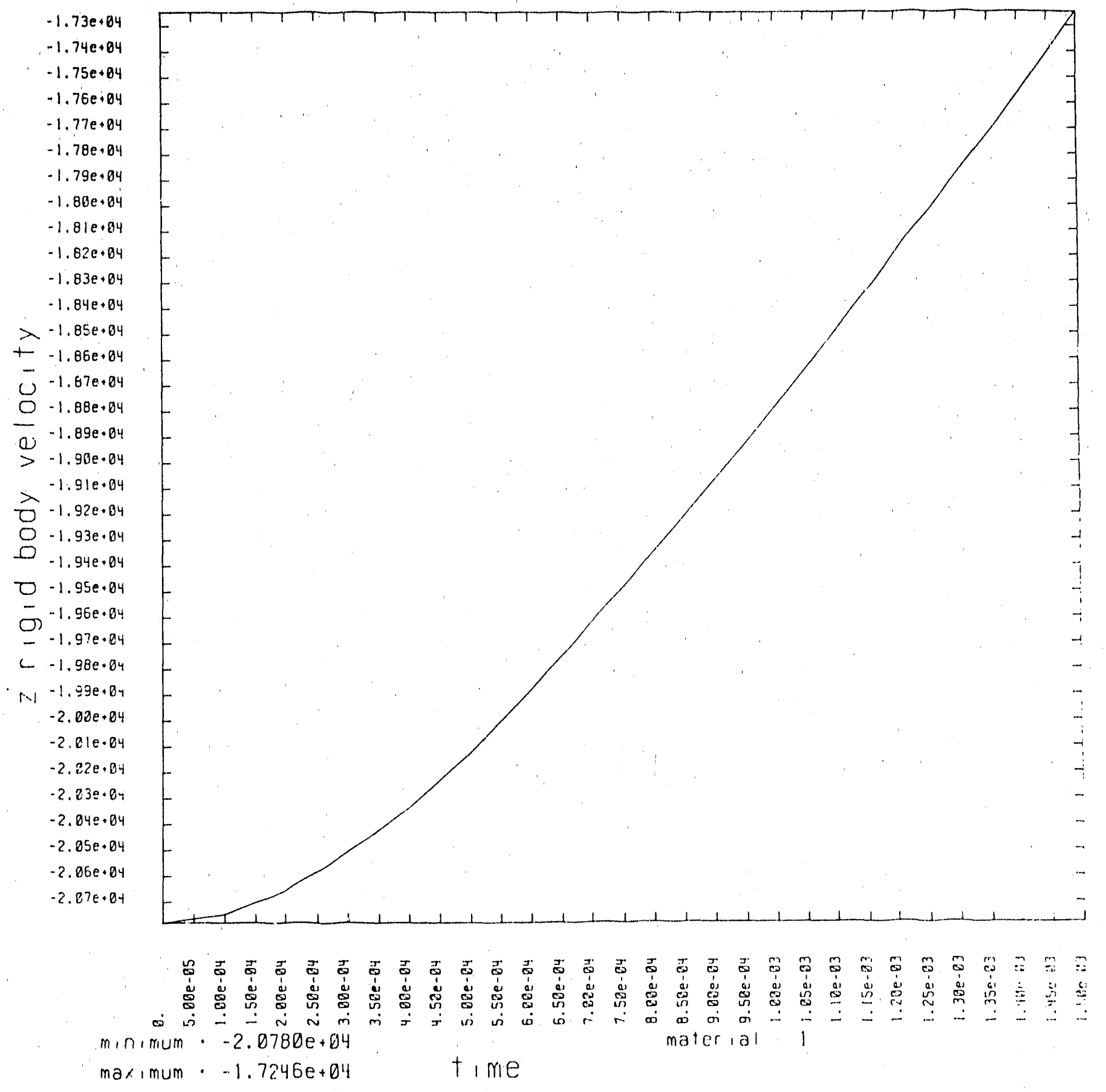




\section{INGRID/NIKE3D/TAURUS PIPE WHIP EXAMPLE}

The problem is calculated with $1 / 2$ of the model using 1 plane of symmetry. Half of a pipe is modeled to impact a second pipe. Both pipes are modeled in INGRID with thin shells which are four node shell elements in NIKE3D. Both pipes are doformed. NIKE3D is stopped before the pipes spring back and come to rest.

When INGRID is in the interactive mode, use the command stp .0001 continue

to connect the two edges of the second pipe and write the input for NIKE3D. 
pipe whip by r. ferencz.

nk3d anal dyn

Dwmo off

nosr 1 nbei 1 nibsr 10 msrf 15

actol. .001 ectol.01

iprt 99 iplt 1 nstep 200 term $10 \mathrm{e}-3$

mat 13 shell ro 7.298 e-4 e 3. De+7

pr.3 sigy $1.0 \mathrm{e}+5$ etan $1.0 \mathrm{e}+5$

mat 23 shell ro $7.298 \mathrm{e}-4$

e $3.0 \mathrm{e}+7 \mathrm{pr} .3$ sigy $1.0 \mathrm{e}+5$

etan 1.0 e 5 endmat

plan 10000100.001 symm

coor $2 r+000 r+100$ sp $190-16$

$r+0025 r+0026 r+0125$

start - - : : 1 8 15:1 $10 \quad 24 \quad 33$ :

$3.3125 \quad 90 \quad 180 \quad 270 \quad 0 \quad 18 \quad 3250$

cyli thic 432

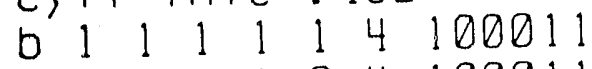

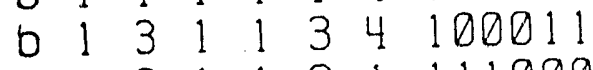

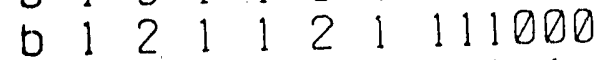

$51-122 \begin{array}{lllllllll}1 & 1 & 3 & 3 & 1 & 1 & 0 & 0 & 0\end{array}$

rota 1007500

repe 1 : end

start - 1:1 29:1 7 15:

$3.31250360 \quad 0725$

cyli thic .432

$n-111122200.12 .5$

b 0001300030111111

b

si- 111111222100025

mate 2 repe 2 : end end

c use stp in iteractive phase

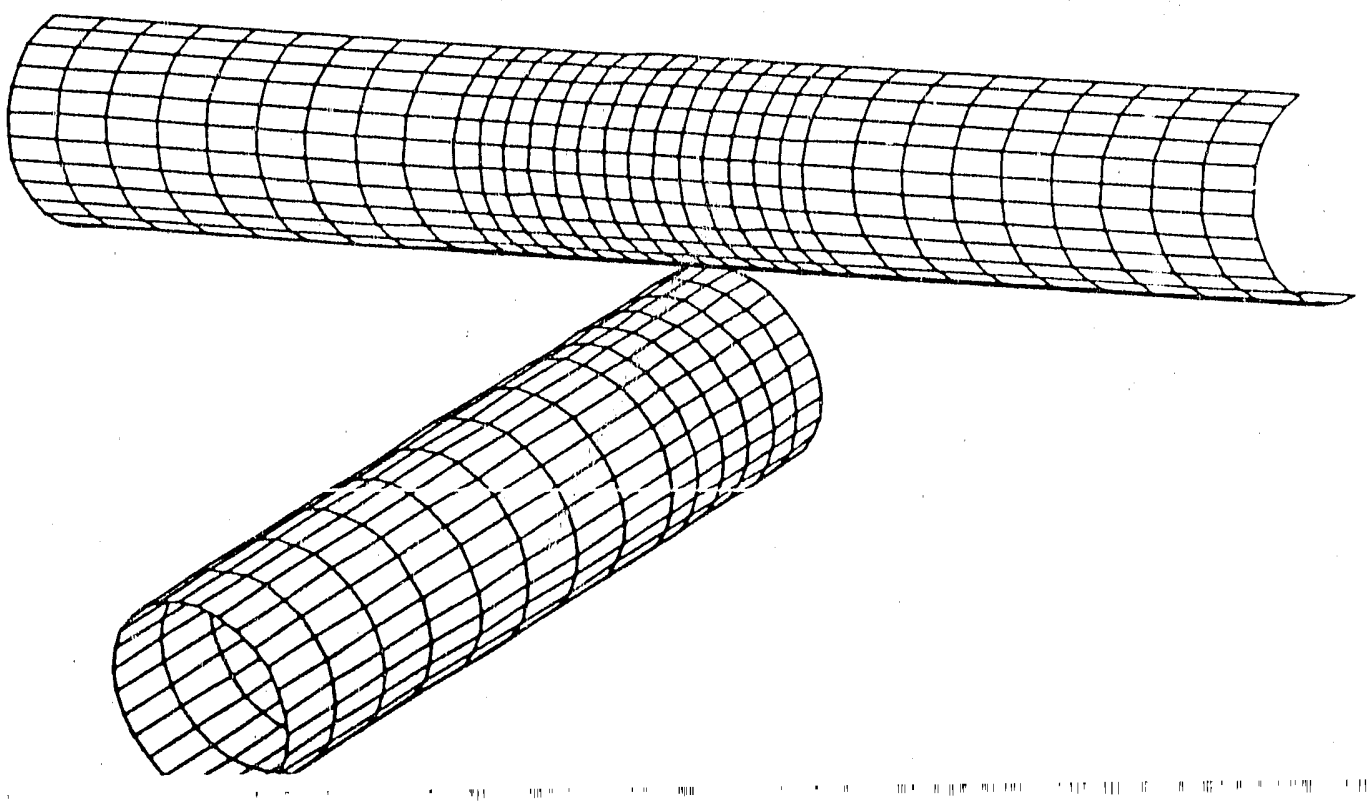


The following is the interactive input to TAURUS after TAURUS was initialized with the plot files from the NIKE3D run. The period is the prompt from TAURU: ror more input.

.angle 20

.ry -50

$.1 \times 20$

.xscale 4 yscale 4 zscale 4

-ytrans 7

.xtrans -2

movie $0.01150 \mathrm{v}$

.end 

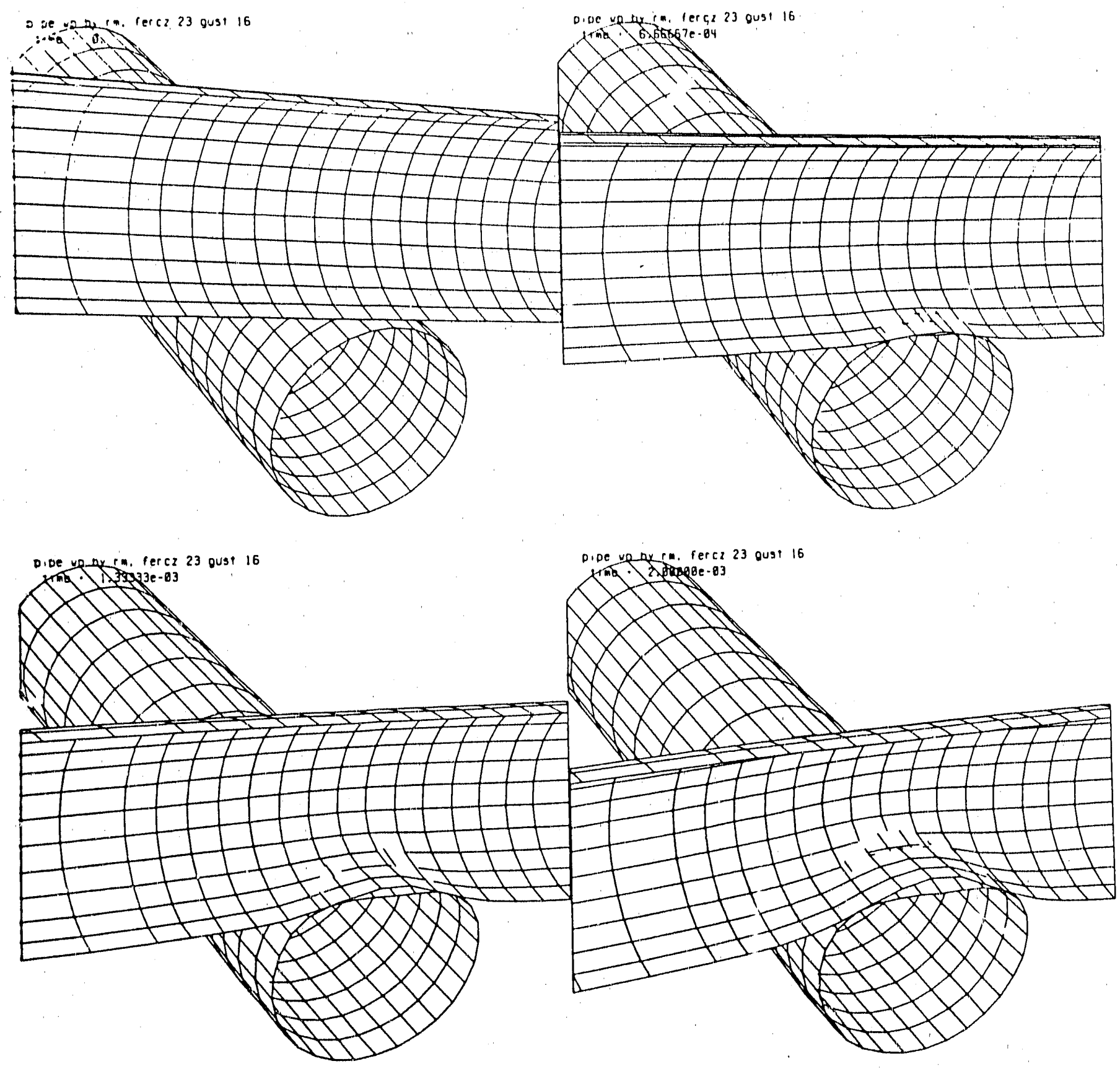

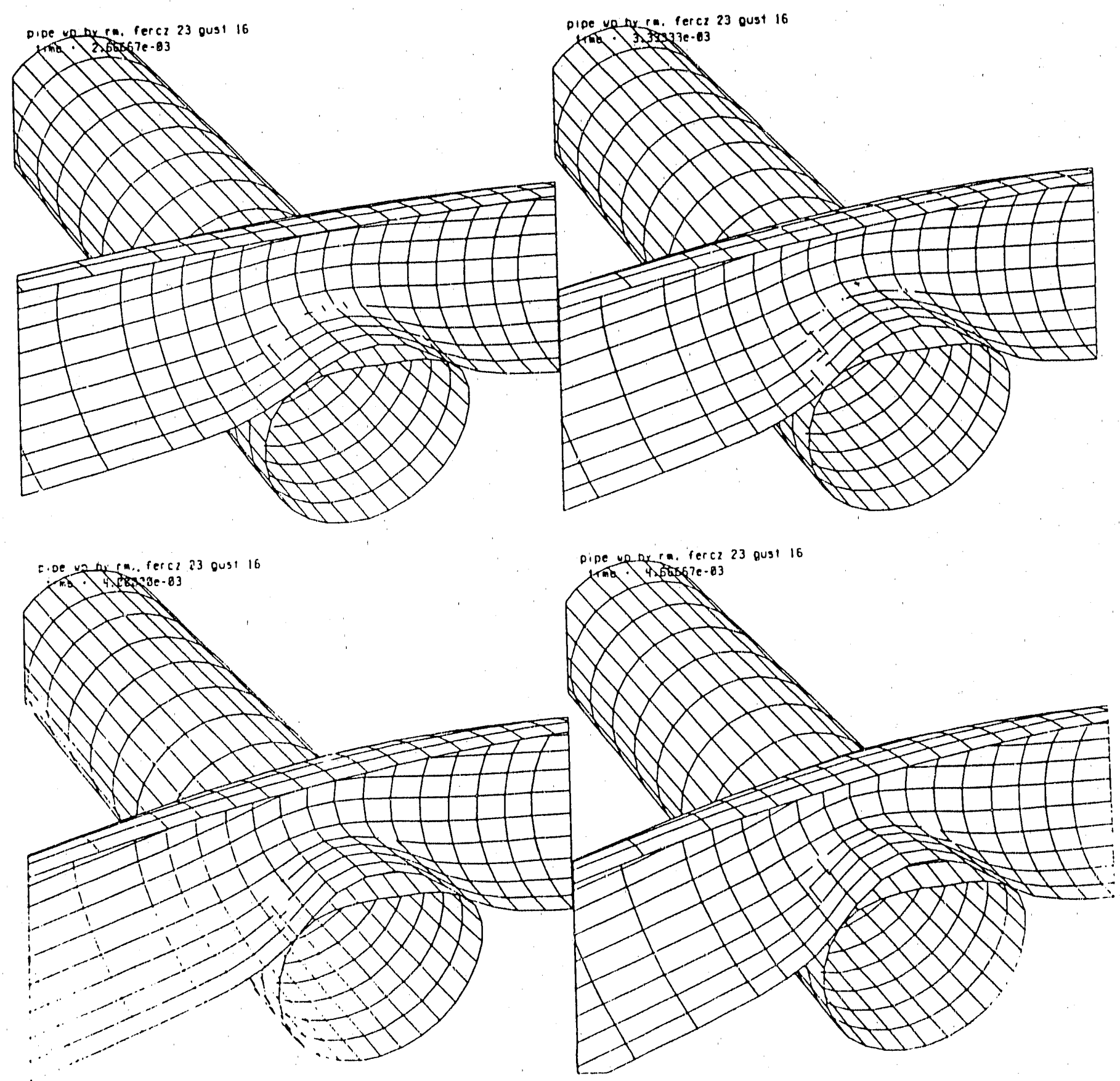

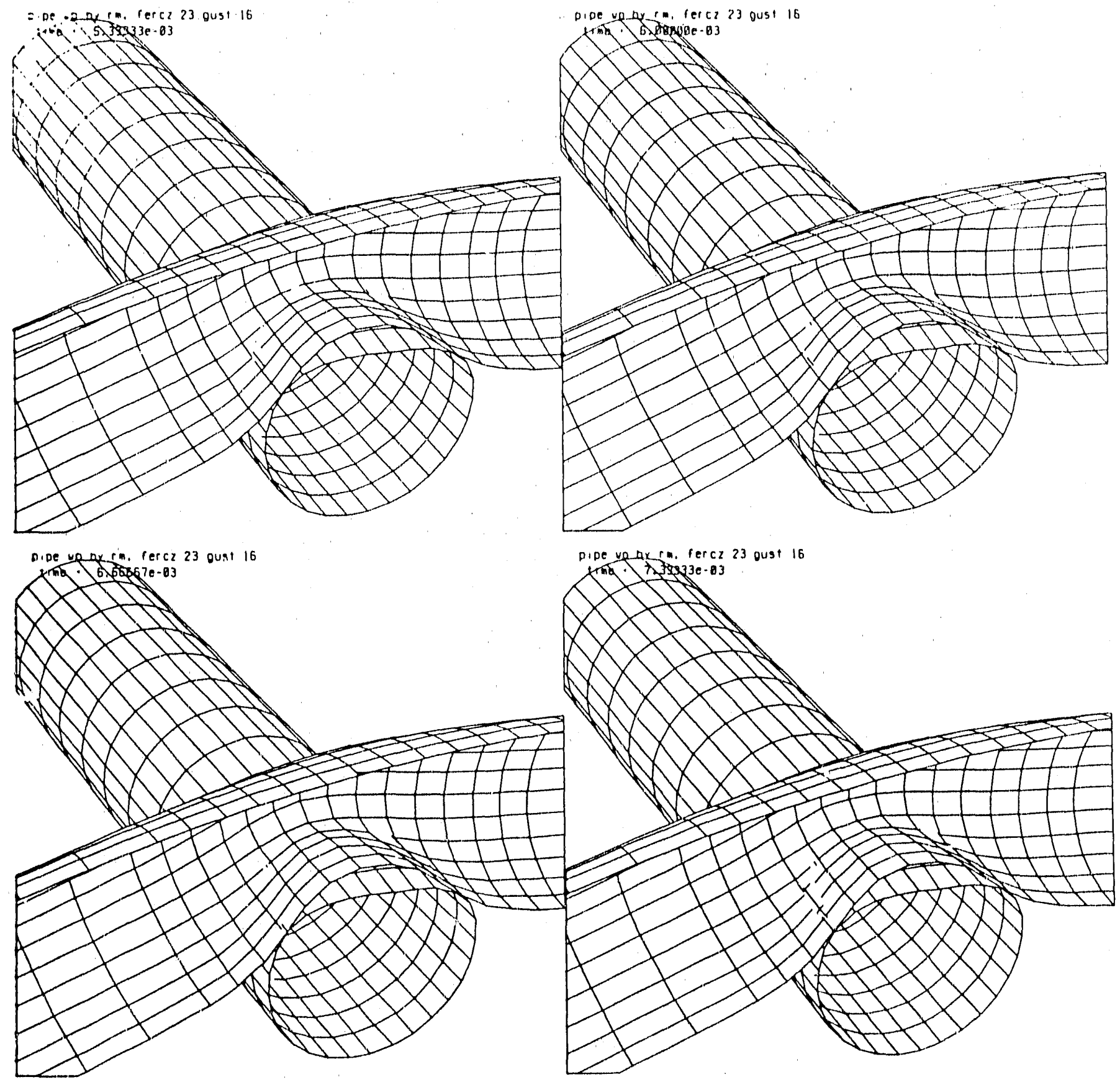

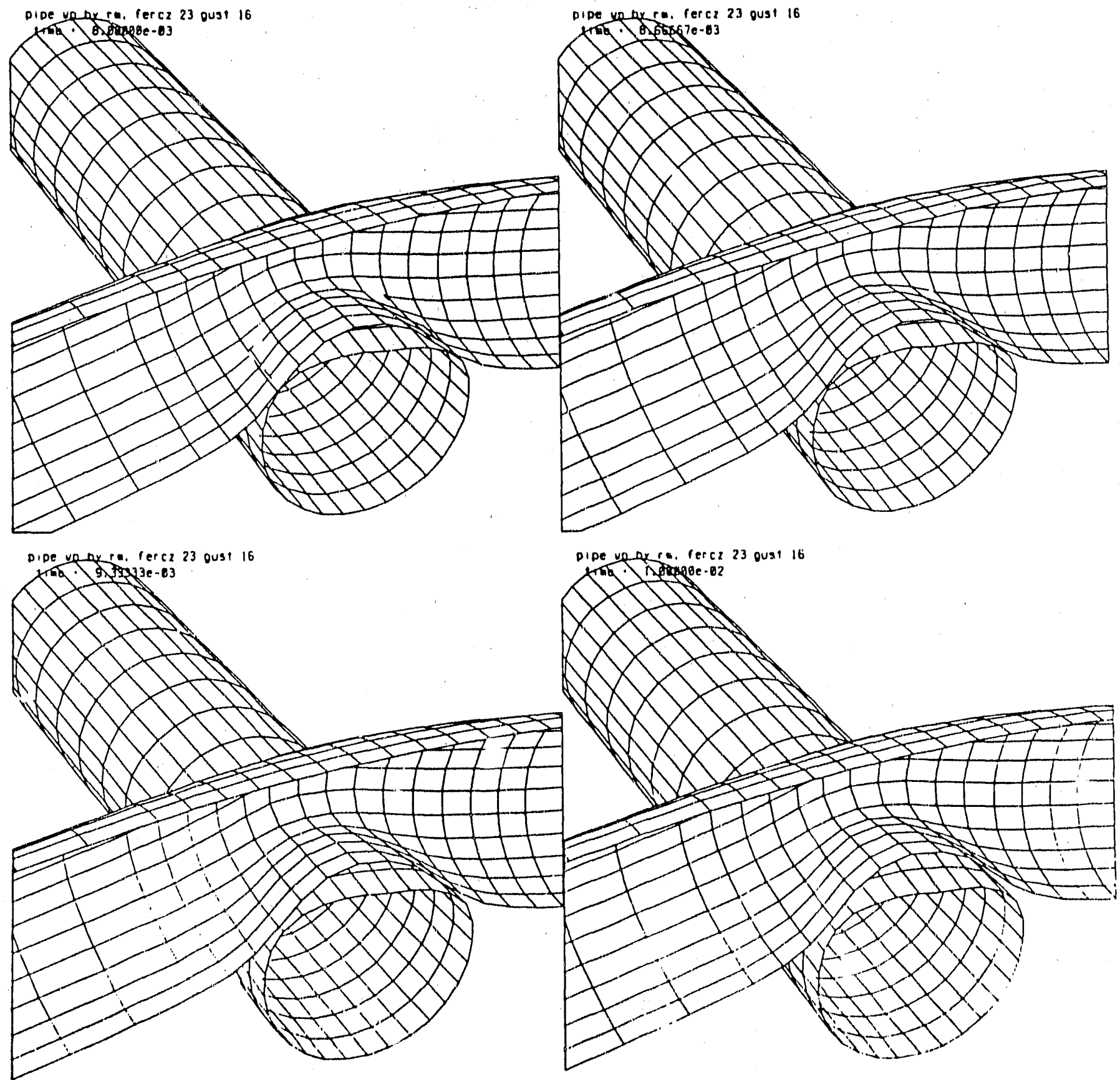

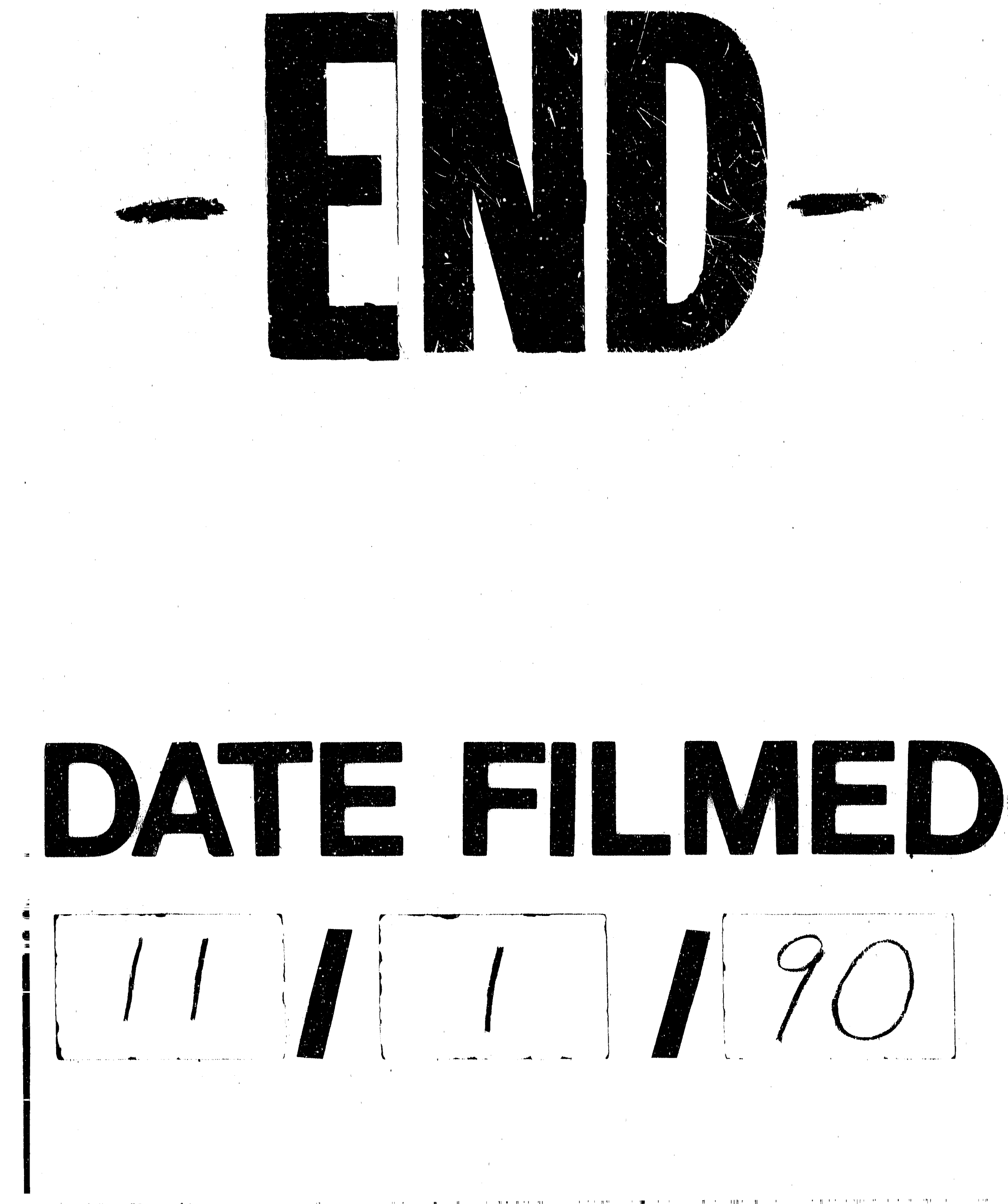
